\title{
CHANGES IN EXERCISE CARDIAC FUNCTION WITH AGE IN ENDURANCE TRAINED FEMALES
}

\author{
COLLEEN WIEBE
}

\begin{abstract}
A Thesis submitted to the faculty of Graduate Studies in partial fulfilment of the requirements for the degree of

Master of Science
\end{abstract}

Graduate Programme in Exercise and Health Science

York University

North York, Ontario

January 1998 
National Library

of Canada

Acquisitions and

Bibliographic Services

395 Wellington Street

Ottawa ON K1A ON4

Canada
Bibliothèque nationale

du Canada

Acquisitions et

services bibliographiques

395, rue Wellington

Ottawa ON KIA ON4

Canada
The author has granted a nonexclusive licence allowing the National Library of Canada to reproduce, loan, distribute or sell copies of this thesis in microform, paper or electronic formats.

The author retains ownership of the copyright in this thesis. Neither the thesis nor substantial extracts from it may be printed or otherwise reproduced without the author's permission.
L'auteur a accordé une licence non exclusive permettant à la Bibliothèque nationale du Canada de reproduire, prêter, distribuer ou vendre des copies de cette thèse sous la forme de microfiche/film, de reproduction sur papier ou sur format électronique.

L'auteur conserve la propriété du droit d'auteur qui protège cette thèse. $\mathrm{Ni}$ la thèse ni des extraits substantiels de celle-ci ne doivent être imprimés ou autrement reproduits sans son autorisation. 
CHANGES IN EXERCISE CARDIAC FUNCTION

WITH AGE IN ENDURANCE-TRAINED FEMALES

by

$$
\text { COLILEN WIEBE }
$$

a thesis submitted to the Faculty of Graduate Studies of York University in partial fulfiliment of the requirements for the degree of

\section{MASTER OF SCIENCE}

c)

$$
1998
$$

Permission has been granted to the LIBRARY OF YORK UNIVERSITY to lend or sell copies of this thesis, to the NATIONAL LIBRARY OF CANADA to microfilm this thesis and to lend or sell copies of the film. and to UNIVERSITY MICROFILMS to publish an abstract of this thesis. The author reserves other publication rights, and neither the thesis nor extensive extracts from it may be printed or otherwise reproduced without the author's written permission. 


\section{ABSTRACT}

It has been shown in recent investigations from our laboratory that while the stroke volume (SV) of normally active (NA) males reached a plateau at moderate exercise intensities ( $120 \mathrm{bpm})$, the SV of endurance trained (ET) males increased progressively up to maximum hear rate (HRmax). The progressive increase in SV to maximum in ET males was due to enhancements in both the diastolic filling rate (DFR) and left ventricular emptying rate (LVER), with the primary advantage being in the DFR. The enhanced DFR in ET compared to NA males was linked to their augmented blood volume (BV). We therefore hypothesized that similar cardiac function responses would be observed in ET females. We also hypothesized that these cardiac function responses would be maintained with age in ET females. Therefore, exercise cardiac function was examined in 23 ET females, six age 20-29 yr $\left(\bar{x} V_{2} \max =64 \mathrm{ml} \cdot \mathrm{kg}^{-1} \cdot \min ^{-1}\right)$, six age $40-45 \mathrm{yr}\left(\overline{\mathrm{x}} \mathrm{VO}, \max =51 \mathrm{ml} \cdot \mathrm{kg}^{-1} \cdot \mathrm{min}^{-1}\right)$, six age 49-52 yr $\left(\bar{x}\right.$ VO $\left._{2} \max =47 \mathrm{ml} \cdot \mathrm{kg}^{-1} \cdot \mathrm{min}^{-1}\right)$, and five age $58-63 \mathrm{yr}\left(\overline{\mathrm{x}} \mathrm{VO}_{2} \max =44 \mathrm{ml} \cdot \mathrm{kg}^{-1} \cdot \mathrm{min}^{-1}\right)$ who were matched for lean body mass. Arterial to mixed venous oxygen difference (a-vDO $\mathrm{v}_{2}$, cardiac output (Q), stroke volume (SV), left ventricular emptying time (LVET), pre-ejection period (PEP), diastolic filling time (DT), DFR, LVER, blood pressure (BP), mean arterial pressure (MAP), and total peripheral resistance (TPR) were compared at rest and during exercise, at target $\mathrm{HR}$ ( \pm 1 beat) of $110,130,150,170 \mathrm{bpm}$ and max. Resting blood volume (BV) and haemoglobin concentration ([Hb]) were also compared. As previously observed in ET males, the SV of ET females 20-29 yr increased progressively throughout incremental work rates to maximum. However, the SV of ET females $40-63$ yr did not increase beyond 
a HR of 110 bpm. Age-related decreases in HRmax, and thus, Qmax and increases in MAPmax and thus, TPRmax were responsible for this age-related difference. Further, similar to ET males, DFR far exceeded LVER throughout exercise to maximum in ET females, regardless of age. The a- $\mathrm{WDO}_{2} \max , \mathrm{BV}$, and thus, DFR, LVER and SVmax were maintained with age. However, in contrast to ET males, age-matched ET females had a smaller BV, and thus, DFRmax, LVERmax and SVmax, as well as a lower [Hb], and thus, oxygen carrying capacity and oxygen transport than ET males, which resulted in a lower $\mathrm{VO}_{2}$ max in ET females. However, when expressed relative to body mass, although $\mathrm{VO}_{2} \max ,[\mathrm{Hb}], \mathrm{BV}$, DFRmax and LVERmax were still significantly smaller in ET females, the gender differences in Qmax and SVmax were no longer significant. These results suggest that when ET males and females are matched for body mass, the gender difference in $\mathrm{VO}_{2}$ max is due to differences in [Hb], BV and thus, DFRmax and LVERmax. Further, these results suggest that when ET females are matched for lean body mass, the age-related decline in $\mathrm{VO}_{2}$ max is due to a decrease in HRmax, and thus, Qmax and an increase in MAPmax and thus, TPRmax. 


\section{ACKNOWLEDGEMENTS}

I would like to thank everyone who kindly contributed their time, energy and support to assist in the completion of this investigation.

I would especially like to thank Dr. Norman Gledhill who provided me with the opportunity to pursue my Master's degree. His expertise and vast knowledge of exercise physiology played an essential role in my learning experience. Under his supervision, I developed research, writing and presentation skills which will be invaluable in any work I may become involved in, in the future.

I would also like to thank Ms. Veronica Jamnik who offered a considerable amount of her time and energy in the laboratory. She was a constant source of help, information and encouragement during both my undergraduate and graduate studies. I will always admire her passion for teaching and learning as well as her knowledge of exercise physiology.

My sincere thanks are also extended to my laboratory peers, Mr. Steven Ferguson, Mr. Darren Warburton and Ms. Nancy Payne who generously assisted in the data collection and offered interesting insight into physiology. These individuals were not only my peers, but my friends as well.

Finally, my deepest gratitude is extended to each of the 23 women who participated in this investigation. Their cooperation and willingness to give of their time and effort will always be remembered. Without their help the completion of this project would not have been possible. 


\section{TABLE OF CONTENTS}

ABSTRACT $\ldots \ldots \ldots \ldots \ldots \ldots \ldots \ldots \ldots \ldots \ldots \ldots \ldots \ldots \ldots \ldots \ldots \ldots \ldots$, iv

ACKNOWLEDGEMENTS $\ldots \ldots \ldots \ldots \ldots \ldots \ldots \ldots \ldots \ldots \ldots$ vi

TABLE OF CONTENTS $\ldots \ldots \ldots \ldots \ldots \ldots \ldots \ldots \ldots \ldots \ldots \ldots \ldots \ldots \ldots$ vii

LIST OF FIGURES $\ldots \ldots \ldots \ldots \ldots \ldots \ldots \ldots \ldots \ldots \ldots \ldots \ldots \ldots \ldots \ldots \ldots \ldots$

LIST OF TABLES $\ldots \ldots \ldots \ldots \ldots \ldots \ldots \ldots \ldots \ldots \ldots \ldots \ldots \ldots \ldots \ldots \ldots \ldots \ldots$

SYMBOLS AND ABBREVIATIONS $\ldots \ldots \ldots \ldots \ldots \ldots \ldots \ldots \ldots \ldots \ldots \ldots \ldots$

LIST OF DEFINITIONS $\ldots \ldots \ldots \ldots \ldots \ldots \ldots \ldots \ldots \ldots \ldots \ldots \ldots \ldots \ldots \ldots \ldots$

CHAPTER I $\ldots \ldots \ldots \ldots \ldots \ldots \ldots \ldots \ldots \ldots \ldots \ldots \ldots \ldots \ldots \ldots \ldots \ldots \ldots \ldots \ldots$

INTRODUCTION $\ldots \ldots \ldots \ldots \ldots \ldots \ldots \ldots \ldots \ldots \ldots \ldots \ldots \ldots \ldots$

Statement of the Problem $\ldots \ldots \ldots \ldots \ldots \ldots \ldots \ldots \ldots \ldots \ldots$

Hypotheses $\ldots \ldots \ldots \ldots \ldots \ldots \ldots \ldots \ldots \ldots \ldots \ldots \ldots \ldots \ldots \ldots \ldots, 8$

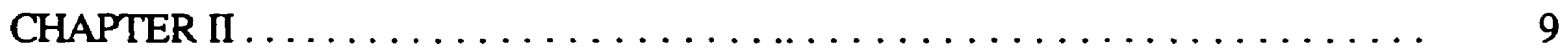

REVIEW OF THE LITERATURE $\ldots \ldots \ldots \ldots \ldots \ldots \ldots \ldots \ldots \ldots$

Introduction $\ldots \ldots \ldots \ldots \ldots \ldots \ldots \ldots \ldots \ldots \ldots \ldots \ldots \ldots \ldots \ldots, 9$

Maximal Oxygen Uptake and the Relationship to Gender and Age ...... 9

Cardiac Output and Stroke Volume and the Relationship to Gender and Age 25

Cardiac Time Intervals and the Relationship to Gender and Age ....... 56

Blood Volume and the Relationship to Gender and Age ........... 64 
viii

Diastolic Filling and Left Ventricular Ejection and the Relationship to

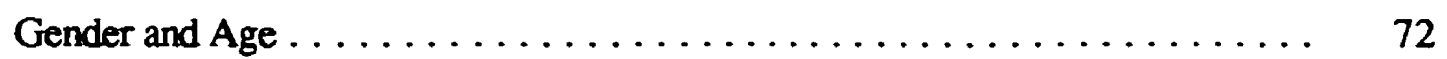

SUMMARY OF REVIEW OF LITERATURE $\ldots \ldots \ldots \ldots \ldots \ldots \ldots .84$

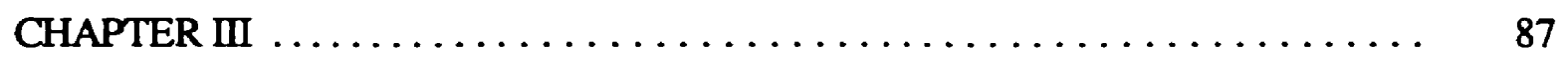

METHODS AND PROCEDURES $\ldots \ldots \ldots \ldots \ldots \ldots \ldots \ldots \ldots \ldots \ldots$

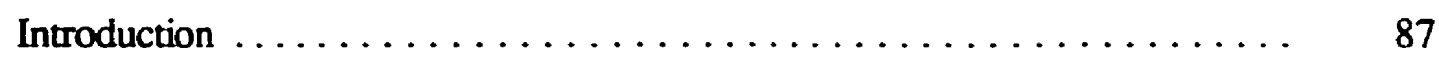

Subjects $\ldots \ldots \ldots \ldots \ldots \ldots \ldots \ldots \ldots \ldots \ldots \ldots \ldots \ldots \ldots \ldots \ldots \ldots \ldots$

Experimental Design $\ldots \ldots \ldots \ldots \ldots \ldots \ldots \ldots \ldots \ldots \ldots \ldots \ldots \ldots \ldots$

Test Protocol $\ldots \ldots \ldots \ldots \ldots \ldots \ldots \ldots \ldots \ldots \ldots \ldots \ldots \ldots \ldots$

Determination of Heart Rate $\ldots \ldots \ldots \ldots \ldots \ldots \ldots \ldots \ldots \ldots$

Determination of Oxygen Uptake. . . . . . . . . . . . . $\quad 90$

Determination of Cardiac Time Intervals . . . . . . . . . . . 92

i) Ear Pulse Densitography Measurement. . . . . . . . 92

ii) Phonocardiogram Measurements $\ldots \ldots \ldots \ldots \ldots \ldots \ldots \ldots \ldots .93$

iii) Calculation of Cardiac Time Intervals . . . . . . . . . . . 93

Determination of Blood Pressure . . . . . . . . . . . . . . . . 95

Determination of Cardiac Output via the Acetylene Rebreathe Method .... . 96

i) Calculation of Cardiac Output $\ldots \ldots \ldots \ldots \ldots \ldots \ldots \ldots \ldots \ldots \ldots$

Determination of Diastolic Filling Rate and Left Ventricular Ejection Rate . . 99

Hematology Measurements . . . . . . . . . . . . . . . . . . . . 99 
i) Measurement of Haemoglobin $\ldots \ldots \ldots \ldots \ldots \ldots \ldots \ldots \ldots, 100$

ii) Measurement of Haematocrit $\ldots \ldots \ldots \ldots \ldots \ldots \ldots \ldots, 100$

Determination of Blood Volume $\ldots \ldots \ldots \ldots \ldots \ldots \ldots \ldots \ldots \ldots, 101$

Anthropometric Measurements . . . . . . . . . . . . . . . 102

Determination of Lung Volume $\ldots \ldots \ldots \ldots \ldots \ldots \ldots \ldots \ldots \ldots$

Statistical Analyses $\ldots \ldots \ldots \ldots \ldots \ldots \ldots \ldots \ldots \ldots \ldots \ldots \ldots \ldots \ldots$

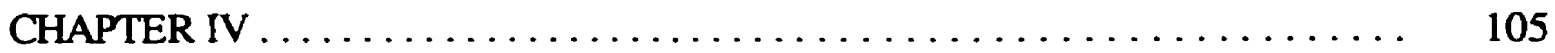

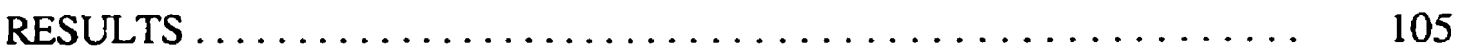

Participant Characteristics .......................... 105

Effects of Age on Cardiac Function . . . . . . . . . . 107

Oxygen Uptake . . . . . . . . . . . . . . . . . . . . . 107

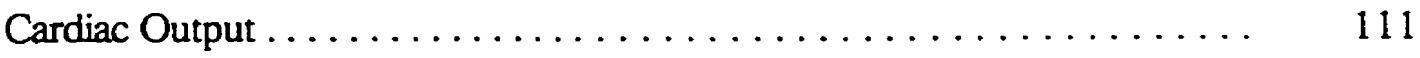

Stroke Volume $\ldots \ldots \ldots \ldots \ldots \ldots \ldots \ldots \ldots \ldots \ldots \ldots \ldots, \quad 117$

Arterial-Mixed Venous Oxygen Difference ................ 117

Cardiac Time Intervals .......................... 117

Diastolic Filling Rate and Left Ventricular Ejection Rate ......... 125

Blood Pressure, Mean Arterial Pressure and Total Peripheral Resistance .... 128

SUMMARY OF RESULTS $\ldots \ldots \ldots \ldots \ldots \ldots \ldots \ldots \ldots \ldots \ldots, 141$ 


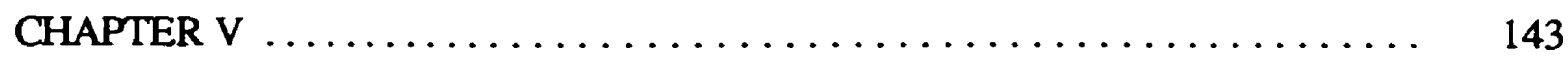

DISCUSSION $\ldots \ldots \ldots \ldots \ldots \ldots \ldots \ldots \ldots \ldots \ldots \ldots \ldots \ldots \ldots \ldots \ldots$

Maximal Aerobic Capacity in ET Individuals and the Relationship to Age

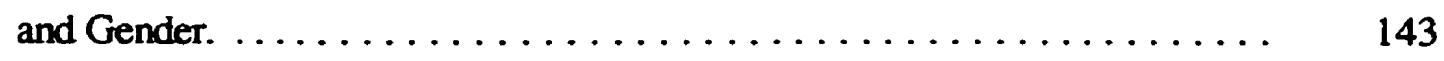

Cardiac Output in ET Individuals and the Relationship to Age and Gender. . . 149

Stroke Volume in ET Individuals and the Relationship to Age and Gender. . 153

Arterial-Mixed Venous $\mathrm{O}_{2}$ Difference in ET Individuals and the Relationship

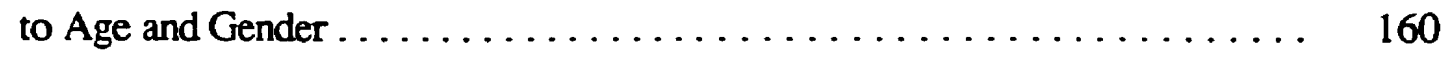

Cardiac Time Intervals in ET individuals and the Relationship to Age

and Gender $\ldots \ldots \ldots \ldots \ldots \ldots \ldots \ldots \ldots \ldots \ldots \ldots \ldots \ldots \ldots \ldots \ldots \ldots \ldots \ldots$

Blood Volume in ET Individuals and the Relationship to Age and Gender . . 171

Diastolic Filling Rate and Left Ventricular Ejection Rate in ET Individuals

and the Relationship to Age and Gender $\ldots \ldots \ldots \ldots \ldots \ldots \ldots \ldots \ldots, 174$

Blood Pressure, MAP and TPR in ET Individuals and the Relationship to

Age and Gender $\ldots \ldots \ldots \ldots \ldots \ldots \ldots \ldots \ldots \ldots \ldots \ldots \ldots \ldots \ldots \ldots$

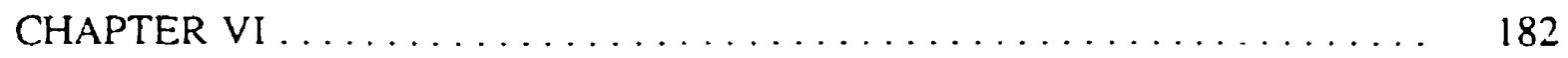

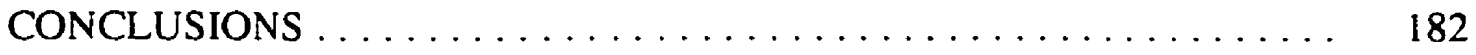

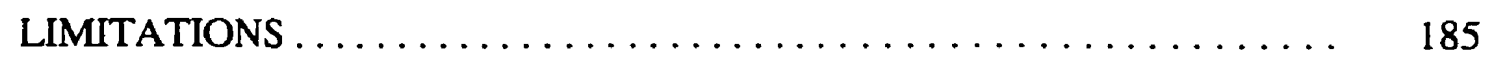

RECOMMENDATIONS $\ldots \ldots \ldots \ldots \ldots \ldots \ldots \ldots \ldots \ldots \ldots \ldots \ldots$ 


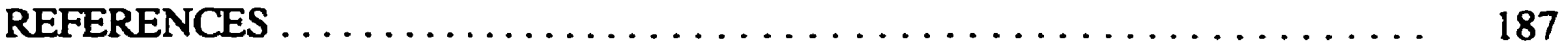

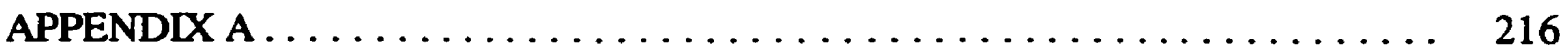

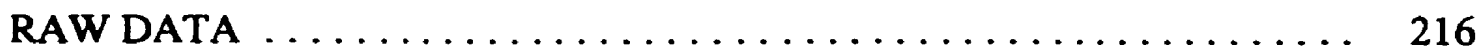

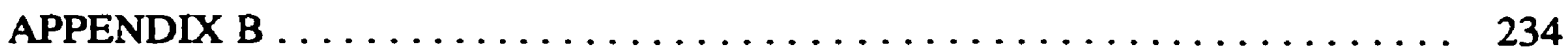

DETERMINATION OF OXYGEN UPTAKE $\ldots \ldots \ldots \ldots \ldots \ldots \ldots \ldots .234$

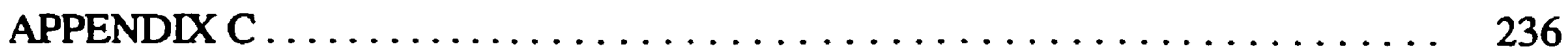

DETERMINATION OF a-v̄DO ${ }_{2}$ FROM THE ACETYLENE

REBREATHE TECHNIQUE $\ldots \ldots \ldots \ldots \ldots \ldots \ldots \ldots \ldots \ldots \ldots \ldots \ldots \ldots \ldots$

APPENDIX D . . . . . . . . . . . . . . . . . . . . . . . . . 238

DETERMINATION OF PLASMA AND BLOOD VOLUME $\ldots \ldots \ldots \ldots .238$

APPENDIX E. ..................................... 240

DETERMINATION OF BODY DENSITY AND PERCENT BODY FAT . . . 240

APPENDIX F................................... 242

INFORMED CONSENT DOCUMENT . . . . . . . . . . . . . . . 242 
xii

\section{LIST OF FIGURES}

EIGURE

PAGE

1 Diagram illustrating the calculations of LVET from the ear pulse densitographic tracings and PEP and DT from the ECG and heart sound tracings ...... 94

2 Diagram illustrating the oxygen and acetylene gas decay curves used in the calculation of $\mathrm{a}-\mathrm{xDO}_{2} \ldots \ldots \ldots \ldots \ldots \ldots \ldots \ldots \ldots \ldots \ldots \ldots \ldots$

3 Resting blood volume in absolute and relative terms as a function of group .. 108

4 Oxygen uptake $\left(1 \cdot \mathrm{min}^{-1}\right)$ as a function of HR and group. . . . . . . . 109

5 Oxygen uptake $\left(\mathrm{ml} \cdot \mathrm{kg}^{-1} \cdot \mathrm{min}^{-1}\right)$ as a function of $\mathrm{HR}$ and group. . . . . . 110

6 Cardiac output as a function of $\mathrm{HR}$ and group............... 112

$7 \quad$ Cardiac output as a function of oxygen uptake and group.......... 113

$8 \quad$ Stroke volume as a function of $\mathrm{HR}$ and group $\ldots \ldots \ldots \ldots \ldots \ldots \ldots, 115$

9 Stroke volume as a function of oxygen uptake and group $\ldots \ldots \ldots \ldots, 116$

10 Arterial-mixed venous oxygen difference as a function of HR and group. .. 118

11 Arterial-mixed venous oxygen difference as a function of oxygen uptake

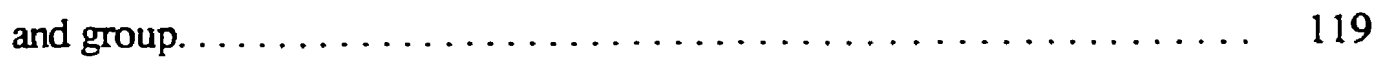

12 Left ventricular ejection time as a function of $H R$ and group. ....... 121

13 Pre-ejection period as a function of HR and group. . . . . . . . . . 122

14 Diastolic time as a function of $\mathrm{HR}$ and group. .............. 123

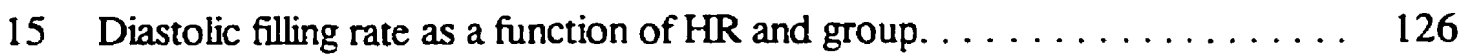


16 Left ventricular ejection rate as a function of HR and group. . . . . . . 127

17 Systolic blood pressure as a function of $\mathrm{HR}$ and group. . . . . . . . 129

18 Systolic blood pressure as a function of the percentage of maximal oxygen uptake and group $\ldots \ldots \ldots \ldots \ldots \ldots \ldots \ldots \ldots \ldots \ldots \ldots \ldots \ldots \ldots \ldots$

19 Diastolic blood pressure as a function of $\mathrm{HR}$ and group. .......... 132

20 Diastolic blood pressure as a function of the percentage of maximal oxygen uptake and group. $\ldots \ldots \ldots \ldots \ldots \ldots \ldots \ldots \ldots \ldots \ldots \ldots \ldots \ldots$

21 Mean arterial pressure as a function of HR and group........... 135

22 Mean arterial pressure as a function of the percentage of maximal oxygen uptake

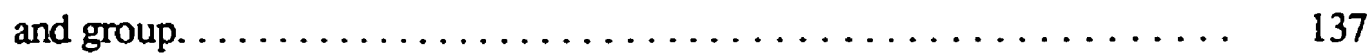

23 Total peripheral resistance as a function of $\mathrm{HR}$ and group. . . . . . . 138

24 Total peripheral resistance as a function of the percentage of maximal oxygen

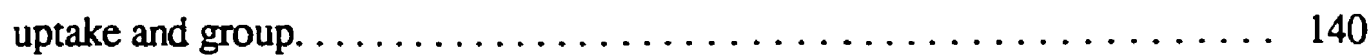


xiv

\section{LIST OF TABLES}

TABLE

PAGE

1 Symbols and Abbreviations. ....................

2 Summary of participants' characteristics and cardiac function $\left(\mathrm{VO}_{2}, \mathrm{Q}, \mathrm{SV}\right.$, a-vDO ${ }_{2}$, CTI (LVET, PEP, DT), DFR, LVER, SBP, DBP, MAP, and TPR) 106

\section{APPENDIX TABLES}

\section{A 1-17 RAW DATA}




\section{SYMBOLS AND ABBREVIATIONS}

The following table contains a list of symbols and abbreviations used throughout this thesis.

TABLE 1. Symbols and Abbreviations

\begin{tabular}{ll} 
Symbol & \multicolumn{1}{c}{ Definition } \\
AR & adrenergic receptor \\
a- $-\mathrm{vDO}_{2}$ & arterial-mixed venous oxygen difference \\
a- $-\mathrm{vDO}_{2} \mathrm{max}$ & maximal arterial-mixed venous oxygen difference \\
$\beta-\mathrm{AR}$ & beta adrenergic receptor \\
$\mathrm{BP}$ & blood pressure \\
bpm & beats per minute \\
$\mathrm{BV}$ & blood volume \\
$\mathrm{C} \mathrm{H}_{2}$ & acetylene \\
$\left(\mathrm{C}_{2} \mathrm{H}_{2}\right)_{\mathrm{c}}$ & corrected acetylene \\
$\left(\mathrm{C}_{2} \mathrm{H}_{2}\right)_{\mathrm{dir}}$ & acetylene difference \\
$\mathrm{CI}$ & cardiac index \\
$\mathrm{CO}$ & carbon dioxide \\
$\mathrm{CTI}$ & cardiac time intervals \\
$\mathrm{DBP}$ & diastolic blood pressure \\
$\mathrm{DBPmax}$ & maximal diastolic blood pressure \\
$\mathrm{DFR}$ & distolic filling rate \\
\hline
\end{tabular}


DFRmax maximal distolic filling rate

DT diastolic filling time

DTmax maximal diastolic filling time

ECG electrocardiogram

EDV end-diastolic volume

EDVI end-diastolic volume index

ESV end-systolic volume

ESVI end-systolic volume index

EF ejection fraction

ERT estrogen replacement therapy

ET endurance trained

Q-S $\quad$ electromechanical systole

EPD ear pulse densitograph

$\mathrm{F}_{\mathrm{E}} \mathrm{CO}_{2}$ fractional concentration of expired carbon dioxide

$\mathrm{F}_{\mathrm{E}} \mathrm{N}_{2} \quad$ fractional concentration of expired nitrogen

$\mathrm{F}_{\mathrm{E}} \mathrm{O}_{2} \quad$ fractional concentration of expired oxygen

$\mathrm{F}_{1} \mathrm{CO}_{2} \quad$ fractional concentration of inspired carbon dioxide

$\mathrm{F}_{\mathrm{l}} \mathrm{N}_{2} \quad$ fractional concentration of inspired nitrogen

$\mathrm{F}_{\mathrm{r}} \mathrm{O}_{2} \quad$ fractional concentration of inspired oxygen

FVC forced vital capacity

[Hb] concentration of haemoglobin 
xvii

\begin{tabular}{|c|c|}
\hline Hct & haematocrit \\
\hline HR & heart rate \\
\hline HRmax & maximal heart rate \\
\hline HRT & hormone replacement therapy \\
\hline LBM & lean body mass \\
\hline LV & left ventricle \\
\hline LVEDD & left ventricular end-diastolic diameter \\
\hline LVEDV & left ventricular end-diastolic volume \\
\hline LVER & left ventricular ejection rate \\
\hline LVERmax & maximal left ventricular ejection rate \\
\hline LVESV & left ventricular end-systolic volume \\
\hline LVET & left ventricular ejection time \\
\hline LVETmax & maximal left ventricular ejection time \\
\hline MAP & mean arterial pressure \\
\hline MAPmax & maximal mean arterial pressure \\
\hline $\mathrm{mmHg}$ & millimetres of mercury \\
\hline msec & milliseconds \\
\hline MVV & maximal ventilatory volume \\
\hline $\mathbf{N}_{2}$ & nitrogen \\
\hline $\mathbf{N A}$ & normally active \\
\hline $\mathrm{O}_{2}$ & oxygen \\
\hline
\end{tabular}


xviii
$\left(\mathrm{O}_{2}\right)_{c} \quad$ corrected oxygen
$\left(\mathrm{O}_{2}\right)_{\text {dif }} \quad$ oxygen difference
OCA oral contraceptive agents
$\mathrm{Pb} \quad$ barometric pressure
PCG phonocardiogram
PEP pre-ejection period
PEPmax maximal pre-ejection period
PV plasma volume
Q $\quad$ cardiac output
Qmax maximal cardiac output
Q-S $\quad$ total electromechanical systole
RCV red cell volume
RBC red blood cells
RR respiratory rate
RV residual volume
$\mathrm{S}_{2} \quad$ second heart sound
SBP systolic blood pressure
SBPmax maximal systolic blood pressure
SEM standard error of the mean
STPD standard temperature, pressure, dry $(0 \mathrm{C}, 760 \mathrm{mmHg}$, no pressure due to water vapour) 


$\begin{array}{ll}\text { SV } & \text { stroke volume } \\ \text { SVI } & \text { stroke volume index } \\ \text { SVmax } & \text { maximal stroke volume } \\ \text { TLC } & \text { total lung capacity } \\ \text { TPR } & \text { total peripheral resistance } \\ \text { TPRmax } & \text { maximal total peripheral resistance } \\ \mathrm{TPFR} & \text { time to peak filling rate } \\ \mathrm{TV} & \text { tidal volume } \\ \mathrm{V}_{\mathrm{E}} & \text { ventilation } \\ \mathrm{VO}_{2} & \text { oxygen uptake } \\ \mathrm{VO}_{2} \mathrm{max} & \text { maximal oxygen uptake } \\ \overline{\mathrm{x}} & \text { mean } \\ \overline{\mathrm{x}} & \end{array}$




\section{LIST OF DEEINITIONS}

The following list of terms and definitions are used frequently throughout this thesis.

Afterload is the impedence or resistance to the ejection of blood (ventricular emptying) and effects ventricular ejection fraction and myocardial fibre shortening velocity.

Alpha-adrenergic receptors are plasma membrane receptors for epinephrine and norepiniphrine and are located on the blood vessels.

Arterial oxygen content is the amount of oxygen bound to hemoglobin and dissolved in the blood $\left(\mathrm{ml} \mathrm{O}_{2} \cdot 100 \mathrm{ml} \mathrm{blood}^{-1}\right)$.

Arterial oxygen transport is the amount of oxygen in the blood that is available to be transported to the tissues and $\left(\mathrm{O}_{2} \cdot 100 \mathrm{~min}^{-1}\right)$.

Arterial to mixed venous oxygen difference is the difference in oxygen content between arterial and mixed venous blood $\left(\mathrm{ml} \mathrm{O}_{2} \cdot 100 \mathrm{ml} \mathrm{blood}^{-1}\right)$, that reflects the amount of oxygen used by the tissues.

Beta-adrenergic receptors are plasma membrane receptors for norepinephrine and epinephrine and are located on the sino-atrial node and ventricle of the heart and on the blood vessels.

Blood volume is the total amount of tluid contained in the systemic circulation and is composed of plasma and red blood cells.

Cardiac cycle is the period that contains all the events between two consecutive heart beats. Cardiac output is the volume of blood pumped by the heart per minute $\left(1 \cdot \mathrm{min}^{-1}\right)$ and refers 
$\mathbf{x x i}$

to the product of the heart rate and stroke volume.

Catecholamines are hormones, such as epinephrine and norepinephrine which effect the heart rate and force of contraction and the blood vessel tone.

Cross-sectional investigations are those in which different individuals of varying ages are compared ie., highly trained masters athletes compared to younger athletes.

Dehydration refers to a loss of water and electrolytes.

Diastole is the period of the cardiac cycle when the ventricles relax and fill.

Diastolic blood pressure is the lowest arterial pressure during diastole ( $\mathrm{mmHg}$ ).

Diastolic filling rate is the volume of blood which fills the ventricles in a given period of time $\left(\mathrm{ml} \cdot \mathrm{sec}^{-1}\right)$ and is calculated as the stroke volume (ml) divided by the diastolic time (msec).

Diastolic time refers to the time period of the cardiac cycle from the closure of the aortic valve $\left(\mathrm{S}_{2}\right)$ to the onset of ventricular excitation of the next heart beat (msec).

Ejection fraction refers to the percentage of end-diastolic volume which is ejected from the left ventricle with each contraction, and is calculated as the stroke volume divided by the enddiastolic volume.

End-diastolic volume is the volume of blood $(\mathrm{ml})$ inside the left ventricle at the end of diastole, prior to contraction.

End-systolic volume is the volume of blood (ml) remaining inside the left ventricle at the end of systole, after contraction.

Estrogen is the major female sex hormone and is secreted by the ovaries. 
xxii

Estrogen Replacement Therapy refers to drugs composed of exogenous estrogen which may be prescribed to pre-menopausal ammenorheic females or post-menopausal females to prevent bone loss.

Eumenorthea refers to normal menstrual function which consists of a menstrual cycle which lasts from 23 to $35 \mathrm{~d}$ with 11 to 14 cycles per year.

Follicular Phase refers to the time period of the menstrual cycle lasting from the first day of menstruation until ovulation, and lasts for an average of $\sim 14 \mathrm{~d}$. During the first few days of this phase $(-1-8 \mathrm{~d})$, levels of the female sex hormones (estrogen and progesterone) are low. Forced vital capacity is the volume of gas (l) that can be maximally expired by voluntary effort after maximal inspiration.

Frank-Starling mechanism refers to the cardiac response in which an increased end-diastolic volume results in a stronger ventricular contraction, which in turn, increases the volume of blood ejected.

Heart rate is the rate at which the heart is contracting (beats· $\mathrm{min}^{-1}$ ).

Haematocrit is the percentage of red blood cells in the total blood volume.

Haemoconcentration is a relative increase in the concentration of red blood cells, due to a decrease in plasmal volume.

Haemodilution is a relative dilution of haematocrit and haemoglobin, due to an increase in plasma volume.

Haemoglobin is an iron containing protein in red blood cells which binds with oxygen. 
xxiii

Hormone Replacement Therapy refers to drugs composed of exogenous female sex hormones (estrogen and progesterone) which are prescribed to post-menopausal females. The estrogen aids in the prevention of bone loss and the progesterone helps to prevent endometrial proliferation.

Hyperventilation is an increased ventilation without similar increases in carbon dioxide production.

Isovolumic relaxation time refers to the time period of the cardiac cycle after aortic valve closure, prior to the decrease in ventricular pressure, which allows the mitral valve to open. Left ventricular ejection rate is the volume of blood ejected by the left ventricle in a given time $\left(\mathrm{ml} \cdot \mathrm{sec}^{-1}\right)$ and is calculated as the stroke volume $(\mathrm{ml})$ divided by the left ventricular ejection time (msec).

Left ventricular ejection time refers to the time period of the cardiac cycle when the left ventricle ejects blood into the arterial system (msec).

Longitudinal investigations examine repeated measures within the same individual at different parts of the life span ie., sedentary individuals undergo nine months of endurance training and have their pre-training responses examined and compared to their post-training responses.

Luteal Phase refers to the time period of the menstrual cycle lasting from ovulation until the onset of the next menstrual period, and lasts for $\sim 14 \mathrm{~d}$. During the luteal phase, levels of the female sex hormones (estrogen and progesterone) are high. 
xxiv

Maximal oxygen uptake refers to the volume of oxygen consumed by the body during maximal exercise $\left(1 \cdot \mathrm{min}^{-1}\right.$ or $\left.\mathrm{ml} \cdot \mathrm{kg}^{-1} \cdot \mathrm{min}^{-1}\right)$ and is equal to the product of cardiac output times the aterial to mixed venous oxygen difference.

Mean arterial pressure is the average blood pressure $(\mathrm{mmHg})$ during the cardiac cycle and is calculated as diastolic blood pressure $+1 / 3$ (systolic pressure - diastolic pressure).

Menopause refers to the loss of cyclic hormonal function occuring at the average age of 50 $\mathrm{yr}$ in females and results in the cessation of menstrual cycling.

Menstrual cycle is the cyclical rise and fall in female reproductive hormones and processes, averaging 28 days and consisting of a luteal phase and a follicular phase.

Oral contraceptive agents are drugs composed of exogenous female sex hormones (estrogen and progesterone) which are used for birth control and other medical purposes.

Oxygen uptake is the rate at which the body can produce ATP aerobically $\left(1 \cdot \mathrm{min}^{-1}\right.$ or $\mathrm{ml}$ $\left.\mathrm{kg}^{-1} \cdot \min ^{-1}\right)$.

Plasma volume is the liquid portion of the blood and is composed of water, electrolytes, hormones, enzymes, cellular nutrients, wastes, respiratory gases and proteins (ie. albumin). Pre-ejection period refers to the time period of the cardiac cycle from the onset of depolarization of the myocardium to the start of ventricular contraction (msec).

Preload is the extent of ventricular filling or the end-diastolic fibre length before contraction. Progesterone is a female sex hormone and is secreted by the ovaries.

Residual volume is the volume of gas (ml) remaining in the lungs after maximal expiration. 
Stroke volume is the amount of blood ejected from the left ventricle (ml) during a contraction and refers to the difference between the end-diastolic volume and the end-systolic volume.

Systole is the period of the cardiac cycle when the ventricles contract.

Systolic blood pressure is the highest arterial blood pressure during systole (mmHg).

Total electromechanical systole is the onset of ventricular excitation ( $Q$ wave) to the end of systole when the aortic valve closes $\left(S_{2}\right)$ and is expressed in msec.

Total peripheral resistance is the total resistance to flow in the systemic blood vessels $\left(\mathrm{mmHg} \cdot \mathrm{l}^{-1} \cdot \mathrm{min}^{-1}\right)$ and is calculated as the mean arterial pressure $(\mathrm{mmHg})$ divided by the cardiac output $\left(1 \cdot \mathrm{min}^{-1}\right)$. 


\section{CHAPTER I}

\section{INTRODUCTION}

Maximal oxygen uptake $\left(\mathrm{VO}_{2} \mathrm{max}\right)$ is a key determinant of cardiorespiratoy endurance since it sets the upper limit of an individuals' rate of aerobic metabolism. Chronic endurance training results in cardiorespiratory adaptations which enhance cardiac function and $\mathrm{VO}_{2}$ max. A high $\mathrm{VO}_{2}$ max depends upon the ability of the cardiovascular and respiratory systems to deliver a large amount of oxygenated blood to the exercising muscles in a short period of time. This oxygen delivery in turn depends on both maximal cardiac output ( $Q \max )$ and the capacity of the muscles to extract and utilize the oxygen, as reflected in the maximal arterial to mixed venous oxygen difference $\left(\mathrm{a}-\mathrm{ZDO}_{2} \mathrm{max}\right)$. The cardiovascular and respiratory systems which determine oxygen transport are referred to as central, while the local tissue oxygen extraction and utilization are referred to as peripheral. The relationship between these components is described by the Fick equation: $\mathrm{VO}_{2}=\mathrm{Q} \times \mathrm{a}-\mathrm{v} D Q$. Thus, an increased $\mathrm{VO}_{2} \max$ may result from an increased $\mathrm{Qmax}$ and/or an increased a- $\mathrm{vDO}_{2} \max$.

Both Qmax and maximal stroke volume (SVmax) are significantly larger in younger (7, $11,67,68,105,152,308,309)$ and older endurance trained (ET) males $(117,122,205,222$, 234) as well as in younger and older ET females (205), compared to age and gender-matched normally active (NA) individuals. Peripheral adaptations in active skeletal muscle also play an important role in the increase in $\mathrm{VO}_{2} \max$ as a result of endurance training. For instance, younger $(11,240)$ and older ET males $(205,222)$, as well as younger (205) and older ET females (205) have a larger a- $\overline{\mathrm{v}} \mathrm{DO}_{2} \max$ than age and gender-matched NA individuals. 
In males, endurance training has a greater effect on $Q \max$ than on $\mathrm{a}-\overline{\mathrm{vDO}}{ }_{2} \max (11,68$, $121,147)$. For example, it has been reported that $50-100 \%$ of the higher $\mathrm{VO}_{2}$ max seen in both younger $(7,68,105,309)$ and older $(117,122,205,222)$ ET males compared to agematched NA males, is due to a greater Qmax, resulting from an elevated SVmax. Further, an enhanced SV may result from an increased left ventricular end-diastolic volume (LVEDV) and/or a decreased left ventricular end-systolic volume (LVESV) (since SV = LVEDV LVESV). Mechanisms responsible for an increased LVEDV are an increased preload and myocardial hypertrophy, whereas, a decreased LVESV has been attributed to enhanced myocardial contractility and decreased afterload $(11,70,128)$.

However, contraversy exists regarding whether it is enhanced myocardial contractility or enhanced preload (Frank-Starling mechanism) that is primarily responsible for augmenting SVmax. It has generally been reported that the SV of both ET and NA males increases until it reaches a plateau at exercise intensities between 40 and $60 \%$ of $\mathrm{VO}_{2} \max (7,104,108,123$, 211,268 ). It was speculated that at heart rates (HR) above $170 \mathrm{bpm}$, a reduced diastolic filling time (DT), limits ventricular filling and thus, end-diastolic volume (EDV), resulting in a levelling off of SV $(7,104,108,123,211,268)$. These researchers postulated that the Frank-Starling mechanism has its greatest influence at low to moderate work rates while myocardial contractility has its greatest influence at higher work rates $(7,104,108,123,211$, 268). Therefore, any further increases in $Q$ beyond $40-60 \%$ of $\mathrm{VO}_{2} \max$ would be due to increases in HR and myocardial contractility.

However, contrary to this view, investigators have recently shown that ET males have 
the ability to effectively use the Frank-Starling mechanism up to $\mathrm{VO}_{2} \max (46,105,128,152$, $165,220,230$ ). These investigators observed a progressive increase in SV throughout incremental exercise to maximum without a plateau in ET males $(46,67,68,105,152,220$, $222,285,289$ ). The progressive increase in SV to maximum in ET males has been associated with enhancements in both the diastolic filling rate (DFR) and left ventricular emptying rate (LVER) $(33,71,105,152,167,186)$. By examining SV and cardiac time intervals (CTI) simultaneously, it has been demonstrated that the primary advantage of ET over NA males is in the DFR $(105,152)$. The enhanced DFR was linked to an augmented blood volume (BV) in ET males $(35,105,152,289)$. Furthermore, in a recent investigation from our laboratory, it was concluded that a large portion of the enhanced cardiac function in ET compared to NA males is a passive response to a higher BV in ET males, which in turn favours adequate ventricular filling when DT shortens at high-HR, enabling SV to continue to increase up to maximal exercise via the Frank-Starling mechanism (152). Further, ET males required a longer left ventricular emptying time (LVET) and a shorter DT than NA males $(105,152)$. However, since SV and CTI have not previously been measured simultaneously in ET females during incremental exercise to maximum, it remains to be determined whether differences exist between the SV response and CTI in ET females compared to ET males, and also whether there are any changes in these variables with aging.

It has been well documented that $\mathrm{VO}_{2} \max$ and $\mathrm{HRmax}$ decline progressively with advancing age regardless of gender or training status $(6,22,54,60,65,66,83,102,117$, $122,130,180,132,205,214,222,226)$. However, contraversy exists regarding the age- 
related changes in $Q$ during exercise. While some investigators have observed age-related reductions in exercise $Q(81,110,117,122,124,139,205,222,267,318)$ others have observed no changes in exercise $Q$ with aging $(8,100,224)$. The role of $S V \max$ and a$\mathrm{DDO}_{2}$ max in explaining the age-related decline in $\mathrm{VO}_{2} \max$ in both males and females is also controversial $(81,82,100,117,122,205,222,224,245,318)$. Some researchers examining older ET males and females suggest that, compared to their younger ET counterparts, the decline in $\mathrm{VO}_{2} \max$ is due solely to a decrease in $\mathrm{HRmax}(117,122)$, while other investigators propose a contribution of only $25-40 \%$ from this mechanism $(205,222)$.

Further, older ET males and females are better able to maintain lean body mass (LBM) and tend to have a smaller amount of subcutaneous fat than age and gender-matched NA individuals $(122,180,205,214)$, which together should result in a larger $\mathrm{VO}_{2} \max$ in both $1 \cdot \mathrm{min}^{-1}$ and $\mathrm{ml} \cdot \mathrm{kg}^{-1} \cdot \mathrm{min}^{1}$. In addition, some investigators have shown smaller declines in HRmax and/or a-iDO ${ }_{2} \max$ in ET males and females, which was associated with a lesser rate of decline in $\mathrm{VO}_{2} \max$ compared to age and gender-matched NA individuals $(117,122,205$, 226). However, since there are a limited number of investigations to date in which the mechanisms responsible for the age-related decline in $\mathrm{VO}_{2} \max$ in ET females have been examined (205), it remains to be determined which of the factors are of primary importance.

It has also been well documented that the early DFR declines as a function of age in NA males $(4,12,18,97,101,97,148,193,196,237,261)$. However, contraversy exists regarding the age-related changes in DFR in ET individuals. While some investigators have observed no difference in the DFR of older ET males compared to age-matched NA males 
$(81,243)$, others show an attenuation or reversal of this phenomena with endurance training $(62,85,167,256)$. Since no data have been reported regarding the age-related changes in DFR in ET females, their age-related responses at rest and during exercise remain to be determined. 


\section{Statement of the Problem}

Although there is mounting evidence to support an increase in SV throughout incremental exercise to maximum in ET males, it is unknown whether this adaptation occurs in ET females. Simultaneous measurements of SV and CTI in ET females have not previously been reported. Thus, it is unknown whether they change throughout incremental exercise to maximum in a manner previously observed in $E T$ males. Also, research on training-induced changes in DFR and LVER in females during incremental work rates to maximum requires investigation. In addition, the role that an elevated BV and thus, preload, plays in the SV responses and the DFR and LVER measured in both younger and older ET females is unknown. Further, the influence of aging on the relationship between SV responses and CTI changes throughout incremental exercise to maximum has not previously been studied in ET females. Lastly, since SV is also affected by afterload, which in turn is influenced by blood pressure (BP), BP needs to be measured and compared between groups.

Therefore, the purposes of this study were to examine the a-vDO ${ }_{2}, Q, S V, L V E T$, preejection period (PEP), DT, DFR, LVER, BP, mean arterial pressure (MAP) and total peripheral resistance (TPR) of ET females age i) 20-29 yr, ii) 40-45 yr, iii) 49-54 yr, and iv) 58.63 yr at matched HR during incremental exercise to maximum. In addition, BV and haemoglobin concentration $([\mathrm{Hb}])$ were determined and compared between groups.

Since the cardiovascular and respiratory systems represent possible limiting factors for both $\mathrm{VO}_{2}$ max and one's ability to sustain submaximal levels of physical activity, and since there is a tendency towards age-related reductions in the functional reserves of these systems 
in healthy individuals, it is important to understand possible interactions between endurance training and cardiac function in response to aging. 


\section{Hypotheses}

It was hypothesized that:

1) The SV of ET females would increase progressively, throughout incremental work rates up to maximum.

2) Compared to participants 20-29 yr, those 40-45 yr would have a longer LVET and a shorter DT both at rest and at matched HR during exercise.

3) Compared to participants $40-45 \mathrm{yr}$, those $49-54 \mathrm{yr}$ would have a longer LVET and a shorter DT both at rest and at matched HR during exercise.

4) Compared to participants 49-54 yr, those 58-63 yr would have a longer LVET and a shorter DT both at rest and at matched HR during exercise.

5) The rates of ventricular filling and emptying of ET females 20-29 yr, 40-45 yr, 49-54 yr and 58-63 yr would be similar both at rest and at matched HR during exercise.

6) The BP, MAP and TPR responses of ET females 20-29 yr, 40-45 yr, 49-54 yr and 58-63 yr would be similar both at rest and at the same relative (\% of maximum) exercise intensities.

7) The BV (ml- $\left.\mathrm{kg}^{-\mathrm{l}}\right)$ of ET females 20-29 yr, 40-45 yr 49-54 yr and 58-63 yr would be similar. 


\section{CHAPTER II}

\section{REVIEW OF THE LTCERATURE}

\section{Introduction}

Endurance training results in both central and peripheral adaptations which together enhance an individual's capacity to perform maximal aerobic work $(11,240)$. In particular, endurance training results in enhancements in cardiac function, which in turn, lead to an increased ability to deliver oxygen to the exercising muscles.

This chapter contains a review of the literature pertaining to the adaptations in cardiac function which occur as a result of endurance training. Also covered are investigations in which the differences in cardiac function between ET males and females were examined, as well as studies in which cardiac function changes that occur with aging in ET males and females were explored. In addition, the roles between BV, SV, CTI, DFR, LVER and TPR in ET individuals at rest and during both submaximal and maximal exercise are examined. Maximal Oxygen Uptake in ET Individuals and the Relationship to Gender and Age Maximal Oxygen Uptake in ET Compared to NA Individuals

A significantly larger $\mathrm{VO}_{2}$ max has consistently been observed in ET younger $(5,42)$ and older males $(54,60,115,205,117,122)$ as well as in ET younger $(71,98,181,205,207$. $213,280,306,319)$, and older females $(205,265,280,281,282,283,290,295)$ compared to age and gender-matched $\mathrm{NA}$ individuals. In males, $\mathrm{VO}_{2}$ max values have been reported as high as $94 \mathrm{ml} \cdot \mathrm{kg}^{-1} \cdot \mathrm{min}^{-1}$ in a younger Nordic skier (5) with averages of $\sim 70 \mathrm{ml} \cdot \mathrm{kg}^{-1} \cdot \mathrm{min}^{-1}$ (42) compared to $\sim 42-50 \mathrm{ml} \cdot \mathrm{kg}^{-1} \cdot \mathrm{min}^{-1}$ in NA age-matched males $(5,144)$. In females, $\mathrm{VO}_{2} \max$ 
values have been observed as high as $77 \mathrm{ml} \cdot \mathrm{kg}^{-1} \cdot \mathrm{min}^{-1}$, in a younger Nordic Skier (5) and 73 $\mathrm{ml} \cdot \mathrm{kg}^{-1} \cdot \mathrm{min}^{-1}$, in an elite middle-distance runner (65), with averages of $\sim 68 \mathrm{ml} \cdot \mathrm{kg}^{\mathrm{k}} \cdot \mathrm{min}$ (207). Other investigators have reported more conservative averages of $\sim 55-62 \mathrm{ml} \cdot \mathrm{kg}^{-1} \cdot \mathrm{min}$ in younger ET females $(52,98,181,205,280,306,319)$, compared to $\sim 35-40 \mathrm{ml} \cdot \mathrm{kg}^{-1} \cdot \mathrm{min}^{-1}$ in age-matched NA females (144). Thus, $\mathrm{VO}_{2} \max$ is on average $\sim 52 \%$ higher (70 vs. 46 $\left.\mathrm{ml} \cdot \mathrm{kg}^{-1} \cdot \mathrm{min}^{-1}\right)$, in younger ET males compared to age-matched NA males and is $-55 \%$ higher (59 vs. $38 \mathrm{ml} \cdot \mathrm{kg}^{-1} \cdot \mathrm{min}^{-1}$ ), in younger ET females compared to age-matched NA females.

Investigations of male and female masters athletes ( $240 \mathrm{yr}$ and $235 \mathrm{yr}$, respectively) (54, $60,117,122,205,214,226,265,295$ ) show the ability to retain high levels of $\mathrm{VO}_{2} \max$ throughout aging. For example, investigations of older $\mathrm{ET}$ males reveal an average $\mathrm{VO}_{2} \mathrm{max}$ of $48-59 \mathrm{ml} \cdot \mathrm{kg}^{-1} \cdot \mathrm{min}^{-1}$ compared to $26-40 \mathrm{ml} \cdot \mathrm{kg}^{-1} \cdot \mathrm{min}^{-1}$ in age-matched NA individuals (117, $122,205,276)$. While, ET females, ages $40-44,45-49,50-54$, and 55-70 yr exhibited a $\mathrm{VO}_{2} \max$ of $\sim 47, \sim 44, \sim 41$, and $\sim 40 \mathrm{ml} \cdot \mathrm{kg}^{-1} \cdot \mathrm{min}^{-1}$ respectively (295), compared to average values of 34 and $32 \mathrm{ml} \cdot \mathrm{kg}^{-1} \cdot \mathrm{min}^{-1}$ in NA females ages 40-49 and 50-65 yr respectively (144). Stevenson et al (265), reported an average $\mathrm{VO}_{2} \max$ of $\sim 49 \mathrm{ml} \cdot \mathrm{kg}^{-1} \cdot \mathrm{min}^{-1}$ in middle-aged and older (49-67 yr) ET females. Thus, $\mathrm{VO}_{2}$ max in older ET males is on average $~ 64 \%$ higher (54 vs. $33 \mathrm{ml} \cdot \mathrm{kg}^{-1} \cdot \mathrm{min}^{-1}$ ), than in age-matched NA males, and is only $-42 \%$ higher (47 vs. 33 $\mathrm{ml} \cdot \mathrm{kg}^{-1} \cdot \mathrm{min}^{-1}$ ), in older ET females compared to age-matched NA females. Since the $\mathrm{VO}_{2}$ max differences observed between masters females and age-matched NA females are not as large as those observed in masters males $(117,122,205)$, it is possible that the females were not as highly trained as the males. 
Since the training-related differences observed for $\mathrm{VO}_{2}$ max in younger $\mathrm{ET}$ males $(-52 \%$ higher) and females ( $55 \%$ higher) compared to age and gender-matched NA individuals are similar to the corresponding differences observed in older ET males ( $64 \%$ higher) and females ( $-42 \%$ higher), compared to age and gender-matched NA individuals, this suggests that older ET males and females are capable of retaining levels of $\mathrm{VO}_{2}$ max which are similar to their younger ET counterparts.

Mechanisms Responsible for the Larger $\mathrm{VO}_{2} \max$ in ET Compared to $\mathrm{NA}$ Individuals In accordance with the Fick equation $\left(\mathrm{VO}_{2} \max =Q \max \times a-\bar{v} \mathrm{VO}_{2} \max \right)$, where $(\mathrm{Qmax}$ $=\mathrm{SV} \max \times \mathrm{HR \operatorname {max }}), \mathrm{VO}_{2} \max$ may be increased by elevations in $\mathrm{Qmax}$ and/or $\mathrm{a}-\overline{\mathrm{vDO}} \mathrm{O}_{2} \max$. Since HRmax is stable for a given age and does not change with endurance training $(11,83$, $95,117,122,205,222,234,279)$ or decreases slightly $(37,68,257)$ an increased Qmax results from an elevated SVmax. Investigators have observed a larger Qmax resulting from a larger $S V \max$ in younger $(7,11,67,68,123,308,309)$ and older ET males $(82,95,205$, $234,245)$ as well as in younger and older ET females (205) compared to age and gendermatched NA individuals. Furthermore, a larger $\mathrm{a}-\mathrm{v} \mathrm{VO}_{2} \mathrm{max}$ has been observed in younger $(11,240)$ and older ET males $(82,205)$, and in younger and older ET females (205) compared to age and gender-matched NA individuals.

\section{Gender Differences in $\mathrm{VO}_{2} \mathrm{max}$ in $\mathrm{ET}$ Individuals}

When average $\mathrm{VO}_{2}$ max values are compared in younger ET males and females (70 vs 59 $\mathrm{ml} \cdot \mathrm{kg}^{-1} \cdot \mathrm{min}^{-1}$ ) and in older ET males and females (54 vs $47 \mathrm{ml} \cdot \mathrm{kg}^{-1} \cdot \mathrm{min}^{-1}$ ) the $\mathrm{VO}_{2} \mathrm{max}$ in ET males is $-15 \%$ higher. In agreement, Sparling et al. (255) reported a 10-20\% gender 
difference in $\mathrm{VO}_{2} \max \left(\mathrm{ml} \cdot \mathrm{kg}^{-1} \cdot \mathrm{min}^{-1}\right)$ in ET individuals, and Stevenson et al. (265) reported a $13-15 \%$ gender difference $\left(9-10 \mathrm{ml} \cdot \mathrm{kg}^{-1} \cdot \mathrm{min}^{-1}\right.$ lower for females) in both younger and older ET individuals. This suggests that the gender difference in $\mathrm{VO}_{2}$ max in ET individuals is maintained throughout aging.

\section{Mechanisms Responsible for the Larger $\mathrm{VO}_{2} \max$ in ET Males Compared to Females}

In ET individuals, the gender difference in $\mathrm{VO}_{2} \max$ is primarily due to differences in body mass and body composition, [Hb] and thus, arterial oxygen-carrying capacity and delivery, BV, Qmax, and SVmax. No conclusive data exist regarding gender differences in ET individuals in $\mathrm{a}-_{\textrm{DO}} \max$, peripheral oxygen extraction, distribution of blood flow during exercise, respiratory volume, pulmonary diffusion capacity, transit time or ventilationperfusion ratios. The influence of variations in hormone levels during the menstrual cycle, the use of oral contraceptive agents (OCA) and the influence of menopausal status and hormone replacement therapy (HRT) will also be examined.

\section{i) Gender differences in body mass and body composition}

ET females compared to males, tend to have a lower relative LBM (due to both smaller fast-and slow-twitch muscle fibres) $(31,296)$, a higher relative fat mass $(80,296,305)$ and a lower total body mass $(49,255)$. According to Wilmore et al. $(306)$, a younger female distance runner is $\sim 16 \mathrm{~kg}$ lighter in LBM, $\sim 2 \mathrm{~kg}$ heavier in fat mass, $\sim 13 \mathrm{~kg}$ lighter in total body mass, and has an $\sim 6-7 \%$ higher relative body fat percentage than an age-matched male distance runner. Investigations of females revealed fat percentages of $15.3,15.4,16.9$ and $15.2 \%(28,78,305,306)$ for distance runners and $15.4 \%$ for cyclists $(20)$. Since $\mathrm{VO}_{2} \max$ 
$\left(1 \cdot \mathrm{min}^{-1}\right)$ is directly related to the size of the active skeletal muscle mass, a smaller muscle mass, results in a smaller $\mathrm{VO}_{2} \max \left(1 \cdot \mathrm{min}^{-1}\right)$. Additionally, since fat weight does not contribute to energy producticn, a larger fat mass results in a smaller $\mathrm{VO}_{2} \max \left(\mathrm{ml} \cdot \mathrm{kg}^{-1} \cdot \mathrm{min}^{-1}\right)$. Further, a larger fat mass increases the energy cost, and thus the percentage of $\mathrm{VO}_{2}$ max required to perform a given level of submaximal work, in turn, resulting in a lower required workload to reach $\mathrm{NO}_{2} \mathrm{max}$. A meta-analysis showed that in ET younger individuals, the gender difference in $\mathrm{VO}_{2} \max$ was $\sim 40 \%$ in absolute terms, $\sim 20 \%$ when expressed relative to body mass, and was reduced to $\sim 10 \%$ when normalized to LBM (255).

In an attempt to experimentally equalize fat masses in male and female distance runners, Cureton and Sparling (49) added excess weight (7.5\%) to male distance runners. The excess weight decreased the males' $\mathrm{VO}_{2} \max$ by $3.9 \mathrm{ml} \cdot \mathrm{kg}^{-1} \cdot \mathrm{min}^{-1}$ (from 57.8 to $53.9 \mathrm{ml} \cdot \mathrm{kg}^{-1} \cdot \mathrm{min}^{-1}$ ), and eliminated the gender difference in $\mathrm{VO}_{2} \max$. They concluded that all but a very small portion of the gender difference in $\mathrm{VO}_{2} \max \left(1 \cdot \mathrm{min}^{-1}\right)$ is related to body mass differences, if males and females are equally trained.

\section{ii) Gender differences in $[\mathrm{Hb}]$}

Haemoglobin concentration is lower in females compared to males irrespective of age or training status $(7,8)$. For instance, in the U.S., $95 \%$ of females have a $[\mathrm{Hb}]$ between 120 and $160 \mathrm{~g} \cdot \mathrm{l}^{-1}$, while males have a $[\mathrm{Hb}]$ between 140 and $180 \mathrm{~g} \cdot \mathrm{l}^{3}(91)$. Since each gram of haemoglobin can carry $\sim 1.39 \mathrm{ml}$ of oxygen, a lower [Hb] means that less oxygen can be carried by the blood (299). Considering an average $[\mathrm{Hb}]$ of $139 \mathrm{~g} \cdot \mathrm{f}^{-1}$ for females and $158 \mathrm{~g} \cdot \mathrm{l}^{-1}$ for males (106), males have an $\sim 20\left(\mathrm{~g} \cdot \mathrm{l}^{-1}\right)$ larger $[\mathrm{Hb}]$ than females, which results in an $\sim 14 \%$ 
lower arterial oxygen content in females $\left(193 \mathrm{mlO}_{2} \cdot \mathrm{l}^{-1}\right)$ compared to males $\left(220 \mathrm{miO} \cdot \mathrm{l}^{1}\right)$. Similarly, younger and older female marathon runners had a mean $[\mathrm{Hb}]$ of $137 \mathrm{~g} \cdot \mathrm{l}^{-1}$ (297, 280). Thus, a lower [Hb] observed in females may contribute to their lower levels of $\mathrm{VO}_{2}$ max, since less oxygen is available for delivery to the exercising muscles during maximal exercise (229).

In an attempt to determine the proportion of the gender-related difference in $\mathrm{VO}_{2}$ max attributable to differences in $[\mathrm{Hb}]$, Cureton et al. (48) withdrew blood from healthy males to reduce their mean $[\mathrm{Hb}] 14 \%$ (from $153 \mathrm{~g} \cdot \mathrm{l}^{-1}$ to $134 \mathrm{~g}^{-1}$ ), to the same level of healthy females, matched for physical activity level. Before $[\mathrm{Hb}]$ was reduced, $\mathrm{VO}_{2} \max$ was $47 \%\left(1 \cdot \mathrm{min}^{-1}\right)$ and $11.5 \%\left(\mathrm{ml} \cdot \mathrm{kg}^{-1} \cdot \mathrm{min}^{-1}\right)$ larger in the males. By equalizing $[\mathrm{Hb}], \mathrm{VO}_{2} \max$ was $36 \%\left(1 \cdot \mathrm{min}^{-1}\right)$ and $4 \%\left(\mathrm{ml}^{\circ} \mathrm{kg}^{-1} \cdot \mathrm{min}^{-1}\right)$ larger in the males. However, this difference was only $\sim 60 \%$ as large as that predicted if $[\mathrm{Hb}]$ reduction had produced proportional changes in the arterial oxygen content and $\mathrm{a}-\overline{\mathrm{vDO}} \mathrm{O}_{2} \max$. The authors concluded that when males and females are matched for physical activity level, $[\mathrm{Hb}]$ accounts for a significant, but relatively small portion of the gender difference in $\mathrm{VO}_{2} \mathrm{max}$, and that others factors, such as the dimensions of the oxygen transport system and the musculature are of greater importance.

Investigators have observed increases in Qmax following blood withdrawal (91, 141, 142). Thus, individuals with a lower [Hb] may be able to compensate for their smaller oxygen-carrying capacity by delivering more blood to the working muscles to supply the same (or a similar) quantity of oxygen as would individuals with a higher $[\mathrm{Hb}]$. This suggests that in the investigation by Cureton et al. (48), increases in blood flow may have compensated for 
the decrease in oxygen carrying capacity of blood, thus preventing some of the decline in $\mathrm{VO}_{2}$ max that otherwise would have occured.

Robertson et al. (223) examined the effect of induced erythrocythemia on [Hb] and aerobic work capacity in females. Following blood reinfusion which increased [Hb] $16 \%$ (from $127 \mathrm{~g} \cdot \mathrm{l}^{-1}$ to $147 \mathrm{~g} \cdot \mathrm{l}^{-1}$ ), $\mathrm{VO}_{2} \max \left(1 \cdot \mathrm{min}^{-1}\right.$ ) was increased $10 \%$ (from $2.40 \mathrm{l} \cdot \mathrm{min}^{-1}$ to 2.65 $\left.1 \cdot \min ^{-1}\right)$. In a similar experiment of male runners, induced erythrocythemia which produced a $9.3 \%$ increase in $[\mathrm{Hb}]\left(151\right.$ to $165{\mathrm{~g} \cdot \mathrm{I}^{-1}}^{-1}$ was accompanied by a $5.1 \%$ increase in $\mathrm{VO}_{2} \max$ $\left(1 \cdot \mathrm{min}^{-1}\right)\left(5.11 \mathrm{l} \cdot \mathrm{min}^{-1}\right.$ to $\left.5.37 \mathrm{l} \cdot \mathrm{min}^{-1}\right)(106)$. Thus, in comparison to Cureton et al. (48) who withdrew blood from males and concluded that $[\mathrm{Hb}]$ is responsible for only a small portion of the gender difference in $\mathrm{VO}_{2} \max \left(1 \cdot \mathrm{min}^{-1}\right)$, Robertson et al. (223) who induced elevations in [Hb], observed greater changes in $\mathrm{VO}_{2} \max \left(1 \cdot \mathrm{min}^{-1}\right)$, which suggests that [Hb] may be responsible for a larger portion of the gender difference in $\mathrm{VO}_{2} \max \left(1 \cdot \mathrm{min}^{-1}\right)$ than previously observed. For instance, if ET females had their $[\mathrm{Hb}]$ elevated to levels that were equal to ET males, the $\sim 40 \%$ gender difference in $\mathrm{VO}_{2} \max \left(1 \cdot \min ^{-1}\right)$ (255) would be reduced to $\sim 30 \%$. Thus, the lower $[\mathrm{Hb}]$ observed in females, may account for up to $25 \%$ of the gender-related difference in $\mathrm{VO}_{2} \max \left(1 \cdot \mathrm{min}^{-1}\right)$.

\section{iii) Gender differences in $\mathrm{a}-\overline{\mathrm{y}} \mathrm{DQ} \mathrm{Q}_{2} \max$}

Rate of diffusion of oxygen to the muscles depends upon the oxidative capacity and size of the active muscle mass. Although, muscle fibre size is smaller (70-85\%) in female compared to male track athletes, no gender differences have been observed in the relative distribution of muscle fibre types (slow and fast twitch) $(40,41,235)$, in the oxidative enzyme 
activity or in the capillary density (52). Investigators have observed similar values for absolute $\mathrm{a}-\mathrm{v} \mathrm{vO}_{2} \max$ in younger and older ET males compared to age-matched ET fernales $(194,205,321)$. Further, similar values for mixed-venous oxygen content have been reported for active and ET males and females at rest and during maximal exercise $(7,194)$. It was postulated that, although peripheral oxygen extraction is highly dependent upon training state, it is not gender-dependent.

Although investigators have observed similar absolute $\mathrm{a}-\overline{\mathrm{vDO}} \mathrm{O}_{2} \max$ in younger and older ET males compared to age-matched ET females $(194,205,321)$, it is interesting to note that when expressed relative to body mass or LBM, a gender difference exists, such that a$\mathrm{DDO}_{2}$ max is larger in females compared to males. For instance, Ogawa et al. (205) who used the acetylene rebreathe method, observed a $19 \%$ larger a- $-\mathrm{vDO}_{2} \max \left(\mathrm{ml} \mathrm{O}_{2} \cdot \mathrm{kg}^{-1} \cdot \mathrm{dl}^{-1}\right)$ and a $31 \%$ larger a- $\overline{\mathrm{vDO}}{ }_{2} \max \left(\mathrm{ml} \mathrm{O}_{2} \cdot \mathrm{kg} \mathrm{LBM}^{-1} \cdot \mathrm{dl}^{-1}\right)$ in younger ET females compared to agematched ET males. Similarly, they observed a $9 \%$ greater a- $\mathrm{vDO}_{2} \max \left(\mathrm{ml} \mathrm{O}_{2} \cdot \mathrm{kg}^{-1} \cdot \mathrm{dl}^{-1}\right)$ and a $22 \%$ greater a- $-\mathrm{vDO}_{2} \max \left(\mathrm{ml} \mathrm{O}_{2} \cdot \mathrm{kg} \mathrm{LBM}^{-1} \cdot \mathrm{dl}^{-1}\right)$ in older ET females compared to agematched ET males. In agreement, Spina et al. (1993a), who also used the acetylene rebreathe

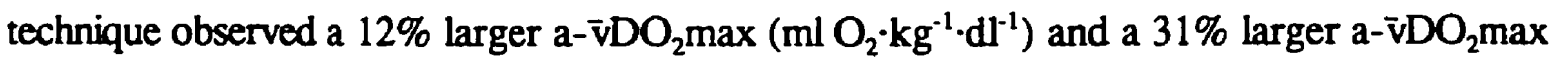
$\left(\mathrm{ml} \mathrm{O}_{2} \cdot \mathrm{kg} \mathrm{LBM}^{-1} \cdot \mathrm{dl}^{-1}\right)$ in older sedentary females compared to age-matched sedentary males, with this difference increasing to a $24 \%$ greater a- $\mathrm{vDO}_{2} \max \left(\mathrm{ml} \mathrm{O}_{2} \cdot \mathrm{kg}^{-1} \cdot \mathrm{dl}^{-1}\right)$ and a $40 \%$ greater a- $\mathrm{vDO}_{2} \max \left(\mathrm{ml} \mathrm{O}_{2} \cdot \mathrm{kg} \mathrm{LBM}^{-1} \cdot \mathrm{dl}^{-1}\right)$ in the older females compared to the older males after an endurance training program. Since neither Ogawa et al. (205) nor Spina et al. (257) analyzed or discussed this data, no reasons were stated for this gender-related difference. 
However, it has been suggested that the potential for oxidative metabolism is higher in females than in males. For instance, females show greater increases in the activity of phosphofructokinase, while males show greater increases in succinate dehydrogenase and citrate synthase activity in response to endurance training $(30,204,253)$. The ratio between the activities of the two enzymes shows that the potential for oxidative metabolism is greater in females than in males (204). It has been postulated that since estrogen enhances the concentration of growth hormone, which in turn increases lypolysis and reduces glycogenolysis (via its supressing action on epinephrine), estrogen may contribute to the greater potential for oxidative metabolism in females (204). Thus, although there is no difference in the absolute $a-\bar{v} \mathrm{DO}_{2}$ max of ET males and females, when expressed relative to body mass and LBM, ET females have an advantage over ET males. Further investigations must be done in this area to uncover the mechanisms responsible for this gender-related difference.

\section{iv) Gender differences in respiratory volumes}

Respiratory volumes are not generally considered a limiting factor for $\mathrm{VO}_{2} \max$ at sea level or at low altitudes. However, due to a female's smaller body size compared to a male, a female's thorax is also smaller. Since thorax size corresponds closely to forced vital capacity (FVC) (which is $\sim 20-25 \%$ smaller in a female) and to residual volume (RV), this results in a smaller total lung capacity $(T L C)$ in a female (TLC $=F V C \times R V)$. At the same submaximal ventilation $\left(\mathrm{V}_{\mathrm{E}}\right)$, a female usually has a smaller tidal volume (TV) and faster respiratory rate $(R R)$ than a male $\left(V_{E}=T V \times R R\right)$. For instance, in elite female runners, 
$\mathrm{V}_{\mathrm{E}} \max$ is $66 \%$ of maximal voluntary ventilation (MVV) $\left(88.8 \mathrm{l} \cdot \mathrm{min}^{-1} / 135 \mathrm{l} \cdot \mathrm{min}^{1}\right)(182)$, while in three elite male runners, values $>90 \%$ of MVV have been observed (183). However, all three of these males had below normal MVV (183). Thus, further investigation is required before conclusions may be drawn regarding the influence of gender differences in respiratory volumes on $\mathrm{VO}_{2} \max$.

v) Gender differences in pulmonary diffusing capacity

Additionally, there is little information concerning gender-related differences in pulmonary diffusion capacity, red blood cell transit times, and ventilation-perfusion ratios during exercise, and no data concerning this difference in ET females. As a result of their smaller body statures, not only do females have lower exercise ventilatory volumes than males, but also smaller pulmonary capacities (189). Thus, due to a reduction in pulmonarycapillary transit time, resulting from smaller pulmonary capillary volumes, ET females who have the ability to generate a large Qmax may be more susceptible to pulmonary diffusion limitations than recently reported in ET males $(216,217)$.

vi) Influence of hormonal variations during the menstrual cycle and the use of OCA

It has been well documented that changes in body fluid balance occur during the menstrual cycle. According to Harrison (119), variations in plasma volume (PV) of such a magnitude are known to affect cardiovascular and thermoregulatory responses during exercise. A normal menstrual cycle lasts from 23 to $35 \mathrm{~d}$ and is comprised of a follicular phase (from the first day of menstruation until ovulation), which may vary in length, and a luteal phase (from ovulation to the onset of the next menstrual period), which lasts for $\sim 14 \mathrm{~d}$. 
During the luteal phase, levels of both estrogen (estradiol $100-350 \mathrm{pg} \cdot \mathrm{ml}^{-1}$ ) and progesterone (4-20 $\mathrm{pg} \cdot \mathrm{mI}^{-1}$ ) are high. Males and females have been shown to have similar PV responses during exercise when females are examined during the follicular phase of their menstrual cycle (96), and thus, most researchers who examine hemodynamic differences between males and females have chosen to test during this phase. However, oligomenorrhea (infrequent menses) and amenorrhea (absent menses) are more prevalent among athletes (10-20\%) compared to the general population (248). Prevalences as high as $40-50 \%$ have been reported in competitive athletes and distance runners $(55,64)$. Approximately one-third of competitive female distance runners (12-45 yr) experience oligomenorrhea or amenorrhea for at least brief periods.

Similar to post-menopausal women, women with amenorrhea display chronically low estrogen levels. Without adequate estrogen levels, amenorrheic athletes lose bone mineral mass (since estrogen facilitates calcium uptake), which increases their susceptibility to fractures and predisposes them to osteoporosis later in life (34). Additionally, a prolonged hypoestrogenic state also deprives amenorrheic athletes of the beneficial effects of estrogen on serum lipoproteins, potentially increasing their risk of coronary artery disease (CAD) (34). The most common and effective treatment for amenorrhea involves the administration of a combined low-dose OCA $\left(0.625 \mathrm{mg} \cdot\right.$ day $\left.^{-1}\right)(248)$, since this provides estrogen to prevent bone loss and premature osteoporosis and progestins to prevent endometrial proliferation (162). Estrogen replacement therapy (ERT) $\left(<50 \mu \mathrm{g}\right.$ estrogen day $\left.^{-1}\right)(248)$ may also be prescribed. The few investigations (with hormonal documentation) in which the effects of 
endogenous and exogenous hormonal variations of a normal menstrual cycle on performance and $\mathrm{VO}_{2}$ max responses of ET females have been examined are inconclusive. DeSouza et al. (55) examined amenorrheic and eumenorrheic runners in the early follicular and mid-luteal phases. The $\mathrm{VO}_{2}$ and $V_{E}$ during submaximal and maximal exercise did not differ with menstrual status or phase. In contrast, Lebrun (162), examined elite female athletes and observed a slight, but significant decreases in $\mathrm{VO}_{2} \max$ in the luteal phase compared to the follicular phase. Schoene et al. (242), observed increases in $V_{E}$ during exercise in the luteal phase in NA females, female middle-and long-distance runners and amenorrheic athletes and a decrease in $\mathrm{VO}_{2}$ max only in NA females. The authors postulated that the lower $\mathrm{VO}_{2} \max$ observed in the luteal phase is due to augmented ventilatory responses to hypoxia and hypercapnea, which occurs during this phase from elevated levels of progesterone (242). Although the resulting increase in $V_{E}$ during exercise did not adversely affect the eumenorrheic ET females in this investigation, the NA females were unable reach as high a level in exercise due to dyspnea. It was suggested that the decreased respiratory drives observed in ET individuals may be an advantage in endurance performance (184).

Similar controversy exists regarding the effects of the administration of a low dose OCA on $\mathrm{VO}_{2} \max$. In active females, Dagget et al. (50) observed a decrease in $\mathrm{VQ}$ max during cycle ergometer exercise in females taking OCA compared to those taking a placebo. Similarly, Notolovitz et al. (202) observed that 6 mo use of a lose-dose OCA in NA females decreased $\mathrm{VO}_{2} \max \left(1 \cdot \mathrm{min}^{-1}\right)$ by $8 \%$ and $\mathrm{VO}_{2} \max \left(\mathrm{ml} \cdot \mathrm{kg}^{-1} \cdot \mathrm{min}^{-1}\right)$ by $7 \%$, despite an increase in body mass. In contrast, Bryner et al. (17) observed that neither the phase of the menstrual 
cycle nor the use of a low dose $\mathrm{OCA}$ altered $\mathrm{VO}_{2} \max$. These investigations suggest that menstrual cycle phase and the administration of $\mathrm{OCA}$ may have a slight influence on $\mathrm{VO}_{2}$ max. However, there is no conclusive evidence stating that exercise performance is significantly affected by menstrual cycle phase or by the use of OCA.

\section{vii) Influence of menopause and HRT}

The average age of menopause for U.S. females is $50 \mathrm{yr}$. Wells et al. (295) examined $\mathrm{VO}_{2} \max$ in relation to age, training status and menstrual status in ET masters females (35-70 yr). When age and training status were controlled, premenopausal, transitional and postmenopausal females had a similar $\mathrm{VO}_{2}$ max. In agreement, Notelovitz et al. (203) found no differences in predicted $\mathrm{VO}_{2}$ max between sedentary age-matched (45-55 yr) pre and postmenopausal females. Similarly, in response to 9 wk of endurance training, Cowan and Gregory (43) observed a $12 \%$ increase in $\mathrm{VO}_{2} \max$ in premenopausal females $(41.3 \pm 4.4)$ and a $19 \%$ increase in postmenopausal females $(56.7 \pm 5.2 \mathrm{yr})$. These researchers concluded that the ability to improve aerobic endurance is independent of menstrual status. Although menopausal status has shown no significant influence on $\mathrm{VO}_{2} \max (265,295)$, another reason for choosing the follicular phase for testing was that postmenopausal females have been shown to display similar levels of estrogen and progesterone as regularly menstruating females who are in this phase (271).

McCole et al. (187) examined the effects of HRT and level of physical activity on $\mathrm{VO}_{2} \max$ in females $(64 \mathrm{yr})$. Although, $\mathrm{V} Q \max$ was significantly greater in ET females compared to both physically active and sedentary females, HRT status was not related to 
$\mathrm{VO}_{2}$ max. Similarly, when Stevenson et al (265) separated their older ET females into a HRT group ( $n=11$ ) and a non-HRT group $(n=19)$, they observed no $\mathrm{VO}_{2} \max$ differences between the two groups. Thus, it appears that menopausal status, with or without HRT does not influence $\mathrm{VO}_{2} \max$.

In summary, the factors that contribute significantly to the gender differences in $\mathrm{VO}_{2} \max$ in ET individuals are body mass and composition, accounting for $\sim 50 \%$, and $[\mathrm{Hb}]$, which accounts for up to $25 \%$. Gender differences in $Q \max , S V \max$ and BV will be reviewed in sections following.

\section{Age-Related Decline in $\mathrm{VO}_{2}$ max}

It has been well documented that $\mathrm{VO}_{2}$ max declines progressively with advancing age regardless of gender or training status $(6,22,54,60,65,66,83,102,117,122,130,132$, $180,205,214,226$ ). Investigators using cycle ergometry and treadmill running in healthy NA individuals of both genders ranging in age from 15 to $80 \mathrm{yr}$, show an $-9-10 \%$ decline in $\mathrm{VO}_{2}$ max per decade, after age 30 yr $(22,65,132)$. However, this age-related decline in $\mathrm{VO}_{2} \max$ may be attenuated by up to $\sim 50 \%$ with endurance training $(117,122,205,226$, 276). For instance, Trappe et al. (276) tested former elite male distance runners after $\sim 22$ yrs $(46.5 \pm 1.6 \mathrm{yr}$ ), and observed decreases of $\sim 6 \%$ per decade in those who continued their training, and decreases of $\sim 15 \%$ per decade in those who became sedentary. In agreement with the latter, the greatest rates of decline in $\mathrm{VO}_{2} \mathrm{max}$ appear to occur in $\mathrm{ET}$ individuals who stop or greatly decrease their training $(54,60)$. Similar age-related decreases in $\mathrm{VO}_{2}$ max have been reported for ET females (25-55 yr) $(65,205,282,295)$. For instance, Wells et al. (295) 
reported a $0.58 \mathrm{ml} \cdot \mathrm{kg}^{-1} \cdot \mathrm{min}^{-1} \cdot \mathrm{yr}^{-1}$ decline in $\mathrm{VO}_{2} \max$ in female masters runners $(35-70 \mathrm{yr})$. Further, Vaccaro et al. (282) observed decreases of $7 \%$ per decade in $\mathrm{VO}_{2}$ max in female swimmers (20-70 yr), with a significant decline occuring after age $40 \mathrm{yr}$.

\section{Mechanisms Responsible for the Age-Related Decline in $\mathbf{V O}_{2} \mathbf{m a x}$}

There is considerable controversy regarding the mechanisms responsible for the agerelated decline in $\mathrm{VO}_{2}$ max in both NA and ET individuals. Differences in body mass and body composition, Qmax, SVmax, $\mathrm{HR}_{\max }$ and $\mathrm{a}-\overline{\mathrm{v}} \mathrm{VO}_{2} \max$ are all possible contributing factors. i) Age-related changes in body mass and composition and other non-cardiac factors

When compared to younger ET males and females, older ET males and females tend to have a lower relative $\operatorname{LBM}(83,218,214)$ or a maintenance of $\operatorname{LBM}(122,180)$, a higher relative fat mass $(62,122,214,276)$ and a higher total body mass (which may level off at 50 $60 \mathrm{yr}$ and then decline) $(62,122,214,276)$. An increase in fat mass, which results in an increase in body mass, results in a decrease in $\mathrm{VO}_{2} \max \left(\mathrm{ml} \cdot \mathrm{kg}^{-1} \cdot \mathrm{min}^{-1}\right)(83,205)$. In addition, since the absolute levels of training intensity and/or volumes tend to decrease with age in ET males and females $(69,214,226)$, this may also result in a decrease in $\mathrm{VO}_{2}$ max. For instance, the overall training volumes of masters athletes was shown to be only $\sim 50-60 \%$ as great as that of younger ET individuals (222). An increased prevalence of injuries or decreased levels of motivation may contribute to the reduction in training (138). Additionally, other noncardiac factors, such as: orthopaedic limitations, reductions in strength, reductions in the threshold for neuromuscular fatigue and a decreased ability of NA individuals to attain a true $\mathrm{VO}_{2} \max$, may modify the rate of decline in $\mathrm{VO}_{2} \max$ independent of age (22). 


\section{ii) Age-related changes in the Fick determinants of $\mathrm{VQ}_{2} \max$}

Regarding the "Fick" determinants of $\mathrm{VO}_{2} \max (\mathrm{HR} \max , \mathrm{SVmax}, \mathrm{Qmax}$ and a$\left.\mathrm{vDO}_{2} \max \right)$, one consistent finding is an age-related decline in $\mathrm{HR} \max (66,83,115,117,122$, $205,222,234)$, which in turn results in a reduction in $Q \max$ and thus $\mathrm{VO}_{2} \max (117,205$, 222). Saltin et al (234) found similar reductions in HRmax in both sedentary and well trained active orienteers (50-70 yr) (ie., from 175-180 to $\sim 160 \mathrm{bpm}$ ). In agreement, Pollock et al. (214), observed similar age-related decreases in HRmax in masters athletes whose activity levels decreased over time compared to those who continued training. Some investigators examining older ET males and females compared to their younger ET counterparts suggest that the decline in $\mathrm{VO}_{2} \max$ is due solely to this age-related decline in $\operatorname{HRmax}(117,122)$, while other researchers show a contribution of only $25-40 \%$ from this mechanism $(205,222)$. The roles of $\mathrm{Qmax}, \mathrm{SV} \max$ and a- $\mathrm{vDO} \mathrm{V}_{2} \max$ in explaining the age-related decline in $\mathrm{VO}_{2} \max$ in males and females are controversial $(81,100,117,122,205,222,224,318)$ and will be reviewed in sections following.

Mechanisms Responsible for the Attenuated Age-Related Decline in $\mathrm{VO}_{2} \max$ in ET Compared to NA Individuals

Since masters athletes are better able to maintain LBM and tend to have a smaller amount of subcutaneous fat than NA age-matched individuals (122,180,214), this results in a larger $\mathrm{VO}_{2} \max$ in both $1 \cdot \mathrm{min}^{-1}$ and $\mathrm{ml} \cdot \mathrm{kg}^{-1} \cdot \mathrm{min}^{-1}$. In addition, some investigators have shown smaller age-related reductions in $\mathrm{HR} \max (205), \mathrm{a}-\mathrm{vDO}_{2} \max (205,117,122)$ and $\mathrm{SVmax}$ $(117,122)$ in ET, compared to NA individuals. For instance, while HRmax decreased by 
$13 \%$ in sedentary individuals, it decreased by only $7 \%$ in ET individuals with age (205). Similarly, while a- $\mathrm{vDO}_{2}$ max decreased by $12 \%$ in sedentary individuals, it decreased by only $4 \%$ in ET individuals with age (205). In comparison, Heath et al. (122) and Hagberg et al. (117), observed similar high levels of $\mathrm{SVmax}$ and $\mathrm{a}-\overline{\mathrm{vDO}}{ }_{2} \mathrm{max}$ in ET young and older males, compared to sedentary older males. These authors postulated that the maintenance of a chronic level of endurance training can delay the age-related decline in SVmax and a$\mathrm{vDO}_{2} \max$, thus, attenuating the age-related decline in $\mathrm{VO}_{2} \max$.

\section{Summary}

ET individuals have a larger $\mathrm{VO}_{2} \max$, due to a larger $\mathrm{Qmax}, \mathrm{SV} \max$ and a- $\mathrm{vDO} \mathrm{D}_{2} \max$ compared to age and gender-matched NA individuals. Additionally, ET males have a larger $\mathrm{VO}_{2} \max$ than ET females regardless of age, which is primarily due to gender differences in body size and composition, [Hb], Qmax, SVmax and BV. Further, although $\mathrm{VO}_{2} \max$ declines progressively with age regardless of gender or training status, this age-related decline is attenuated in ET individuals.

\section{Cardiac Output and Stroke Volume in ET Individuals and the Relationship}

\section{to Gender and Age}

\section{Maximal Cardiac Output in ET Compared to NA Individuals}

The rate at which the heart pumps blood in to the circulatory system is a limiting factor for aerobic capacity. Investigators have shown that resting and exercise $Q$ is significantly larger in younger $(7,11,67)$ and older ET males $(82,117,122,205,222,245)$ compared to age-matched NA males. Similarly, a larger $Q \max$ has been observed in younger 
and older ET females (205) compared to NA age-matched females. For example, Ogawa et al. (205) reported that in younger ET males (21-31 yr), Qmax was $29 \%$ greater (27.4 $1 \cdot \mathrm{min}^{-1}$ vs $\left.21.21 \cdot \mathrm{min}^{-1}\right)$ and likewise, in older ET males (59-72 yr), Qmax was $26 \%$ larger $\left(20.51 \cdot \mathrm{min}^{-1}\right.$ vs $\left.16.31 \mathrm{~min}^{-1}\right)$ than in NA age-matched males. On the other hand, in younger (18-30 $\left.\mathrm{yr}\right)$ and older ET females (51-72 yr), Qmax was $21 \%$ larger $\left(18.41 \cdot \mathrm{min}^{-1}\right.$ vs $\left.15.21 \cdot \mathrm{min}^{-1}\right)$ and $20 \%$ larger $\left(14.31 \cdot \mathrm{min}^{-1}\right.$ vs $\left.11.91 \cdot \mathrm{min}^{-1}\right)$, respectively, compared to NA age-matched females. In agreement, during supine exercise, with cardiac blood pool imaging and echocardiography, Seals et al. (245) observed a $14 \%$ larger peak CI in masters males (64 $\pm 2 \mathrm{yr}$ ) compared to age-matched NA males. Similarly, during treadmill running, using radionuclide angiography, Fleg et al (82) revealed a $22 \%$ larger peak $\mathrm{CI}$ in masters males (63 $\pm 7 \mathrm{yr}$ ) compared to agematched NA males. Increases in $Q$ may result from increases in SV and/or increases in HR, since $Q=S V \times H R$.

\section{Maximal Stroke Volume in ET Compared to NA Individuals}

As a result of endurance training, bradycardia (enhanced vagal tone) is observed at rest $(94,98,221,229)$, a decrease or no change in $\mathrm{HR}$ is observed during submaximal exercise $(11,83,117,122,131,205,222,279)$ and a slight decrease may be observed in HRmax $(37,257)$,thus, an increased $Q$ results from an elevated SV. Further, numerous investigators have observed a significantly larger SV at rest, and during submaximal and maximal exercise in younger $(7,9,11,46,67)$ and older ET males $(82,117,122,205,222$, 245) as well as in younger and older ET females (205) compared to NA age and gendermatched individuals. For instance, Ogawa et al. (205) reported that SVmax was 34\% greater 
in younger ET males (21-31 yr) compared to NA age-matched males (154 ml vs $115 \mathrm{ml}$ ), and likewise, 23\% larger in older ET males (59-72 yr) compared to NA age-matched males (124 $\mathrm{ml}$ vs $101 \mathrm{ml})$. In younger ET females (18-30 yr), SVmax was $28 \%$ higher than in NA agematched females (102 $\mathrm{ml}$ vs $80 \mathrm{ml}$ ), whereas, it was $15 \%$ higher in older ET females (51-72 yr), compared to NA age-matched females ( $85 \mathrm{ml}$ vs $74 \mathrm{ml}$ ). Similarly, Seals et al. (245) observed a $19 \%$ larger peak SVI in masters males (64 $\pm 2 \mathrm{yr}$ ) compared to NA age-matched males. In agreement, Fleg et al (82) observed that the $22 \%$ larger peak CI was due entirely to a higher peak SVI in masters males compared to NA age-matched males, while HRmax did not differ between groups.

Mechanisms Responsible for the Larger SVmax in ET Compared to NA Individuals

Stroke volume is affected by hemodynamic and myocardial factors. Since SV = LVEDV - LVESV, an enharced SV may rcsult from an increased LVEDV and/or a decreased LVESV.

i) LVEDV

A larger LVEDV has been observed in younger $(46,104,170)$ and older ET males $(82,245)$ as well as in younger ET females $(32,98,200,231,319)$ compared to NA age and gender-matched individuals. For instance, Seals et al. (245) observed a larger LVEDV in masters males compared to NA age-matched males, and Fleg et al. (82) observed an $11 \%$ larger LVEDV in masters males compared to NA age-matched males.

The only available studies in which LVEDV was examined in older females are longitudinal. In these investigations, previously sedentary females $(63 \pm 4 \mathrm{yr})$ became 
endurance trained over a period of $9-12 \mathrm{mo}\left(4-5 \mathrm{~d} \cdot \mathrm{wk}^{-1}, 45 \mathrm{~min}-1 \mathrm{hr} \cdot \mathrm{d}^{-1}, 60-85 \%\right.$ of $\left.\mathrm{VO}_{2} \mathrm{max}\right)$ $(257,258)$. Although an increased $\mathrm{VO}_{2} \max$ was observed, no increases were detected in Qmax, SVmax or LVEDV. Additionally, no differences were found for left ventricular enddiastolic diameter (LVEDD), LV mass or LVESV (258). The entire training-induced elevation in $\mathrm{VO}_{2} \max$ was due to an increase in $\mathrm{a}-\mathrm{DDO}_{2} \max$. It was postulated that since older males (66) and younger females display increases in Qmax, SVmax, LVEDV and LVEDD in response to short-term training programs $(47,147,150,259)$ and that since the postmenopausal females in the investigations by Spina et al. $(257,258)$ were not using HRT, the lack of central adaptations may be due to the deficiency of circulating estrogen after menopause $(114,210,238,239)$. For instance Schaible et al. (238) observed that although an identical running program in male and female rats produced equal increases in skeletal muscle oxidative capacity, cardiac function adaptations were elicited only in the male rats. In agreement, Green et al. (114) examined postmenopausal females who had engaged in regular endurance training at least 3 times per week for more than $1 \mathrm{yr}$. A significantly larger Qmax and SVmax were observed in the females who had been taking ERT for at least 1 yr compared to those who had never used ERT.

In contrast, Morrison et al. (199) observed an increase in resting LVEDD in previously sedentary postmenopausal females $(51 \mathrm{yr})$ who became endurance trained over 8 mo (3 times $\cdot \mathrm{wk}^{-1}, 40 \mathrm{~min} \cdot \mathrm{d}^{-1}, 65-75 \%$ of hear rate reserve). In agreement, Schocken et al. (241) observed an increase in LVEDV and SV during exercise at the same absolute cycling intensity following $12 \mathrm{w}$ of endurance training ( 3 times $\cdot \mathrm{wk}^{-1}, 30 \mathrm{~min} \cdot \mathrm{d}^{-1}, 70-85 \%$ of peak HR), 
in 24 previously sedentary elderly individuals $(72 \mathrm{yr}), 18$ of whom were female. However, there was no indication of HRT status in these latter two investigations. Thus, it is premature to conclude whether older females display similar training-induced adaptations in LVEDV as observed previously in ET males and younger ET females.

\section{ii) LVESY}

Additionally, a smaller LVESV has been observed in younger and older ET males (62, $82,245)$ compared to NA age-matched males. For instance, Seals et al. (245) observed that in addition to the increase in LVEDV, masters males also displayed a greater exercise induced elevation in ejection fraction (EF), and a larger decrease in ESV, compared to NA agematched males. In agreement, Fleg et al. (82) observed that the larger SVI in masters males, compared to NA age-matched males, was accompanied by a 7\% higher EF. However, there are no cross-sectional data available which examine LVESV in ET females, and thus it is unknown whether ET females exhibit similar adaptations as those observed in ET males.

iii) Mechanisms responsible for the enhanced LVEDV and LVESV in ET compared to

\section{NA individuals}

In ET males and younger ET females, an increased LVEDV is attributed to an increased preload, which in turn results from an enhanced venous return $(145,227,309)$ and an augmented BV $(35,45,220)$. An increased LVEDV may also be due to increased cardiac dimensions $(104,131,198,209,279)$ and improved compliance of the LV cavity (312). A decreased LVESV is attributed to enhanced myocardial contractility $(3,260)$ and decreased afterload $(5,145,227,309)$. 
Preload. Endurance trianed individuals have an elevated SV due to an increased preload $(145,227,309)$. According to Frank-Starling's law, an increased preload results in greater stretch on the myocardial fibres which leads to a more forceful ventricular contraction (141), a larger volume of ejected blood, and thus an elevated SV $(141,142,165)$. Preload is affected by venous return, which is influenced by body position, skeletal muscle pump, respiratory pump, venous tone and BV.

Upright exercise results in a decreased venous return compared to supine exercise, due to a gravitational venous pooling in the lower extremities $(9,212)$. Thus, caution must be taken when comparing LVEDV from investigations which examine participants in different body positions. During exercise, the active skeletal muscles contract and compress the veins, thereby facilitating venous return $(5,304)$. In addition, as respiration increases, specifically inspiration, intrathoracic pressure decreases and compresses the thoracic veins, thereby facilitating venous return (304). Additionally, as venous sympathetic tone increases, the diameter and compliance of the veins decrease, thereby increasing venous pressure and flow to the right atrium. Concurrently, as sympathetic vasoconstriction of the inactive arteries and arterioles increases, blood will be redistributed to the active skeletal muscles, facilitating venous return (304). Lastly, an augmented BV will enhance venous return and right atrial filling pressure by creating a larger pressure gradient from the central venous reservoir to the right atrium (36).

Cardiac Dimensions. It has been postulated that endurance training results in an increased LVEDD due to an elevated SV which places a chronic volume overload on the 
heart $(99,198)$. According to Laplace's Law, a chronic volume overload is accompanied by an increased end-diastolic wall stress, which the heart compensates for by increasing its wall thickness $(11,99)$. An increased LV cavity is better able to cope efficiently with an increased preload, which in turn will lead to enhanced diastolic filling and thus, a larger SV $(11,70,99)$. Using echocardiography, investigators have observed larger LVEDD in ET younger $(198,279)$ and older males $(117,122,245)$, compared to NA age-matched males. Similarly, higher than average LVEDD have been observed in younger female field hockey players (319), ballet dancers (32), softball players (231), cyclists, long distance runners and skiers $(71,98,200,221)$ compared to age-matched NA females. Additionally, investigators have observed a thicker interventricular septum or posterior wall (which serves to normalize wall stress) with an unchanged wall thickness to radius ratio (volume overload hypertrophy) in younger ET males $(70,198)$, younger ET females $(71,200,231)$ and older ET males (122, 245) compared to NA age and gender-matched individuals. However, an increased LVEDD does not appear to be required for an increased $\mathrm{SV}$ and thus $\mathrm{Q}$ and $\mathrm{VO}_{2} \mathrm{max}$ to occur, since improvements in these variables have been observed after short-term training programs without an increase in LVEDD in younger $(68,82,310)$, and older males $(66)$, and in younger fermales (307).

Myocardial Contractility. Endurance trained individuals have an increased SV due to enhanced myocardial contractility $(104,123,211,245)$. Endurance training has been shown to result in an improvement in myocardial contractility in response to $\beta$-adrenergic receptor ( $\beta$-AR) stimulation with isoproterenol in younger healthy males and females ( $27 \pm$ 
4 yr) (260). Similarly, younger ET males showed an enhanced inotropic response to $\beta$-AR stimulation with dobutamine (after parasympathetic blockade with atropine), compared to age-matched NA males (129). In agreement, Ehsani et al (66) observed enhanced myocardial contractility in response to $1 \mathrm{yr}$ of endurance training $\left(60-80 \%\right.$ of $\left.\mathrm{VO}_{2} \max \right)$ in older males (64 \pm 3 yr) (66). The augmented SVmax resulted from an enhanced EF (71-78\% improved) and a shift in the ESV to SBP ratio to the left, suggestive of an enhanced inotropic state.

An increased myocardial contraction may result from an increased amount of circulating catecholamines, or an enhanced response to the catecholamines $(104,278)$. It has been postulated that myocardial contractility plays a key role in LV performance during maximal exercise, since catecholamines have been shown to increase greatly after $50 \%$ of $\mathrm{VO}_{2} \max (108,121)$.

Afterload. Endurance trained individuals have an augmented SV due to a decreased afterload, which is evident in their lower BP responses during exercise, compared to NA individuals $(145,181,234)$. The major site of resistance to blood flow is in the arterioles (11) and during exercise, arteriolar vasodilation occurs via neural, local metabolic, physical and hormonal factors. A decreased TPR has been observed in ET individuals $(82,105,128,152$, 181,309 , which results in a decreased afterload, and thus, an enhanced blood flow and SV (11). For instance, Fleg et al. (82) found greater decreases in systemic vascular resistance from rest to peak exercise, as well as at peak exercise in older ET males compared to agematched NA males, suggesting decreased afterload may play a role in increasing SV. Although a reduced afterload may facilitate systolic emptying, it is not always 
observed after endurance training and thus, is not essential for an increased SV and thus $Q$ to occur $(68,259)$. For instance, Spina et al. (259) observed an increase in SVmax and Qmax, without a significant change in MAPmax or TPRmax after a 12 wk training program in younger males and females.

\section{iv) Mechanisms of primary importance}

As previously reviewed, it appears that neither an increased LVEDD $(66,307,308$, $310)$ or a decreased afterload $(259,309)$ are required for an increased SVmax and Qmax to occur. It is generally accepted from observations of healthy males that the exercise-induced rise in SV results from a combination of enhanced myocardial contractility (enhanced systolic emptying) and elevated preload (enhanced diastolic filling) $(82,123,211,212,220,245)$. However, controversy exists regarding which one of these mechanisms is the primary mechanism responsible for augmenting SV throughout incremental exercise to maximum.

Several investigators have postulated that the Frank-Starling mechanism has its greatest influence at low to moderate work rates while myocardial contractility has its greatest influence at high work rates $(7,104,108,123,211,268)$. These investigators and others observed that SV increased until it reached a plateau at exercise intensities between 40 and $60 \%$ of $\mathrm{VO}_{2} \max (7,104,108,123,211,268)$. For example, Plotnick et al. (211) used gated radionuclide angiography and observed an increase in LVEDV and SV with no change in LVESV during low exercise intensities on a cycle ergometer in moderately active males (26$50 \mathrm{yr}$ ). At moderate exercise intensities LVEDV plateaued while LVESV decreased. At maximum exercise LVEDV decreased to resting levels, SV plateaued, while LVESV 
decreased further. The authors postulated that in moderately active individuals, the FrankStarling mechanism is primarily responsible for increasing SV and $Q$ at low to moderate exercise intensities.

During progressive exercise to maximum, Ginzton et al. (104) used 2-D echocardiography to examine male and female competitive long-distance runners (19 $\pm 1 \mathrm{yr})$ and NA age and gender-matched individuals. Although LVEDV in the ET individuals was significantly higher at all exercise intensities, similar increases in LVEDV were observed at low exercise intensities and similar progressive decreses were seen in LVEDV throughout the remainder of exercise in both groups. At higher HR (> $170 \mathrm{bpm}$ ) the LVEDV and SV of both groups decreased progressively. The investigators postulated that although ET individuals have a larger LVEDV than NA individuals, myocardial contractility has a greater effect on increasing SVmax, than does the Frank-Starling mechanism, regardless of training status.

In healthy sedentary males (20-50 yr) Higginbotham et al. (123) used gated radionuclide angiography and therodilution and observed that at low exercise intensities, SV increased primarily by increases in LV filling pressure and LVEDV with only slight decreases in LVESV. While at high exercise intensities, despite increases in filling pressure, LVEDV decreased. However, SV was maintained due to progressive decreases in LVESV. Thus, it was speculated that at high exercise intensities (HR > $170 \mathrm{bpm})$, a shortened DT, would limit ventricular filling, LVEDV and thus, SV would plateau (104, 123, 211). Any further increases in $Q$ beyond $40-60 \%$ of $\mathrm{VO}_{2} \max$ would be due to increases in $\mathrm{HR}$ and thus, enhanced contractility (104, 108, 123, 211). 
However, in challenge to this view, more recent investigators have shown that ET males have the ability to use the Frank-Starling mechanism effectively up to $\mathrm{VO}_{2} \max (46$, $105,128,152,165,220,230,289)$. These investigators observed a progressive increase in SV throughout incremental exercise to maximum without a plateau in ET males $(46,67,68$, $105,152,220,222,285,289$ ). For instance, Rerych et al. (220), measured LV volumes using radionuclide angiography in male and female college athletes, before and after 6 mo of swim training. They found that endurance training resulted in an enhanced EDV, Q and SV, while $\mathrm{HR}$ and $\mathrm{EF}$ were maintained. The authors postulated that the increased Qmax was due to an enhanced ability of the LV to dilate from rest to exercise and to eject fully the increased EDV at the same HR. In agreement, Rubal et al. (230) also used radionuclide angiography and observed that although both younger pentathletes and NA age-matched males relied on similar mechanisms to improve cardiac function during exercise, the pentathletes had a larger EDV and SV at each exercise stage. These authors concluded that since EF did not differ when HR was controlled, the pentathletes achieved an enhanced SV by augmenting LV filling via the Frank-Starling mechanism. Similarly, Crawford et al. (46), used 2-D echocardiography and observed an increased EDV and SV at rest and throughout moderate intensity exercise in both competitive and non-competitive male marathon runners. Although EDV and SV reached a plateau at $70 \%$ of peak HR in the non-competitive males, it continued to increase up to peak exercise in the competitive males. The authors proposed that the competitive males made greater use of the Frank-Starling mechanism during strenuous exercise than the noncompetitive males. 
More recently, some investigators have used the acetylene rebreathe method to measure $Q(105,152,222,289)$. For instance, Gledhill et al. (105) and Krip et al. (152) examined cardiac function in ET compared to NA males. Although they observed a plateau in SV at $\sim 40 \%$ of $\mathrm{VO}_{2} \max$ in the NA males, the SV of the ET males increased progressively, throughout incremental exercise to maximum, without a plateau. Similarly, Rivera et al. (222) observed that SV did not reach a plateau, but continued to increase up to maximum in 5 out of 11 masters males. Providing further support for this finding, Warburton et al. (289) observed a progressive increase in SV up to maximum, without a plateau in 9 elite male cyclists. The progressive increase in SV to maximum observed in the ET males was associated with an enhanced DFR (and thus an enhanced preload) and LVER (enhanced myocardial contractility and/or decreased afterload), with the primary advantage being in the DFR $(33,71,105,152,157,186,289)$. It was postulated that the ability to use the FrankStarling mechanism throughout incremental exercise to maximum, is due to an elevated BV in ET males $(35,105,152,220,289)$. Thus, it appears that chronic endurance training results in cardiac function adaptations, such that SV continues to increase throughout incremental exercise to maximum without a plateau.

In summary, regardless of age or gender, ET compared to NA individuals have a larger Qmax, due to an elevated $\mathrm{SVmax}$, which in turn, leads to a greater $\mathrm{VO}_{2} \max$. The elevated SVmax is due primarily to an enhanced DFR, which results from a larger BV in ET compared to NA males. Recent investigators have shown that in contrast to NA males, the SV of ET males does not plateau at submaximal intensities, but continues to increase 
throughout incremental exercise to maximum.

\section{Gender Differences in Qmax and SVmax in ET Individuals}

Although ET females display a larger Qmax and SVmax than NA females, higher Qmax values are observed in ET males compared to ET females $(7,205)$. For example, Ogawa et al (205) reported that in younger ET females (18-31 yr), Qmax was 33\% lower (18.4 $\mathrm{l} \cdot \mathrm{min}^{-1}$ vs $\left.27.4 \mathrm{l} \cdot \mathrm{min}^{-1}\right)$ than in age-matched ET males. Similarly, in older ET females (51-63 yr), Qmax was $31 \%$ lower $\left(14.31 \cdot \mathrm{min}^{-1}\right.$ vs $\left.20.51 \cdot \mathrm{min}^{-1}\right)$ than in ET males $(59-72 \mathrm{yr})$. Since HRmax is not gender related $(7,205,318)$, the smaller Qmax observed in ET females is due to their smaller SVmax. Moreover, Ogawa et al. (205) reported that in younger ET females (21-31 yr) SVmax was 34\% lower (102 $\mathrm{ml}$ vs $154 \mathrm{ml}$ ) than in age-matched ET males. Similarly, in older ET females (51-63 yr) SVmax was $30 \%$ lower ( $85 \mathrm{ml}$ vs $124 \mathrm{ml}$ ) than in ET males (59-72 yr). In contrast, Mier et al. (192) observed a $10 \%$ increase in SV after 10 d of cycle ergometer training in both males $(29 \pm 2 \mathrm{yr})$ and females $(25 \pm 2 \mathrm{yr})$. The authors postulated that males and females have similar cardiac reserve capacities for increasing SV as a result of endurance training.

Regarding submaximal exercise, previous researchers have observed differences in the magnitude of $Q$ and $S V$ responses in males and females $(7,8,92,321)$. For example, in a classic study by Astrand et al. (72), at a $\mathrm{VO}_{2}$ of $1.5 \mathrm{l} \cdot \mathrm{min}^{-1}$, healthy NA females ( $21 \mathrm{yr}$ ) had a significantly higher HR (45 bpm or $30 \%$ ) and Q, and a smaller SV than NA age-matched males. Similarly, Becklake et al. (8) reported that at any given submaximal workload on a cycle ergometer, sedentary females (20-39 yr) had a higher $H R$, and $Q$ than age-matched 
sedentary males, although no gender difference was observed in SV. It was postulated that at the same absolute workload, the higher HR of females, reflects either a higher relative exercise intensity and/or a compensation for their lower SV compared to males $(125,318)$. Additionally, it was postulated that the higher $Q$ observed at low exercise intensities in females was a compensation for their lower $[\mathrm{Hb}]$ compared to males $(7,8,91)$.

In contrast, Zwiren et al (321) compared male and female runners and swimmers who were matched for $\mathrm{VO}_{2} \max \left(62.3\right.$ and $60.3 \mathrm{ml} \cdot \mathrm{kg} \mathrm{LBM}^{-1} \cdot \mathrm{min}^{-1}$, respectively). At a $\mathrm{VO}_{2}$ of 1.5 $1 \cdot \min ^{-1}$, there were no gender differences in $\mathrm{Q}$, although the females had a lower $\mathrm{SV}$ and a higher HR (23 bpm or 18\%). Zwiren et al. (321) postulated that although the pattern of gender differences in the responses to exercise are similar to the earlier investigations $(7,8)$, the magnitude of the gender differences are smaller, which may indicate a gender difference in physical activity level in the earlier studies.

Although the ratio of $\mathrm{Q}$ to $\mathrm{VO}_{2}$ during submaximal work has been reported to be higher in females than males $(5,64)$, investigators reported that there are no gender differences in the $\mathrm{QVO}_{2}$ slope during exercise $(188,321)$. For instance, McKelvaney et al. (188) reported a $\mathrm{Q}-\mathrm{VO}_{2}$ slope of $5.1 \mathrm{l} \cdot \mathrm{min}^{-1}$ in males and $4.6 \mathrm{l} \cdot \mathrm{min}^{-1}$ in females $(66 \pm 6 \mathrm{yr})$. These are similar to the $5-61 \cdot \mathrm{min}^{-1}$ slopes reported for younger (77) and older males (275). This suggests that the change in $\mathrm{Q}$ for a given $\mathrm{VO}_{2}$ during exercise, is not influenced by age or gender.

Mechanisms Responsible for the Larger Qmax and SVmax in ET Males vs ET Females In ET individuals, the gender difference in $\mathrm{Qmax}$ and $\mathrm{SV} \max$ are due to differences 
39

in body mass and composition, heart size, and the mechanisms responsible for augmenting SV during exercise.

i) Gender differences in body mass and body composition

In an investigation by Ogawa et al. (205), the 33\% lower $Q \max \left(1 \cdot \mathrm{min}^{-1}\right)$ reported for ET females compared to age-matched ET males (21-31 yr) was reduced to $17 \%$ when normalized to body mass $\left(332 \mathrm{ml} \cdot \mathrm{kg}^{-1} \cdot \mathrm{min}^{-1}\right.$ vs $\left.401 \mathrm{ml} \cdot \mathrm{kg}^{-1} \cdot \mathrm{min}^{-1}\right)$ and further reduced to $9 \%$ when expressed relative to $\mathrm{LBM}\left(403 \mathrm{ml} \cdot \mathrm{kg} \mathrm{LBM}^{-1} \cdot \mathrm{min}^{-1}\right.$ vs $435 \mathrm{ml} \cdot \mathrm{kg} \mathrm{LBM} \cdot \mathrm{m}^{\text {inh }}$ ). Similarly, the $31 \%$ lower Qmax $\left(1 \cdot \mathrm{min}^{-1}\right)$ reported for ET females (51-63 yr) compared to ET males (59-72 $\mathrm{yr})$ was reduced to $22 \%$ when normalized to body mass $\left(242 \mathrm{ml} \cdot \mathrm{kg}^{-1} \cdot \mathrm{min}^{-1}\right.$ vs $\left.312 \mathrm{ml} \cdot \mathrm{kg}^{-1} \cdot \mathrm{min}^{-1}\right)$ and further reduced to $14 \%$ when expressed relative to LBM (326 ml $\cdot \mathrm{kg}$ $\mathrm{LBM}^{-1} \cdot \mathrm{min}^{-1}$ vs $\left.380 \mathrm{ml} \cdot \mathrm{kg} \mathrm{LBM}^{-1} \cdot \mathrm{min}^{-1}\right)$. Additionally, the $34 \%$ lower $\mathrm{SVmax}(\mathrm{ml})$ reported for ET females compared to age-matched ET males (21-31 yr) was reduced to $18 \%$ when normalized to body mass $\left(1.85 \mathrm{ml} \cdot \mathrm{kg}^{-1}\right.$ vs $\left.2.25 \mathrm{ml} \cdot \mathrm{kg}^{-1}\right)$ and further reduced to $10 \%$ when expressed relative to LBM $\left(2.25 \mathrm{ml} \cdot \mathrm{kg} \mathrm{LBM}^{-1}\right.$ vs $\left.2.50 \mathrm{ml} \cdot \mathrm{kg} \mathrm{LBM}^{1}\right)$. Likewise, the $30 \%$ lower SVmax (ml) reported for ET females (51-63 yr) compared to ET males (59-72 yr) was reduced to $22 \%$ when normalized to body mass $\left(1.45 \mathrm{ml} \cdot \mathrm{kg}^{-1}\right.$ vs $\left.1.87 \mathrm{ml} \cdot \mathrm{kg}^{-1}\right)$ and further reduced to $14 \%$ when expressed relative to $\mathrm{LBM}\left(1.95 \mathrm{ml} \cdot \mathrm{kg} \mathrm{LBM}^{1}\right.$ vs $\left.2.27 \mathrm{ml} \cdot \mathrm{kg} \mathrm{LBM}^{-1}\right)$. This suggests that up to two-thirds of the gender difference in Qmax and SVmax in younger ET individuals and one-half of the gender difference in these two variables in older ET individuals, can be accounted for by differences in LBM. 


\section{ii) Gender differences in heart size}

Echocardiographic investigations in females reveal significantly smaller absolute cardiac dimensions and wall thicknesses than males $(109,200)$. For example, Fagard et al. examined male (73) and female (72) long-distance runners and observed LV internal diameters of $53 \mathrm{~mm}$ and $46 \mathrm{~mm}$ and LV masses of $122 \mathrm{~g} / \mathrm{m}^{2}$ and $108 \mathrm{~g} / \mathrm{m}^{2}$, in the males and females, respectively. Using nuclear magnetic resonance imagery, some investigators observed smaller LV masses in ET females compared to ET males, even when correction was made for $\operatorname{LBM}(98,221)$. In contrast, Devereux et al. (57) used creatine excretion as a correction for LBM and eliminated the gender difference in LV mass index. Astrand et al. (72) reported that in healthy NA younger females ( $21 \mathrm{yr}$ ), heart volume, relative to body weight and body surface area is $\sim 80-90 \%$ of a male's (up until about age 60 ). Astrand et al. (72) postulated that a smaller heart (in both absolute and relative terms) in females may lead to a smaller SVmax due to a reduced LV filling capacity, which would contribute to a smaller Qmax and thus, $\mathrm{VO}_{2} \max$. However, no gender differences were observed in $\mathrm{LV}$ dimensions in ET males and females when normalized to LBM (98). Thus, although ET females may not be able to increase LV wall thickness and mass to the same extent as ET males, they may posses similar capacities to increase LV dimensions, when expressed relative to LBM.

iii) Gender differences in the augmentation of SV during exercise

Using radionuclide angiography, investigators have shown that although males and females achieve similar relative increases in $S \mathrm{~V}, \mathrm{Q}$ and a- $\mathrm{vDO} \mathrm{O}_{2}$ during progressive upright or supine $(1,81,118125,318)$ exercise to maximum, they rely on different mechanisms for 
augmenting these responses. For instance, Higginbotham et al. (125) examined healthy sedentary males and females $\left(32-68 \mathrm{yr}\right.$, mean $52 \pm 11 \mathrm{yr}$ ) who were matched for peak $\mathrm{VO}_{2}$ during progressive upright cycling exercise. The males augmented their SV with a progressive increase in EF from rest to peak exercise, with little change in end-diastolic counts. Whereas, the females who achieved a similar relative increase in SV, relied on a $30 \%$ increase in end-diastolic counts from rest to peak exercise without a change in EF. Hanley et al. (118) found similar results when they examined healthy males and females $(<50 \mathrm{yr}, \overline{\mathrm{x}}$ $=40 \mathrm{yr}$ ) during supine exercise. They observed a lower resting EF in the males and a greater increase in EF from rest to peak exercise than the females. Whereas, the females displayed a smaller resting LVEDV and a larger increase in LVEDV from rest to peak exercise than the males. These two investigations suggest that females rely to a greater extent on the FrankStarling mechanism to augment SV, while males rely more heavily on increasing EF.

Similarly, Adams et al (1) examined healthy males (30 $\pm 10 \mathrm{yr})$ and females (33 \pm 14 yr) during supine exercise. Both the peak exercise EF and the increase in EF from rest to peak exercise was larger in the males. However, since the females maintained their EDV, while the males showed a decrease from rest to peak exercise, the percent changes in $S V$ and $Q$ from rest to peak exercise were not gender-related. Regression analysis showed that gender. but not age was a significant predictor of the change in EF with exercise. Likewise, Fleg et al. (81) examined healthy males and females (22-68 yr) during upright cycle ergometer exercise and showed that although there was no gender difference in peak exercise EF, the males had a lower resting EF and a greater increase in both $E F$ and $Q$, from rest to peak 
exercise. Younis et al. (318) also observed that during multi-stage upright cycle ergometer exercise, both peak exercise EF and the change in EF during exercise was larger in males. However, in contrast to the previous investigations, they observed similar increases in EDVI during exercise in both genders ( $54 \pm 4 \mathrm{yr}$ ). Lastly, Sullivan et al. (268) reported that males and females (20-70 yr, $\overline{\mathrm{x}}=38 \mathrm{yr})$ showed similar increases in both EF and EDVI during upright progressive maximal cycle ergometer exercise. Investigations in which younger ET and sedentary females ( $20 \mathrm{yr})$ were examined showed that despite larger LV masses and LVEDD in ET females, no differences were observed in systolic LV function expressed as \%EF or as \%fractional shortening $(72,200,229,231,319)$.

In overview, the majority of investigators have shown that independent of body position or age, females rely to a greater extent on the Frank-Starling mechanism to augment their SVmax, while males rely on changes in $\mathrm{EF}(1,118,125,81)$. However, the overall picture from these investigations is controversial. For instance, since there is a positive relationship between the changes in EF and peak workloads, and since numerous investigators have examined females at lower absolute submaximal and peak workloads than males $(1,66$, $130,258,318$ ), this could explain some of the gender differences in EF. Additionally, it is interesting to note that in the two investigations using supine exercise, the males showed a decrease in LVEDV from rest to peak exercise, which is contradictory to other investigations of males $(123,212)$, while the females showed an increase in LVEDV (by $\sim 5-10 \%$ ). Further, since LV compliance, BV and heart size affect EDV, and since contractility and afterload influence $\mathrm{EF}$, any of these variables may be responsible for the observed gender differences. 
For instance, since in females, but not males, EDV increased in excess to the already enhanced ventricular filling associated with supine exercise $(1,118)$, females may benefit from greater ventricular compliance that males in response to dynamic exercise (although no investigators have examined this to date). Additionally, since females have a smaller BV, and heart mass than males, it is possible that the smaller EF may be a normal response to different loading conditions in females compared to males.

Although maximal systolic blood pressure (SBPmax) is lower in younger females compared to age-matched males (mainly due to a females' smaller Qmax) $(51,130,181)$ this gender difference decreases around the age of menopause $(50 \mathrm{yr})(51,107,190,191)$. A larger age-related increase in SBP has been observed in females, which was mediated by a larger age-related increase in systemic vascular resistance compared to males $(84,102,181)$. For instance, an investigation on peripheral vascular function in younger and older males and females, indicated greater age-related reductions in maximal calf vasodilatory capacity, which was associated with greater age-related increases in BP, in ET and NA females compared to similarly trained males (181). However, this effect was not observed in those females treated with ERT (181). In agreement, others have shown lower resting and exercise BP responses in postmenopausal females $(48-50 \mathrm{y \tau})$ after $\operatorname{ERT}(175,303)$. Evidence from animal research suggests that estrogen may influence endothelium and non-endothelium dependent vasodilation in coronary arteries, such that estrogen may modulate vascular tone (135). Schunkert et al. (244) observed that ERT decreased vascular reactivity through its blunting effect on renin secretion, which resulted in decreased catecholamine secretion (epinephrine 
and norepinephrine) and decreased $H R$ in postmenopausal females.

In summary, the mechanism responsible for the gender difference in $Q \max$ is a difference in SVmax. The mechanisms responsible for the gender difference in SVmax are differences in body mass and composition, which account for $\sim 50 \%$, differences in heart size, which accounts for $\sim 10 \%$, and differences in BV (refer to following section). In addition, differences in LV compliance, contractility and afterload may contribute to gender differences in the regulation of SV throughout exercise to max.

\section{Age-Related Changes in Qmax}

Cardiovascular reserve declines as a function of age in healthy normotensive sedentary individuals who have been rigorously screened for the presence of CAD. It has been well documented that the age-related decline in $\mathrm{VO}_{2} \max$ is accompanied by a decline in HRmax $(81,115,117,122,205,222,318)$. However, controversy exists regarding the age-related changes in Qduring exercise. This topic is confounded by differences in the criteria employed for the selection of the participants (ie. screening for disease, fitness level and body composition), body position during the study, exercise intensity (relative versus absolute), and the method used to measure $Q$. While some investigators have observed age-related reductions in exercise $Q(81,110,117,122,124,139,205,222,267,318)$, others have observed no changes in exercise $Q$ with aging $(8,100,224)$.

i) Investigations showing an age-related decline in 0 max

Investigators showing a reduction in Qmax with advancing age observed reductions in $H R \max (81,117,122,205,222,226,318)$ and $S V \max (205,222)$. Some of the earlier 
investigations proceeded the availability of screening techniques (ie. thallium scintigraphy), and therefore, may have inadvertently included individuals with $\operatorname{CAD}(8,110,115,139,267)$, and thus, will not be reviewed here.

Rivera et al. (222) examined masters male athletes $(66 \pm 8 \mathrm{yr})$ and younger male athletes ( $32 \pm 5 \mathrm{yr}$ ) during treadmill exercise, with the acetylene rebreathe manoeuvre. In masters males compared to younger ET males they observed a $36 \%$ lower $\mathrm{VO}_{2} \max$, which was due to a $28 \%$ lower $\left(\max \left(18.2\right.\right.$ vs $\left.25.31 \cdot \mathrm{min}^{-1}\right)$ and an $11 \%$ lower $\mathrm{a}-\mathrm{v} \mathrm{DO}_{2} \max (16.6$ vs $\left.18.7 \mathrm{ml} \cdot 100 \mathrm{ml}^{-1}\right)$. The lower Qmax resulted from a $15 \%$ lower $\mathrm{SVmax}(117 \mathrm{ml}$ vs $137 \mathrm{ml})$ and a $16 \%$ lower $\mathrm{HRmax}$ (154 vs $185 \mathrm{bpm}$ ). They concluded that $\mathrm{VO}_{2} \max$ is lower in older compared to younger ET males due to a decreased capacity to both deliver and extract oxygen. In support of the later results, Ogawa et al. (205) examined healthy sedentary males (23-68 yr) and females (20-72 yr) and ET males (21-72 yr) and females (18-63 yr) during progressive treadmill exercise to maximum, using the acetylene rebreathe method. In older ET males compared to younger ET males, they observed a $28 \%$ lower $\mathrm{VO}_{2} \max$, which was due to a $25 \%$ lower $Q \max \left(20.5\right.$ vs $\left.27.41 \cdot \mathrm{min}^{-1}\right)$ and a $5 \%$ lower a-v̄ $\mathrm{vO}_{2} \max (14.7$ vs 15.5 $\left.\mathrm{ml} \cdot 100 \mathrm{ml}^{-1}\right)$. The lower $\mathrm{Qmax}$ was due to a $19 \%$ lower $\mathrm{SV} \max (124 \mathrm{ml}$ vs $154 \mathrm{ml})$ and a $7 \%$ lower HRmax (165 vs $178 \mathrm{bpm}$ ). Thus, similar to the Rivera et al. (222) study, they concluded that $\mathrm{VO}_{2}$ max is lower in older compared to younger ET males due to a decreased capacity to both deliver and extract oxygen.

In contrast, Heath et al. (122) examined masters males (59 $\pm 6 \mathrm{yr})$ and younger ET males (who were matched to the training programs of the masters athletes) (18-27 yr) and 
sedentary older males (42-62 yr) during treadmill exercise. The two ET groups had similar values for maximal oxygen pulse, suggesting that $\mathrm{SVmax}$ and $\mathrm{a}-\overline{\mathrm{vDO}} \mathrm{O}_{2} \max$ were maintained with aging in the masters males. Thus, the smaller $\mathrm{VO}_{2} \max (\sim 14 \%)$ in the masters males was due to an age-related decline in HRmax (169 vs $197 \mathrm{bpm}$ ). In agreement, Hagberg et al. (117) examined masters males ( $56 \pm 5 \mathrm{yr}$ ), competitive younger runners ( $26 \pm 3 \mathrm{yr}$ ), younger runners who were matched in training and performance to the masters males ( $25 \pm 3 \mathrm{yr}$ ), and healthy older sedentary males ( $58 \pm 5 \mathrm{yr}$ ) during progressive treadmill exercise with the $\mathrm{CO}_{2}$ rebreathing method. Although the three groups of ET males had similar levels of SVmax and $\mathrm{a}-\mathrm{v} \mathrm{DO}_{2} \max$, a smaller $\mathrm{Qmax}(\sim 13 \%)$ due to a lower HRmax $(\sim 12 \%)$ in the masters males compared to the two younger ET groups, accounted for the lower $\mathrm{VO}_{2}$ max $(\sim 16 \%)$ in the masters males. Similar to Heath et al. (122) it was concluded that the masters males did not exhibit the decline in $\mathrm{SV} \max$ and $\mathrm{a}-\mathrm{VDO}_{2} \max$ that occurred with aging in the sedentary males. However, due to the limitation of the measurement technique, these authors were unable to measure $S V \max$ at $\mathrm{VO}_{2} \max$, and thus choose to estimate the SV measurements at 50,60 and $70 \%$ of $\mathrm{VO}_{2} \max$ to represent SVmax. However, this may not be accurate, since as previously discussed, SV may not plateau at submaximal exercise intensities in ET individuals, but may continue to increase up to $\mathrm{VO}_{2} \max (46,105,152,220,222,230,289)$.

In a recent investigation, Fleg et al. (8I) examined 200 healthy sedentary males and females (22-68 yr) at rest and during upright cycle ergometer exercise, using gated radionuclide ventriculography. Although stroke volume index (SVI) was maintained with age, peak cardiac index (CI) decreased, due to a decrease in HRmax. Similarly, Younis et al. 
(318) examined healthy younger males and females $(22 \pm 2 \mathrm{yr})$ and older males and females (54 \pm 4 yr) during upright cycle ergometer exercise using radionuclide ventriculography. Although they observed an age-related increase in peak SVI (although not analyzed statistically), peak CI decreased due to a decrease in peak HR.

Thus, in contrast to the investigations of Ogawa et al. (205) and Rivera et al. (222), results from the later four investigations $(81,117,122,318)$ suggest that the age-related decline in peak $Q$ is due soley to a decline in $H R \max$, while $S V \max$ does not change with age. ii) Investigations showing an age-related maintenance of $O \max$

Investigators showing that $Q \max$ does not decrease with age, show a compensatory increase in SVmax $(8,100,224)$. Rodeheffer et al. (224) and Gerstenblith et al. (100) vigorously screened NA males (25-79 yr) for $\mathrm{CAD}$, according to their histories and results of physical, resting and stress electrocardiogram (ECG), and stress thallium scintigraphy. Using radionuclide ventriculography during upright cycle exercise, they observed a maintenance of $Q$ at rest and during both submaximal and maximal cycle exercise due to an augmented SVmax (which compensated for the decreased HRmax) (183 to $137 \mathrm{bpm}$ ) in the older males. In the younger males, at high workloads, HR increased, while both LVEDV and LVESV decreased, resulting in a plateau in SV. In contrast, the older males whose HR increased less displayed progressive increases in LVEDV, and thus SV, without a plateau throughout exercise to peak effor. However, these investigations have been criticized, since the vigorous screening process may have produced an unusual sample. The concept of restricting analyses of an older population, such that no one shows even minor ECG or 
scintigraphy changes is somewhat artificial, especially given the fact that $25 \%$ of older athletes show some abnormalities in these measurements. In addition, the active sample of older males ( $-65 \mathrm{yr}$ ) had a Qmax of $18 \mathrm{l} \cdot \mathrm{min}^{-1}$ and a SVmax of $140 \mathrm{ml}$. This Qmax is similar to the Q $\max$ of $20.51 \cdot \mathrm{min}^{-1}$ reported for the masters males $(\sim 63 \mathrm{yr})$ in the Ogawa et al. (205) investigation and the $Q \max$ of $18.21 \cdot \mathrm{min}^{-1}$ reported for the masters males $(-66 \mathrm{yr})$ in the Rivera et al (222) investigation. Since a larger Qmax has been consistently observed in ET males compared to NA age-matched males, these results seem strange. Further, the SVmax of the Rodeheffer et al. (224) males is even greater than the SVmax of $124 \mathrm{ml}$ and $117 \mathrm{ml}$ reported for the masters males in the Ogawa et al (1992) and Rivera et al. (222) studies, respectively. Thus, the results of the Rodeheffer et al. (224) and Gerstenblith et al. (100) investigations should be interpreted with caution.

In summary, these investigations suggest that $Q \max$ is maintained with age in rigorously screened healthy older males, due an augmented SVmax which compensates for the decreased HRmax $(100,224)$. These authors postulated that the mechanism for the decrease in cardiac function with age observed by other investigators is subclinical disease.

\section{iii) Mechanisms responsible for the age-related decline in HRmax}

As previously reviewed, HRmax declines as a function of age in both NA and ET males and females $(81,102,117,122,205,222,224,318)$. While an individual of $25 \mathrm{yr}$ has a HRmax of $\sim 195 \mathrm{bpm}$, this is reduced to $\sim 155 \mathrm{bpm}$ by $65 \mathrm{yr}$, regardless of gender or training status (HRmax $=220-$ age $\pm 12 \mathrm{bpm})(5,234)$. Mechanisms responsible for the decreased HR are: 1) an attenuation of the responses of cardiac tissue to $\beta$-AR stimulation $(39,245)$, 
and 2) a decrease in the intrinsic HR (158).

The responsiveness of the cardiac tissue to $\beta$-AR stimulation (catecholamines) is diminished with aging (160). For instance, in aging human lymphocytes (a common model for examining the effect of aging on AR function), a decrease in $\beta$-AR affinity for agonists has been observed without significant changes in the $\beta$-AR density, thus suggesting a decline in post-synaptic responsiveness to $\beta$-AR stimulation $(84,158)$. Experiments show that the HR response to a bolus infusion of isoproterenol ( $\beta-A R$ agonist) in humans decreases with aging $(157,158,266)$. This in turn results in decreased contractile force and contraction-relaxation speed during exercise and thus, a decreased HRmax (156).

Additionally, an age-related decline in the intrinsic sinus node rate results from morphological and electrophysiological changes in the sino-atrial (SA) node and other portions of the conduction system (234). Histological analyses of hearts show substantial reductions in the total number of pacemaker cells in the SA node, beginning by $60 \mathrm{yr}$, and by $75 \mathrm{yr},<10 \%$ of the cells normally observed in a younger individual remain (156). In addition, with advancing age there is an increase in elastic and collagenous tissue in all parts of the conduction system and fat accumulates around the SA node, with the pacemaker cells showing increases in connective tissue infiltration. However, the AV node maintains its cell population in elderly subjects, although age-related increases in AV node transmission is common. The bundle of His remains morphologically intact, although there is often a loss of Purkinje fibres, particularly in the left bundle, which sometimes can be reduced by up to $50 \%$ $(84,156)$. 


\section{Age-Related Changes in SVmax}

\section{i) Mechanisms responsible for an age-related decline in SVmax}

As previously reviewed, some investigators have observed age-related decreases in SVmax in NA and ET males and females $(205,222)$. According to the Frank-Starling mechanism, decreases in SVmax may result from decreases in diastolic filling (and thus, preload), increases in BP (and thus, afterload) and/or decreases in myocardial contractility. Thus, age-related changes in both systolic and diastolic function could explain the difficulties in maintaining SVmax with aging. Age-related decreases in: $\beta$-AR responsiveness, arterial distensibility and ventricular compliance (201) contribute to increases in BP, TPR and thus, afterload which in turn, results in ventricular hypertrophy and decreased myocardial contractility (156).

During exercise, ESV fails to decrease to the same extent in older compared to younger individuals $(179,224,241)$, resulting in smaller increases in $E F(E F=(E D V-$ ESV)/EDV) from rest to peak exercise $(125,215,224,318)$. For instance, peak EF values ranging from 55-72\% with progressive declines after $60 \mathrm{yr}$ have been reported $(179,215)$. Diminished $\beta$-AR responsiveness $(156,215)$ and/or increased afterload $(316)$ have been implicated as possible factors responsible for this change.

As previously discussed, $\beta$-AR inotropic and chronotropic responsiveness of cardiac myocytes, atrial pacemaker cells and vascular smooth muscle cells are reduced progressively with advancing age $(157,160)$. For instance, after an infusion of isoproteronol ( $\beta$-AR agonist), ESV failed to decrease in older males to the same extent as it did in younger males, 
possibly due to age-related decreases in $\beta$-AR responsiveness of the LV (266). Additionally, during upright cycle exercise an acute $\beta$-AR blockade with propranolol in younger individuals attenuated the increases in HR, EF and SBP/ESV (measure of LV contractility) and the reduction in ESV, which would have otherwise have been observed (219). Thus, the hemodynamic profile of older individuals during exercise, is similar to that of younger individuals on $\beta$-AR blockade. Hence, it is possible that the mechanism responsible for the lower SVmax observed in older individuals is a diminished ability to compensate for lower filling rates (refer to the Diastolic Filling Rate section) by sympathetic means.

Also, afterload, as measured by total peripheral (or systemic) vascular resistance has been shown to increase with age at the same relative exercise intensity $\left(\% \mathrm{VO}_{2} \max \right)$ and during maximal exercise $(51,190,205)$. Mechanisms responsible for the increase in TPR are increases in arterial stiffness $(102,117,284)$ and/or decreases in peripheral vasodilatory capacity (primarily in the active skeletal muscles) $(102,117,205,222,224)$. Age-related biochemical alterations in the arterial walls, such as wall thickening, loss of elastin filaments and partial replacement with collagen contribute to the increased arterial stiffness $(84,102)$. Additionally, although there is a reduction in the venous response to $\beta$-AR stimulation (dilation/ relaxation), the $\alpha$-adrenergic (constrictor) response appears to be maintained (156).

Aortic impedance is also increased during exercise due to structural changes, such as dilation, elongation and increased wall stiffening, in addition to decreased $\beta$-AR chronotropic responsiveness (316). This impedance to ejection, results in a reduced shortening and a relative increase in ESV, such that, systolic emptying, and thus SVmax will be limited. 
ii) Mechanisms responsible for an age-related maintenance of SVmax

As previously reviewed, some investigators have observed an age-related maintenance or increase in SVmax in both NA and ET males and females $(81,100,117,122,224,318)$. Most researchers agree that in contrast to younger individuals, there is little or no reduction in ESV with higher levels of exercise intensity in older individuals, and increases in SV are accomplished by LV cavity dilation, an elevated EDV and use of the Frank-Starling mechanism $(123,179,224)$. It has been suggested that during exercise, the mechanism through which older subjects achieve a high $Q$ shifts from a dependence on catecholaminemediated increases in HR and inotropy to a greater reliance on the Frank-Starling mechanism $(100,243)$.

For instance, in younger individuals, the same $Q$ is achieved during upright cycle exercise in the presence of acute $\beta$-AR blockade with propranolol as in the absence of $\beta$-AR blockade. However, in the presence of $\beta$-AR blockade, the increase in HR, EF and SBP/ESVI and the reduction in ESV which would have otherwise been observed (without $\beta$-blockade), are markedly reduced, while EDV is increased, thus permitting an increase in SV (219). Therefore, cardiac dilation and the use of the Frank-Starling mechanism, augment SV and compensate for the reduced HR (due to a deficit in $\beta$-AR stimulation) and in turn, maintain $Q$.

Rodeheffer et al. (224), observed in older sedentary males compared to younger sedentary males, an increase in resting EDVI (5-10\%), no difference in submaximal EDVI and an 20-30\% greater increase in EDVI at exhaustion. It was postulated that the augmentation 
in EDVI during exercise may be an adaptation through which the age-related decline in HRmax is offset in order to maintain or even increase SVmax via the Frank-Starling mechanism. Similarly, in the investigation by Fleg et al. (81) and Younis et al. (318), the agerelated maintenance or increase in SVI, respectively, in older sedentary males, was shown to be due to an age-related increase in peak EDVI despite an increase in peak ESVI and a decrease in peak EF. Thus, the relationship between SVI and EDVI was shifted upward and rightward in the older males at fixed workloads such that they utilized the Frank-Starling mechanism to a larger extent than the younger males to augment their exercise SVI.

In contrast to these findings in males, Fleg et al. (81) observed no age-related increases in EDVI at peak exercise in older females. However, they did observe a change in EDVI expressed from rest to peak exercise in the older females, and reasoned this was due to similar increases in LV dilation in males and females from rest to peak exercise. To observe whether supplemental estrogen alters the hemodynamic responses to exercise, they compared the pre-menopausal females on OCA with age-matched non-users and observed no hemodynamic differences at rest or during exercise between the two groups. Similarly, they compared the postmenopausal females on HRT with those who were not $(>60 \mathrm{yr})$, and observed that the only significant difference was in the change in ESVI from rest to peak effort, which decreased by $6 \mathrm{ml} / \mathrm{m}^{2}$ in the non-supplemented group and by only $1 \mathrm{~mL} / \mathrm{m}^{2}$ in the HRT using group.

Thus, it appears that in males, because of an age-related decrease in myocardial contractile reserve and/or an increase in afterload, the aging heart dilates and contracts from 
a greater prebad than does the heart of a younger male. In support of this, at rest $(84,155$, 224) and during supine or semi-supine exercise (110, 179, 241), LVEDV and LVEDD were larger in older compared to younger healthy males. It has been postulated that the age-related increase in LV eccentric hypertrophy/ dilation may be the result of chronic stretch, thus allowing SV to be preserved at end-diastole (224). Also, in contrast to the decreased contractile response of the LV to $\beta-A R$ stimuli, the relaxation response of the $L V$ to $\beta-A R$ stimuli is maintained (lusitropic responsiveness) (158), and thus, may contribute to a maintenance of an efficient use of preload reserve during exercise.

In the Seals et al. (245) study, although both the older sedentary males and masters males tended to rely on the Frank-Starling mechanism to augment SV, a larger rise in SV for a given increase in EDV, was observed in the masters males. In addition, a greater exercise induced augmentation in EF, and a larger decrease in ESV and diameter, was observed in the masters males, compared to the sedentary males. These authors postulated that the augmented ESV response observed in the masters males was due to enhanced myocardial contractility. Lakatta (160) suggested that improvements in systolic function could contribute to the training induced increases in SVmax in older ET individuals by counteracting the age related decrease in sensitivity to catecholamines.

In agreement, Fleg et al. (82) observed that the larger SVI in the masters males compared to the sedentary age-matched males, was the result of an $11 \%$ larger EDV and a 7\% higher EF. They found greater decreases in systemic vascular resistance from rest to peak exercise, as well as at peak exercise in the older ET males compared to the NA age-matched 
males, suggesting that a decreased afterload may play a role in augmenting the SV of older ET males. In agreement, older ET males (54-75 yr) displayed decreased arterial stiffness, despite similar BP compared to NA age-matched males (284). Similarly, Martin et al. (181) observed that calf vasodilatory capacity increased during submaximal exercise in ET individuals regardless of gender or age. Other investigators who have examined older athletes have shown significantly lower BP and systemic vascular responses to exercise compared to age-matched controls $(117,122)$. The results of these investigations suggest that the aging process may not be directly responsible for the increase in MAP. However, it was postulated that arterial stiffness and exercise capacity may be in part genetically predetermined, such that greater compliant vessels of ET older individuals may be a factor that allows them to exercise successfully $(117,122)$.

Thus, it appears that in comparison to younger sedentary males, older sedentary males rely to a greater extent on the Frank-Starling mechanism to augment their SVmax, due to age-related decreases in LV responsiveness to $\beta$-AR stimulation and/or increases in afterload. In addition, endurance training in older males may lead to an even greater capacity to use the Frank-Starling mechanism, in addition to enhancing LV systolic function, which may result from training-induced LV volume overload hypertrophy, enhancements in myocardial contractility, and/or decreases in afterload. However, further research is needed in this area before any definitive conclusions may be drawn.

In summary, the results of these investigations suggest that regardless of gender or training status, Qmax decreases with age due to a decreased $\operatorname{HRmax}(81,117,122,222,205$, 
$318)$ and $S V \max (81,205,222)$. In addition, declines in $\mathrm{a}-\mathrm{vDO} \mathrm{v}_{2} \max$ may also be a factor $(205,222)$. However, in comparison to NA individuals who show age-related decreases in myocardial contractility, and increases in afterload, these responses may be attenuated with endurance training, thus resulting in augmented LVEDV and LVESV responses during exercise, and ultimately a greater $\mathrm{SVmax}$ and thus, $\mathrm{VO}_{2} \max$.

\section{Cardiac Time Intervals in ET Individuals and the Relationship to Gender and Age}

Although cardiac time intervals (CTI) were originally studied invasively (173), they are now more commonly measured non-invasively, allowing for the examination of cardiac function not only at rest, but also during incremental exercise to maximum (292). Thus, CTI have been used to examine the effects of endurance training on cardiac function (105). The CTI as originally defined, are determined from simultaneous high speed recordings of an ECG, phonocardiogram, and carotid arterial pulse transducer (13), and correlate highly with internal measurements of cardiac function $(168,185,292)$. A carotid pulse transducer is one method used for measuring LVET. However, an ear pulse densitograph is a more suitable method for measuring LVET during exercise, since body movement does not effect the reliability of the tracings and more comfort is provided for the participant $(76,161)$.

The cardiac cycle consists of a period of relaxation (diastole) followed by a period of contraction (systole). During exercise, cardiac function is altered such that the periods of diastole and systole change in response to the increased cardiovascular demands imposed upon the system. The time periods of interest measured from CTI are: LVET, PEP and DT. The LVET and PEP are inversely and linearly related to HR (293). Strong negative 
correlations exist between HR and LVET $(r=-0.92)(195)$ and between HR and PEP $(r=-$ 0.77) (292). In contrast, a non-linear relationship exists between DT and HR, such that small variations in HR may lead to significant changes in DT. The length of a long cardiac cycle (ie. resting) is determined more by the duration of DT, than by the durations of either LVET or PEP (173). Furthermore, as the cycles shorten with increasing HR, DT shortens (ie. at HR $>100 \mathrm{bpm}$, DT occupies $<1 / 2$ of the cardiac cycle) much faster than either LVET or PEP, with the latter two variables occupying an ever increasing proportion of the cardiac cycle. Thus, to interpret changes and/or differences in CTI between individuals at rest, correction must be made for variations in HR (292).

\section{Cardiac Time Intervals in ET Compared to NA Individuals}

\section{i) LVET}

LVET varies directly with enhanced ventricular filling, $S V$ and afterload and inversely with inotropy $(177,292,293)$. For instance, during exercise, an increased venous return, preload and thus SV (Frank-Starling mechanism), or an increased BP, and thus afterload, would prolong LVET, while positive inotropic effects induced by AR stimulation, would shorten LVET (177). Numerous investigators have shown a direct relationship between LVET and preload (BV) $(76,169,195)$. Further, strong positive correlations have been observed between BV and LVET (ie. $r=0.94$ and $r=0.86$ ) (137). The majority of investigators have shown that ET individuals have a prolonged LVET at rest and during exercise compared to NA individuals $(76,105,152,154,195,311)$. The lengthened LVET is likely the result of an elevated SV and thus BV of ET individuals (177). It has been postulated that 
a longer time is required for emptying a larger volume of blood per beat. In contrast, some investigators observed no differences in LVET or DT in ET compared to NA individuals $(153,279,309,320)$.

\section{ii) $\quad$ PEP}

The duration of PEP is affected by the magnitude of ventricular filling (195) and myocardial contractility $(44,177,320)$. For instance, enhanced ventricular filling, which is determined by BV, will activate the Frank-Starling mechanism and increase myocardial contractility, thereby shortening PEP (195). Since chronic endurance training results in a larger BV, PEP would be expected to decrease. In general, the shortening of both PEP and LVET are used to evaluate positive inotropic effects, while a prolongation of PEP or an increase in PEP/LVET are used to evaluate negative inotropic effects $(169,292)$. Additionally, acute increases in afterload (ie. BP) prolongs PEP, has no effect or slightly prolongs LVET, and increases PEP/LVET.

There seems to be a great deal of controversy regarding the effect endurance training has on PEP. At matched HR during exercise Cox et al. (44) observed no differences in PEP in male cyclists compared to NA males. Cox et al (44) postulated that since PEP is primarily affected by inotropic stimulation, and since the ET and NA males were matched for HR at all levels of exercise, no difference in adrenergic status would be expected to occur between groups, and thus similar PEP values should have resulted. In addition, circulating levels of catecholamines are directly related to the relative intensity of the exercise. If the relative $\mathrm{VO}_{2}$ values are similar between groups at each $H R$, then the catecholamine responses should also 
be similar. In agreement, Lamont et al (157) observed no change in resting PEP after 13 wk of intense swim training in females.

In contrast, Zoneraich et al. (320) observed a prolonged PEP in male marathon runners compared to NA males. Krip et al. (152) also observed a prolonged PEP in ET compared to NA males. Since PEP is primarily affected by inotropic stimulation (291), a prolonged PEP may resuit from a decrease in sympathetic activation. Wolfe (311) used CTI to assess the cardiac function in males and females and observed a lower PEP/LVET in those with a higher aerobic capacity, compared to those with a lower aerobic capacity, suggesting a decreased afterload or positive inotropic effect.

iii) $\mathrm{DT}$

DT is primarily affected by changes in LVET and PEP. For instance, a shortened LVET and PEP would prolong DT, while a prolonged LVET and PEP would shorten DT. It was postulated that a shortened DT in ET may result from an augmented preload induced dilation of the LV (278), or a greater rate of LV relaxation due to greater elastic recoil and enhanced LV pressure $(25,186,315)$

As a result of a lengthened LVET in ET individuals, the period of systole constitutes a larger percentage of the cardiac cycle, thus decreasing the time available for diastole. Investigators have observed that DT is shorter in duration during exercise in ET compared to NA individuals $(76,105,152)$. Although a decreased DT in ET individuals seems to be counterproductive for increasing EDV and thus SV during exercise, investigators have observed an enhanced DFR in ET compared to NA males $(105,152)$ which would thus, 
compensate for the shorter DT. Levy et al. (167) observed that in response to 6 mo of endurance training, younger $(\sim 28 \mathrm{yr})$ and older males $(-68 \mathrm{yr})$ displayed no change in exercise DT. This may suggest that more than 6 mo of endurance training is required before a change in exercise DT is evident. While some investigators have observed a prolonged DT at rest in ET compared NA individuals $(75,76,152,167)$ others have seen no difference in resting DT (79). The authors postulated that differences in DT were due to the frequency of training (79). Some of the variablility in results may also be partially due to a failure to control for the effects of resting HR on DT, since unlike the other resting CTI (LVET, PEP) no correction factor has been developed for DT. For instance, Levy et al. (167) postulated that the prolonged resting DT observed in response to 6 mo of endurance training, in younger ( 28 yr) and older males ( $-68 \mathrm{yr})$ was due the bradicardic (HR lowering) effect of endurance training, which resulted in a lengthening of the entire cardiac cycle and thus, DT. Additionally, differences in the experimental design, the fitness level of the participants and the type of exercise performed by the participants may be responsible for the diversity of results.

\section{Gender Differences in CTI}

Although CTI have been measured in ET males, to date there have been no investigations of CTI in females during incremental exercise to maximum. Independent of HR, LVET is significantly longer in females compared to males $(171,294,301)$. For instance, Falsetti et al. (74) examined male and female competitive cyclists (19-31 yr), with $\mathrm{VO}_{2} \max$ values of 61 and $42 \mathrm{ml} \cdot \mathrm{kg}^{-1} \cdot \mathrm{min}^{-1}$, in the males and females, respectively. After 
correction was made for resting HR, they observed PEP values of 122 and $118 \mathrm{msec}$ and LVET values of 396 and $410 \mathrm{msec}$, in the males and females respectively. Although statistical analysis was not conducted, it appears that LVET is longer in the females. In agreement, Willems et al. (301) examined elderly males $(72 \pm 5 \mathrm{yr})$ and females $(70 \pm 4 \mathrm{yr})$, and found that at all HR, LVET was statistically longer in the females by $12.9 \mathrm{msec}$ (which was $4 \%$ of the observed LVET at a HR of $74 \mathrm{bpm}$ ). In agreement, Lombard et al. (171) observed that LVET was longer in females compared to males at matched cycle lengths, independent of posture (ie. recumbent, sitting, standing). Lombard et al. (171) suggested that this gender difference may be due to the smaller heart size in proportion to the body size of a female, compared to a male. They hypothesized that if similar amounts of blood were sent to the heart, a female's smaller heart would be enlarged more than a male's heart by the entering blood, and thus, would need more time to eject the blood (171). Lombard et al. (171) also discussed the phenomena which they termed "crossing of the curves" where at very low HR the LVET of a female not only approaches that of a male, but becomes shorter than a male. They did not offer any explanation for this. Although DT has not previously been measured to date in females, a prolonged LVET in females compared to males would be expected to result in a subsequent shortening of DT.

An endurance training program in physically active younger female swimmers (18-32 yr), resulted in enhanced resting LV function as demonstrated by a lengthened LVET, with no change in PEP/LVET (154), suggesting a prolonged PEP. The lengthened LVET was attributed to an increased SV, which was reflected by an increased echocardiographic 
LVEDD in the female swimmers. This is in agreement with Cox et al. (44) who observed a lengthened LVET in ET compared to NA males, and with Krip et al. (152) who observed a prolonged PEP in ET compared to NA males. Thus, it appears that ET females display similar adaptations in their CTI as ET males, and as such, they should also display the characteristic shortening of DT.

\section{Age-Related Changes in CTI}

To date there have been no investigations of CTI during incremental exercise to maximum which compare ET females across age groups. As a consequence of aging, NA older individuals display a prolonged LVET and PEP at the expense of DT at rest, compared to NA younger individuals, with this difference becoming enhanced during exercise $(251,286$, 301). For instance, Willems et al. (301) examined a combined group of younger (302) and elderly individuals (301) and observed a small but significant influence of age on LVET, independent of HR and BP. At all HR, LVET was $10-20 \mathrm{msec}$ longer than normal values calculated by equations of Weissler et al. (293), and was prolonged by $2 \mathrm{msec} \cdot \mathrm{decade}^{-1}$. In an examination of children, adolescents and adults, Wanderman et al. (288), observed a distinct tendency towards lengthening of LVET and PEP with advancing age, independent of HR. Shaw et al. (251) examined males (20-89 yr) and observed a prolonged PEP of 4 msec $\cdot$ decade ${ }^{-1}$ from ages 30-60 yr, and a shortening, thereafter. Similarly, Montoye et al.

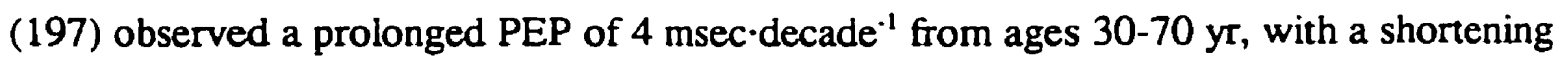
thereafter. Vavra et al. (286) observed a slight lengthening of LVET $(20-30 \mathrm{msec})$ and a slight shortening of PEP, especially with exercise in older males (45-55 yr) compared to boys 
(11-14 yr) and younger males and females (20-30 yr). These authors also observed a shortening of DT, such that the boys' DT represented $35 \%$ of the cardiac cycle, while the older males' DT represented only $25 \%$ of the cardiac cycle.

Possible reasons for the age-related changes in LVET may be a decline in myocardial contractility, due to decreased $\beta$-AR responsiveness, or an increased afterload to the left ventricle, resulting from increased aortic impedance or increased peripheral resistance. Evidence for the latter comes from Willems et al. (301) who observed a small but significant correlation between SBP and LVET after correction for HR and age by multiple regression analysis, such that for each $10 \mathrm{mmHg}$ increase in SBP, LVET was prolonged by $1.34 \mathrm{msec}$. Similarly, Weissler (292) and Shaw et al. (251) observed a prolonged LVET in participants with either a SBP > $140 \mathrm{mmHg}$ or an increased MAP, and a prolonged PEP in those with either a DBP > $90 \mathrm{mmHg}$ or an increased MAP. An age-related lengthening of PEP may also be the result of a prolonged duration of contraction observed in older compared to younger individuals (refer to Diastolic Filling Rate section).

A prolonged PEP and LVET, correspondingly reduce DT. During exercise, a shorter DT in older individuals may tend to impede LV filling at high HR (when additional increases in afterload occur) and reduce $S V$ in a situation in which an increased $Q$ is required (156). Since CTI have not previously been measured in older ET individuals, it is unknown whether they exhibit similar age-reiated changes. 


\section{Blood Volume in ET Individuals and the Relationship to Gender and Age}

\section{Blood Volume in ET Compared to NA Individuals}

It has been well documented that one adaptation to endurance training is an increased BV. Both cross-sectional $(15,59,149,265)$ and longitudinal investigations $(23,37,103$, $113,192,206)$ consistently show this effect. High correlations have been reported between $\mathrm{BV}$ and $\mathrm{VO}_{2} \max$ in younger males $(r=0.78)(35)$ and in older females $(r=0.79)(265)$. Blood volumes in the range of 90 to $104 \mathrm{ml} \cdot \mathrm{kg}^{-1}$ have been reported in younger ET males compared to $\sim 75 \mathrm{ml} \cdot \mathrm{kg}^{-1}$ in age-matched NA males $(15,59,149)$, while BV of $89 \mathrm{ml} \cdot \mathrm{kg}^{1}$ have been reported in younger and older ET females, compared to $62 \mathrm{ml} \cdot \mathrm{kg}^{-1}$ in age-matched NA females $(23,136,149,192,265)$. Thus, cross-sectional investigations reveal that ET males have a BV that is $20-25 \%$ larger than age-matched NA males $(15,59,149)$, while ET females display a BV that is $\sim 30 \%$ larger than age-matched-NA females (149).

\section{Functional Importance of an Enhanced BV}

It has been postulated that an elevated BV will improve endurance performance via enhancements in both cardiovascular and thermoregulatory mechanisms during exercise (35, 37). First, a larger BV elevates venous return, and thus ventricular preload, which is reflected in an increased EDV $(128,140)$. An increased EDV elicits a more forceful contraction (due to the lengthening of the myocardial fibres) and thus, increases $S V$ and $Q(142,128)$. An augmented BV has also been associated with an elevated central venous pressure, which increases the pressure gradient to the right atrium and thus, increases SV (128). Lastly, an augmented BV has been shown to result in decreased systemic vascular resistance and arterial 
pressure, which would decrease afterload and thereby, enhance SV (140). Along with the elevation in SV, HR is reduced at the same relative submaximal intensity (37).

Secondly, an elevated BV contributes to an enhanced capacity for heat dissipation during exercise $(35,119,128,140)$ via increased blood flow to the skin and enhanced sweat rates (more water is available for sweating), thereby decreasing core temperature during exercise (88). It was postulated that these changes improve cardiac reserve and thermoregulation and allow for a greater capacity to perform prolonged intense endurance exercise.

\section{Mechanisms Responsible for the Enhanced BV in ET Compared to NA Individuals}

Endurance trained individuals have a larger BV, primarily due to an expanded PV $(127,149)$ and red cell volume $(\mathrm{RCV})(2,24,192)$. Endurance training stimulates movement of fluid into the vasculature, thereby eliciting an increase in PV. A primary mechanism for the increase in PV in ET individuals is an increase in total circulating plasma proteins, which thereby elevates the oncotic pressure across the capillary membranes and promotes an osmotic shift of water in to the vascular space. For instance, Freund et al. (93) found that, although the decrease in PV during maximal treadmill exercise was similar in ET and NA individuals, the PV of ET individuals was expanded $1 \mathrm{hr}$ post-exercise, whereas NA individuals did not show this effect. Although there was no difference between groups in the plasma levels of vasopressin (ADH), renin, ANF or aldosterone during exercise or recovery, the ET individuals had a larger plasma protein concentration during recovery, which was associated with a larger PV. It was proposed that there may be two phases involved in the 
increase in total circulating plasma proteins. The first phase, which occurs early after the onset of exercise, may involve a shift of protein and fluid from the interstitial spaces to the intravascular spaces via lymphatic flushing (247). The second slower phase may involve de novo synthesis of protein with a resultant expansion of total body water (35).

Additionally, during $24 \mathrm{hr}$ post-exercise, thirst is increased, thus aiding in fluid replacement, and urine output is reduced, thereby increasing renal retention of water and $\mathrm{Na}^{+}$ (35). These two mechanisms help to offset the increased water and $\mathrm{Na}^{+}$losses due to the enhanced rate of sweating observed in ET compared to NA individuals during exercise (35). Plasma levels of $\mathrm{ADH}$, renin, and aldosterone increase during exercise $(37,41)$ and remain elevated for at least $2 \mathrm{hr}$ post-exercise. The renin-angiotensin-aldosterone system leads to $\mathrm{Na}^{+}$retention, which in turn, results in a fluid shift into the vasculature, while $\mathrm{ADH}$ conserves water by favouring the reabscrption of solute free water. It has been postulated that the renal $\mathrm{Na}^{+}$reabsorption, may be mediated by increased receptor sensitivity to aldosterone and/or increased number of available receptors (36).

Luetkemeier et al. (174) postulated that an aldosterone-sodium retention system may be an important factor in the exercise-induced hypervolemia. When NA males were given an aldosterone antagonist (spironolactone) during the first $3 \mathrm{~d}$ of endurance training, greater renal $\mathrm{Na}^{+}$and water excretion were observed and normal PV expansion was attenuated (by $67 \%$ ) compared to a control group. However, by the end of the third day of training, the total amount of circulating plasma proteins of the two groups were similar. It was concluded that two-fifths of the increase in PV resulting from $3 \mathrm{~d}$ of endurance cycling was due to 
aldosterone activity, while the remaining three-fifths was accounted for by the expansion of intravascular protein.

\section{Gender Differences in BV in ET Individuals}

Endurance trained males and females display similar training-induced augmentations in BV, expressed relative to body mass (149). For instance, in a longitudinal investigation, 2 mo of cycle ergometer training, in previously sedentary individuals, resulted in expansions of $\mathrm{BV}, \mathrm{PV}$ and $\mathrm{RCV}$ by 7,8 and $7 \%$ in females compared to 10,11 and $9 \%$ in age-matched males (2). Similarly, in a more recent study, Mier et al. (192), observed that $10 \mathrm{~d}$ of cycle ergometer training in previously sedentary males $(29 \pm 2 \mathrm{yr})$ and females $(25 \pm 2 \mathrm{yr})$ resulted in a significant increase in $\mathrm{BV}$ and $\mathrm{SV}$ in both groups, and although males had a larger absolute BV, when expressed relative to body mass, no gender differences existed. It was postulated that males and females posses similar cardiac reserve capacities for increasing BV as a result of endurance training (192).

i) Influence of hormonal variations during the menstrual cycle and the use of OCA

During the menstrual cycle, increased hormone levels (estrogen and progesterone) are associated with a retention of fluid and electrolytes as well as with an increase in permeability of the capillaries. Therefore, fluctuations in BV and PV $(277,300)$ as well as haematocrit (Hct) and $[\mathrm{Hb}]$ have been reported $(61,287)$. For instance, Fortney et al. (86) calculated relative \% changes in PV from daily measurements of $[\mathrm{Hb}]$ and Hct in 8 healthy females (20$35 \mathrm{yr}$ ). They observed a large transient increase in PV within $2 \mathrm{~d}$ of ovulation, which was followed by a 1-3 d decrease in PV. During the luteal phase, they noted a progressive ( 200 
$\mathrm{ml}$ ) increase in PV, which peaked $\sim 2-3 \mathrm{~d}$ prior to menses. In agreement, Turner et al. (277) observed PV peaks near ovulation as well as expansion later in the luteal phase (10-13 d post ovulation). Moreover, the luteal phase has been associated with a decrease in [Hb] and Hct compared to the follicular phase $(61,287)$, which agrees with hemodilution occuring at this time. However, Dombovy et al. (61) postulated that during the luteal phase, the decrease in $[\mathrm{Hb}]$ and the expected rightward shift of the oxyhaemoglobin disassociation curve caused by a higher body temperature $\left(-0.5^{\circ} \mathrm{C}\right)$, in addition to the expected leftward shift caused by an increase in $\mathrm{V}_{\mathrm{E}}$ and lower $\mathrm{PCO}_{2}$, results in no net effect on oxygen delivery to the tissues. In contrast, Lebrun (162) observed no differences in either [Hb] or Hct during the luteal phase compared to the follicular phase.

Greater circulating $\mathrm{ADH}$ levels have been observed at ovulation and are associated with increased levels of estrogen, while greater concentrations of renin, angiotensin and aldosterone have been observed in the luteal phase and are associated with increased levels of progesterone $(55,56,96,263)$. Increased blood estrogen levels, which occur $\sim 2 \mathrm{~d}$ prior to ovulation and to a lesser extent during the luteal phase for a more prolonged time, have been associated with fluid retention (86). However, it has been postulated that progesterone must be low to see this effect (87). Increased progesterone levels which occur during this phase act at the kidney to antagonize the action of aldosterone, thereby resulting in a loss of sodium and water. The loss of sodium as well as the increase in norepinephrine observed at this time (263) stimulate the renin-angiotensin system and produce an exaggerated increase in the secretion of aldosterone, as well as the release of $\mathrm{ADH}(56)$. These hormones are 
responsible for sweat sodium and chloride retention and renal sodium retention (298).

However, a common effect of strenuous training on the menstrual cycle is a shortened duration of the luteal phase (146) associated with lower concentrations of progesterone (249). In line with this, some ET compared to NA females display lower levels of aldosterone during the midluteal phase (249). Wilkerson et al. (300) observed significant increases in PV and total plasma protein concentration during the luteal phase in females with normal progesterone levels, but not in those with low progesterone. It was concluded that females with regular menstrual cycles but without normal luteal increases in progesterone, may not display the same haematological changes between menstrual cycle phases as would females with normal luteal progesterone (300). These authors suggested that when haematological investigations on females are performed, not only the menstrual cycle phase, but also the hormonal status should also be considered.

The increased progesterone, and thus aldosterone persists through exercise in the luteal phase, and as such, may have the potential to influence PV shifts during exercise (55). Some investigators have observed more rapid and greater vascular fluid shifts during exercise in the follicular phase (263) along with a more rapid haemoconcentration compared to the luteal phase (96). In contrast, Stephensen et al. (263), reported that by 3 min into exercise, the amount of fluid shifted was independent of menstrual cycle phase. In agreement with the latter, Fortney et al. (89) observed no menstrual phase differences in haemoconcen-tration during cycle ergometer exercise. When DeSouza et al. (55) tested eumenorrheic and amenorrheic female runners (18-37 yr) they observed significant increases in plasma ADH, 
and renin during submaximal exercise, independent of menstrual phase and status. Thus, it appears that menstrual phase and status may not have a significant influence on PV shifts during exercise.

It has been postulated that the estrogen-induced increase in PV from OCA could have a beneficial effect on performance by enhancing preload. Lehtovirta et al. (164) examined the effects of a combined OCA on the hemodynamics in 14 healthy females (19-24 yr). They observed a significant increase in BV after 2 mo of OCA use. However, Lebrun (163) observed no difference in $[\mathrm{Hb}]$ in low dose OCA users compared to a placebo group. It has been postulated that when haematology examination is conducted during the same phase of the menstrual cycle, (ie. follicular phase) hormonal levels of OCA users and non-users do not differ significantly from one another, and thus [Hb], Hct, PV and BV should also not differ. ii) Influence of menopause and HRT

Stevenson et al. (265) reported no differences in BV, PV or RCV in ET females who were using HRT compared to those who were not. In agreement, Bush et al. (21) examined active female HRT users and non-users (48-60 yr) and concluded that HRT does not influence exercise-induced PV shifts in post-menopausal females. Thus, more investigations need to be conducted before any conclusions can be drawn regarding the effect of OCA and HRT on haematology, and on cardiovascular and thermoregulatory responses during exercise.

In comparison to males, circulating levels of aldosterone were reported to be two times larger in females who were examined during the luteal phase, with no gender differences observed in the follicular phase (112). It has been observed that males and females have 
similar PV responses during exercise when females are examined during the follicular phase of the menstrual cycle (96). Although postmenopausal females have decreased levels of both progesterone and estrogen $\left(10-30 \mathrm{pg} \cdot \mathrm{ml}^{-1}\right)$, normally menstruating females display similar levels of these hormones while they are in the early follicular phase (271). Estrogen replacement therapy in postmenopausal females, increases estrogen to a level which is similar to that observed in the late follicular phase in regularly menstruating females (271). Thus, most physiological and clinical research on females is conducted during the follicular phase of the menstrual cycle.

\section{Age-Related Changes in BV}

Investigators have reported that $\mathrm{BV}$ in older males is lower (53) or similar (26) to younger males. In four recent investigations, Evan's blue dye-dilution was used to measure PV in older and younger individuals. For instance, when Davy and Seals (53) compared males in their sixties with males in their twenties matched for being healthy and untrained, they observed a lower BV and PV in the older males compared to the younger males (63 vs $83 \mathrm{ml} \cdot \mathrm{kg}^{-1}$ and $39 \mathrm{vs} 50 \mathrm{ml} \cdot \mathrm{kg}^{-1}$, respectively). In a longitudinal investigation of males and females $(60-80 \mathrm{yr}), \mathrm{VO}_{2}$ max was increased by $12 \%$ after 6 mo of endurance training and $\mathrm{BV}$, PV and RCV was expanded by 12,11 and $14 \%$ respectively (24). It was concluded that the capacity to increase PV and thus, BV with endurance training is maintained in the healthy elderly. Similarly, Jones et al. (136) observed similar high BV in ET pre-menopausal (29 yr) and ET post-menopausal (62 yr) females ( 85.2 and $84.7 \mathrm{ml} \cdot \mathrm{kg}^{-1}$, respectively). Plasma volume and RCV were also similar between groups. The authors concluded that BV is 
maintained with age in ET females. This suggests that high levels of physical activity may prevent the decline in BV which has previously been observed with advancing age in sedentary individuals.

Since BV is influenced by body size, LBM and physical activity level, which all tend to decline as a function of age, one or more of these factors may account for the reductions observed in BV in sedenatary individuals. For instance, although Chien et al. (26) reported that BV was maintained over $17 \mathrm{yr}$ in males ( $<50 \mathrm{yr}$ ), when adjustment was made for the increase in body mass over the same period of time, BV actually declined by $-10 \%$. Convertino et al. (38) noted that after $10 \mathrm{~d}$ of bed rest in middle-aged males, PV decreased 10-15\%. Stevenson et al. (265) showed that $\mathrm{VO}_{2} \max$ and $\mathrm{BV}$ were directly correlated in postmenopausal women who differed in their levels of physical activity. Thus, it was proposed that PV is strongly influenced by physical-activity and only secondarily influenced by age (53). In summary, a larger BV has been observed in ET compared to NA individuals. An augmented BV improves endurance performance via enhancements in both cardiovascular and thermoregulatory mechanisms during exercise. When expressed relative to body mass, BV is independent of both gender and age in ET individuals.

\section{Diastolic Filling Rate in ET Individuals and the Relationship to Gender and Age}

Recent research has shown that, in contrast to the classical explanation of an enhanced systolic function being the major determinant of an augmented cardiac function, an enhanced diastolic function plays a larger role $(105,186)$. In ET males, cardiac function is enhanced primarily due to an increased DFR $(70,105,152,186,289)$, with LVER being relatively 
unchanged $(11,72,79,166,279)$.

Diastolic filling consists of four phases 1) isovolumic relaxation, 2) a rapid filling period, 3) diastasis (slow filling period) and 4) atrial systole (186). Isovolumic relaxation consists of the period after aortic valve closure and before the ventricular pressure drops below the atrial pressure. As soon as the ventricular pressure drops below the atrial pressure, the mitral valve opens and the rapid filling period begins, accounting for $\sim 80 \%$ of ventricular filling (186). Following this rapid filling phase is a slow filling phase (diastasis), which accounts for $<5 \%$ of ventricular filling. Atrial systole is the final phase of diastole and accounts for $\sim 15 \%$ of ventricular filling. During exercise, high HR (ie, $>170 \mathrm{bpm}$ ) are thought to reduce or eliminate diastasis, such that the rapid filling period can be maintained (186). It has been postulated that the enhanced early DFR and the faster rate of relaxation (during diastasis) will allow for a more complete filling of the ventricle during the later stages of strenuous exercise when DT shortens at high HR $(105,152,167,186,289)$.

Diastolic function can be measured noninvasively by radionuclide techniques, echocardiography and CTI $(105,186)$. Thus, caution must be taken when comparing results from investigations which use different measurement techniques.

\section{Diastolic Filling Rate in ET Compared to NA Individuals}

Controversy exists regarding the endurance training related adaptations in the diastolic function. While some investigators have observed no differences in diastolic function of ET compared to NA males $(72,81,111,243,250)$, others have shown that both younger and older ET males exhibit an enhanced diastolic function at rest and during exercise compared 
to age-matched NA males $(33,63,71,79,85,167,186,256)$. For instance, Matsuda et al. (186) examined male middle and long-distance runners (18-22 yr) and NA males (19-26 yr) via M-mode echocardiography. They observed an enhanced early DFR at rest and during submaximal exercise (100 bpm) in runners compared to NA males. By measuring CTI and SV simultaneously, two recent investigators have observed that ET younger males have augmented DFR (SV/DT) and LVER (SV/LVET), with the primary advantage of ET over NA males in the DFR $(105,152)$. For instance, at DFRmax and LVERmax were $\sim 71 \%$ and 20\% larger, respectively, in ET younger males compared to NA age-matched males (105). Mechanisms Responsible for the Enhanced DFR in ET Compared to NA Individuals

Diastolic filling may be enhanced via: 1) an increased venous return and thus, preload (which depends upon BV) $(79,85,152,186,289), 2)$ an enhanced rate and extent of ventricular relaxation (which depends upon myocardial compliance) $(33,71,186)$, and/or 3$)$ an increased pressure gradient between the left ventricle and left atrium, immediately prior to mitral valve opening $(166,167,278)$.

i) Breload

Krip et al. (152), extended the work of Gledhill et al. (105) and measured the effect that BV has on the cardiac function of ET and NA males. Similar to Gledhill et al. (105), they observed a significantly larger Qmax, SVmax, DFRmax and LVERmax, along with a progressive increase in SV throughout incremental exercise to maximum, in ET compared to NA males. Immediately following a $500 \mathrm{ml}$ reduction in BV in ET males, Qmax, SVmax, $\mathrm{VO}_{2}$ max and DFRmax decreased significantly. While in NA males, a $500 \mathrm{ml}$ increase in BV 
resulted in significant increases in these four variables. The authors concluded that a large portion of the differences in cardiac function between ET and NA males are the result of passive responses to an augmented BV in ET males, which in turn favours adequate LV filling when DT shortens at high HR, thus enabling SV to continue to increase up to maximum exercise via the Frank-Starling mechanism. Although LVER was also related to BV, the impact was not as large. It was proposed that the enhanced LVER is a passive response to an elevated EDV consequent to an enhanced DFR and the resultant effects of the FrankStarling mechanism (152).

Extending the work of Krip et al. (152), Warbuton et al. (289) increased the BV (by $500 \mathrm{ml}$ ) of elite male cyclists, who already had a baseline augmentation in BV. These authors observed that although Qmax and SVmax were significantly larger after BV expansion, there were no effects on $\mathrm{VO}_{2} \max$, DFRmax or LVERmax. These authors postulated that ET athletes may already be at an optimal BV which is at the limit of their diastolic reserve capacity. In agreement, other investigators have proposed that a diastolic reserve capacity exists and is recruited in response to optimal increases in BV, beyond which no further increases in SV will occur in either ET or NA individuals $(45,128)$. Since physiological LV hypertrophy has been associated with improved indices of diastolic filling at rest and during exercise in both younger and older ET males $(33,62,72,79,85,167)$, it is possible that this mechanism may be partly responsible for setting the upper limit of diastolic reserve capacity.

\section{ii) Left ventricular relaxation}

An increase in the rate and extent of LV relaxation during exercise allows for an 
enhanced early DFR. In ET individuals, an enhanced rate of relaxation has been attributed to an augmented elastic recoil, which results from a decreased ESV, in association with an increased negative LV pressure $(25,186,315)$. In addition, during exercise, increased catecholamine secretion has been observed to enhance LV relaxation in both animals and humans (278). Evidence for this comes from an investigation showing a decrease in peak DFR at all exercise intensities in younger individuals who were given propranolol ( $\beta$-AR blockade) (243). Endurance training has also been shown to enhance the compliance and distensibility of the myocardium, in turn resulting in an elevated DFR, and thus SV (165).

\section{iii) Bressure gradient}

An increased pressure gradient between the LV and left atrium (by either an elevation in left atrial pressure or a reduction in LV pressure), immediately prior to mitral valve opening, increases flow velocity across the mitral valve and thus, enhances early DFR (167). Mechanisms responsible for an increase in left atrial pressure in ET individuals are: an enhanced central venous pressure (due to an augmented BV) $(167,278)$, a more vigourous atrial contraction (186) and/or an increase in pulmonary capillary wedge pressure via an increase in pulmonary venous flow (148). Mechanisms responsible for a reduction in LV pressure are: a more complete emptying of the LV (278), which may result from a filling pressure induced dilation of the $\operatorname{LV}(25,29)$ and/or enhanced inotropic stimulation. For instance, in ET compared to NA individuals, an increased peak rate of lengthening of the LV, which results from an increased peak velocity of LV filling (due to an augmented venous return), leads to an increased rate of LV shortening, and a more complete emptying of the 
LV. This increased velocity of shortening enhances the rate of decrease in LV pressure during isovolumic relaxation, thus increasing the early DFR in ET compared to NA individuals (186). The LV pressure may continue to fall (despite the increase in LV volume), creating a suction effect, or "self-enlargement" of the LV, thus drawing blood from the atrium $(14,186,232)$. The suction effect may be augmented by the actions of sympathetic and $\beta-A R$ stimulation $(25,243,278)$, which in turn, enhances myocardial relaxation, thus increasing the rate and extent of $L V$ pressure decay to develop very low or even negative early diastolic pressures $(-1$ to $-3 \mathrm{mmHg})(278)$. This allows the $\mathrm{LV}$ to fill to significantly larger volumes during early diastole, despite the marked reduction in DT that occurs at higher HR. Matsuda et al. (186) postulated that the increased DFR in ET individuals may be an adaptation to repeated exposure of chronically elevated HR that are characteristic of endurance training.

\section{Gender Differences in DFR}

As previously discussed, females may rely to a greater extent upon the Frank-Starling mechanism to augment SVmax, compared to males who rely upon changes in $\mathrm{EF}(1,81,118$, 125). Fagard et al. (72) used imaging and Doppler echocardiography and observed that despite the significantly larger LV masses and LVEDD in younger female distance runners, the early DFR (estimated from the velocity of LV relaxation and LV inflow pattern) was similar to that of age-matched control females. In addition, the unchanged ratio of peak velocities of LV filling during atrial systole and early filling, suggests that LV distensibility was unaltered in the female athletes. It was concluded that, in contrast to ET males $(33,62$, $63,72,79,85,167)$, physiological left ventricular hypertrophy in ET females is not associated 
with improved indices of diastolic filling at rest or during exercise.

Spina et al (256) examined sedentary males ( $66 \pm 1 \mathrm{yr})$ and females $(64 \pm 1 \mathrm{yr})$ who underwent an endurance training program ( $3 \mathrm{mo}, 50 \mathrm{~min} \cdot \mathrm{d}^{-1}, 3-4 \mathrm{~d} \cdot \mathrm{wk} \mathrm{k}^{-1}$ at $80 \%$ of $\mathrm{HRmax}$ ). Although the older males showed an increase in resting and peak LV filling rates (counts $\cdot \mathrm{s}^{-1}$ ), and a decrease in the time to peak filling rate (TPFR), the older females did not display these training-induced adaptations. The authors postulated that one reason for this gender difference could be the fact that the females had a faster pre-training TPFR than the males.

\section{Age-Related Changes in DFR}

The DFR has been shown to decrease as a function of age at rest and during exercise in sedentary individuals via pulsed Doppler transmitral flow velocity profile $(18,148,237$, 261), M-mode echocardiography $(97,101)$ and radionuclide ventriculography $(4,12,193)$. The peak rate at which the LV fills with blood during early diastole is reduced by $\sim 50 \%$ between ages 20-80 yr in healthy males and females $(12,101,148)$. However, this reduction in early DFR does not result in a decreased EDV, since an augmented atrial contribution to LV filling compensates for the decreased early DFR (evidenced by an increased peak atrial filling rate and a decreased early to atrial (E/A) ratio of filling) $(18,196,237)$, such that EDV is maintained $(81,97,101)$. Thus, in contrast to younger individuals who rely on early DFR to augment SV, older individuals rely to a greater extent on late atrial filling $(4,261)$, which has been associated with left atrial enlargement in older males (101).

However, controversy exists regarding the age-related changes in DFR in ET individuals. While some investigators observed no difference in the DFR of masters males 
compared to sedentary age-matched males $(82,243)$, others showed an attenuation or reversal of this phenomenon with endurance training $(62,85,167,256)$. For instance, Fleg et al. (82) observed that older ET males (52-76 yr) demonstrated impaired early diastolic filling with compensatory increases in late atrial filling, compared to younger ET males ( $<40$ yr), and reported that this attenuation was similar in magnitude to that observed in sedentary age-matched males. By multiple regression analysis, they observed that age, but not treadmill $\mathrm{VO}_{2}$ max was a significant predictor of peak early filling velocity, peak late filling velocity and atrial filling fraction. In agreement, Schulman et al. (243) examined healthy sedentary males and masters males (22-82 yr). Peak filling rates at rest, 50\% of maximal exercise and maximal exercise correlated inversely with age $(r=-0.64,-0.53,-0.64$, respectively), with the decline in resting and exercise DFR in the masters males, being similar in magnitude to the sedentary age-matched males. Thus, the results of these two investigations suggest that the impairment of the early DFR is not secondary to a reduction in aerobic capacity that occurs with age.

However, in contrast, Takemoto et al. (270) examined older make distance runners (60 $\pm 1 \mathrm{yr})$ and sedentary age-matched males. In comparison to the sedentary males, the ET males had a decreased late peak filling velocity, and an increased E/A peak filling velocity. Forman et al. (85) observed exactly the same results when they examined younger (27-32 yr), and older sedentary males (58-68 yr) and masters males (52-66 yr). In addition, these authors observed that the pattern of LV filling in masters males was similar to that of younger sedentary males (85). Douglas and O'Toole (62), examined younger ( $23 \pm 2 \mathrm{yr})$ and older triathletes ( $58 \pm 6 \mathrm{yr})$ and sedentary younger $(25 \pm 3 \mathrm{yr})$ and older individuals $(65 \pm 6 \mathrm{yr})$. 
The two ET groups had similar lower resting HR, larger LVEDD at end-diastole, and higher E/A inflow velocities compared to the sedentary age-matched individuals. However the older athletes differed from the younger athletes by having higher SBP and DBP, greater posterior wall thickness, and lower E/A inflow velocity. It was concluded that the expected pattern of cardiac alteration normally seen in response to aging is modified in the older athlete, such that it resembles filling patterns more similar to younger sedentary individuals. Further, in response to $6 \mathrm{mo}$ of endurance training in previously sedentary younger (24-32 yr) and older (60-82 yr) males, Levy et al. (167) observed $14 \%$ increases in absolute peak DFR at rest and during exercise. In addition, training attenuated the increased resting atrial filling fraction and the peak atrial filling fraction (by $27 \%$ ) in the older males, while no changes in these variables were observed in the younger males. Univariate and multivariate analyses showed that the DFR at rest and during exercise was highly correlated with $\mathrm{VO}_{2} \max$. These authors postulated that the augmentation in DFR may be an important adaptation which allows Qmax, $\mathrm{SV} \max$, and $\mathrm{VO}_{2} \max$ to be increased during exercise. Further, the results of these investigations suggest that endurance training may slow or even reverse the age-related changes in LV filling dynamics, thereby preserving filling patterns more similar to younger individuals.

\section{Mechanisms Responsible for the Age-Related Changes in DFR}

i) Mechanisms responsible for an age-related decline in DER

Mechanisms that may be responsible for the decline in DFR are: 1) prolonged contraction and relaxation times, 2) decreased diastolic compliance and 3) decreased 
responsiveness to $\beta$-AR stimulation (156). A prolonged contraction time results from a slower time $(-15-20 \%)$ to peak tension and a longer time for relaxation after peak tension is obtained $\left(\mathrm{RT}_{1 / 2}\right)$, which in turn, allows some of the contractile activity to persist at the time of mitral valve opening, thus delaying the early LV filling $(262,272)$. Isovolumic myocardial relaxation time (time between aortic valve closure and mitral valve opening), has been shown to become prolonged $(\sim 40 \%)$, with aging in both males and females $(120,261)$. A prolonged relaxation is associated with a decreased rate of $\mathrm{Ca}^{2+}$ transport and re-uptake by the cardiac sarcoplasmic reticulum (SR), following myocardial contraction in rats $(273,159)$. The slower rate of $\mathrm{Ca}^{2+}$ transport has been shown to be due to a decreased content of the SR Ca-ATPase protein, (269). It has been postulated that the molecular mechanism underlying this change may be an alteration of the gene (SERCA2a) transcription, since the steady state mRNA of the gene is decreased by $40-50 \%$ in the RNA isolated from old rats (269).

Additionally, there is an age-related shift in the contractile protein myosin from a fast (alpha-myosin) to a slow ( $\beta$-myosin), resulting in a slowing of the actin-activated myosin ATPase, and a decreased peak velocity of shortening in rats (156). Further, although increased ventricular stiffness has been associated with delayed relaxation at end-diastole and thus, a decreased early DFR in older rats (201), it does not correlate with peak early DFR in humans (176). Lastly, an age-related reduction in the response to $\beta$-adrenergic stimulation likely contributes to the impairment in both peak DFR and systolic contractile performance $(243,245,266)$. For instance, in response to propranolol ( $\beta$-AR blocker), Schulman et al. (243), observed a decrease in exercise peak filling rate in younger sedentary males ( $37 \pm 8 \mathrm{yr}$ ), 
but not in older sedentary or masters males $(62 \pm 6 \mathrm{yr})$. They concluded that the decline in DFR with age appears to be related to a decrease in $\beta$-AR responsiveness in older individuals. ii) Mechanisms responsible for an attenuated age-related decline in DER in ET compared to NA individuals

It has been postulated that the mechanism responsible for the attenuated decrease in the age-related decline in resting and exercise DFR in ET individuals is an enhanced rate of relaxation. The mechanism responsible for an enhanced rate of relaxation in response to endurance training initiated in old age in rats, was an increase in the $\mathrm{Ca}^{2+}$ uptake and transport by the SR, due to an upregulation of the SR Ca-ATPase protein $(262,273)$. Although, it appears that the isoformic shift in myosin (decrease in mRNA alpha-myosin and an increase in mRNA $\beta$-myosin) cannot be changed by exercise training initiated in old age (273). However, in response to endurance training in male rats at weaning, the aging-related isoformic shift of myosin appeared to be blocked, suggesting that the peak velocity of shortening in the myocardium may have been preserved (273). Thus, although the early DFR decreases at rest and during exercise with aging, endurance training may prevent some of this decline. However, further research is needed before any conclusions may be drawn.

In summary, ET individuals have a enhanced DFR compared to NA individuals primarily due to an augmented BV and thus preload. However, the literature is controvesial with regards to the age-related changes in DFR of ET individuals. Thus, more investigations are required before conclusions may drawn about older ET individuals. Further, since only two investigators to date have examined the DFR in ET females, more research is needed in 
this area before any conclusions may be drawn. 


\section{SUMMARY OF LITERATURE REVIEW}

\section{Endurance Training}

Endurance trained, compared to NA males have a significantly larger $\mathrm{VO}_{2} \max$, which results from their greater $\mathrm{Qmax}$, and a- $-\mathrm{vDO}_{2} \max$. Since $\mathrm{HRmax}$ does not change with training, the elevated Qmax results from an augmented SVmax. Both a larger LVEDV and a smaller LVESV contribute to the enhanced SVmax. An elevated LVEDV results primarily from an enhanced preload, due to an elevated BV in ET compared to NA males. A decreased LVESV may result from increases in myocardial contractility, although in ET males the primary mechanism responsible for a smaller LVESV is a decreased afterload during maximal exercise, compared to NA males. Endurance trained males also have a larger a-viDO ${ }_{2} \max$ compared to NA males, although in both younger and older males an elevated Qmax contributes more to the increased $\mathrm{VO}_{2} \mathrm{max}$. Further, ET males display a faster DFRmax compared to NA males. Investigators have recently shown that the primary mechanism responsible for the increased DFRmax is an augmented BV, which in turn favors adequate ventricular filling when DT shortens at higher HR. Furthermore, investigators have observed recently that in contrast to the classical explanation of a plateau in SV at submaximal HR, the influence of an augmented BV on the DFRmax of ET males, enables SV to continue to increase up to maximum, without a plateau, via the Frank-Starling mechanism.

\section{Gender}

Endurance trained females also display a significantly larger $\mathrm{VO}_{2} \max$, due to a greater Qmax, SVmax and a- $-\mathrm{vDO}_{2} \max$ compared to NA females. However, controversy exists 
regarding the primary mechanisms responsible for the enhanced SVmax. Similar to males, ET females display training-induced augmentations in BV, and thus have an elevated LVEDV from an enhanced preload. Since few investigations exist to date regarding the traininginduced changes in LVESV in females, more research is needed in this area before conclusions may be drawn. Endurance trained females also have a larger $\mathrm{a}-\overline{\mathrm{v}} \mathrm{OO}_{2} \max$ compared to NA females. Also, in comparison to males, an elevated a- $\bar{v} \mathrm{DO}_{2} \max$ in younger and older females may contribute more to the increased $\mathrm{VO}_{2} \max$ than an elevated $\mathrm{Qmax}$. However, more research is needed in this area. Further, although ET males display an elevated DFRmax compared to NA individuais, this has not yet been observed in the few studies to date in which ET females are examined. Since both younger and older ET females have an elevated BV compared to NA females, they should also display an augmented DFRmax. However, to date no research exists in which the SV responses of females during incremental exercise to maximum has been examined, and thus it is unknown wether they display similar training-induced increases in SV up to maximum, without a plateau, as previously observed in ET males.

\section{Age}

Although $\mathrm{VO}_{2}$ max declines progressively with advancing age, ET males and females show an attenuation of this decline. However, controversy exists regarding the mechanisms responsible for this attenuation. While some investigators show a maintenance of $\mathrm{Qmax}$, due to an augmented SVmax, more have observed a decline in Qmax, resulting from decreases in both SVmax and HRmax or solely due to a decrease in HRmax. However, ET males and 
females appear to be able to maintain their a- $\overline{\mathrm{vDO}} \mathrm{O}_{2} \max$ with aging. A decline in $\mathrm{SVmax}$ has been shown to result primarily from an increased LVESV, since the increased LVEDV is maintained or even augmented with aging. A larger LVESV results from an age-related decline in myocardial contractility, as well as from an age-related increase in afterload. However, controversy exists regarding the extent to which these two age-related changes may be attenuated with endurance training. Further, although ET males and females are able to maintain their BV with aging, controversy exists regarding the age-related changes in DFRmax. While some investigators have shown that, similar to NA males, ET males have a decreased DFRmax, others have observed an attenuated decline in the DFRmax with endurance training. Thus, more research is required in this area before conclusions may be drawn. The one investigator to date who examined older ET males throughout incremental exercise to maximum, revealed a progressive increase (in 5 out of 11 masters male runners), in SV up to maximum without a plateau (222). No studies have been conducted to date on ET females, and thus investigation is required in this area, before conclusions may be drawn. 


\section{CHAPTER III \\ METHODS AND PROCEDURES}

\section{Introduction}

This chapter contains an explanation of the experimental methodology and testing procedures applied in this investigation.

\section{Participants}

Twenty-three female participants, 20 to $63 \mathrm{yr}$, participated in this study with informed consent (Appendix F) and approval of the York University Human Participants Committee. Participants were either cyclists, marathon runners, duathletes, or triathletes and had participated in regular endurance training for a minimum of five yr. Each participant was screened for a high aerobic capacity, and a normal forced vital capacity (FVC) and was then assigned to one of four groups based on their age. Group 20 participants were 20-29 yr $(\mathrm{n}=6)$ and possessed a $\mathrm{VO}_{2} \max$ greater than $55 \mathrm{ml} \cdot \mathrm{kg}^{-1} \cdot \mathrm{min}^{-1}$, group 40 participants were 40 $45 \mathrm{yr}(\mathrm{n}=6)$ and exhibited a $\mathrm{VO}_{2} \max$ in excess of $45 \mathrm{ml} \cdot \mathrm{kg}^{-1} \cdot \mathrm{min}^{-1}$, group 50 participants were 49-54 yr $(\mathrm{n}=6)$ and had a $\mathrm{VO}_{2} \max$ not less than $41 \mathrm{ml} \cdot \mathrm{kg}^{-1} \cdot \mathrm{min}^{-1}$ and group 60 participants were 58-63 yr $(\mathrm{n}=5)$ and possesed a $\mathrm{VO}_{2} \max$ greater than $40 \mathrm{ml} \cdot \mathrm{kg}^{-1} \cdot \mathrm{min}^{-1}$. The four participant groups were matched for lean body mass.

\section{Experimental Design}

A mixed model experimental design was employed with four groups of ET females. The a- $\mathrm{DDO}_{2}, \mathrm{Q}$ SV, CTI (LVET, PEP, DT), DFR, LVER, BP, MAP and TPR measurements were compared between groups at rest and at identical target HR ( $\pm 1 \mathrm{bpm}$ ) of 110,130,150, 
$170 \mathrm{bpm}$ and max. In addition, BV, [Hb], Hct, and anthropometric measurements (body mass, LBM, height) were determined and compared between groups.

\section{Test Protocol}

All testing was conducted at the York University Human Performance Laboratory. Premenopausal participants were studied during the follicular phase of their menstrual cycle (1 to 8 days post menses) in an attempt to control for the effects PV shifts which occur during the menstrual cycle. Participants reported to the laboratory on four occasions. Prior to initiating data collection, on the first day, participants were screened for a high aerobic capacity (in the appropriate range based on their age) and a normal FVC ( $\geq 80 \%$ of normal predicted values). The work rates that elicited $H R$ of $110,130,150,170 \mathrm{bpm}$ and max were determined for each participant. The measurements of $\mathrm{VO}_{2}$, CTI, and BP were conducted at rest and at each target HR. In addition, participants were familiarized with the acetylene rebreathe manoeuvre to measure $Q$ at each $H R$. Maximal oxygen uptake was assessed via the open circuit technique on an electrically braked cycle ergometer (Warren E. Collins Inc., Braintree, MASS). A supramaximum work rate was performed by each participant to confirm that both $\mathrm{VO}_{2} \max$ and $\mathrm{HRmax}$ were achieved (274). In preparation for the second visit to the laboratory, participants were instructed to arrive in a fasted ( $12 \mathrm{~h})$ and rested state and to have refrained from heavy training and the consumption of alcohol and caffeine $24 \mathrm{~h}$ prior to the test.

On the second day, participants had their BV measured using Evan's blue dye in a standard laboratory dilution technique (26). In addition, they were given a second 
opportunity to practice the acetylene rebreathe manoeuvre at rest and at each target HR. In preparation for the final two days of the study, participants were again instructed to refrain from heavy training and the consumption of alcohol and caffeine $24 \mathrm{~h}$ prior to each test session. They were also instructed to drink an amount of water equal to $1 \%$ of their body weight on the nights before each test, and $300 \mathrm{ml}$ on the morning of each test to ensure adequate and uniform hydration.

Upon arrival to the laboratory, on days three and four, participants had their body mass measured and on one of the two days, they had their LBM determined via subcutaneous skinfold measurements. Blood samples were taken prior to exercise on both days for the determination of $[\mathrm{Hb}]$ and $\mathrm{Hct}$ to monitor changes in control BV (19). During the cycling exercise, measurements of $\mathrm{VO}_{2}, \mathrm{CTI}, \mathrm{BP}$, and $\mathrm{Q}$ were taken at rest and during $\mathrm{HR}( \pm 1 \mathrm{bpm}$ ) of $110,130,150,170 \mathrm{bpm}$ and max. The duration of exercise at each incremental work rate was $4 \mathrm{~min}$, with simultaneous recordings of $\mathrm{VO}_{2}$ and CTI conducted $90 \mathrm{~s}$ into each exercise stage, followed by the determination of BP. This provided ample time in the first $90 \mathrm{~s}$ of exercise at each successive HR to achieve a steady state before measurements were taken. Duplicate acetylene rebreathe manoeuvres for the determination of $Q$ were recorded in the final 2 min. At least $45 \mathrm{~s}$ separated each rebreathe manoeuvre to allow for complete elimination of acetylene gas. The values reported were an average of the measurements taken on days three and four.

\section{Determination of Heart Rate}

An ECG was recorded during all data collection and HR was monitored continuously 
by a cardiotachometer (Polar HR monitor). The HR monitor consisted of a removable transmitter which was affixed to two permanent electrodes built into an adjustable strap. A watch/ receiver converted the signals from the transmitter to digital readouts for HR. Two receivers were attached to the cycle ergometer, one for the experimenter and one for the participant to monitor her own HR. To ensure accurate readings were given by the HR monitors, the readouts were validated by an ECG (Sensor Medics, Anaheim, CA) attached to the participant. The ECG was recorded with silver-silver chloride electrodes filled with conductive gel (Medical Systems Corp., Greenvale, NY). Electrodes were attached to the participant with double adhesive collars and adhesive tape in a lead II configuration. The reference (positive) electrode was placed on the right side, over the manubrium-sternum, and the exploring (negative) and ground electrodes were placed over the fifth intercostal space, in the left and right anterior axillary lines respectively. To improve signal conductivity, rubbing alcohol was used to clean the three landmark sites. The wires from the electrodes were led around to the subject's right side, where they were secured with adhesive tape to minimize noise artifact. HR was recorded on a two-channel linear chart recorder (Watanabe Instruments Corp., Model WR 3001 Mark V, Tokyo, Japan) at a paper speed of $25 \mathrm{~mm} \cdot \mathrm{s}^{-1}$. HR at each workload was calculated from the number of R waves observed on the ECG in $10 \mathrm{~s}$. The coefficient of variation for the attainment of the target HR during submaximal exercise was $1.0 \%$ and during maximal exercise was $0.9 \%$ in all four groups.

\section{Determination of Oxygen Uptake}

Oxygen uptake was measured $90 \mathrm{~s}$ into each exercise stage via the open circuit 
technique. Participants wore a nose clip and breathed ambient air through a rubber mouthpiece attached to a two-way, low resistance and low dead space breathing valve (Ewald Koegel Co., San Antonio, TX). To prevent air leakage through relaxed lips during resting measurements, participants breathed through a face mask with a two-way breathing valve attached (Speak-Easy Face Mask, Hans Rudolph Inc., Kansas City, MO), which sealed their nose and mouth. Expired gas was directed through a flexible plastic hose $(3.8 \mathrm{~cm}$ wide) to a 1201 chain-compensated Tissot gasometer tank (Warren E. Collins Ltd., Braintree, MASS). Prior to each gas collection, the hose and gasometer were flushed with the participant's expired air for 2 to $3 \mathrm{~min}$ at rest and approximately $20 \mathrm{~s}$ during exercise, to replace the dead space (about $4 \mathrm{l}$ ) containing expired air from the previous workload. The temperature of the expired gas was read from a thermometer on the gasometer during each gas collection.

Following each gas collection, expired air was analyzed for the fractional concentrations of oxygen $\left(\mathrm{F}_{\mathrm{E}} \mathrm{O}_{2}\right)$ and carbon dioxide $\left(\mathrm{F}_{\mathrm{E}} \mathrm{CO}_{2}\right)$ using rapid response oxygen and carbon dioxide analyzers (Applied Electrochemistry Inc., Model S-3A, Sunnyvale, CA and Dwyer Instruments Inc., Model 17515, Michigan City, IND, respectively). Before reaching the analyzers, the sampled gas was drawn through a granular drying agent ("Drierite" - anhydrous $\mathrm{Ca} \mathrm{SO}_{4}$ ) so that the presence of water vapour would not effect the analysis. The analyzers were calibrated with a gas of known concentration, in the physiological range of measurement (Liquid Carbonic Inc., Toronto, ON) prior to the analysis of each expired air sample. The time of collection, volume of collected air and $\mathrm{F}_{\mathrm{E}} \mathrm{O}_{2}$ and $\mathrm{F}_{\mathrm{E}} \mathrm{CO}_{2}$ were recorded and entered into a computer pre-programmed with an equation used for the calculation of 
$\mathrm{VO}_{2}\left(\cdot \min ^{-1}\right)$ (see Appendix B). The participant's body mass in $\mathrm{kg}$ was used to calculate the participant's relative $\mathrm{VO}_{2}\left(\mathrm{ml} \cdot \mathrm{kg}^{-1} \cdot \mathrm{min}^{-1}\right)$. The coefficient of variation, expressed as the standard deviation divided by the mean difference between duplicate measurements of $\mathrm{VO}_{2}$ during submaximal exercise was $4.8 \%$ and during maximal exercise was $2.7 \%$ in all four groups.

\section{Determination of Cardiac Time Interyals}

The CTI measurements LVET, total electromechanical systole (Q-S $\mathrm{S}_{2}$, DT and PEP were recorded simultaneously, along with the $\mathrm{VO}_{2}$ measurements, at rest and $90 \mathrm{~s}$ into each exercise stage utilizing an ear pulse densitograph unit (EPD), (Hewlett Packard, Model 78016. Palo Alto, CA), a phonocardiogram (PCG) (Hewlett Packard, Model HP 21050A, Andover, MASS) and an ECG (Sensor Medics, Anaheim, CA).

i) Ear Pulse Densitography Measurement

An EPD is comprised of a photocell and a photoconductive element (cadmium selenide). The photocell is one of the branches of a Wheatstone bridge from which the output is fed into a pre-amplifier (Harvard Apparatus Models 354 and 371). After the signal was amplified, it was fed through a Beckman chart recorder (Type RM Dynograph $\circledast$ Recorder) with a built in $60 \mathrm{~Hz}$ filter, at a paper speed of $125 \mathrm{~mm} \cdot \mathrm{s}^{-1}$. The time constant was set to 0.3 ms, to provide a stable tracing during exercise, without affecting the CTI measurements.

The EPD was attached to the pinna of the participant's left ear. To improve signal conductivity, the participant's pinna was wiped with rubbing alcohol. The wire from the earpiece was looped over the participant's head and was held in place with an elastic, velcro- 
backed head-band Throughout the cardiac cycle, pulsatile changes in blood flow through the arterioles and capillaries of the ear produce proportional changes in the light (optical density) reaching the photocell (27) which are then recorded on chart paper.

ii) Phonocardiogram Measurements

First and second heart sounds ( $S_{1}$ and $S_{2}$, respectively) were recorded using a PCG. The PCG was placed over the participant's third or fourth intercostal space at the left sternal border. To improve signal conductivity, rubbing alcohol was applied to the landmark site. A perforated elastic strap ( 1 in. wide) was fastened around the participant's chest to secure the PCG. A mesh vest (SurgiVest, FRA Production ${ }^{\circledR}$ ) was worn to ensure contact between the skin and the PCG. After the signal was pre-amplified (Harvard Apparatus Models 354 and 371), it was fed through a two-channel linear chart recorder (Watanabe Instruments Corp., Model WR 3001 Mark V, Tokyo, Japan) with a built-in $60 \mathrm{~Hz}$ filter, at a naper speed of 100 $\mathrm{mm} \cdot \mathrm{s}^{-1}$. Heart rate and heart sounds were recorded simultaneously on the same chart recorder (Figure 1).

iii) Calculation of Cardiac Time Intervals

The derivation of CTI are shown in Figure 1. Left ventricular ejection time, which represents the phase of systole during which the left ventricle ejects blood into the arterial system, was determined from the EPD tracings. To ascertain the onset of LVET (when the aortic valve opens) two lines of extrapolation were drawn, one for the rapid upslope, and one for the baseline. A vertical line was drawn through the point of intersection of these two lines. The end point of LVET (aortic valve closure) was determined by first extrapolating the 

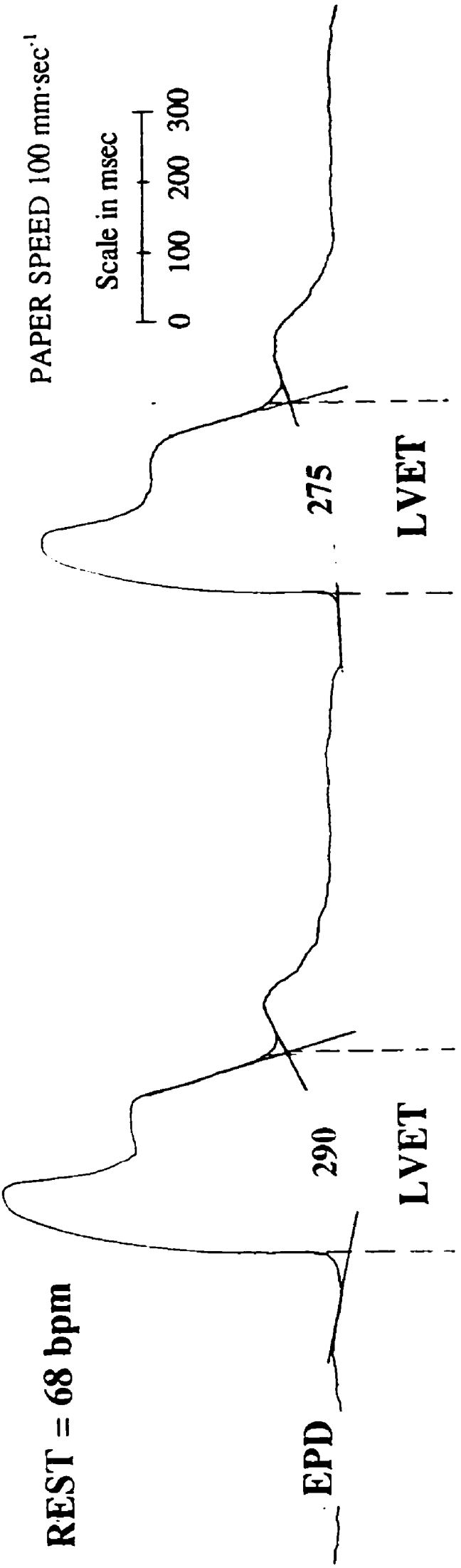

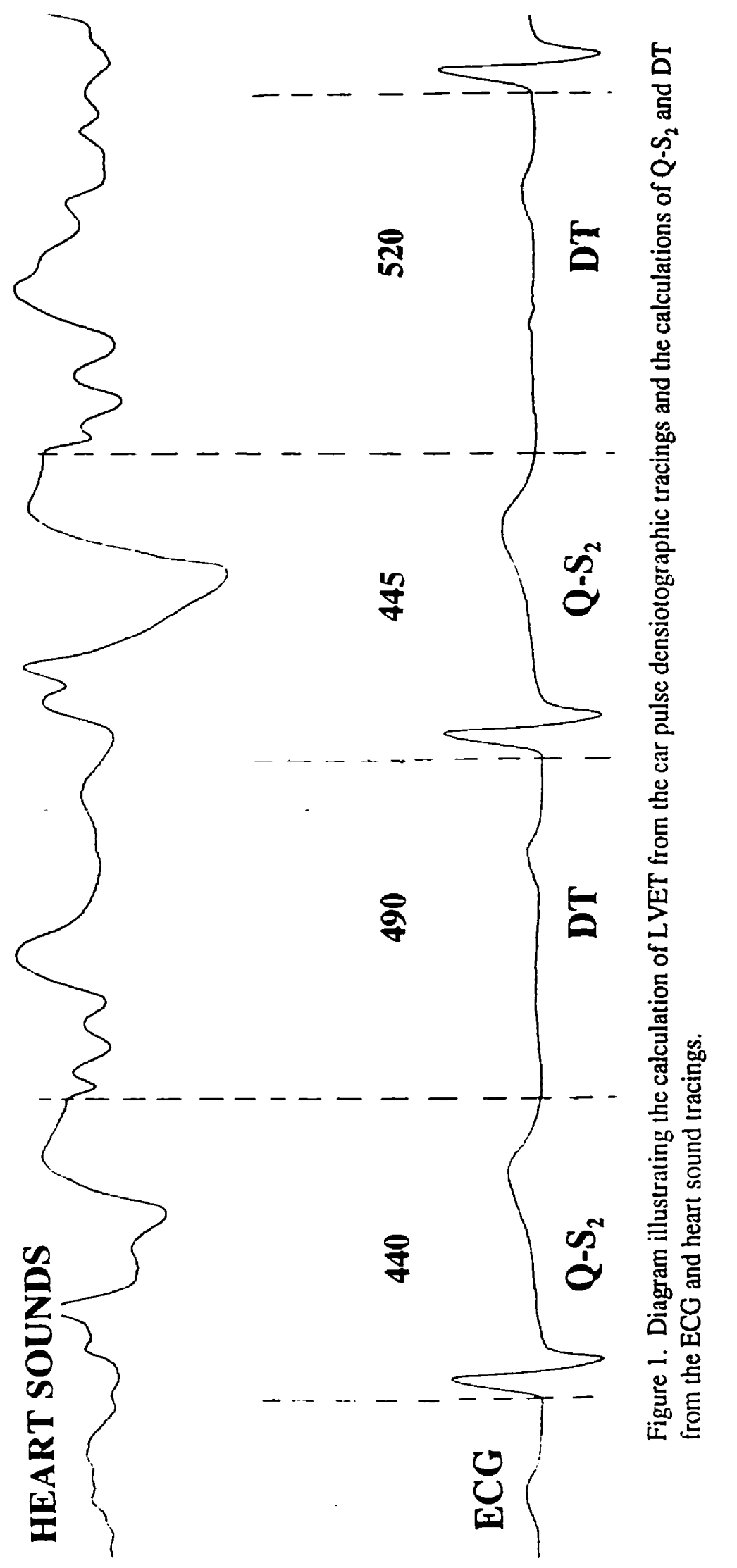


line of steep downslope until it intersected the dicrotic notch and then drawing a vertical line through the point of intersection. The distance between the two vertical lines was used to calculate the LVET interval. The Q- $S_{2}$ is comprised of PEP and LVET and was determined by measuring the time interval from the nadir of the $\mathrm{Q}$ wave on the ECG tracings to the initial high frequency vibration of $S_{2}$ (aortic valve closure) on the PCG tracings. The PEP was calculated by subtracting LVET from $\mathrm{Q}-\mathrm{S}_{2}$. The DT was determined by measuring the time interval from the peak of the initial high frequency vibration of $S_{2}$ on the PCG tracings to the nadir of the subsequent $Q$ wave on the ECG tracings (Figure 1).

During each of the $\mathrm{VO}_{2}$ measurements, a period of 10 to $20 \mathrm{~s}$ was used to obtain 10 consecutive cycles for the determination of CTI. From this sample, at least 5 out of the 10 cycles were sufficiently clear to allow for accurate CTI evaluation. The CTI were recorded to the nearest ms and were averaged over at least 5 cardiac cycles. The reduction and cakculation of all CTI data was performed by the same experimenter to control for the effects of inter-tester variability. In addition, a second experimenter periodically validated the results. The coefficient of variation expressed as the standard deviation divided by the mean difference between at least five determinations of CTI at rest and at each of the target HR was $3.9 \%$ at rest, $5.0 \%$ at $110 \mathrm{bpm}, 4.5 \%$ at $130 \mathrm{bpm}, 5.2 \%$ at $150 \mathrm{bpm}, 5.3 \%$ at $170 \mathrm{bpm}$ and 4.8 \% at HRmax in all four groups.

\section{Determination of Blood Pressure}

Blood pressure was measured using a sphygmomanometer and stethoscope applied to the participants left arm. Measurements were taken at rest and during each target $H R$, 
following the determination $\mathrm{VO}_{2}$. Mean arterial pressure $(\mathrm{mmHg})$ was calculated using the formula of DBP (mmHg) plus one-third pulse pressure (PP) $(\mathrm{mmHg})(\mathrm{MAP}=\mathrm{DBP}+1 / 3 \mathrm{PP})$ (where PP = SBP - DBP) at rest and at each target HR. Total peripheral resistance (mmHg. $\left.{ }^{1-1} \cdot \min ^{-1}\right)$ was determined from the ratio of MAP to $Q\left(1 \cdot \mathrm{min}^{-1}\right)(T P R=M A P / Q)$ at rest and at each target HR.

\section{Determination of Cardiac Output via the-Acetylene Rebreathe Method}

The acetylene rebreathe manoeuvre utilized in this study to determine $Q$ is a modification of the technique originally described by Grollman (116). A three-way breathing valve (Hans Rudolph Inc., Kansas City, MO) with a rubber mouthpiece attached was supended above the cycle ergometer. A 3 I latex rebreathing bag (Hans Rudolph Inc., Kansas City MO) was connected to the expiratory port and a 71 latex reservoir bag was connected to the inspiratory port. A plastic hose connected the reservoir bag to the gas cylinder which contained a gas mixture of $40 \% \mathrm{O}_{2}, 1 \%$ acetylene $\left(\mathrm{C}_{2} \mathrm{H}_{2}\right)$, and balance nitrogen $\left(\mathrm{N}_{2}\right)$ (Liquid Carbonic Inc., Toronto, ON). A pneumatically powered switching mechanism (Automated Controller, Model 4285B, Hans Rudolph Inc., Kansas City, MO) was used to change breathing ports. A small bore end-tidal sampling tube connected the breathing valve to the mass spectrometer (TC Centronics Ltd., Model 200 MGA, Croydon, ENG) for the continuous sampling of alveolar gas throughout the rebreathe manoeuvre.

To initiate the rebreathe manoeuvre, the participant rolled up the 31 latex bag to expel room air and with nose clips on, expired to residual volume (RV). The participant then inserted the mouthpiece and an experimenter switched open the inspiratory port. The 
participant then inhaled the gas mixture maximally. To ensure complete filling of the participant's lungs, the reservoir bag was continuously refilled with the gas mixture by an experimenter. When the participant's lungs were filled maximally, an experimenter switched open the expiratory port. The participant released the 31 latex bag and proceeded to rebreathe the gas mixture into and out of the bag, emptying and filling it with each breath, at a rate of one breath $\cdot \mathrm{s}^{-1}$, for an average of $8 \mathrm{~s}$ at rest and $5 \mathrm{~s}$ during heavy exercise. As the participant rebreathed from the bag, the expired gas mixture was continuously being sampled and analyzed by the mass spectrometer at a rate of $20 \mathrm{ml} \cdot \mathrm{min}^{-1}$. The resultant gas decay curves were recorded on two, two-channel, twelve inch full scale linear chart recorders (Linear Instruments Corp., Costa Mesa, CA) at a paper speed of $16 \mathrm{in} \cdot \mathrm{min}^{-1}$.

Rebreathe manoeuvers were performed in duplicate following $\mathrm{VO}_{2}$ and CTI measurements at rest and at each target $\mathrm{HR}$, with at least $45 \mathrm{~s}$ separating each rebreathe, to allow for complete elimination of $\mathrm{C}_{2} \mathrm{H}_{2}$ gas. Before each rebreathe, the mass spectrometer was calibrated with a two point calibration using a gas of known concentration $\left(40 \% \mathrm{O}_{2}, 1 \%\right.$ $\mathrm{C}_{2} \mathrm{H}_{2}$, and balance $\mathrm{N}_{2}$ ).

\section{i) Calculation of Cardiac Output}

The $\mathrm{O}_{2}$, and $\mathrm{C}_{2} \mathrm{H}_{2}$, tracings obtained from a rebreathe manoeuvre are shown in Figure 2. Slopes for each gas decay curve were manually drawn by visual inspection. Two points from each curve, corresponding to the same time interval, were selected for the calculation of $\mathrm{a}-\overline{\mathrm{v}} \mathrm{DO}_{2}$. The time intervals and fractional concentrations of gas at each workload were entered into a computer pre-programmed with equations for the calculation a- $\mathrm{vDO}_{2} \mathrm{Q}$ and 


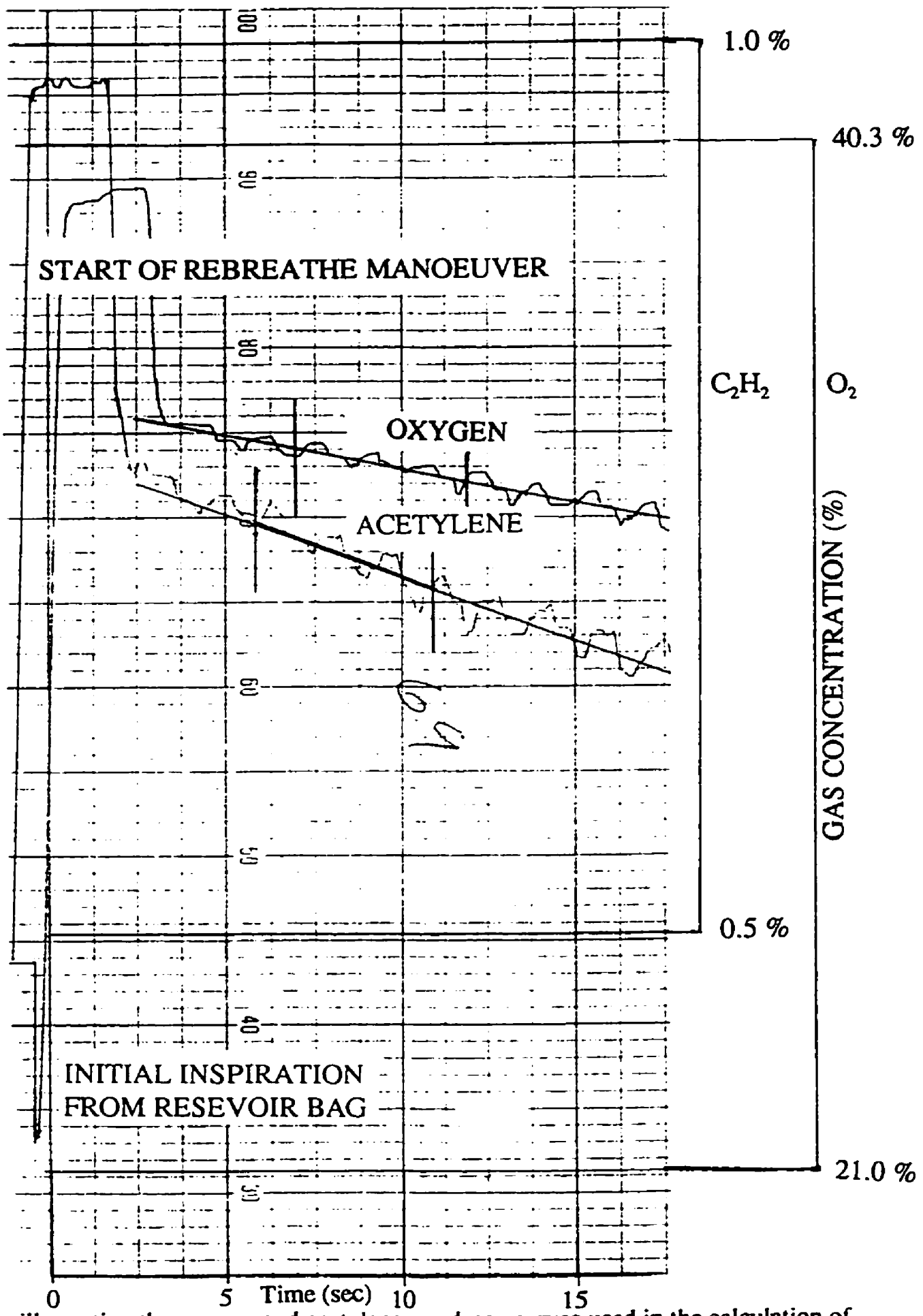

Figure 2. Diagram illustrating the oxygen and acetylene gas decay curves used in the calculation of $\mathrm{a}-\overline{\mathrm{v}} \mathrm{DO}_{2}$. 
SV (see Appendix C). The average of two separate rebreathe manoeuvers performed at rest and at each target $H R$ was used to calculate the final reported $Q$ value. The reduction and calculation of all data from the rebreathe manouvers was performed by the same experimenter to control for the effects of inter-tester variability. In addition, a second experimenter periodically validated the results. The coefficient of variation expressed as the standard deviation divided by the mean difference between duplicate determinations of $Q$ was $4.6 \%$ at rest, $2.7 \%$ at $110 \mathrm{bpm}, 3.8 \%$ at $130 \mathrm{bpm}, 2.4 \%$ at $150 \mathrm{bpm}, 1.7 \%$ at $170 \mathrm{bpm}$ and 1.1 $\%$ at HRmax in all four groups.

\section{Determination of Diastolic Filling Rate and Left Ventricular Ejection Rate}

The DFR (ml-s $\mathrm{s}^{-1}$ was calculated by dividing SV (ml) by DT (s) at rest and at each target HR. The LVER $\left(\mathrm{ml} \cdot \mathrm{s}^{-1}\right)$ was calculated by dividing SV $(\mathrm{ml})$ by LVET $(\mathrm{s})$ at rest and at each targc: $H R$.

\section{Haematology Measurements}

Upon arrival to the laboratory, on each of the two testing days, $5 \mathrm{ml}$ of blood was withdrawn for the determination of $[\mathrm{Hb}]$ and $\mathrm{Hct}$. A rubber tourniquet was placed around the participant's upper arm. A 21 gauge butterfly catheter with micro-plastic tubing attached, (Venisystems ${ }^{\mathrm{TM}}$, Ireland Ltd., Sligo, Rep. of Ireland) was inserted into the brachial vein and the tourniquet was removed. A heparinized (1000 U.S.P. unit $\cdot \mathrm{ml}^{-1}$, Organon Teknika Inc., Toronto, CAN) disposable plastic syringe (Becton-Dickinson and Co., Rutherford, NJ) was used for the collection of the blood sample. 


\section{i) Measurement of Haemoglobin}

Haemoglobin was measured in quadruplicate using the cyanmethaemoglobin method (Sigma Technical Manual, No. 525, Sigma Chemical Co., St. Louis, MO). Five ml of Drabkin's Reagent was pipetted into each of 4 disposable glass test tubes (Fisher Scientific, Pittsburgh, PENN) with a Gilson autopipettor (Mandel Scientific Co., Rockwood, ON). Whole blood was placed into each test tube using $20 \mu \mathrm{l}$ disposable micropipetes (Fischer Scientific, Nepean, ON). The absorbance values were read at a wavelength of $540 \mathrm{~nm}$ on a spectrophotometer (Spectronic 70, Bausch \& Lomb, Rochester NY). A [Hb] standard of 18 $\mathrm{g} \cdot \mathrm{dl}^{-1}$ was used for the standard absorbence reading. The mean value of four determinations was reported as the final $[\mathrm{Hb}]$ value. The coefficient of variation for the determination of $[\mathrm{Hb}]$, expressed as the standard deviation divided by the mean difference between quadruplicate measurements, wâs $2.2 \%$ in group $20,1.6 \%$ in the group $40,1.0 \%$ in group 50 , and $0.8 \%$ in group 60 .

ii) Measurement of Haematocrit

Haematocrit was also measured in quadruplicate using disposable pre-heparinized micro-haematocrit capillary tubes (Fischer Scientific, Pittsburgh, PA). The capillary tubes were sealed (Critoseal, Oxford Labware, St. Louis, MO) and placed inside a centrifuge (International Equipment Co., Needham, MASS). After spinning for $10 \mathrm{~min}$ at 7,000 rpm, the Hct was determined on a micro- haematocrit reader (Model CR, International Equipment Co., Needham, MASS). The mean value of four determinations was reported as the final Hct value. No correction was made for the trapped plasma to allow for comparisons to the 
literature. The coefficient of variation for the determination of Hct, expressed as the standard deviation divided by the mean difference between quadruplicate measurements, was $1.1 \%$ in group $20,0.8 \%$ in the group $40,0.8 \%$ in group 50 , and $1.3 \%$ in group 60 .

\section{Determination of Blood Volume}

Blood Volume was measured using Evan's blue dye (New World Trading Corp., Longwood, CA) in a standard laboratory dilution technique according to the method of Chien et al (26). Participants were instructed to report to the laboratory in the morning, in a fasted state (at least $12 \mathrm{~h}$ ) and to have refrained from heavy training and the consumption of alcohol and caffeine $24 \mathrm{~h}$ prior to the BV measurement. Upon arrival to the laboratory, participants rested semi-reclined for a period of $20 \mathrm{~min}$. During this time, approximately $3 \mathrm{ml}$ of dye ( 5 $\mathrm{mg} \cdot \mathrm{ml}^{-1}$ ) was weighed (Mettler P162, Zurich, SWITZ) to determine the exact volume and was pre-measured into a $5 \mathrm{ml}$ pre-heparinized disposable plastic syringe. A tourniquet was again placed around the participant's upper arm. A 21 gauge butterfly catheter, with a three-way stopcock attached (Baxter Healthcare Corp., Pharmaseal Division, Valencia, CA) was inserted into the brachial vein and the tourniquet was removed. Prior to the dye injection, a $10 \mathrm{ml}$ sample of blood was withdrawn as a "Dye-Free" reference sample. In addition, a $5 \mathrm{ml}$ sample was removed for the determination of $|\mathrm{Hb}|$ and $\mathrm{Hct}$ (refer to previous section). The dye was then slowly injected through the catheter, into the brachial vein. To ensure complete infusion of all traces of dye, the syringe was flushed twice with $10 \mathrm{ml}$ of heparinized saline solution. After a period of $10 \mathrm{~min}$, marked from the beginning of the injection of the dye, a $10 \mathrm{ml}$ blood sample containing "Dyed Plasma" was removed from the opposite arm. The 
blood samples were placed in a centrifuge and spun at $7,000 \mathrm{rpm}$ for $20 \mathrm{~min}$ or until the plasma and red blood cells separated. The absorbence values for the "Dye-Free" and "Dyed Plasma" samples were read at a wavelength of $610 \mathrm{~nm}$ on a spectrophotometer (Spectronic 70, Bausch \& Lomb, Rochester NY). Total PV and BV were calculated according to the formula listed in Appendix D. Blood volumes were reported in both absolute terms (ml) and relative to body mass $\left(\mathrm{ml} \cdot \mathrm{kg}^{-1}\right)$. Changes in the control BV and PV were calculated from the measurements of $[\mathrm{Hb}]$ and Hct using the principle of mass balance (19). Correction was made for the trapped plasma and venous-to-whole body ratio. The coefficient of variation for the determination of $\mathrm{BV}$, expressed as the standard deviation divided by the mean difference between quadruplicate measurements was $3.8 \%$.

\section{Anthropometric Measurements}

Body mass was measured to the nearest $0.01 \mathrm{~kg}$ with participants wearing shorts and t-shirts and with the shoes off using a calibrated balance scale (Gold Brand, Model LPS-100, Taiwan, R.O.C.). Skinfold measurements were performed to determine LBM (kg). Seven skinfold sites (chest, axilla, triceps, subscapular, abdominal, supra iliac and front thigh) were measured to the nearest $0.1 \mathrm{~mm}$ on the right side of the body using a calibrated Harpenden skinfold caliper (John Bull, British Indicators Inc., ENG). The sum of the 7 sites was entered into a computer pre-programmed with an equation derived by Jackson and Pollock used for the calculation of body density of females (134) and equations by Brozek et al. (16) used for the calculation of percentage body fat (see Appendix E). Lean body mass was calculated based on the participant's body mass and percentage body fat. The same experimenter 
performed all skinfold measurements to control for inter-tester variability. The participant's height was measured to the nearest $\mathrm{mm}$ with the shoes off.

\section{Determination of Lung Volume}

Forced vital capacity was determined by multiple measurements on an Auto Spirometer (Pocket Spirometer, Micro Medical Ltd., Rochester, Kent, ENG). Participants were instructed to inhale maximally, then to plug their nose, insert the mouthpiece and exhale as completely and as rapidly as possible. The autospirometer converts air flow into a digital readout of FVC so that comparison can be made to predicted FVC values in the normal range for a person's age, height and sex ( $280 \%$ of normal).

\section{Statistical Analyses}

Descriptive and inferential statistics of all data were conducted using STATISTICA ${ }^{\mathrm{TM}}$ (STATSOFT ${ }^{c}$, Inc., Tusla, OK). Statistical significance was set at $\leq 0.05$. All measurements were reported as means and their standard errors. The dependent variables: $\mathrm{VO}_{2}, \mathrm{a}-\mathrm{vDO}_{2}$, Q SV, SBP, DBP, MAP and TPR were analyzed using a mixed-model, two-way analysis of variance (ANOVA) with four levels of group (between) and six levels of HR (within). Tukey post-hoc tests (for unequal $\mathrm{n}$ ) were used to further analyze significant differences in measurements at rest and at each target HR, as well as differences between groups. As previously reviewed, since LVET and PEP are inversely and linearly related to HR (292, 293), and a non-linear relationship exists between DT and HR, small variations in HR may lead to significant changes in CTI. Thus, to interpret changes and/or differences in CTI between individuals at rest, correction must be made for variations in HR (292). Since the 
correction factors for LVET and PEP were only validated for resting HR, and since no such factor has been created for DT, individuals must be examined at matched HR during exercise. Since the last matched HR of all four groups was $150 \mathrm{bpm}$, participants could not be statistically compared beyond a HR of $150 \mathrm{bpm}$. Thus, the CTI (LVET, PEP and DT) and the DFR and LVER (which were calculated from CTI measurements) were analyzed using a mixed-model, two-way ANOVA with four levels of group (between) and four levels of HR (within). Tukey post-hoc tests (for unequal $\mathrm{n}$ ) were used to further analyze significant differences in measurements at rest and at each target HR, as well as differences between groups. One-way repeated measures ANOVA's were performed on all variables at max exercise and on the CTI, DFR and LVER at $150 \mathrm{bpm}$. Regression analyses were conducted to analyze SV slopes for significance and to determine the $\mathrm{Q}-\mathrm{VO}_{2}$ relationships for each group. 


\section{CHAPTER IV}

\section{RESULTS}

This chapter contains a summary of the results and statistical analyses of the data. All values are reported as the mean \pm SEM. The raw data for all measurements are presented in Appendix A. Only those significant group differences which were found using a mixed model, two-way ANOVA will be shown on the figures.

\section{Particinant Characteristics}

A summary of the participants' characteristics are presented in Table 2. As expected, the mean ages of the participants in all four groups differed significantly from one another (Group $20=22.4 \pm 1.4 \mathrm{yr}$, Group $40=42.7 \pm 0.6 \mathrm{yr}$, Group $50=50.8 \pm 0.8 \mathrm{yr}$ and Group $60=60.3 \pm 1.2 \mathrm{yr}$ ). Both groups 50 and 60 had a significantly lower HRmax (167 \pm 4 and $157 \pm 4$ bpm, respectively) than both groups 20 and 40 (192 \pm 1 and $182 \pm 1$ bpm, respectively). However, there were no differences in HRmax between groups 20 and 40 or between groups 50 and 60 . Additionally, there were no group differences in the number of participants who were able to reach their age-predicted $H R \max (5 / 6,6 / 6,5 / 6$ and $4 / 5$, for groups $20,40,50$ and 60 respectively). No group differences were observed for resting HR or for FVC. Group 20 had a significantly larger $\mathrm{VO}_{2} \max \left(63.7 \pm 2.3 \mathrm{ml} \cdot \mathrm{kg}^{-1} \cdot \mathrm{min}^{-1}\right)$ than all other groups (Group $40=51.0 \pm 3.1$, Group $50=47.0 \pm 2.5$ and Group $60=44.2 \pm 1.7$ $\left.\mathrm{ml} \cdot \mathrm{kg}^{-1} \cdot \mathrm{min}^{-1}\right)$. No other significant group differences existed for $\mathrm{VO}_{2} \mathrm{max}\left(\mathrm{ml} \cdot \mathrm{kg}^{-1} \cdot \mathrm{mirt}\right)$. Body mass $(\mathrm{kg})$, relative percentage body fat, LBM $(\mathrm{kg})$ and height $(\mathrm{cm})$ did not differ between groups. In addition, there were no group differences for $[\mathrm{Hb}]\left(\mathrm{g} \cdot 100 \mathrm{ml}^{-1}\right)$, Hct $(\%)$, 
Table 2. Summary of participants' characteristics and cardiac function.

\begin{tabular}{|c|c|c|c|c|c|c|c|c|c|c|c|}
\hline Group & $\begin{array}{l}\text { Age } \\
\text { (yr) }\end{array}$ & $\begin{array}{l}\text { Body } \\
\text { Mass } \\
\text { (kg) }\end{array}$ & $\begin{array}{l}\text { LeM } \\
(\mathrm{kg})\end{array}$ & $\begin{array}{l}\text { Holght } \\
\text { (cm) }\end{array}$ & $\underset{\text { (bpm) }}{\text { MRmax }}$ & $\begin{array}{l}\text { BV } \\
\text { (m) }\end{array}$ & $\begin{array}{c}\text { av } \\
\left(\mathbf{m l} \cdot \mathbf{k} g^{-1}\right)\end{array}$ & (ax) & $\underset{\left(m l \cdot k \sigma^{-1} \cdot m l n^{-1}\right)}{v{ }^{\prime}}$ & $\begin{array}{l}\text { vo } \max \\
\left(1+\min \mid n^{-1}\right)\end{array}$ & $\underset{\left(1-\min n^{-1}\right)}{\max }$ \\
\hline 20 & $22.4 \pm 1.4^{*}$ & $60.3 \pm 19$ & $51.2 \pm 1.9$ & $166.5 \pm 2.4$ & $192 \pm 1^{\prime \prime}$ & $5039 \pm 164$ & $83.6 \pm 1.5$ & $14.4 \pm 0.3$ & $63.7 \pm 2.3^{\circ}$ & $3.85 \pm 0.14^{\circ}$ & $24.1 \pm 0.9^{\circ}$ \\
\hline 40 & $42.7 \pm 0.6 \dagger$ & $62.6 \pm 13$ & $51.4 \pm 1.4$ & $164.7 \pm 2.6$ & $182 \pm 1 \dagger$ & $4812 \pm 276$ & $76.8 \pm 3.5$ & $14.0 \pm 0.3$ & $51.0 \pm 3.1$ & $3.19 \pm 0.19$ & $18.6 \pm 1.1$ \\
\hline 60 & $60.3 \pm 1.2$ & $56.9 \pm 2.8$ & $46.3 \pm 2.3$ & $163.9 \pm 2.4$ & $157 \pm 4$ & $4552 \pm 395$ & $79.7 \pm 4.8$ & $14.4 \pm 0.2$ & $44.2 \pm 1.7$ & $2.51 \pm 0.16$ & $15.9 \pm 0.8$ \\
\hline
\end{tabular}

\begin{tabular}{|c|c|c|c|c|c|c|c|c|c|c|c|}
\hline Group & $\underset{(m l)}{S V \max }$ & $\begin{array}{c}\text { a-vDO } \mathrm{ymax}_{2} \\
\left(\mathrm{ml} \cdot 100 \mathrm{ml}^{-1}\right)\end{array}$ & $\begin{array}{l}\text { LVET150 } \\
\text { (msec) }\end{array}$ & $\begin{array}{r}\text { PEPT50 } \\
\text { (meec) }\end{array}$ & $\begin{array}{l}\text { DT1\$0 } \\
\text { (msec) }\end{array}$ & $\begin{array}{l}\text { DFR150 } \\
\left(m+\cdot 8 e c^{-1}\right)\end{array}$ & $\begin{array}{l}\text { LVER150 } \\
\left.\text { (ml-sec }{ }^{-1}\right)\end{array}$ & $\begin{array}{l}\text { SBPmax } \\
\text { (mmlta) }\end{array}$ & $\begin{array}{l}\text { DBPmax } \\
\text { (mmitg) }\end{array}$ & $\underset{\text { (mmHg) }}{\operatorname{MAPmax}}$ & $\underset{\substack{\text { TPRmitgd } \\
\text { (minat }}}{\min n^{-1}}$ \\
\hline 20 & $125.4 \pm 4.7$ & $16.0 \pm 5.1$ & $204 \pm 5$ & $48 \pm 10$ & $147 \pm 6$ & $733 \pm 39$ & $525 \pm 27$ & $182 \pm 5^{-\cdots}$ & $66 \pm 2$ & $104.5 \pm 2.6$ & $4.37 \pm 0.20^{\circ \bullet}$ \\
\hline 40 & $109.4 \pm 5.6$ & $16.3 \pm 32$ & $213 \pm 9$ & $28 \pm 6$ & $164 \pm 8$ & $627 \pm 20$ & $482 \pm 26$ & $177 \pm 8$ & $68 \pm 2$ & $104.2 \pm 3.7$ & $5.38 \pm 0.30+$ \\
\hline 60 & $104.0 \pm 7.2$ & $15.8 \pm 2.3$ & $217 \pm 6$ & $32 \pm 7$ & $155 \pm 8$ & $667 \pm 30$ & $475 \pm 29$ & $192 \pm 7$ & $82 \pm 5$ & $118.1 \pm 5.0$ & $7.48 \pm 0.43$ \\
\hline
\end{tabular}

Values are means \pm SEM

- Group 20 significantly different than Groups 40, 50, 60 (P<0,05).

-. Group 20 significantly ditterent than Groups 50, 60 (P<0.05).

-.. Group 20 significantly different than Group 60 (P<0.05).

+ Group 40 significantly difterent than Group 60 (P<0.05).

$t$ Group 40 significantly different than Groups $50,60(P<0.05)$

₹ Group 50 significantly different than Group 60 (P<0.05). 
or BV $(\mathrm{ml})$, (ml. $\left.\mathrm{kg}^{-1}\right)$ or $\left(\mathrm{ml} \cdot \mathrm{kg} \mathrm{LBM}^{-1}\right)$. Blood volume in $\mathrm{ml}$ and $\mathrm{ml} \cdot \mathrm{kg}^{-1}$ as a function of age is illustrated in Figure 3.

\section{Effects of Age on Cardiac Function}

The primary purpose of this investigation was to examine the effects of age (group) on the cardiac function of ET females at rest and during incremental exercise to maximum. The only group difference that existed at rest was in $\mathrm{a}-\mathrm{vDO}_{2}$, which was significantly higher in groups 40 and 60 compared to group $20(10.2 \pm 0.46$ and $9.57 \pm 0.26$ vs $7.78 \pm 0.31 \mathrm{ml}$ $\mathrm{O}_{2} \cdot 100 \mathrm{ml}^{-1}$ ). A summary of the participants' data during maximal exercise are reported in Table 2. Maximal oxygen uptake $\left(1 \cdot \mathrm{min}^{-1}\right)$ was significantly lower in groups 40,50 and 60 compared to group 20. The smaller $\mathrm{VO}_{2} \max \left(1 \cdot \mathrm{min}^{-1}\right)$ in groups 40,50 and 60 was due to a significantly lower $Q \max \left(1 \cdot \mathrm{min}^{-1}\right)$, in these three groups compared to group 20, whereas no significant group difference was observed for $\mathrm{a}-\overline{\mathrm{v}} \mathrm{DO}_{2} \max \left(\mathrm{ml} \mathrm{O}_{2} \cdot 100 \mathrm{ml}^{-1}\right)$. The smaller Qmax $\left(1 \cdot \mathrm{min}^{-1}\right)$ in groups 40,50 and 60 was due to a significantly lower HRmax (bpm) compared to group 20, whereas no significant group difference was observed for SVmax (ml). The TPRmax $\left(\mathrm{mmHg} \cdot \mathrm{f}^{-1} \cdot \mathrm{min}^{-1}\right)$ was significantly higher in groups 50 and 60 compared to group 20, and in group 60 compared to group 40 . The higher TPRmax $\left(\mathrm{mmHg} \cdot \mathrm{l}^{-1} \cdot \mathrm{min}^{-1}\right)$ in groups 50 and 60 was due to a higher MAPmax and a lower $Q \max \left(1 \cdot \mathrm{min}^{-1}\right)$ in these two groups compared to group 20.

\section{exygen Uptake}

In Figure $4, \mathrm{VO}_{2}\left(1 \cdot \mathrm{min}^{-1}\right)$ is illustrated as a function of $\mathrm{HR}$ and group and in Figure $5, \mathrm{VO}_{2}\left(\mathrm{ml} \cdot \mathrm{kg}^{-1} \cdot \mathrm{min}^{-1}\right)$ is shown as a function of $\mathrm{HR}$ and group. The analysis of $\mathrm{VO}_{2}$ values 


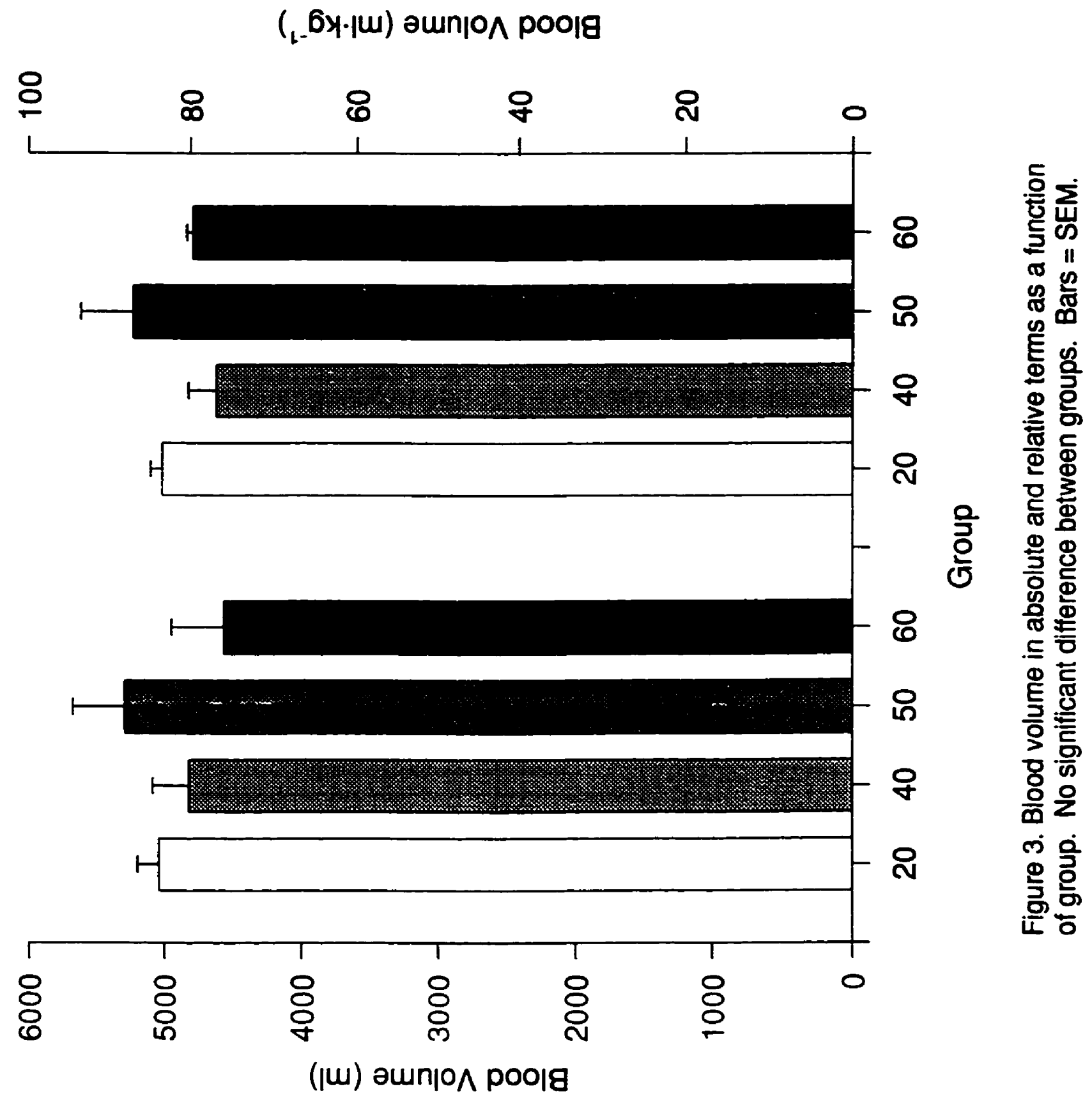




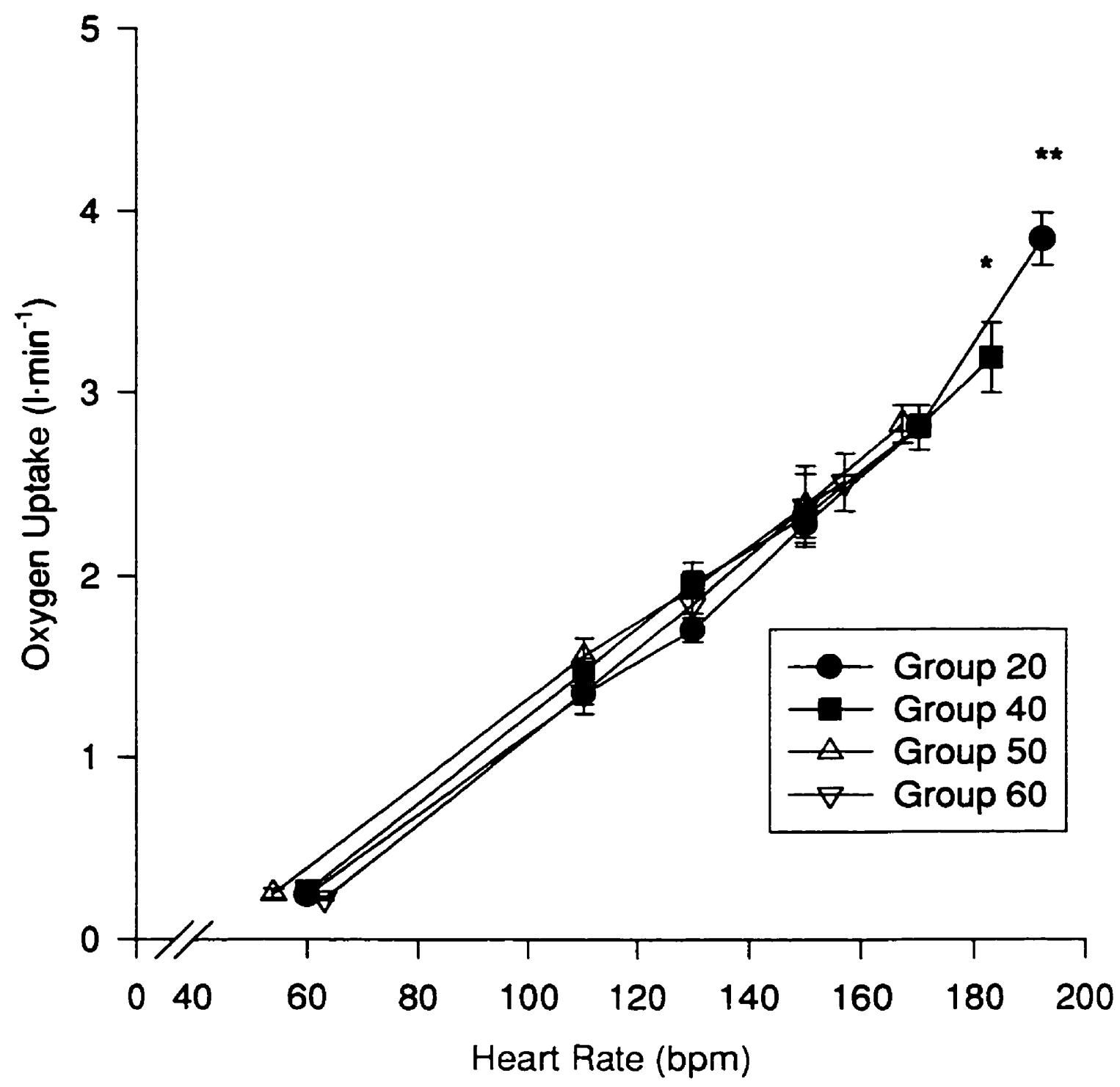

Figure 4. Oxygen uptake as a function of $\mathrm{HR}$ and group.

- Group $40>$ Groups 50 and 60 at HRmax $(p<0.05)$.

** Group $20>$ than all other groups at HRmax $(p<0.05)$.

Bars $=$ SEM. 


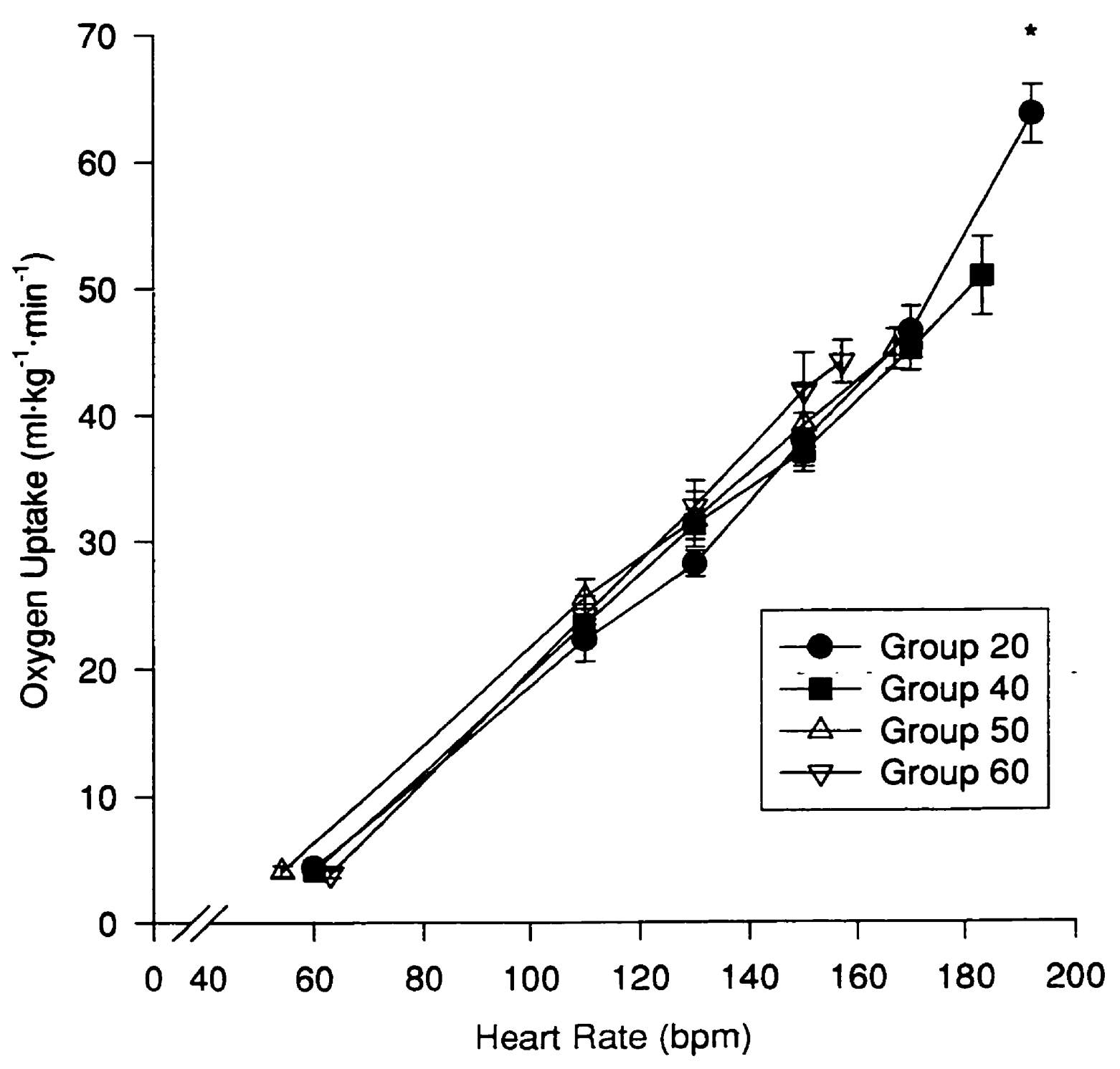

Figure 5. Oxygen uptake as a function of HR and group.

- Group $20>$ than all other groups at HRmax $(p<0.05)$.

Bars $=$ SEM. 
revealed a significant interaction effect between $\mathrm{HR}$ and group in both $1 \cdot \mathrm{min}^{-1}$ and $\mathrm{ml} / \mathrm{kg}^{-1}$. $\min ^{-1}(F(15,95)=7.91, p<0.0001$ and $F(15,95)=7.51, p<0.0001$, respectively). Tukey post-hoc analysis of the $\mathrm{HR}$ and group interaction revealed that $\mathrm{VO}_{2}$ was significantly different at all HR, in all groups, such that it increased progressively with each increment in work rate, up to maximum. Tukey post-hoc analysis of the interaction effect also revealed that $\mathrm{VO}_{2} \max$ $\left(1 \cdot \mathrm{min}^{-1}\right)$ was significantly lower in groups $40(17 \%), 50(26 \%)$ and $60(35 \%)$ compared to group 20. Additionally, the $\mathrm{VO}_{2} \max \left(1 \cdot \mathrm{min}^{-1}\right)$ was significantly lower in groups $50(10 \%)$ and $60(21 \%)$ compared to group 40 . No group differences were observed at any of the other target HR. Tukey post-hoc analysis of the interaction effect also revealed that $\mathrm{VO}_{2}$ max $\left(\mathrm{ml} \cdot \mathrm{kg}^{-1} \cdot \mathrm{min}^{-1}\right)$ was significantly lower in groups $40(20 \%), 50(26 \%)$ and $60(31 \%)$ compared to group 20 and in group $60(26 \%)$ compared to group 40 (Table 2). Similarly, the independent analysis of $\mathrm{VO}_{2}$ values at $\max (F(3,19)=12.13, \mathrm{p}<0.001$ and $\mathrm{F}(3,19)=11.63$, $\mathrm{p}<0.001$ ) revealed that $\mathrm{VO}_{2} \max$ in $1 \cdot \mathrm{min}^{-1}$ and in $\mathrm{ml} \cdot \mathrm{kg}^{-1} \cdot \mathrm{min}^{-1}$ was significantly lower in groups 40,50 and 60 compared to group 20 (Table 2).

\section{Cardiac Output}

In Figure $6, Q$ is illustrated as a function of $H R$ and group and in Figure $7, Q$ is depicted as a function of oxygen uptake and group. Analysis of $Q\left(1 \cdot \mathrm{min}^{-1}\right)$ revealed that a significant interaction effect existed between HR and group $(F(15,95)=7.05, p<0.0001)$. Tukey post-hoc analysis of this interaction effect showed that $Q$ was significantly different at all $H R$ in all groups, such that it increased progressively with each successive HR up to maximum. Tukey post-hoc analysis of the interaction effect also revealed that $\mathrm{Qmax}$ was 


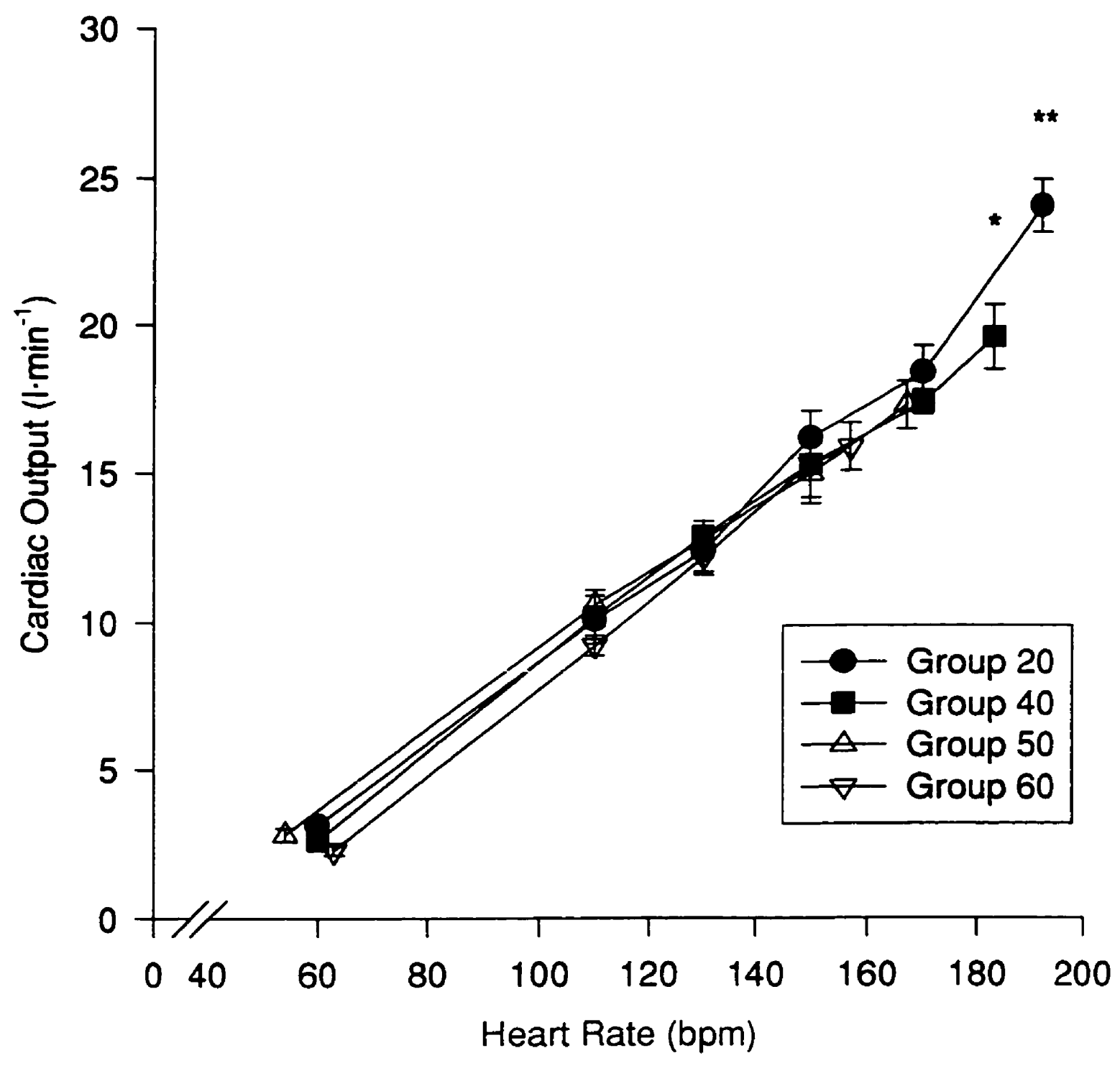

Figure 6. Cardiac output as a function of HR and group.

* Group $20>$ than all other groups at HRmax $(p<0.05)$.

- Group $40>$ Group 60 at HRmax $(p<0.05)$.

Bars $=$ SEM. 


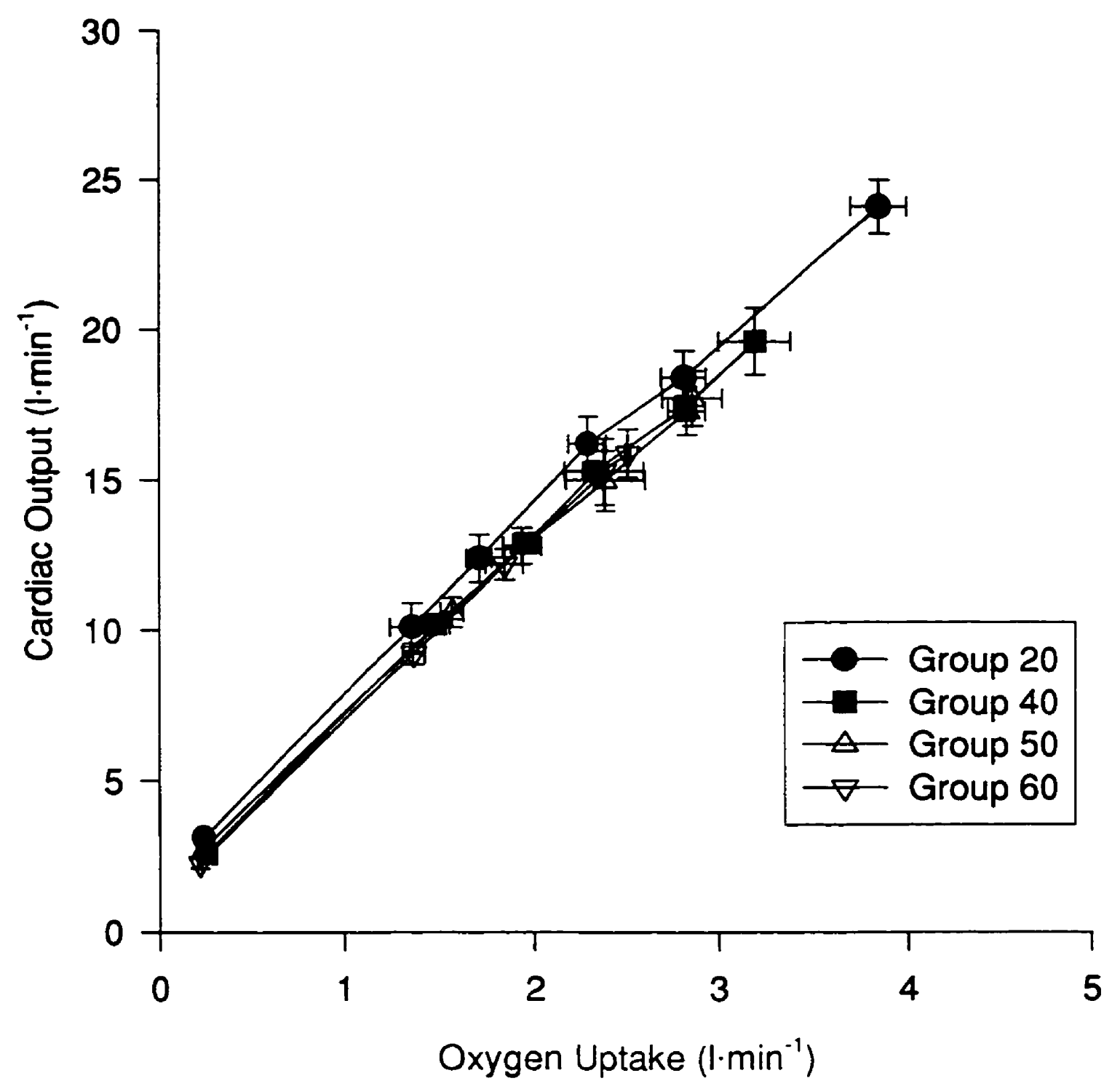

Figure 7. Cardiac output as a function of oxygen uptake and group. Bars $=$ SEM. 
significantly lower in groups $40(19 \%), 50(26 \%)$ and $60(34 \%)$ compared to group 20 . In addition, Qmax was significantly lower in group 60 (19\%) compared to group 40. No other group differences were observed for $Q$ at any of the other target HR. The independent analysis of $\mathrm{Q}$ values at $\max (\mathrm{F}(3,19)=12.52, \mathrm{p}<0.0001)$ revealed that $\mathrm{Qmax}\left(1 \cdot \mathrm{min}^{-1}\right)$ was significantly lower in groups 40,50 and 60 compared to group 20 (Table 2).

The $\mathrm{Q}-\mathrm{VO}_{2}$ relationship was calculated from 3 data points for each participant (58 \pm $0.8,72 \pm 0.2$ and $100 \%$ of $\left.\mathrm{VO}_{2} \max \right)$. Linear regressions were: $\mathrm{Q}\left(1 \cdot \mathrm{min}^{-1}\right)=5.12 \times \mathrm{VQ}$ $\left(1 \cdot \mathrm{min}^{-1}\right)+4.28$ (standard error of the estimate $\left.(\mathrm{SEE})=1.6251\right)$, with $85 \%$ of the variance accounted for in group 20; $\mathrm{Q}\left(1 \cdot \mathrm{min}^{-1}\right)=5.40 \times \mathrm{VO}_{2}\left(1 \cdot \mathrm{min}^{-1}\right)+2.33(\mathrm{SEE}=0.8101)$, with $98 \%$ of the variance accounted for in group $40 ; \mathrm{Q}\left(1 \cdot \mathrm{min}^{-1}\right)=5.30 \times \mathrm{VO}_{2}\left(1 \cdot \mathrm{min}^{-1}\right)+2.46$ $(\mathrm{SEE}=0.6742)$, with $95 \%$ of the variance accounted for in group 50 and $\mathrm{Q}\left(1 \cdot \mathrm{min}^{-1}\right)=5.61$ $\times \mathrm{VO}_{2}\left(1 \cdot \mathrm{min}^{-1}\right)+1.78(\mathrm{SEE}=0.4780)$, with $98 \%$ of the variance accounted for in group 60.

\section{Stroke Volume}

In Figure 8, SV is illustrated as a function of $\mathrm{HR}$ and group and in Figure 9, SV is depicted as a function of oxygen uptake and group. The analysis of SV (ml) values from all conditions revealed a significant main effect for $H R(F(5,95)=198.5, p<0.0001)$. Tukey posthoc analysis of the mean SV pooled across groups revealed significant progressive increases in SV from rest to $110 \mathrm{bpm}, 110 \mathrm{bpm}$ to $150 \mathrm{bpm}, 130 \mathrm{bpm}$ to $\max , 150 \mathrm{bpm}$ to max, and from $170 \mathrm{bpm}$ to max. No group differences were observed for SV, such that age had no significant effect on SV. Further, the independent analysis of SV values at max revealed no significant group differences (Table 2). 


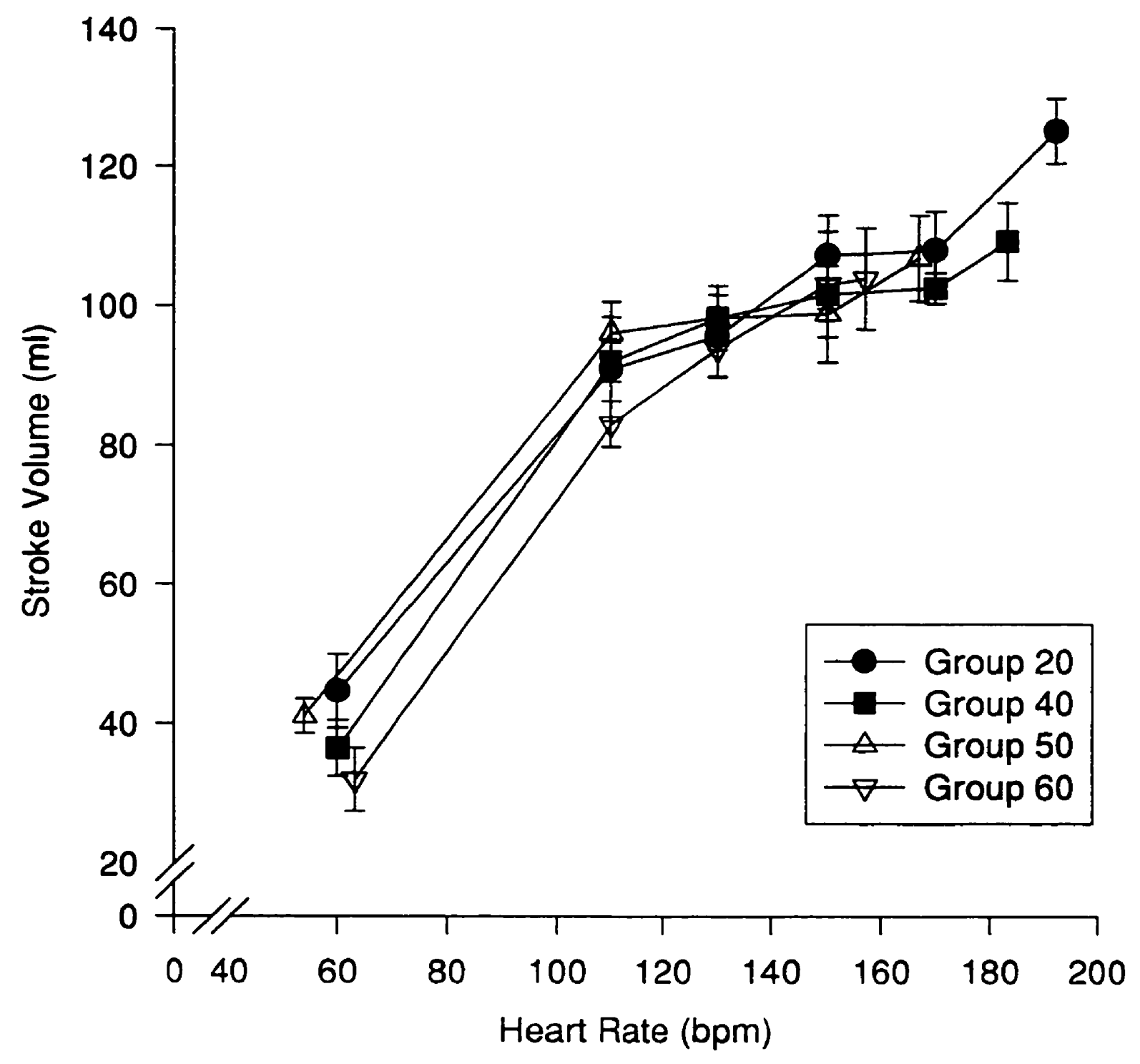

Figure 8. Stroke volume as a function of HR and group. No significant differences between groups. Bars=SEM. 


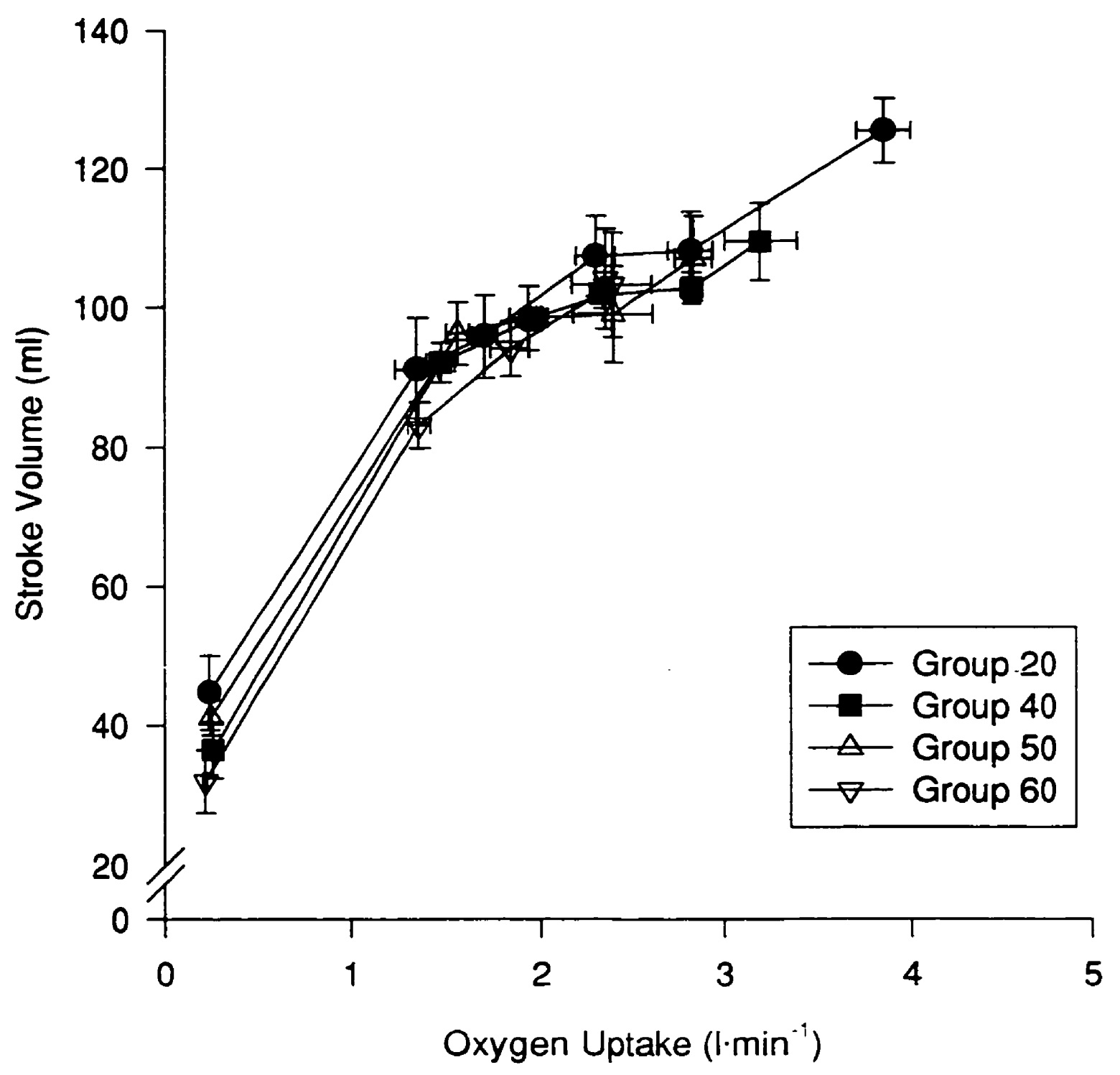

Figure 9. Stroke volume as a function of oxygen uptake and group. No significant differences between groups. Bars $=$ SEM. 
Regression analyses were performed on the exercise SV of each group, using data points from all of the target HR during exercise, for each group. Group 20 had a slope $=$ 0.57 , with $74 \%$ of the variance explained, group 40 had a slope $=0.55$, with $87 \%$ of the variance explained, group 50 had a slope $=0.57$, with $70 \%$ of the variance accounted for and group 60 had a slope $=0.77$, with $81 \%$ of the variance accounted for.

\section{Arterial-Mixed Venous Oxygen Difference}

In Figure 10, a- $\overline{\mathrm{vDO}}{ }_{2}$ is illustrated as a function of $\mathrm{HR}$ and group and in Figure 11 , $\mathrm{a}-\mathrm{iDO}_{2}$ is depicted as a function of oxygen uptake and group. Analysis of a- $\overline{\mathrm{vDO}} 2\left(\mathrm{ml} \mathrm{O}_{2} \cdot 100\right.$ $\left.\mathrm{ml}^{-1}\right)$ revealed a significant interaction effect between $\mathrm{HR}$ and group $(\mathrm{F}(15,95)=2.12, \mathrm{p}<0.05)$. Tukey post-hoc analysis conducted on the interaction effect showed that all groups showed a significant increases in $\mathrm{a}-\overline{\mathrm{v}} \mathrm{DO}_{2}$ from rest to $110 \mathrm{bpm}$. Groups 20,40 and 50 , showed a significant increase in $\mathrm{a}-\overline{\mathrm{vDO}} \mathrm{DO}_{2}$ from $110 \mathrm{bpm}$ to $170 \mathrm{bpm}$. Group 20 also showed a progressive increase in $\mathrm{a}-\mathrm{DDO}_{2}$ from $130 \mathrm{bpm}$ to $\max$ and from $150 \mathrm{bpm}$ to max. Tukey posthoc analysis of the interaction effect also revealed that $\mathrm{a}-\overline{\mathrm{iDO}} \mathrm{O}_{2}$ at rest was significantly greater in group 40 compared to group $20\left(10.2 \pm 4.6\right.$ vs $\left.7.78 \pm 0.31 \mathrm{ml} \mathrm{O}_{2} \cdot 100 \mathrm{ml}^{-1}\right)$. In addition, at $150 \mathrm{bpm} \mathrm{a-}-\mathrm{vDO}_{2}$ was significantly greater in group 50 compared to group $20(15.9 \pm 2.4$ vs $14.2 \pm 5.6 \mathrm{ml} \mathrm{O}_{2} \cdot 100 \mathrm{ml}^{-1}$ ). No other group differences were observed for $\mathrm{a}-\mathrm{v} \mathrm{vO}_{2}$ at any of the other target HR.

\section{Cardiac Time Interyals}

As previously reviewed, since CTI are inversely related to $\operatorname{HR}(292,293)$, small variations in HR may lead to significant changes in CTI. Thus, to interpret changes and/or 


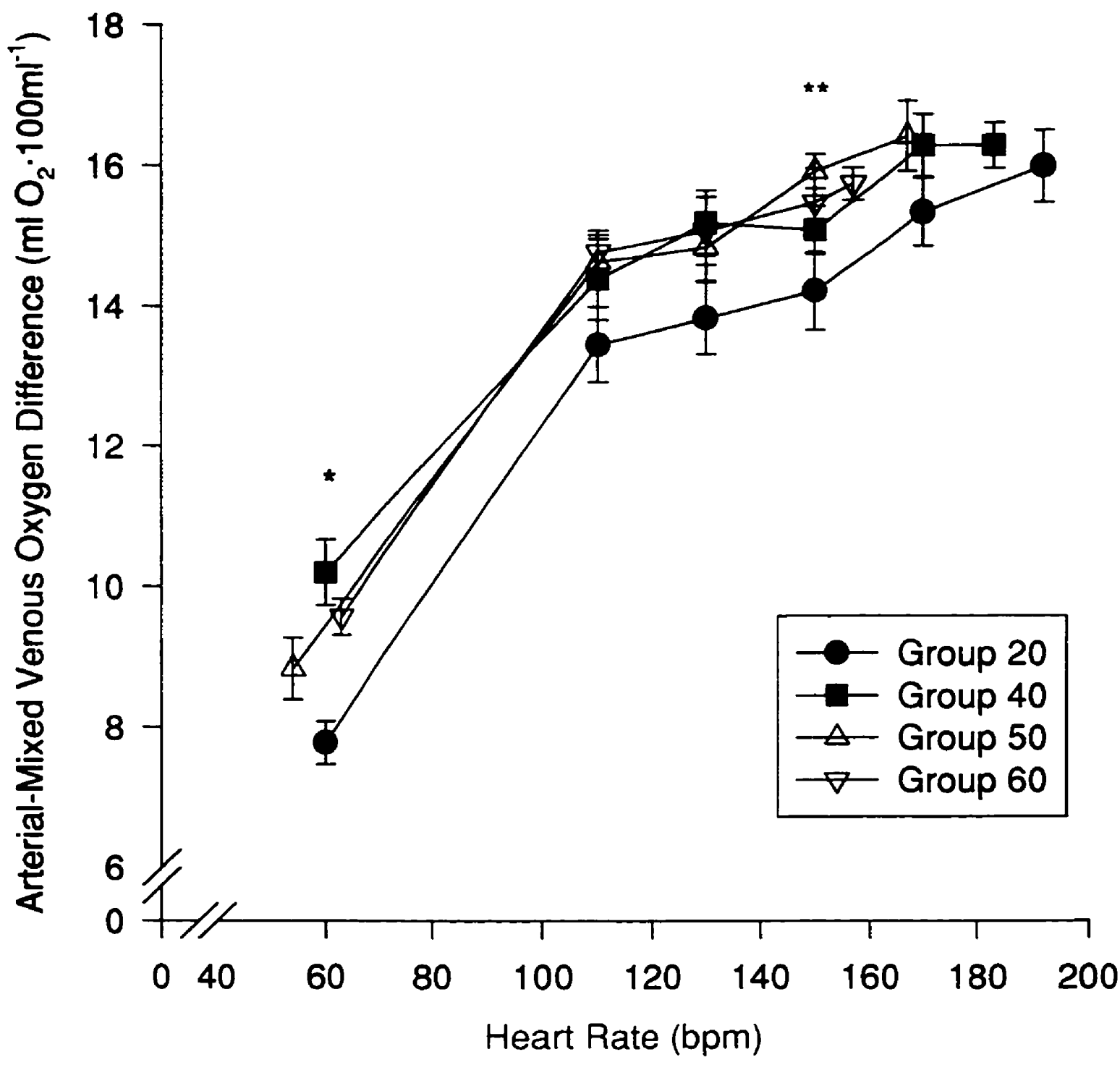

Figure 10. Arterial-mixed venous oxygen difference as a function of HR and group. - Group $40>$ Group 20 at rest $(p<0.05)$. ** Group $60>$ Group 20 at 150 bpm $(p<0.05)$. Bars = SEM. 


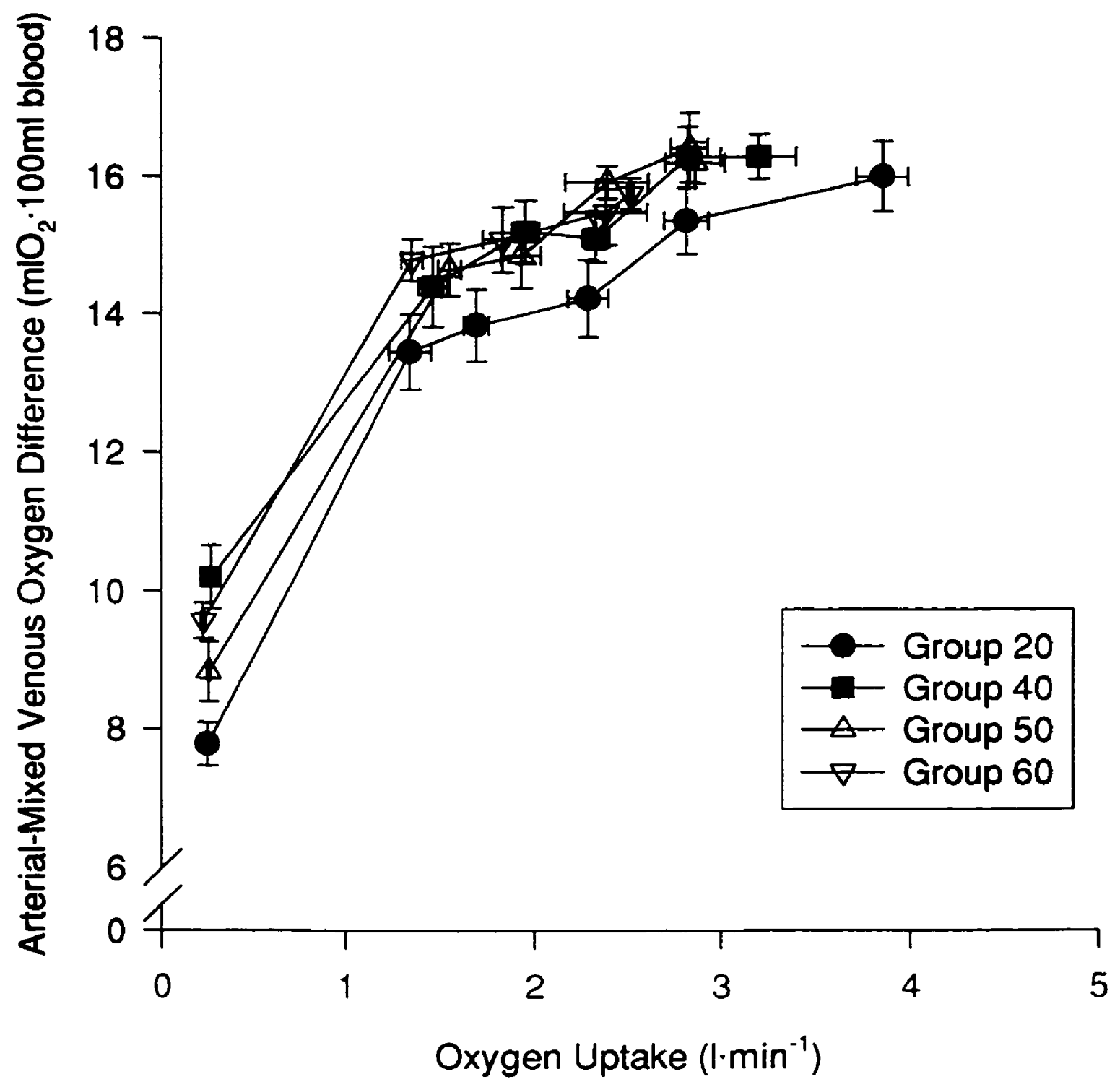

Figure 11. Arterial-mixed venous oxygen difference as a function of oxygen uptake and group. Bars = SEM. 
differences in CTI between individuals, they must be examined at matched HR. Since HR could not be standardized at rest, resting values of LVET and PEP were adjusted for the effects of differences in HR using standard equations (292). However, the correction factors for LVET and PEP were only validated for resting HR, and no such factor has been created for DT. Therefore, since the last matched HR of all four groups was $150 \mathrm{bpm}$, CTI could not be statistically compared between groups beyond a HR of $150 \mathrm{bpm}$.

In Figure 12, LVET is illustrated as a function of HR and group. Analysis of LVET (msec) revealed that as expected there was a significant main effect for $\mathrm{HR}(F(3,57)=1491.8$, $\mathrm{p}<0.0001$ ). Tukey post-hoc analysis of the mean LVET pooled across groups revealed a significant progressive decline with each successive increase in HR up to $150 \mathrm{bpm}$. No group differences were observed for LVET, such that age had no significant effect on LVET. Further, the independent analysis of LVET values at $150 \mathrm{bpm}$ revealed no significant group differences (Table 2).

In Figure 13, PEP is illustrated as a function of HR and group. Analysis of PEP (msec) revealed that as expected there was a significant main effect for $\mathrm{HR}(F(3,57)=378.2$, $p<0.0001$ ). Tukey post-hoc analysis on this main effect showed that incremental exercise resulted in a significant progressive decline in the duration of PEP from rest to $110 \mathrm{bpm}$ and $110 \mathrm{bpm}$ to $130 \mathrm{bpm}$. No group differences were observed for PEP, such that age had no significant effect on PEP. Further, the independent analysis of PEP values at $150 \mathrm{bpm}$ revealed no significant group differences (Table 2).

In Figure 14, DT is illustrated as a function of HR and group. Analysis of DT (msec) 


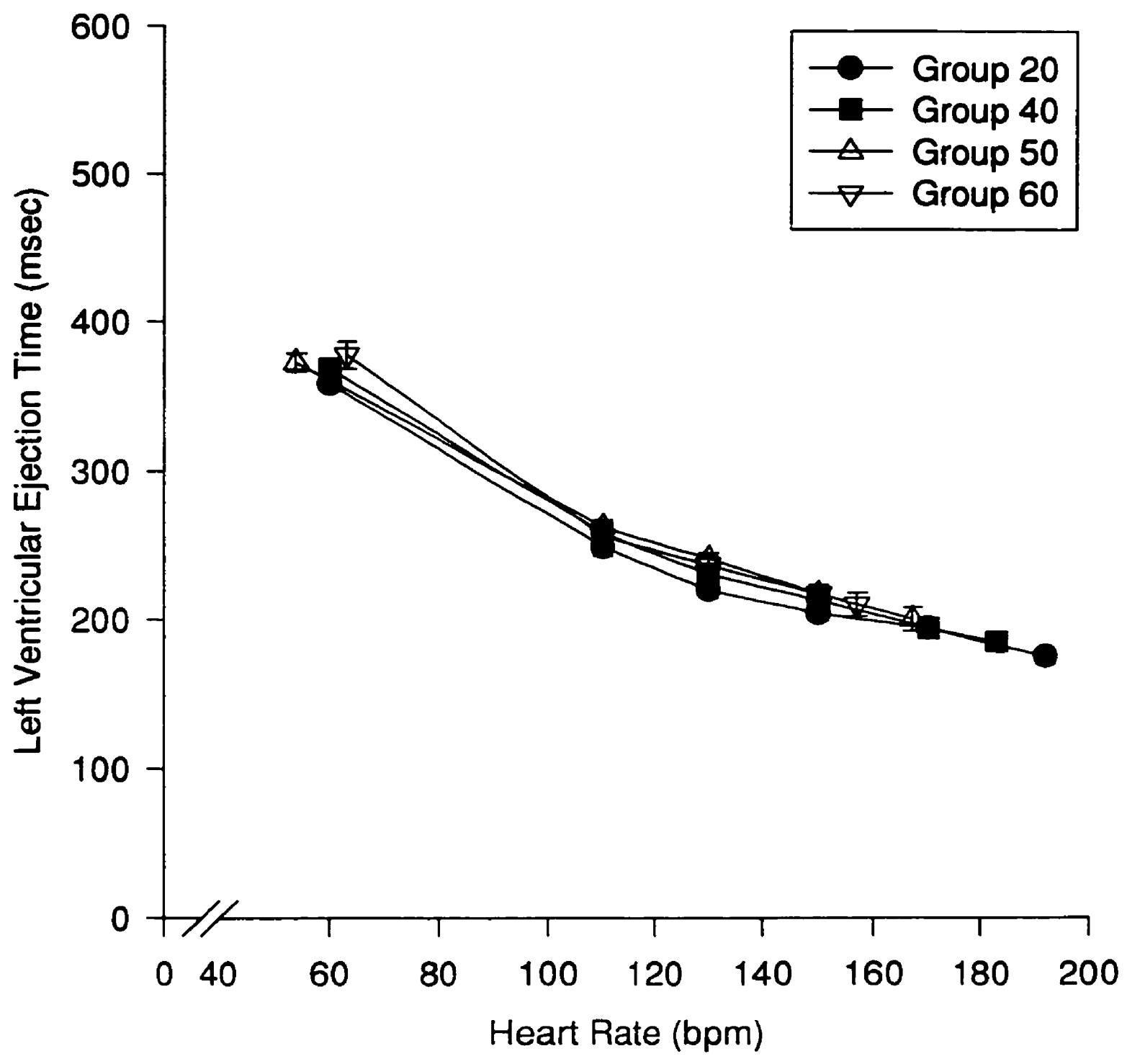

Figure 12. Left ventricular ejection time as a function of $H R$ and group. No significant differences between groups. Bars $=$ SEM. 


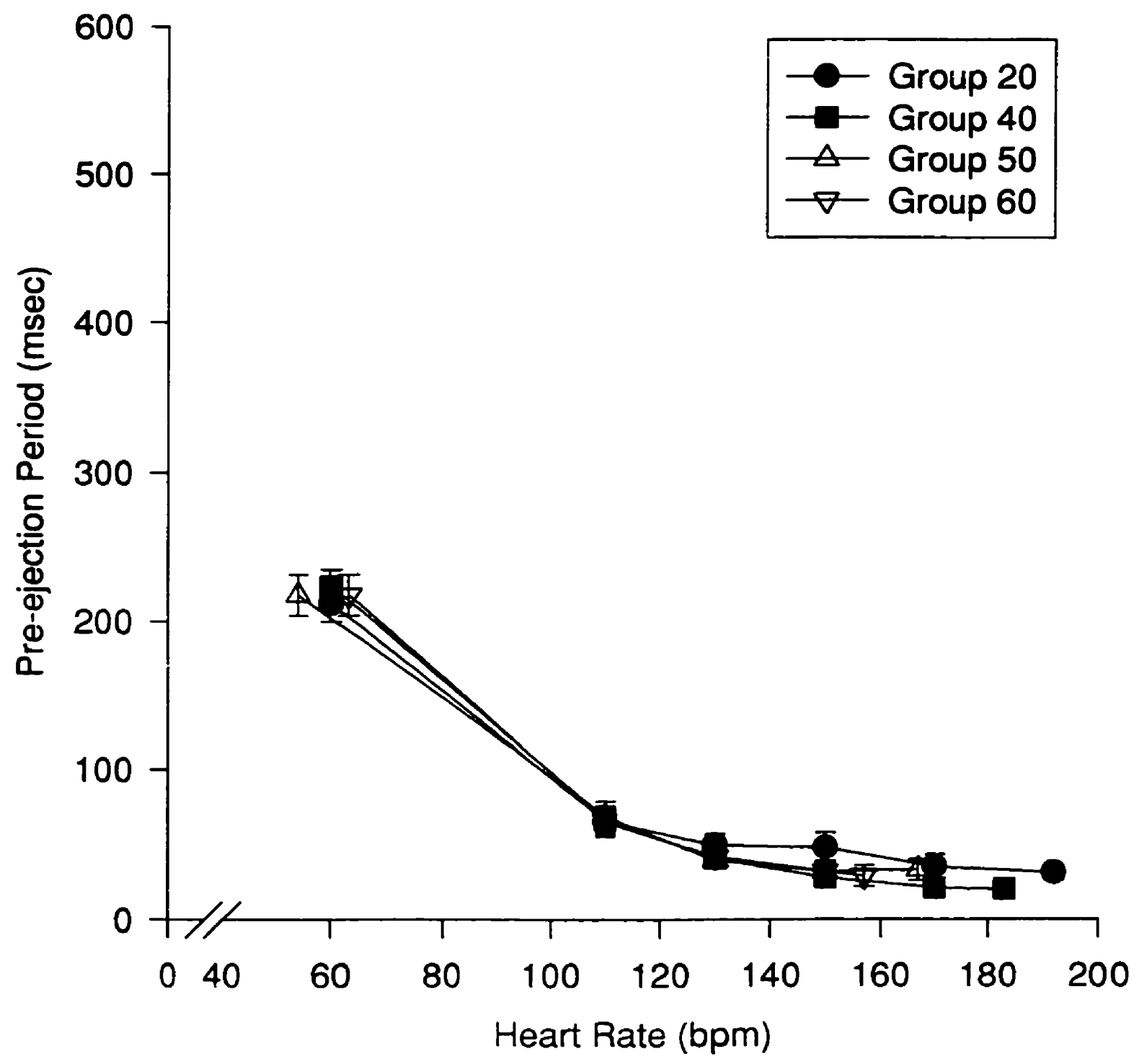

Figure 13. Pre-ejection period as a function of $\mathrm{HR}$ and group. No significant differences between groups. Bars $=$ SEM. 


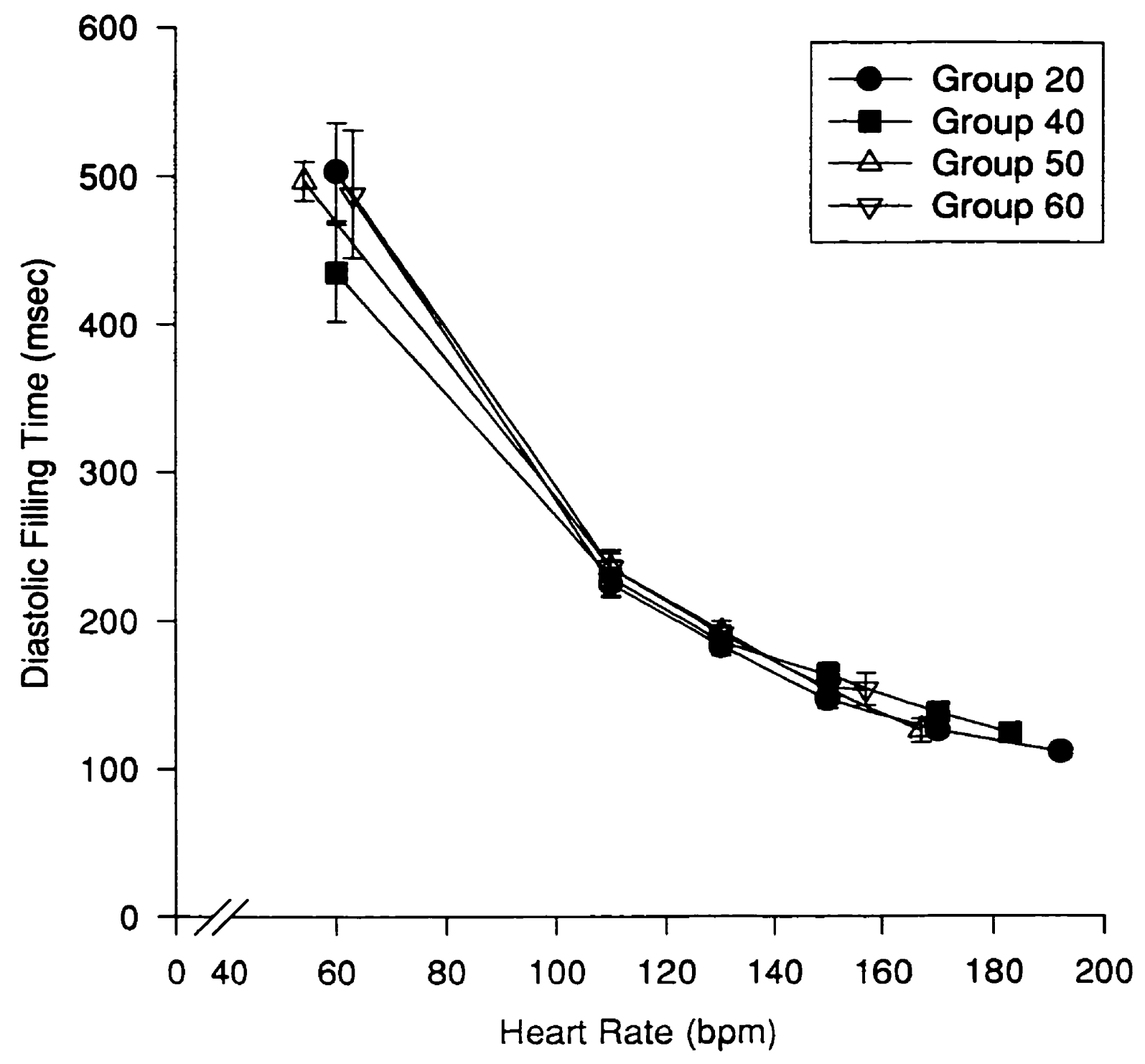

Figure 14. Diastolic time as a function of HR and group. No significant differences between groups. Bars = SEM. 
revealed a significant main effect for $H R(F(3,57)=283.6, p<0.0001)$. Tukey post-hoc analysis of the mean DT pooled across groups revealed a significant progressive decline in the duration of DT with each successive increase in HR up to $150 \mathrm{bpm}$. No group differences were observed for DT, such that age had no significant effect on DT. Further, the independent analysis of DT values at $150 \mathrm{bpm}$ revealed no significant group differences (Table 2).

At matched HR during exercise (excluding $110 \mathrm{bpm}$ ), the duration of LVET was greater than the duration of DT in all four groups. For instance at $150 \mathrm{bpm}$ : in group 20; LVET required $51 \%$ of the cardiac cycle, while DT occupied only $37 \%$, in group 40; LVET required 52\% of the cardiac cycle, while DT occupied only $40 \%$, in group 50; LVET required $54 \%$ of the cardiac cycle, while DT occupied only $38 \%$, and in group 60 ; LVET required $54 \%$ of the cardiac cycle, while DT occupied only $38 \%$ (Table 2 ).

Further, as the cardiac cycles shorten with increasing HR, DT shortens much faster than either LVET or PEP, with the latter two variables occupying an ever increasing proportion of the cardiac cycle. Thus, with progressively increasing HR, diastolic filling requires progressively less time time to accomplish the same $\mathrm{SV}$ as does systolic emptying. Hence, at HRmax, in group 20 (192 $\pm 1 \mathrm{bpm})$; LVET required $55 \%$ of the cardiac cycle, while DT occupied only $35 \%$, in group 40 (182 $\pm 1 \mathrm{bpm})$; LVET required $56 \%$ of the cardiac cycle, while DT occupied only $38 \%$, in group 50 (167 $\pm 4 \mathrm{bpm})$; LVET required $55 \%$ of the cardiac cycle, while DT occupied only $37 \%$, and in group 60 (157 $\pm 4 \mathrm{bpm}$ ); LVET required $53 \%$ of the cardiac cycle, while DT occupied only $39 \%$ (Figures 12 and 14). 


\section{Diastolic Filling Rate and Left Yentricular Ejection Rate}

As previously reviewed, since since the last matched HR of all four groups was 150 bpm, CTI could not be statistically compared between groups beyond a HR of $150 \mathrm{bpm}$. Further, since DFR and LVER measurements were calculated from CTI measurements, DFR and LVER could also not be statistically compared between groups beyond a HR of $150 \mathrm{bpm}$.

In Figure 15, DFR is illustrated as a function of HR and group. Analysis of DFR $\left(\mathrm{ml} \cdot \mathrm{s}^{-1}\right)$ revealed a significant main effect for $\mathrm{HR}(\mathrm{F}(3,57)=349.4, \mathrm{p}<0.0001)$. Tukey post-hoc analysis of the mean DFR pooled across groups revealed a significant progressive increase in DFR with each successive increase in HR up to $150 \mathrm{bpm}$. No group differences were observed for DFR, such that age had no significant effect on DFR. Further, the independent analysis of DFR values at $150 \mathrm{bpm}$ revealed no significant group differences (Table 2).

Additionally, although DFRmax is faster in group 20 compared to groups 50 and 60 (1132 $\pm 40 \mathrm{vs} 801 \pm 64$, and $683 \pm 41 \mathrm{ml} \cdot \mathrm{s}^{-1}$, respectively), this is strictly due to the fact that group 20 was at a higher HRmax than groups 50 and 60 (192 \pm 1 vs $167 \pm 4$ bpm and 157 \pm 4 bpm, respectively).

In Figure 16, LVER is illustrated as a function of HR and group. Analysis of LVER $\left(\mathrm{ml} \cdot \mathrm{s}^{-\mathrm{t}}\right.$ ) revealed a significant main effect for $\mathrm{HR}(\mathrm{F}(3,57)=372.9, \mathrm{p}<0.0001)$. Tukey post-hoc analysis of the mean LVER pooled across groups revealed a significant progressive increase in LVER with each successive increase in HR up to $150 \mathrm{bpm}$. No group differences were observed for LVER, such that age had no significant effect on LVER. Further, the independent analysis of LVER values at $150 \mathrm{bpm}$ revealed no significant group differences 


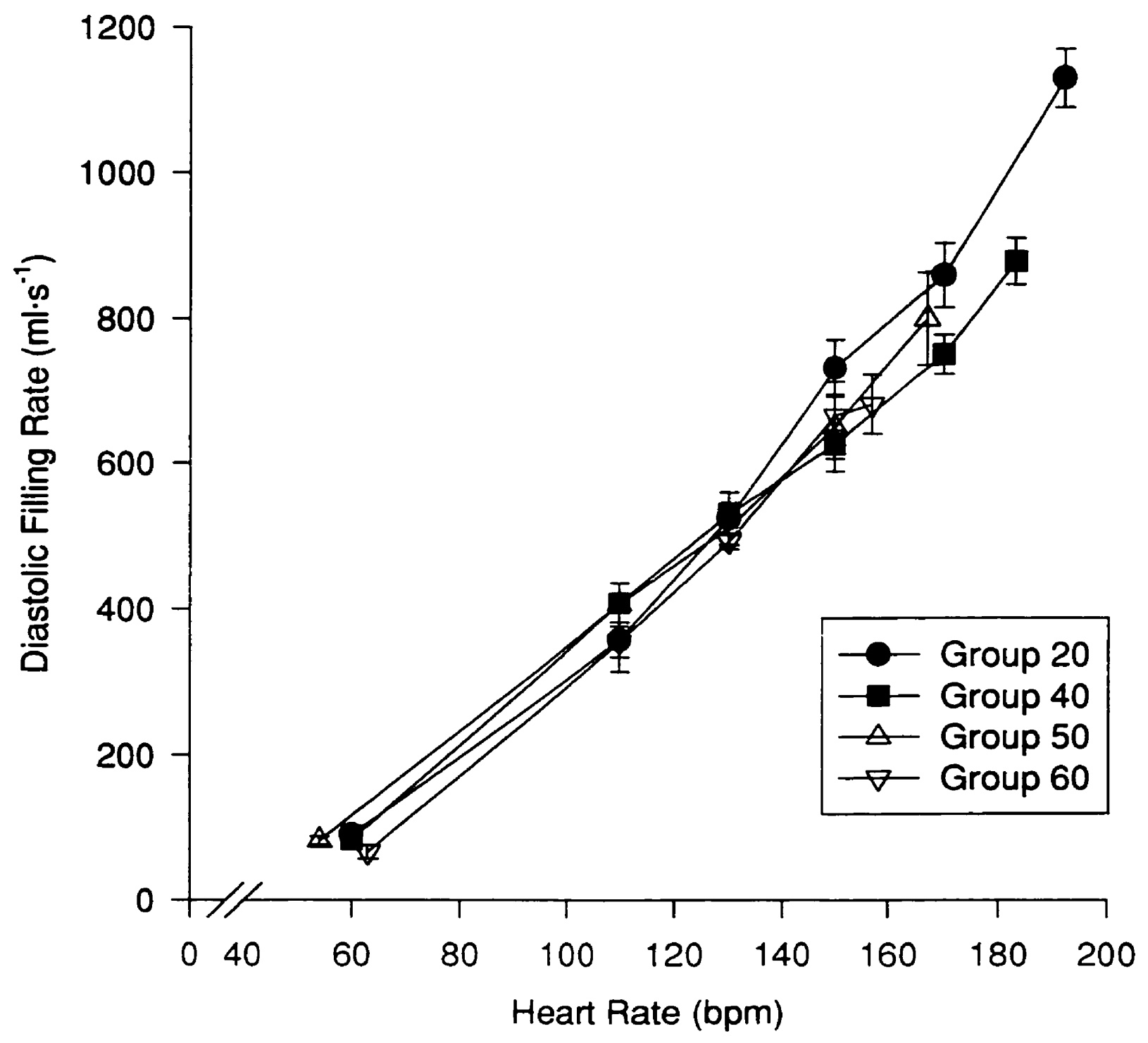

Figure 15. Diastolic filling rate as a function of HR and group. No significant differences between groups. Bars = SEM. 


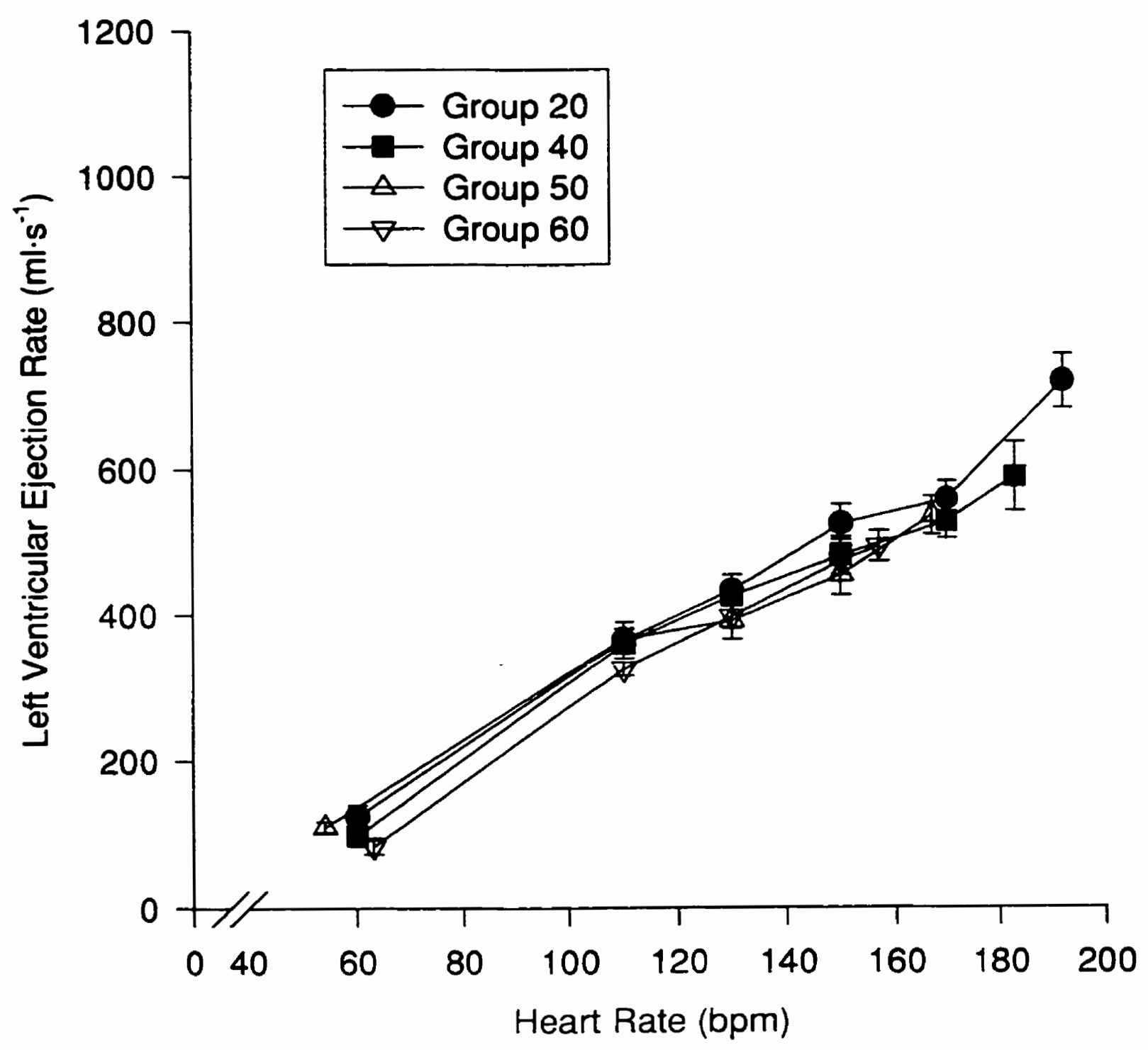

Figure 16. Left ventricular ejection rate as a function of $H R$ and group. No significant differences between groups. Bars $=$ SEM. 
(Table 2).

Additionally, although LVERmax is faster in group 20 compared to groups 50 and 60 (720 \pm 40 vs $537 \pm 26$, and $495 \pm 21 \mathrm{ml} \cdot \mathrm{s}^{-1}$, respectively), this is strictly due to the fact that group 20 was at a higher HRmax than groups 50 and $60(192 \pm 1$ vs $167 \pm 4$ bpm and 157 \pm 4 bpm, respectively).

When comparing the relative contributions of DFR and LVER at matched HR during exercise, DFR was faster than LVER in all four groups. For instance, at $150 \mathrm{bpm}$, in group 20; DFR was $40 \%$ faster than LVER, in group 40; DFR was $30 \%$ faster than LVER, in group 50; DFR was $43 \%$ faster than LVER, and in group 60 DFR was $40 \%$ faster than LVER (Table 2). Further, at HRmax, although the difference was not as impressive in group 60 compared to group 20 (due to a lower HRmax in group 60), DFRmax was faster than LVERmax in all four groups. For instance, in group 20; DFRmax was 57\% faster than LVERmax, in group 40; DFRmax was 49\% faster than LVERmax, in group 50; DFRmax was 49\% faster than LVERmax, and in group 60 DFRmax was 38\% faster than LVERmax (Figures 15 and 16).

\section{Blood Pressure, Mean Arterial Pressure and Total Peripheral Resistance}

In Figure 17, SBP is illustrated as a function of $\mathrm{HR}$ and group. Analysis of SBP ( $\mathrm{mmHg}$ ) revealed a significant interaction effect between $\mathrm{HR}$ and group $(F(15,95)=2.75$, $\mathrm{p}<0.005)$. Tukey post-hoc analysis of the interaction effect showed that SBP increased significantly from rest to $110 \mathrm{bpm}$ in all four groups. Only group 60 showed an increase from $110 \mathrm{bpm}$ to $130 \mathrm{bpm}$. Tukey post-hoc analysis of the interaction effect also revealed that 


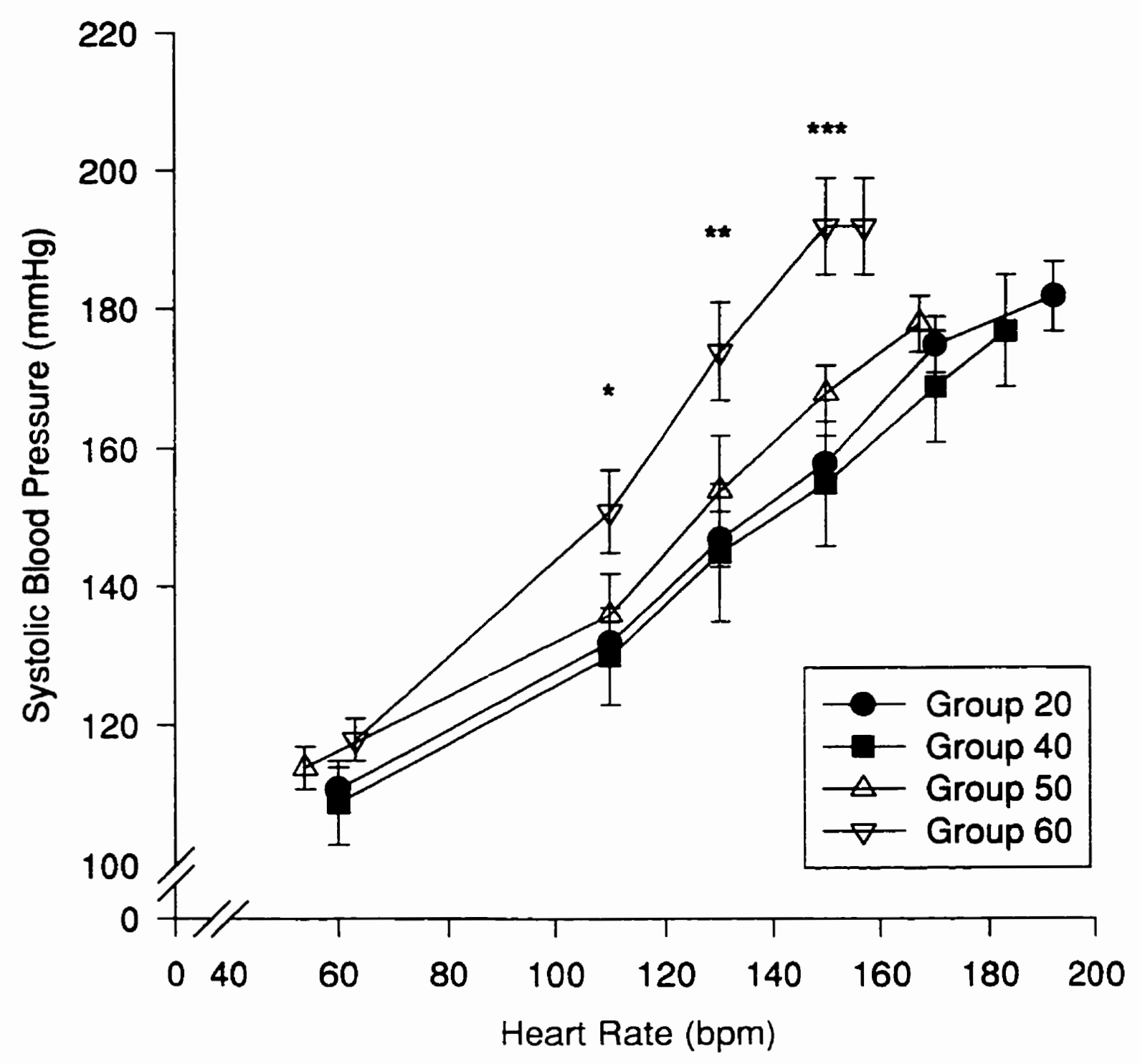

Figure 17. Systolic blood pressure as a function of $H R$ and group. - Group $60>$ Group 40 at 110 bpm ( $<<0.05)$. "* Group $60>$ Groups 20 and 40 at $130 \mathrm{bpm}(p<0.05)$. ${ }^{\star \star \star}$ Group $60>$ than all other groups at $150 \mathrm{bpm}(p<0.05)$. Bars $=$ SEM. 
group 60 had a significantly higher SBP at 110 bpm than group 40 (16\%) (151 \pm 6 vs $130 \pm$ $7 \mathrm{mmHg}$ ). Further, at $130 \mathrm{bpm}$ group 60 had a significantly higher SBP than groups 20 (18\%) and $40(20 \%)(174 \pm 7$ vs $147 \pm 4$ and $145 \pm 10 \mathrm{mmHg}$, respectively). In addition group 60 had a significantly higher SBP at $150 \mathrm{bpm}$ than groups 20 (21\%), 40 (24\%), and 50 (14\%) (192 \pm 7 vs $158 \pm 4,155 \pm 9$ and $168 \pm 4 \mathrm{mmHg}$, respectively). No other group differences were observed for SBP at any of the other target HR. The independent analysis of SBP values at max revealed no significant differences between groups.

Since the older individuals were exercising at a higher relative intensity than the younger individuals at any given $\mathrm{HR}$, higher $\mathrm{BP}$ responses would be expected to be observed in the older individuals. Thus, it has been suggested that older and younger individuals be compared at the same relative percentage of maximal oxygen uptake, since it appears to be the primary determinant of the arterial pressure response to exercise (10). In Figure 18, SBP is depicted as a function of the percentage of maximal oxygen uptake and group. Analysis of SBP (mmHg) revealed a significant main effect for the percentage of maximal oxygen uptake $(F(3,57)=180.5, p<0.0001)$. Tukey post-hoc analysis of the mean SBP pooled across groups revealed that SBP was significantly different at all relative percentages of maximal oxygen uptake, such that it increased progressively with each increment in work rate up to maximum. No group differences were observed for SBP when expressed relative to the percentage of maximal oxygen uptake, such that age had no significant effect on SBP.

In Figure 19, DBP is illustrated as a function of HR and group. Analysis of DBP (mmHg) revealed a significant main effect for $\operatorname{HR}(F(5,95)=2.78, p<0.05)$. Tukey post-hoc 


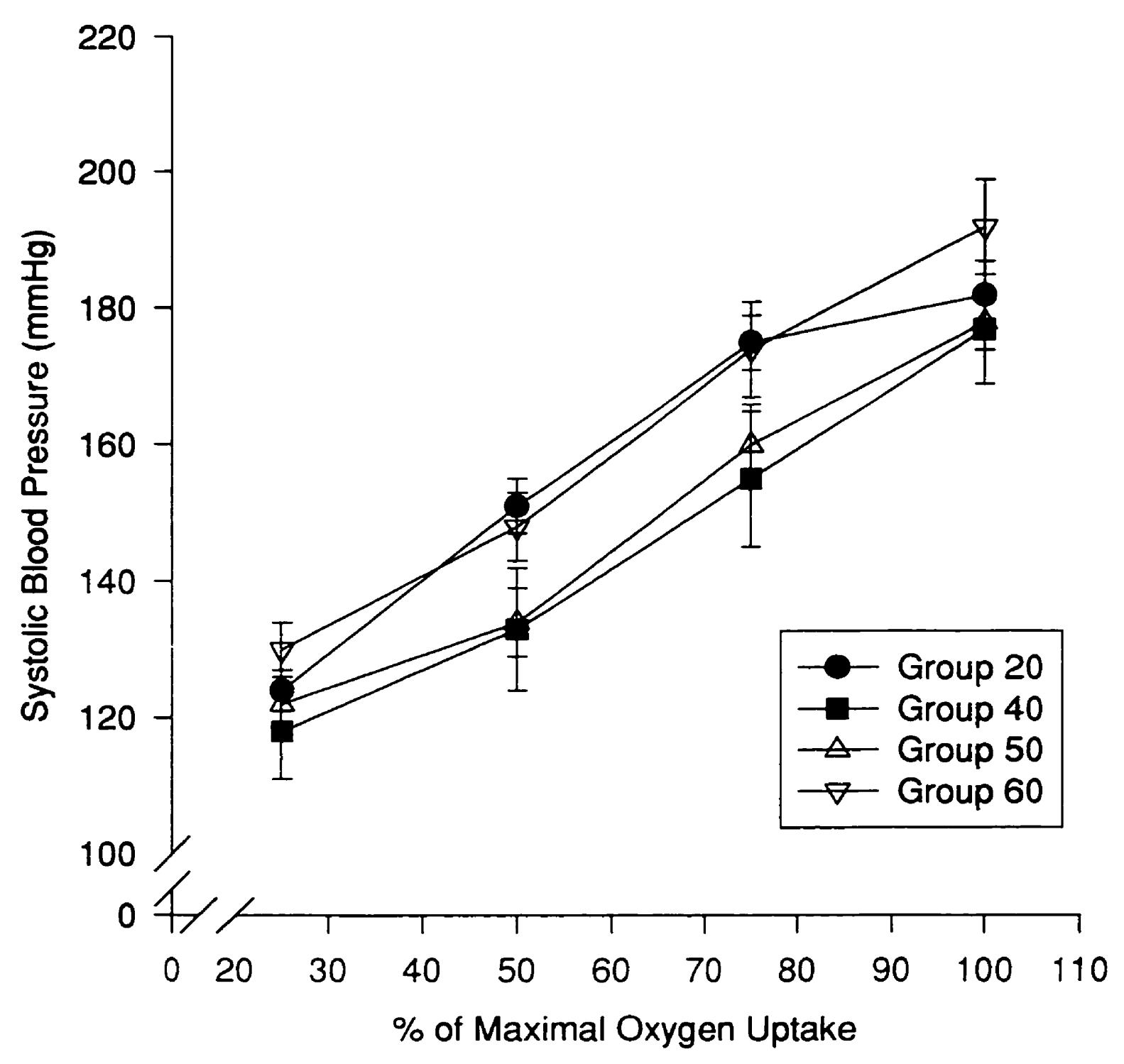

Figure 18. Systolic blood pressure as a function of the percentage of maximal oxygen uptake and group. No significant differences between groups. Bars $=$ SEM. 


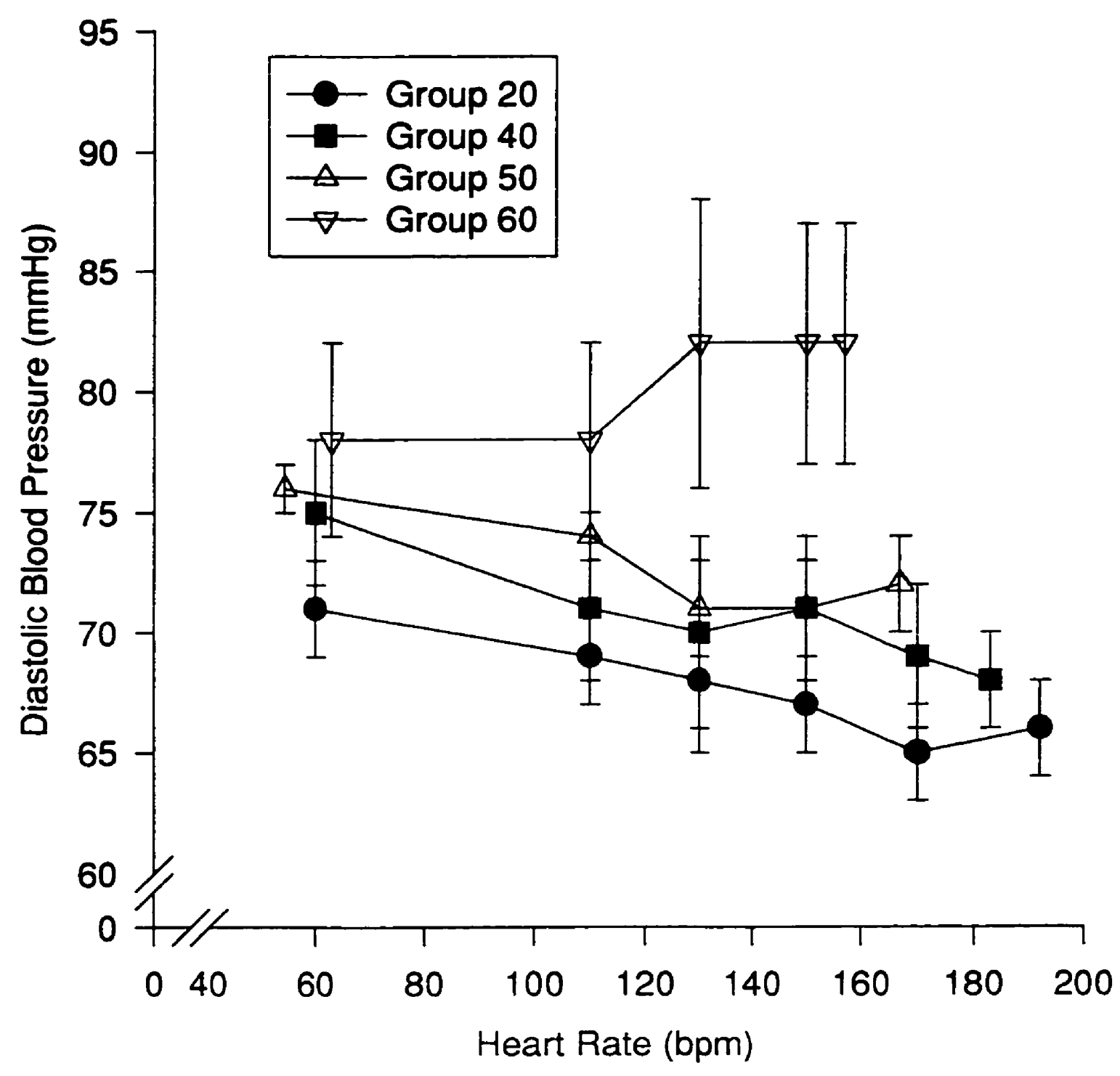

Figure 19. Diastolic blood pressure as a function of HR and group. No significant differences between groups. Bars $=$ SEM. 
analysis showed that when DBP was pooled across groups, a significant decrease was observed from rest to $170 \mathrm{bpm}$. No other significant differences were observed for this effect. A significant main effect for group was also observed $(F(3,19)=3.80, p<0.05)$. Tukey posthoc analysis of the mean DBP pooled across HR, revealed that group 60 had a significantly higher DBP than group 20 (80 \pm 4 vs $68 \pm 2 \mathrm{mmHg}$ ). No other significant group differences were observed for this effect. The independent analysis of DBP values at max revealed no significant differences between groups.

In Figure 20, DBP is depicted as a function of the percentage of maximal oxygen uptake and group. Analysis of DBP ( $\mathrm{mmHg}$ ) revealed a significant main effect for group $(F(3,19)=4.09, p<0.05)$. Tukey post-hoc analysis of the mean DBP pooled across the percentages of maximal oxygen uptake revealed that DBP was significantly higher in group 60 compared to group 20.

In Figure 21, MAP is illustrated as a function of HR and group. Analysis of MAP (mmHg) revealed an interaction effect between HR and group $(F(15,95)=1.87, p<0.05)$. Tukey post-hoc analysis of the interaction effect showed that MAP increased significantly from rest to $110 \mathrm{bpm}$ and from $110 \mathrm{bpm}$ to $130 \mathrm{bpm}$ only in group 60 . Tukey post-hoc analysis of the interaction effect also revealed that group 60 had a significantly higher MAP at $110 \mathrm{bpm}$ than groups $20(14 \%)$ and $40(12 \%)(102.2 \pm 4.3$ vs $89.9 \pm 2.1$ and $91.0 \pm 4.4$ mmHg, respectively). At $130 \mathrm{bpm}$, group 60 had a significantly higher MAP than groups 20 (19\%), 40 (18\%) and $50(14 \%)(112.5 \pm 5.4$ vs $94.1 \pm 2.3,95.4 \pm 5.3$ and $99.0 \pm 2.9 \mathrm{mmHg}$, respectively). In addition, at $150 \mathrm{bpm}$, group 60 had a significantly higher MAP than groups 


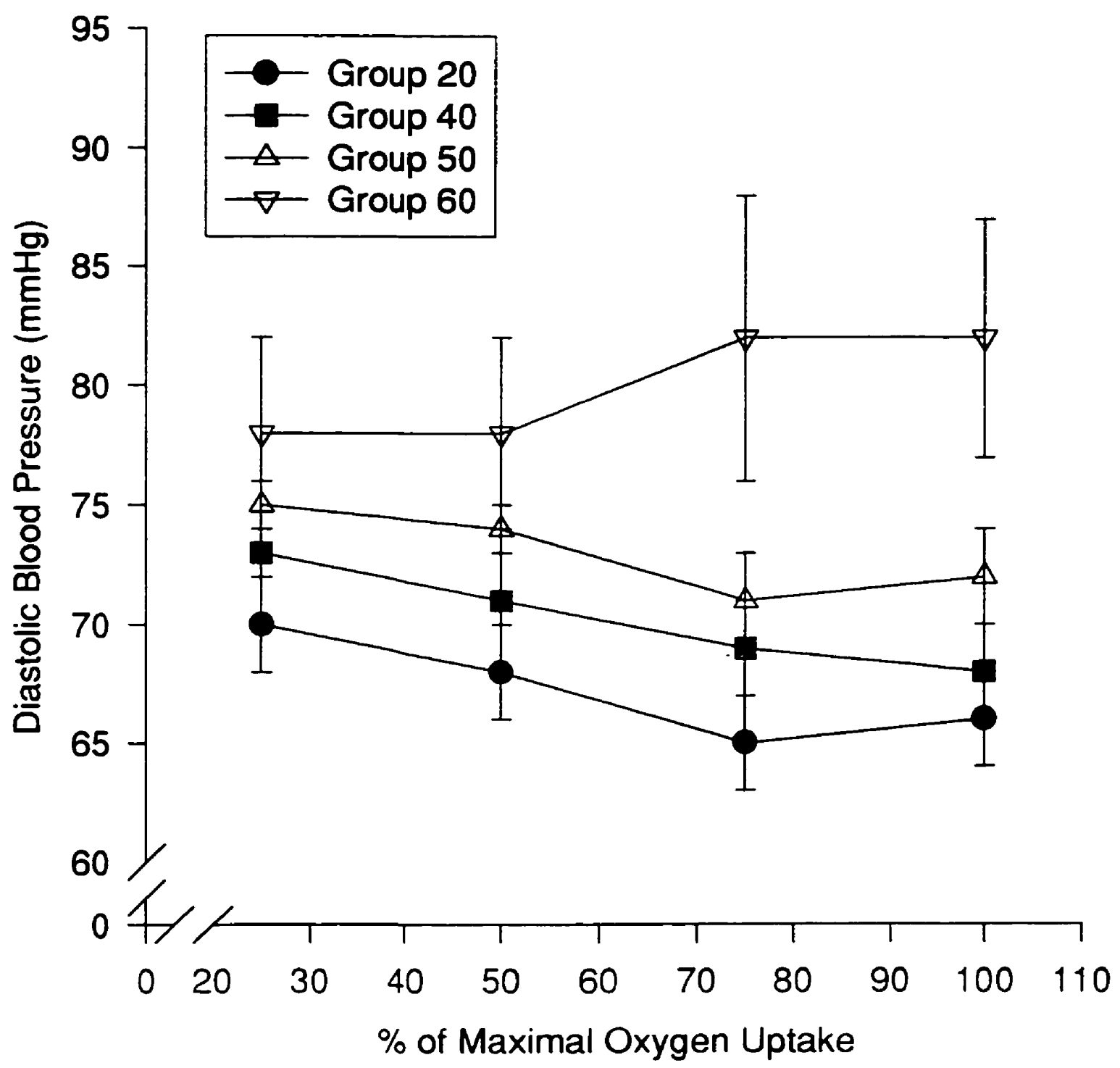

Figure 20. Diastolic blood pressure as a function of the percentage of maximal oxygen uptake and group. No significant differences between groups. Bars = SEM. 


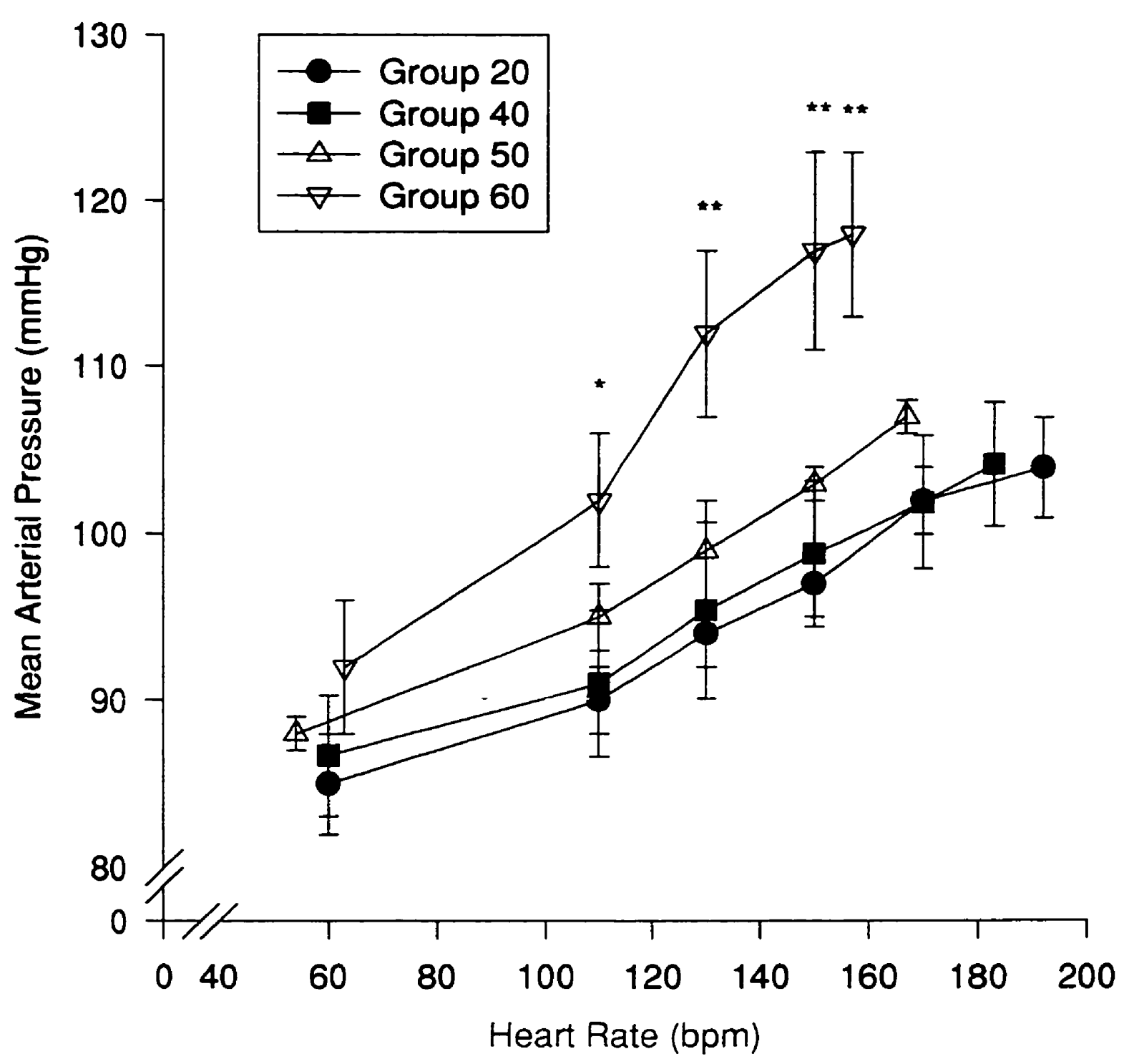

Figure 21. Mean arterial pressure as a function of HR and group.

- Group $60>$ Groups 20 and 40 at 110 bpm. "* Group $60>$ all other Groups at $130 \mathrm{bpm}, 150 \mathrm{bpm}$ and max. Bars = SEM. 
$20(20 \%), 40(18 \%)$ and $50(13 \%)(116.7 \pm 5.8$ vs $97.4 \pm 1.9,98.8 \pm 4.4$ and $103.0 \pm 1.0$ mmHg, respectively). Further, at max, group 60 had a significantly higher MAP than groups $20(13 \%), 40(13 \%)$ and $50(10 \%)$ (Table 2). No other significant group differences were observed for this effect. The independent analysis of MAP values at max revealed no significant group differences.

In Figure 22, MAP is depicted as a function of the percentage of maximal oxygen uptake and group. Analysis of MAP (mmHg) revealed a significant main effect for the percentage of maximal oxygen uptake $(F(3,57)=97.9, p<0.0001)$. Tukey post-hoc analysis of the mean MAP pooled across groups revealed that MAP was significantly different at all relative percentages of maximal oxygen uptake, such that it increased progressively with each increment in work rate up to maximum. No group differences were observed for MAP when expressed relative to the percentages of maximal oxygen uptake, such that age had no significant effect on MAP.

In Figure 23, TPR is illustrated as a function of $H R$ and group. Analysis of TPR $\left(\mathrm{mmHg} \cdot \mathrm{l}^{-1} \cdot \mathrm{min}^{-1}\right)$ revealed a significant main effect for $H R(F(5,95)=220.3, \mathrm{p}<0.0001)$. Tukey post-hoc analysis of the mean TPR pooled across groups, revealed a significant decrease from rest to $110 \mathrm{bpm}$. A significant main effect for group was also observed $(F(3,19)=5.36$, $\mathrm{p}<0.01$ ). Tukey post-hoc analysis of the mean TPR pooled across HR, revealed that group 60 had a significantly higher TPR than group $20\left(14.1 \pm 1.1 \mathrm{vs} 10.2 \pm 0.82 \mathrm{mmHg} \cdot \mathrm{l}^{-1} \cdot \mathrm{min}^{-1}\right.$, $\mathrm{p}<0.01$ ). No other significant group differences were observed for this effect. The independent analysis of TPR values at $\max (F(3,19)=16.61, p<0.0001)$ revealed that the 


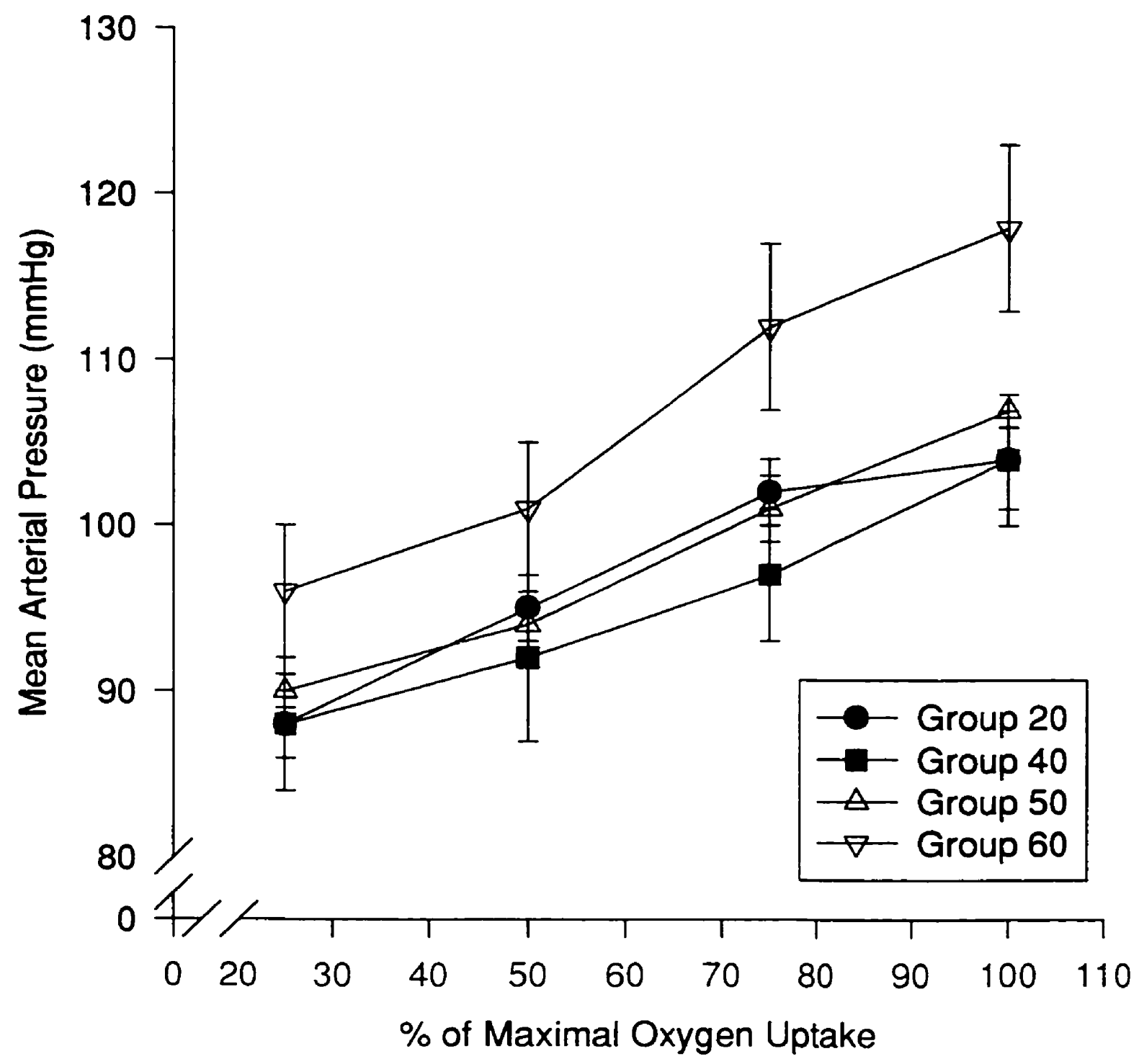

Figure 22. Mean arterial pressure as a function of the percentage of maximal oxygen uptake and group. No significant differences between groups. Bars = SEM. 


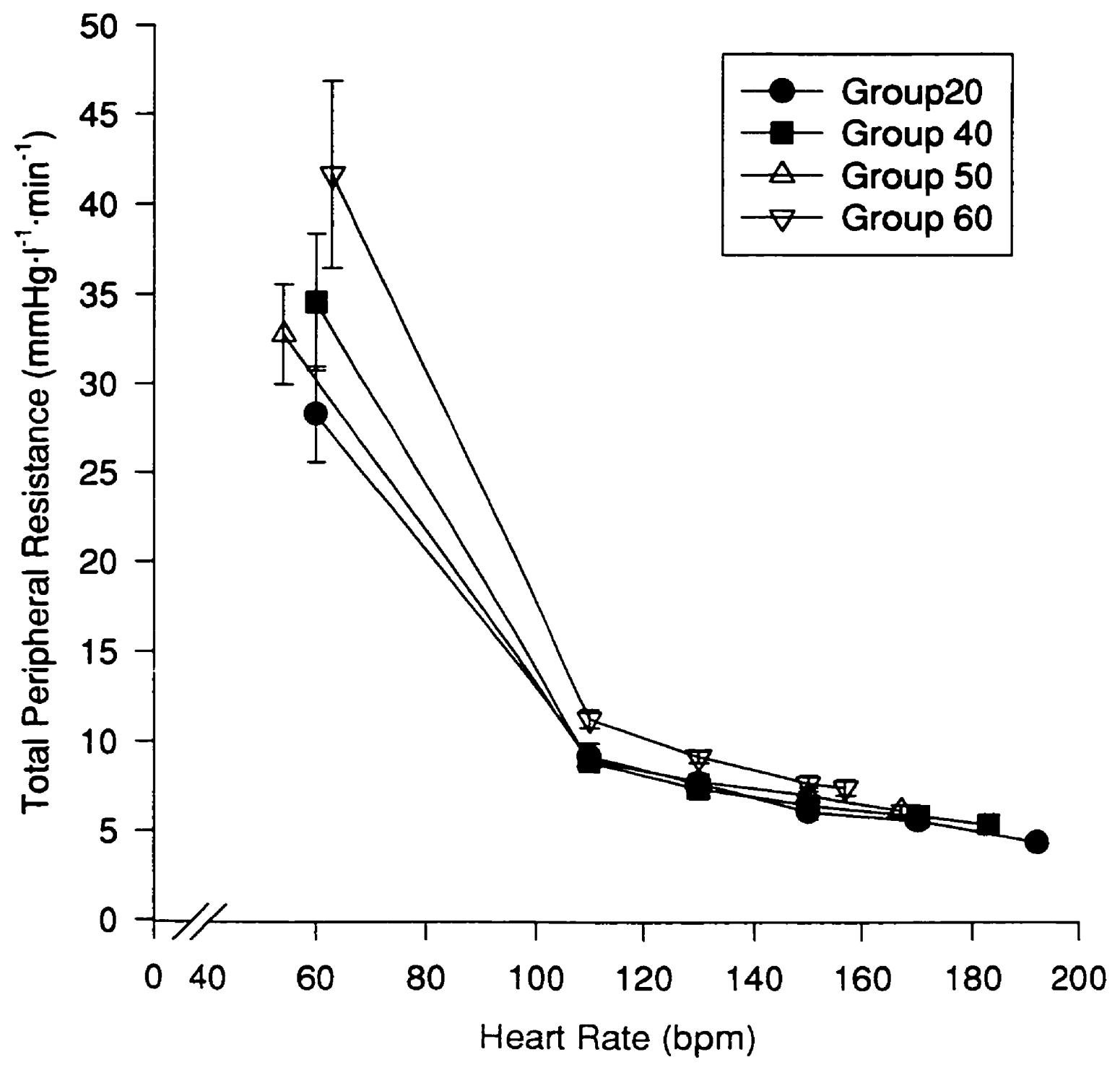

Figure 23. Total peripheral resistance as a function of HR and group. No significant differences between groups. Bars = SEM. 
TPRmax $\left(\mathrm{mmHg} \cdot \mathrm{l}^{-1} \cdot \mathrm{min}^{-1}\right)$ was significantly higher in groups $50(41 \%)$ and $60(71 \%)$ compared to group 20 and in group 60 (39\%) compared to group 40 (Table 2). In Figure 24, TPR is illustrated as a function of the percentage of maximal oxygen uptake and group. Analysis of TPR $\left(\mathrm{mmHg} \cdot \mathrm{l}^{-1} \cdot \mathrm{min}^{-1}\right)$ revealed a significant interaction effect between the percentage of maximal oxygen uptake and group $(F(9,57)=5.36, p<0.0001)$. Tukey post-hoc analysis of the interaction effect revealed that TPR decreased significantly from $25 \%$ to $50 \%$ of maximal oxygen uptake in all groups. Tukey post-hoc analysis of the interaction effect also revealed that at $25 \%$ of maximal oxygen uptake, significantly higher TPR were observed in groups $40(49 \%), 50(58 \%)$ and $60(99 \%)$, compared to group 20 . In addition, at $25 \%$ of maximal oxygen uptake, a significantly higher TPR was observed in group 60 compared to groups $40(33 \%)$ and $50(26 \%)$. Further at $50 \%$ of maximal oxygen uptake, significantly higher TPR were observed in groups 40 (28\%), 50 (68\%) and 60 (89\%), compared to group 20. 


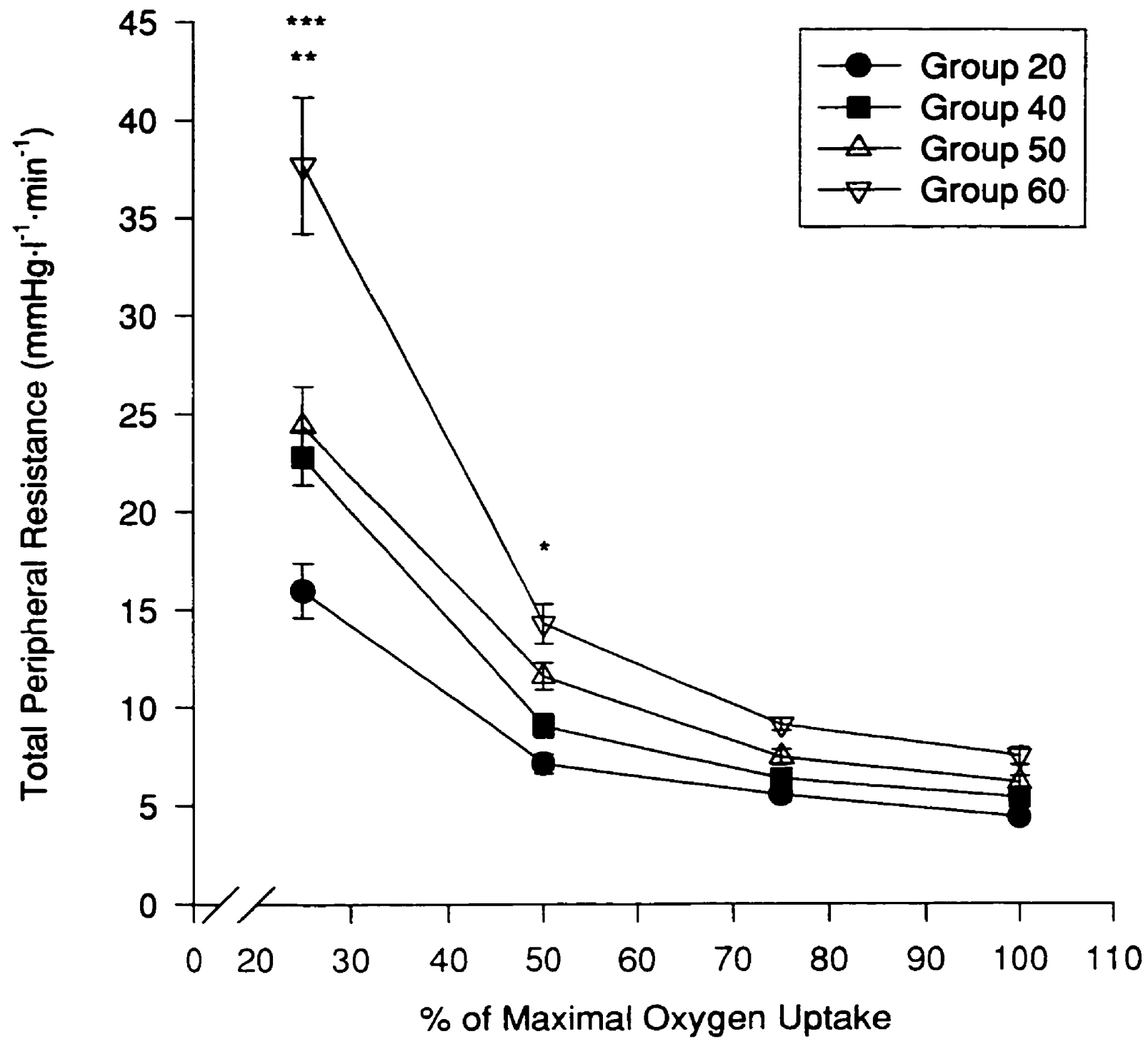

Figure 24. Total peripheral resistance as a function of the percentage of maximal oxygen uptake and group. *** Groups 40,50 and $60>$ Group 20 at $25 \%$. * Group $60>$ Groups 40 and 50 at 25\%. * Group $60>$ Group 20 at $50 \%$ (all $p<0.05$ ). Bars $=$ SEM. 


\section{SUMMARY OF RESULTS}

The components of cardiac function of primary interest in the present investigation were a- $\overline{\mathrm{v}} \mathrm{O}_{2}, \mathrm{Q}, \mathrm{SV}, \mathrm{BV}$, the CTI (LVET, PEP, DT), DFR, LVER, BP, MAP and TPR. These components were examined at rest and during incremental exercise to maximum, in ET females ages 20-29 yr (group 20), 40-45 yr (group 40), 49-54 yr (group 50) and 58-63 yr (group 60). Throughout incremental work rates to maximum, age-related differences were observed for $\mathrm{VO}_{2}, \mathrm{Q}, \mathrm{SBP}, \mathrm{DBP}, \mathrm{MAP}$ and TPR. Whereas, no significant age-related differences were observed for a-viDO ${ }_{2}, \mathrm{SV}, \mathrm{BV}, \mathrm{LVET}, \mathrm{DT}$, PEP, DFR or LVER.

In comparison to group $20, \mathrm{VO}_{2} \max \left(1 \cdot \mathrm{min}^{-1}\right)$ was significantly lower in groups 40 (17\%), $50\left(26 \%\right.$ ) and $60\left(35 \%\right.$ ), such that it declined by $\sim 0.035 \mathrm{l} \cdot \mathrm{min}^{-1} \cdot \mathrm{yr}^{-1}$ (or $\sim 0.51 \mathrm{ml} \cdot$ $\mathrm{kg}^{-1} \cdot \mathrm{min}^{-1} \cdot \mathrm{yr}^{-1}, 8 \%$ decline per decade) between the average ages of 22 and $60 \mathrm{yr}$. The lower $\nabla_{2} \max \left(1 \cdot \min ^{-1}\right)$ was due to a significantly lower $Q \max \left(1 \cdot \mathrm{min}^{-1}\right)$ in groups $40(19 \%), 50$ (26\%) and 60 (34\%) compared to group 20 (with a decline of $\sim 0.22 \mathrm{l} \cdot \mathrm{min}^{-1} \cdot \mathrm{yr}^{-1}$ ), whereas no significant age difference was observed for a- $\overrightarrow{\mathrm{v} D O} \mathrm{O}_{2} \max \left(\mathrm{ml} \mathrm{O}_{2} \cdot 100 \mathrm{ml}^{-1}\right)$. The smaller $\mathrm{Q} \max$ $\left(1 \cdot \mathrm{min}^{-1}\right)$ was due to a significantly lower HRmax (bpm) in groups $50(13 \%)$ and $60(18 \%)$ compared to group 20 and in groups $50(8 \%)$ and $60(14 \%)$ compared to group 40 (with a decline of $\sim 0.92 \mathrm{bpm} \cdot \mathrm{yr}^{-1}$ ), whereas no significant age difference was observed for $S V \max$ $(\mathrm{ml})$, or for $\mathrm{BV}$ in $\mathrm{ml}$ or $\mathrm{ml} \cdot \mathrm{kg}^{-1}$.

At matched HR during exercise, the duration of LVET was greater than the duration of DT and DFR was faster than LVER in all four groups, suggesting diastolic function is the major determinant of an enhanced cardiac function. The MAPmax (mmHg) was significantly 
higher in group 60 compared to groups $20(13 \%), 40$ (13\%) and 50 (10\%). One mechansim for the higher MAPmax was the significantly higher TPRmax $\left(\mathrm{mmHg} \cdot \mathrm{l}^{-1} \cdot \mathrm{min}^{-1}\right)$ in groups 50 (41\%) and $60(71 \%)$ compared to group 20, and in group 60 (39\%) compared to group 40 (Table 2). 


\section{CHAPTER V}

\section{DISCUSSION}

The purpose of this investigation was to examine the cardiac function of ET females, as a function of aging, at rest and during incremental exercise to maximum. The components of cardiac function of primary interest were Q, SV, BV, CTI (LVET, PEP, DT), DFR, LVER, BP, MAP and TPR. To determine whether any changes occur as a function of aging, these components were examined and compared between ET females 20-29 yr (group 20), 40-45 yr (group 40), 49-54 yr (group 50) and 58-63 yr (group 60). The majority of the agerelated differences in cardiac function did not reach statistical significance until the participants were exercising maximally.

In addition, gender related differences in cardiac function were examined by comparing the youngest grcup of ET females $(22.4 \pm 1.4 \mathrm{yr})$ to that of an age-matched group of ET males (22.1 $\pm 1.1 \mathrm{yr})$ who were previously examined from the same laboratory (289). The present investigation represents the first to examine cardiac function in ET females via simultaneous recordings of CTI and SV.

\section{Maximal Oxygen Uptake in ET Individuals and the Relationship to Age and Gender}

It is well known that $\mathrm{ET}$ individuals have a significantly larger $\mathrm{VO}_{2} \max$ than $\mathrm{NA}$ individuals, regardless of age or gender. For instance, investigators have reported an average $\mathrm{VO}_{2} \max$ of $\sim 55-62 \mathrm{ml} \cdot \mathrm{kg}^{-1} \cdot \mathrm{min}^{-1}$ for ET females $18-30 \mathrm{yr}(52,98,181,205,255,280,306$, 319), an average of $\sim 47 \mathrm{ml} \cdot \mathrm{kg}^{-1} \cdot \mathrm{min}^{-1}$ for ET females $40-44 \mathrm{yr}$, an average of $\sim 44 \mathrm{ml} \cdot \mathrm{kg}^{-1} \cdot \mathrm{min}^{-1}$ for ET females $45-49 \mathrm{yr}$, and an average of $\sim 40 \mathrm{ml} \cdot \mathrm{kg}^{-1} \cdot \mathrm{min}^{-1}$ for ET females 50-70 $\mathrm{yr}$ (295). 
In the present investigation, group 20 had a mean $\mathrm{VO}_{2} \max$ of $63.7 \pm 2.3 \mathrm{ml} \cdot \mathrm{kg}^{-1} \cdot \mathrm{min}^{-1}$, group 40 had a mean of $51.0 \pm 3.1 \mathrm{ml} \cdot \mathrm{kg}^{-1} \cdot \mathrm{min}^{-1}$, group 50 had a mean of $47.0 \pm 2.5 \mathrm{ml} \cdot \mathrm{kg}^{-1} \cdot \mathrm{min}^{-1}$ and group 60 , had a mean of $44.2 \pm 1.7 \mathrm{ml} \cdot \mathrm{kg}^{-1} \cdot \mathrm{min}^{-1}$. The number of participants who were able to reach their age-predicted HRmax did not differ between groups $(5 / 6,6 / 6,5 / 6$ and $4 / 5$ for groups $20,40,50$ and 60 , respectively), thus providing support that a true $\mathrm{VO}_{2}$ max was attained by all participants.

\section{Age-Related Changes In $\mathrm{VO}_{2} \max$}

It has been well documented that $\mathrm{VO}_{2} \max$ declines progressively with advancing age, regardless of gender or training status. In absolute terms $\mathrm{VO}_{2} \max \left(1 \cdot \mathrm{min}^{-1}\right)$ was $1.7 \%$ lower in group 40, 26\% lower in group 50 and $35 \%$ lower in group 60 compared to group 20. Additionally, $\mathrm{VO}_{2} \max$ was significantly lower in groups $50(10 \%)$ and $60(21 \%)$ compared to group 20 (refer to Figure 5 and Table 2). The $26 \%$ lower $\mathrm{VO}_{2} \max$ in group $50(51 \pm 0.8$ yr) compared to group $20(22 \pm 1 \mathrm{yr}$ ), is similar to the results of Ogawa et al. (205), who observed a $28 \%$ lower absolute $\mathrm{VO}_{2} \max$ in older ET females (57 $\pm 3 \mathrm{yr}$ ) compared to younger ET females ( $26 \pm 3 \mathrm{yr}$ ). When expressed relative to body mass, group 20 still had a significantly larger $\mathrm{VO}_{2} \max$ than all other groups, although the difference between groups 40 and 60 was no longer significant. No other group differences existed for $\mathrm{VO}_{2}$ at any of the other submaximal HR. In agreement with the $0.47 \mathrm{ml} \cdot \mathrm{kg}^{-1} \cdot \mathrm{min}^{-1} \cdot \mathrm{yr}^{-1}$ decline in the $\mathrm{VO}_{2}$ max in female masters runners ages 35 to $70 \mathrm{yr}$, reported by Wells et al. (295), the ET females in this investigation showed a decline in $\mathrm{VO}_{2} \max$ of $0.58 \mathrm{ml} \cdot \mathrm{kg}^{-1} \cdot \mathrm{min}^{-1} \cdot \mathrm{yr}^{-1}$ between ages 22 to 63 yr. Possible contributing factors to the age-related decline in $\mathrm{VO}_{2} \max$ are 
differences in body mass, body composition, hormonal status, Qmax, SVmax, HRmax and $\mathrm{a}-\overline{\mathrm{vDOO}} \mathbf{2}_{\mathbf{m a x}}$

i) Age-related changes in body mass and body composition

In contrast to the observations of some investigators $(62,122,214,218,276)$, the ET females did not show a decline in LBM, an increase in relative fat percentage or a change in body mass as a function of aging. However, in agreement with some other researchers (122, 180), the ET females maintained their LBM, their relative fat percentage, as well as their body mass. Thus, body mass and body composition did not contribute significantly to the agerelated decline in $\mathrm{VO}_{2} \max$ in the ET females from the present investigation.

\section{ii) Hormonal status}

Although there is no conclusive evidence to date showing that $\mathrm{VO}_{2} \max$ is significantly affected by the phase of the menstrual cycle, variations in PV which occur during the menstrual cycle (due to fluid shifts), have been shown to affect cardiovascular and thermoregulatory mechanisms and BV plays a major role in cardiac function $(119,152)$. Thus, precaution was taken to control for fluid shifts, such that all premenopausal females were tested during the follicular phase of their menstrual cycle (1-8 days post-menses). Additionally, although menopausal status has not been shown to have a significant influence on $\mathrm{VO}_{2} \max (265,295)$, the follicular phase was chosen for testing since postmenopausal females display similar levels of estrogen and progesterone as regularly menstruating females who are in this phase (271). Further, since there is no conclusive evidence to date showing that the use of OCA or HRT has a significant influence on $\mathrm{VO}_{2} \max (17,50,187,202)$, 
participants using these medications were not eliminated from the present investigation.

Group 20 had two OCA users, group 40 had one and group 50 had no OCA users. Four participants in group 50 were postmenopausal and one was using HRT. All five participants in group 60 were postmenopausal, and none were using HRT. In agreement with the literature, when the three OCA users were compared to the eleven premenopausal females not using OCA, $\mathrm{VO}_{2} \max$ in $1 \cdot \mathrm{min}^{-1}$ and $\mathrm{ml} \cdot \mathrm{kg}^{-1} \cdot \mathrm{min}^{-1}$ did not differ between groups. However, when the eight post-menopausal females (not using HRT) were compared to the eleven premenopausal females (not using OCA) a significant difference existed for $\mathrm{VO}_{2}$ max in $1 \cdot \mathrm{min}^{-1}$ and $\mathrm{ml} \cdot \mathrm{kg}^{-1} \cdot \mathrm{min}^{-1}$. The mechanism responsible for this difference was a lower $\mathrm{Qmax}$ in the postmenopausal compared to the premenopausal females, while a-vDO ${ }_{2}$ max did not differ between groups. The mechanism responsible for the lower Qmax observed in the postmenopausal females was a lower HRmax, while SVmax was not different. The reason for the lower HRmax was a significant difference in age of the premenopausal compared to the postmenopausal females $(36.3 \pm 3.5 \mathrm{vs} 56.7 \pm 1.0 \mathrm{yr}$, respectively). The roles that the other variables (Qmax, SVmax, BV, $\mathrm{HRmax}$ and a-vDO ${ }_{2} \max$ ) play in the age-related decline in $\mathrm{VO}_{2} \mathrm{max}$ will be discussed later in this chapter.

\section{Gender Differences in $\mathrm{VO}_{2} \max$}

Generally, ET males have a larger $\mathrm{VO}_{2} \mathrm{max}$ in $1 \cdot \mathrm{min}^{-1}$ and $\mathrm{ml} \cdot \mathrm{kg}^{-1} \cdot \mathrm{min}^{-1}$, which appears to be maintained as a function of age $(117,122,138,205,265)$. For instance, Stevenson et al. (265) reported that ET females have an $\sim 13-15 \%\left(9-10 \mathrm{ml} \cdot \mathrm{kg}^{-1} \cdot \mathrm{min}^{-1}\right)$ lower $\mathrm{VO}_{2}$ max than ET males, regardless of age (265). In the present investigation, group 20 had a $21 \%$ lower 
absolute $\mathrm{VO}_{2} \max \left(3.84 \pm 0.14\right.$ vs $\left.4.84 \pm 0.10 \mathrm{l} \cdot \mathrm{min}^{-1}\right)$ and when expressed relative to body mass an $8 \%$ bwer $\mathrm{VO}_{2} \max \left(63.7 \pm 2.3 \mathrm{vs} 69.1 \pm 0.9 \mathrm{ml} \cdot \mathrm{kg}^{-1} \cdot \mathrm{min}^{-1},\right)$ than the age-matched ET males from the Warburton et al. (289) study.

Mechanisms Responsible for the Larger $\mathrm{VO}_{2}$ max in ET Males Compared to Females

Mechanisms responsible for the gender-related difference in $\mathrm{VO}_{2} \mathrm{max}$ in ET individuals are: differences in body mass and body composition, $[\mathrm{Hb}]$ and thus, arterial oxygen carrying capacity and transport, fluid shifts during the menstrual cycie, Qmax, SVmax, BV and a$\mathrm{vDO}_{2} \max$.

i) Gender differences in body mass and body composition

Group 20 was $\sim 10 \mathrm{~kg}$ lighter in body mass $(60.3 \pm 1.9 \mathrm{vs} 70.1 \pm 1.6 \mathrm{~kg})$ than the ET males from the Warburton et al. (289) investigation. This finding is in line with the finding of another investigator who observed that female distance-runners were $\sim 13 \cdot \mathrm{kg}$. lighter in total body mass compared to male distance runners (305). The influence of differences in body mass was shown earlier in this discussion, such that when $\mathrm{VO}_{2} \max \left(1 \cdot \mathrm{min}^{-1}\right)$ was expressed relative to body mass, the gender related difference was reduced by $13 \%$ (from 21 to $8 \%$ ). In addition, ET females tend to have a lower $\operatorname{LBM}(31,296)$, and a higher relative body fat percentage than ET males $(80,296,305)$, which in turn contributes to their lower $\mathrm{VO}_{2}$ max in $1 \cdot \mathrm{min}^{-1}$ and $\mathrm{ml} \cdot \mathrm{kg}^{-1} \cdot \mathrm{min}^{\mathrm{t}}$, respectively. In agreement with reported values for female distance runners and cyclists from other investigations $(20,306)$, group 20 had an average LBM of $51 \mathrm{~kg}$ and an average relative body fat of $15.1 \%$. Since the ET females had their fat percentage measured with a different skinfold method (refer to Appendix E) than the ET 
males in the Warburton et al. (289) investigation, comparison between the genders in relative body fat percentage and thus, LBM could not be made.

ii) Gender differences in $\left[\mathrm{Hb}, \mathrm{O}_{2}\right.$ carrying capacity and $\mathrm{O}_{2}$ transport

In addition, [Hb] is lower in females compared to males, regardless of training status or age $(7,8,91,106)$. An average $[\mathrm{Hb}]$ for a female is $13.9 \mathrm{~g} \cdot 100 \mathrm{~m}^{-1}$, while an average for a male is $15.8 \mathrm{~g} \cdot 100 \mathrm{ml}^{-1}$ (106). Group 20 had a $6 \%$ lower [Hb] than the ET males in the Warburton et al investigation (289) $\left(14.4 \pm 0.3 \mathrm{vs} 15.3 \pm 0.3 \mathrm{~g} \cdot 100 \mathrm{ml}^{-1}\right)$. Assuming that each gram of haemoglobin can carry $1.39 \mathrm{ml}$ of oxygen per $100 \mathrm{ml}$ of blood (299), this results in a lower oxygen content of arterial blood in the ET females, compared to the ET males (20.0 \pm 0.4 vs $\left.21.3 \pm 0.4 \mathrm{ml} \mathrm{O}_{2} \cdot 100 \mathrm{ml}^{-1}\right)$. A lower oxygen content, in combination with a lower $Q \max \left(24.1 \pm 0.9\right.$ vs $30.0 \pm 0.61 \cdot \mathrm{min}^{-1}$ ) $)$ in the ET females compared to the ET males results in less oxygen being available for transport to the exercising muscles during maximal exercise $\left(4.60 \pm 0.22\right.$ vs $\left.6.42 \pm 0.151 \mathrm{O}_{2} \cdot \mathrm{min}^{-1}\right)$, thus potentially contributing to a lower a$\mathrm{vDO}_{2} \max$ and thus $\mathrm{VO}_{2} \max$ in the ET females.

iii) Influence of the menstrual cycle

Since males and females have been shown to display similar PV shifts during exercise when females are examined during the follicular phase of their menstrual cycle (96), this phase was chosen for testing, in an attempt to control for fluid shifts during the menstrual cycle.

The other mechanisms which may contribute to the gender differences in $\mathrm{VO}_{2} \max$ (Qmax, SVmax, BV and a- $\mathrm{vDO}_{2} \max$ ) will be discussed later in this chapter. 


\section{Cardiac Output in ET Individuals and the Relationship to Age and Gender}

In ET individuals, a larger Qmax has been observed in both younger and older ET males $(7,11,67,68,82,105,117,122,152,205,222,234,245,308,309)$ as well as in both younger and older ET females (205), compared to age and gender-matched NA individuals. Increases in Qmax may result from increases in $S V \max$ and/or $H R \max$, since $Q=S V \times H R$. Since most researchers have observed no differences in HRmax of ET and NA age-matched males and females $(11,83,117,122,205)$, the mechanism responsible for a larger Qmax is a larger SVmax in ET compared to NA age-matched males and females $(7,11,67,68,82$, $105,117,122,152,205,222,234,245,308,309$ ).

\section{Age-Related Changes in Q}

Controversy exists regarding the age-related changes in Qmax. While some inivestigators have observed an age-related reduction in $Q \max (81,110,117,122,124,139$, $205,222,267,318)$ others have observed no change in $Q \max$ with aging $(8,100,224)$. The Qmax was $19 \%$ lower in group $40,26 \%$ lower in group 50 and $34 \%$ lower in group 60 compared to group 20. In addition, Qmax was $19 \%$ lower in group 60 compared to group 40. Further, at $170 \mathrm{bpm} Q$ was $17 \%$ lower in group 60 compared to group 20 (refer to Figures 6 and 7 and Table 2). These results are in agreement with other researchers who reported a smaller Qmax in older compared to younger ET individuals $(117,122,205,222)$. For instance, Rivera et al. (222) used the acetylene rebreathe method to measure $Q$ and observed a 28\% lower Qmax in older ET males (66 $18 \mathrm{yr}$ ) compared to younger ET males (32 $\pm 5 \mathrm{yr}$ ). Similarly, Ogawa et al. (205) who also used the acetylene rebreathe method, 
observed a 25\% lower Qmax in older ET males (63 $\pm 4 \mathrm{yr}$ ) compared to younger ET males $(28 \pm 3 \mathrm{yr})$. The results of these two investigations are similar to the $26 \%$ lower Qmax observed in group 50 ( $51 \pm 1 \mathrm{yr}$ ) compared to group 20 ( $22 \pm 1 \mathrm{yr}$ ). In addition, Ogawa et al. (205) observed a $22 \%$ lower Qmax in older ET females ( $57 \pm 3 \mathrm{yr}$ ) compared to younger ET females (26 $\pm 3 \mathrm{yr}$ ), which is similar to the $19 \%$ lower Qmax observed in group 60 (60 $\pm 1 \mathrm{yr}$ ) compared to group $40(43 \pm 1 \mathrm{yr})$ and in group 40 compared to group 20 ( $22 \pm 1 \mathrm{yr})$. According to the Fick principle, the mechanisms responsible for the age-related decline in Qmax are decreases in HRmax and/or SVmax.

i) Age-related decline in HRmax

It has been well documented that HRmax declines progressively with advancing age regardless of gender or training status $(6,22,54,60,65,66,83,102,117,122,130,132$, $180,205,214,222,226)$. Maximal HR was $13 \%$ lower in group 50 and $18 \%$ lower in group 60 compared to group 20. In addition, HRmax was $8 \%$ lower in group 50 and $14 \%$ lower in group 60 compared to group 40 (refer to Table 2). However, there were no differences in the HRmax of groups 20 and 40 or of groups 50 and 60 . These results are in agreement with other investigators who have shown that a smaller $Q \max$ is accompanied by a reduced HRmax in older ET males and females compared to younger ET males and females $(117,122$. 205, 222). For instance, Rivera et al (222) observed a $15 \%$ lower HRmax in older ET males ( $66 \pm 8 \mathrm{yr})$ compared to younger ET males ( $32 \pm 5 \mathrm{yr}$ ). Similarly, Heath et al. (122) observed a $14 \%$ lower HRmax in older ET males ( $59 \pm 6 \mathrm{yr})$ compared to younger ET males (18-27 yr) and Hagberg et al. (117) observed a $12 \%$ lower HRmax in older ET males (56 $\pm 5 \mathrm{yr}$ ) 
compared to younger ET males $(25 \pm 3 \mathrm{yr})$. The results of these three investigations are similar to the $14 \%$ lower HRmax observed in group 60 (60 \pm 1 yr) compared to group 40 (43 $\pm 1 \mathrm{yr}$ ) and the $13 \%$ lower HRmax found in group 50 (51 $\pm 1 \mathrm{yr})$ compared to group 20 (22 $\pm 1 \mathrm{yr}$ ). In contrast, Ogawa et al (205) observed only a 7\% lower HRmax in older ET males (63 $\pm 4 \mathrm{yr}$ ) compared to younger ET males ( $28 \pm 3 \mathrm{y}$ ), and an $8 \%$ lower HRmax in older ET females ( $57 \pm 3 \mathrm{yr}$ ) compared to younger ET females ( $26 \pm 3 \mathrm{yr})$. These results are similar to the $8 \%$ lower HRmax observed in group 50 ( $51 \pm 1 \mathrm{yr})$ compared to group $40(43 \pm 1 \mathrm{yr})$. Age-related changes in SVmax will be discussed in the next section of this chapter.

ii) Q-VQ ${ }_{2}$ relationship

The $\mathrm{Q}-\mathrm{VO}_{2}$ relationship was calculated from 3 data points for each subject $(58 \pm 0.8$, $72 \pm 0.2$ and $100 \%$ of $\mathrm{VO}_{2} \max$ ). The slopes for all four age groups were similar, while the intercepts declined progressively as a function of age (Group $20=5.121 \cdot \mathrm{min}^{-1}, 4.281 \cdot \mathrm{min}^{-1}$; Group $40=5.401 \cdot \mathrm{min}^{-1}, 2.331 \cdot \mathrm{min}^{-1}$; Group $50=5.301 \cdot \mathrm{min}^{-1}, 2.46 \mathrm{l} \cdot \mathrm{min}^{-1}$ and Group $60=$ $\left.5.61 \mathrm{l} \cdot \mathrm{min}^{-1}, 1.78 \mathrm{l} \cdot \mathrm{min}^{-1}\right)$. The $\mathrm{QVO}_{2}$ slopes are similar to the $5-6 \mathrm{l} \cdot \mathrm{min}^{-1}$ slopes reported for younger and older males (275) and the $4.6 \mathrm{l} \cdot \mathrm{min}^{-1}$ slope reported for females (66 $\pm 6 \mathrm{yr}$ ) (188). The $4.28 \mathrm{l} \cdot \mathrm{min}^{-1} \mathrm{Q}-\mathrm{VO}_{2}$ intercept in group 20 is similar to the $4.11 \cdot \mathrm{mit}$ intercept reported for younger males (77). The $2.461 \cdot \mathrm{min}^{-1} \mathrm{Q}-\mathrm{VO}_{2}$ intercept in group 50 is similar to the $2.9 \mathrm{l} \cdot \mathrm{min}^{-1}$ intercept reported for older males and females $(\sim 66 \mathrm{yr})(188)$.

\section{Gender Differences in Qmax}

Higher Qmax values have been observed in ET males compared to ET females, regardless of age $(7,205)$. For instance, Ogawa et al. (205) reported that in younger ET 
females, Qmax $\left(1 \cdot \min ^{-1}\right)$ was $33 \%$ lower than age-matched ET males. In agreement, the Qmax of Group 20 was $22 \%$ bwer than the age-matched ET males from the Warburton et al. (289) investigation $\left(24.1 \pm 0.9\right.$ vs $\left.30.0 \pm 0.61 \cdot \mathrm{min}^{-1}\right)$. Mechanisms responsible for the lower Qmax observed in ET females include gender differences in body mass and body composition, as well as differences in SVmax.

i) Gender differences in body mass and body composition

When Ogawa et al. (205) expressed Qmax relative to body mass, the 33\% lower Qmax in the ET females was reduced to $17 \%$. Similarly, when Qmax was expressed relative to body mass, the $22 \%$ lower Qmax in group 20 compared to the ET males was reduced to $10 \%\left(400.8 \pm 18.4 \mathrm{vs} 443.4 \pm 12.7 \mathrm{ml} \cdot \mathrm{kg}^{-1} \cdot \mathrm{min}^{-1}\right)$.

ii) Gender differences in HRmax

Since HRmax does not appear to differ significantly between genders $(5,205,318)$, the smaller Qmax in ET females is due to a smaller $S V \max (7,205)$. In agreement, no difference was observed in the HRmax of group 20 compared to the age-matched ET males in the Warburton et al. investigation (289) (192 \pm 1 vs $194 \pm 1 \mathrm{bpm})$, whereas the ET females had a lower SVmax than the ET males $(125.4 \pm 4.7 \mathrm{vs} 159.6 \pm 4.0 \mathrm{ml})$.

In addition, although investigators have shown that females compensate for their lower $[\mathrm{Hb}]$, during submaximal exercise, with larger increases in $H R$ and $Q$ compared to males $(7,8,91)$, the results of the present investigation revealed no gender differences in $Q$ at each target HR during submaximal exercise. The latter is in agreement with Zwiren et al. (321) and McKelvaney et al. (188) who reported no gender differences in the $\mathrm{Q}-\mathrm{VO}_{2}$ slope 
in males and females, suggesting the change in $\mathrm{Q}$ for a given $\mathrm{VO}_{2}$ during exercise is not gender related.

\section{Stroke Volume in ET Individuals and the Relationship to Age and Gender}

In ET individuals, a larger SVmax (ml) has been observed in both younger and older ET males $(7,11,67,68,82,105,117,122,152,205,222,234,245,308,309)$, as well as in both younger and older ET females (205), compared to age and gender-matched NA individuals. Endurance training related increases in SV may be explained by an increase in LVEDV and/or by a decrease in LVESV, since SV = LVEDV - LVESV. An increased LVEDV is attributed to increased cardiac dimensions $(104,131,198,209,279)$, improved compliance of the LV cavity (312), and/or enhanced preload, resulting from enhanced venous return $(145,227,309)$ and elevated BV $(35,220)$. A decreased LVESV is attributed to enhanced myocardial contractility $(3,260)$ and decreased afterload $(5,145,227,309)$.

However, controversy exists regarding the mechanisms which are primarily responsible for the increased exercise SV observed in ET compared to NA individuals. It was previously believed that the Frank-Starling mechanism has its greatest influence at low to moderate work rates while myocardial contractility has its greatest influence at higher work rates $(7,104,108,123,211,268)$. These investigators and others observed that $S V$ increases until it reaches a plateau at exercise intensities between $40-60 \%$ of $\mathrm{VO}_{2} \max (7,104,108,123$, 211, 268). Researchers believed that at $H R$ above $170 \mathrm{bpm}$, a decreased DT would limit ventricular filling and thus EDV $(104,123,211)$, resulting in a plateau in SV. Therefore, any further increases in $\mathrm{Q}$ beyond $40-60 \%$ of $\mathrm{VO}_{2} \max$ would be due to increases in $\mathrm{HR}$ and 
enhanced myocardial contractility $(104,108,123,211)$.

In contrast, however, recent investigators observed that ET males have the ability to use the Frank-Starling mechanism effectively up to $\mathrm{VO}_{2} \max (46,105,128,152,165,220$, 230). For example, Rerych et al (220), found that endurance training resulted in an enhanced EDV, while HR and EF were maintained. The authors postulated that training resulted in an enhanced capacity to use the Frank-Starling mechanism since an increased EDV was the primary mechanism responsible for the increased Qmax. In agreement, Crawford et al (46), observed an enhanced EDV during the later stages of exercise in competitive male marathon runners compared to noncompetitive male runners. These authors postulated that ET males utilize the Frank-Starling mechanism to a greater extent during strenuous exercise than NA males.

Some investigators used the acetylene rebreathe method to measure $Q(105, .152,222$, 289). Gledhill et al. (105) and Krip et al. (152) examined cardiac function in ET compared to NA males, during incremental cycle ergometer exercise to maximum. Although they observed a plateau, in SV at $\sim 40 \% \mathrm{VO}_{2} \mathrm{max}$ in the NA males, the $\mathrm{SV}$ of the ET males increased progressively, throughout incremental exercise to maximum without a plateau. Similarly, Rivera et al. (222) observed that SV did not reach a plateau, but continued to increase up to maximum in 5 out of 11 ET male masters runners $(66 \pm 8 \mathrm{yr})$ who were examined. Providing further support for this finding, Warburton et al. (289) observed a progressive increase in SV up to maximum, without a plateau in their nine elite male cyclists. In agreement, the SV of group 20 in the present study continued to increase significantly up 
to maximum exercise without reaching a plateau. Although it cannot be determined directly from the data in the investigation by Warburton et al. (289) or that of the present investigation, whether the progressive increase in SV to maximum observed in group 20 resulted from an increase in EDV, a decrease in ESV or a combination of the two factors, it appears that both males and females may have the ability to use the Frank-Starling mechanism throughout incremental exercise to maximum. Evidence to support this comes from the responses of PEP (an index of myocardial contractility), which reached a plateau at $130 \mathrm{bpm}$ in the ET females (Figure 13), and $150 \mathrm{bpm}$ in the ET males from the Warburton et al (289) investigation. This suggests that contractility does not progressively increase throughout incremental exercise to maximum, and supports the recent research indicating that ET individuals may increase their SV during the later stages of vigorous exercise through the use of the Frank-Starling mechanism. In order to increase SV via the Frank-Starling mechanism, ET individuals must increase their EDV at high HR despite a significantly shortened DT.

\section{Age-Related Changes in SVmax}

Controversy exists regarding the age-related changes in SVmax. While some investigators have shown an age-related reduction in $S V \max (110,139,205,222,267)$, others have observed no change in SVmax with aging $(81,117,122,318)$, while others have shown an increase in SVmax with age $(8,100,224)$. Although not statistically significant, a trend towards a lower SVmax was observed as a function of age in the ET females from the present investigation (Figures 8 and 9 and Table 2). It is possible that if the $n$ were increased, these results would have been significant. Furthermore, in contrast to group 20, groups 40 , 
50 and 60, did not display a progressive increase in SV throughout incremental exercise to maximum. As discussed earlier, it cannot be determined directly from the data whether the plateau in SV or the trend towards an age-related decline in SVmax was due to a decrease in LVEDV and/or an increase in LVESV.

i) LVESY

Researchers have observed that in contrast to younger individuals there is little or no reduction in LVESV at high exercise intensities in older individuals, resulting in a smaller increase in EF from rest to peak exercise $(125,215,224,318)$. Age-related decreases in contractile reserve, resulting from decreased $\beta$-AR responsiveness of cardiac tissue $(156,215)$ and decreased ventricular compliance (201) have been implicated as contributing factors for this age-related change. Since there were no group differences in PEPmax (refer to Figure 13 or Table 2), this suggests that the contractile reserve was not reduced as a function of age in the ET females. This is in agreement with Seals et al. (245) who observed enhanced myocardial contractility in older ET males ( $64 \pm 2 \mathrm{yr}$ ) compared to age-matched sedentary males and Ehsani et al. (66) who observed that in response to $1 \mathrm{yr}$ of endurance training older males ( $64 \pm 3 \mathrm{yr}$ ) displayed enhanced myocardial contractility.

Additionally, increased afterload (evident in enhanced BP responses), resulting from increased vascular resistance and aortic impedance (316), has also been implicated as a contributing factor to the age-related decline in $\mathrm{EF}$ during exercise. Investigators have observed age-related increases in peak exercise DBP and MAP in healthy females 50-69 yr $(51,130,181,190)$. In ET females, Ogawa et al. (205) observed significantly higher SBP, 
DBP and TPR responses throughout treadmill exercise to maximum, in older ET females (57 $\pm 3 \mathrm{yr}$ ) compared to younger ET females ( $26 \pm 3 \mathrm{yr}$.). Similarly, Martin et al. (181) observed that in comparison to younger ET females $(27 \pm 3 \mathrm{yr})$, older ET females $(65 \pm 3 \mathrm{yr})$ had a significantly higher SBPmax and MAPmax, with no difference in DBPmax. However, these age-related differences were not observed in females treated with ERT. Since only one of the females from the present investigation was using HRT, any effect estrogen may have on the BP responses during maximal exercise cannot be determined.

The DBPmax was $24 \%$ higher in group 60 compared to group 20 (refer to Table 2). The higher DBPmax in group 60 was reflected in a $13 \%$ higher MAPmax in this group compared to group 20. Further, MAPmax was $13 \%$ higher in group 60 compared to group 40 and $10 \%$ higher in group 60 compared to group 40 (refer to Figure 21). One mechanism responsible for the higher MAPmax in these groups is the higher TPRmax. The TPRmax was $71 \%$ higher in group 60 compared to group $20,41 \%$ higher in group 50 compared to group 20 and $39 \%$ higher in group 60 compared to group 40 (refer to Table 2). It has been postulated that age-related increases in MAP and DBP reflect an increase in systemic vascular resistance, resulting from a decrease in peripheral vasodilatory capacity and an increase in arterial stiffness $(102,284)$. Thus, one mechanism responsible for the plateau observed in the SV of groups 50 and 60 , may be the greater TPRmax observed in these two groups compared to group 20, which in turn results in an increased afterload and thus an impedance to systolic emptying. 
ii) LVEDY

It has been suggested that the mechanism through which older males and females augment SV during exercise, shifts from a dependence on catecholamine mediated increases in HR and inotropy to a greater use of the Frank-Starling mechanism. It was postulated that compensation for decreased $\beta-A R$ responsiveness of cardiac tissue and/or increased afterload, is brought about via an elevation in EDV, resulting from LV dilation $(81,179,224,241$, 318). Although LVEDV could not be measured, the fact that there were no group differences in BV (refer to Figure 3 and Table 2), suggests that preload and thus, EDV may have been maintained as a function of age in the ET females.

\section{Gender Differences in SVmax}

Higher SVmax values have been observed in ET males compared to females, regardless of age $(7,205)$. For instance, Ogawa et al. (205) reported that in younger ET females, SVmax was $34 \%$ lower than in age-matched ET males. In agreement, the SVmax of group 20 was $21 \%$ lower than the age-matched ET males from the Warburton et al. investigation (289) $(125.4 \pm 4.7 \mathrm{vs} 159.6 \pm 4.0 \mathrm{ml})$. Mechanisms responsible for the lower SVmax observed in ET females include differences in body mass and body composition, differences in heart size, as well as differences in the mechanisms responsible for augmenting SVmax (preload, myocardial contractility and afterload).

i) Gender differences in body mass and heart volume

Stroke volume is determined to a large extent by body size and thus, as a result of a smaller body mass, an ET female will have a smaller SV. For instance, when Ogawa et al. 
(205) expressed SVmax relative to body mass, the 34\% lower SVmax in the ET females compared to ET males was reduced to $18 \%$. Similarly, when the $21 \%$ lower SVmax of group 20 was expressed relative to body mass, SVmax was only $8 \%$ lower in this group compared to the ET males of the Warburton et al. (289) investigation (2.09 \pm 0.09 vs $2.28 \pm 0.07 \mathrm{ml} \cdot \mathrm{kg}^{-}$ $\left.{ }^{1} \cdot \mathrm{min}^{-1}\right)$. In addition, the literature has shown that regardless of training status, because of a smaller body mass, females will have a smaller heart (volume) in both absolute and relative terms, compared to age-matched males $(109,200)$. A smaller heart in females may reduce LV filling capacity and thus, SVmax (7). Since cardiac volumes were not measured in the present investigation, it cannot be determined whether a smaller heart volume contributed to the gender-related differences in SVmax.

ii) Gender differences in the augmentation of SVmax

The majority of investigators who examined the mechanisms responsible for augmenting SVmax have shown that independent of body position or age, both ET and NA females rely to a greater extent on the Frank-Starling mechanism (and thus, preload) to augment $S V \max$, while males rely on changes in $\mathrm{EF}$ (and thus contractility) $(1,72,81,118$, $125,200,229,231,319)$. However, the overall picture is controversial (refer to Literature Review). As discussed earlier, it cannot be directly determined from the data in this investigation, or the Warburton et al. (289) study whether the lower SVmax of the ET females compared to the ET males is due to a decreased LVEDV (and thus preload), an increased LVESV (and thus afterload or decreased contractility) or a combination of the factors. Since an augmented BV is associated with an increased prelaod and thus LVEDV 
and SV $(128,142)$ the lower absolute BV of group 20 compared to the ET males (5039 \pm 164 vs $6648 \pm 310 \mathrm{ml}$ ), may contribute to the ET females lower SVmax. Further, since PEP (a measure of contractility) plateaued at $\sim 50 \%$ of $\mathrm{VO}_{2} \max$ in both genders, this suggests that contractility is not a limiting factor for the lower SVmax observed in the ET females. However, since the ET females had a higher TPRmax than the ET males $(4.37 \pm 0.20$ vs 3.54 $\pm 3.54 \mathrm{mmHg} \cdot\left(\cdot \mathrm{min}^{-1}\right)$, this would result in greater afterload, and thus a larger LVESV in the ET females compared to the ET males. Therefore, it appears that the mechanisms responsible for the smaller SVmax observed in the ET females compared to the ET males, are likely the smaller BV of the ET females, which in turn results in a decreased preload and LVEDV, as well as the higher TPRmax of the ET females, which in turn results in an increased afterload and LVESV.

\section{Arteriât-Mixes Venous $\mathrm{Q}_{2}$ Difference in ET Individuals and the Relationship to Age and Gender}

A larger $\mathrm{a}-\mathrm{wDO}_{2} \max$, has been observed in both younger and older ET males $(11,205$, 222,240 ), as well as in both younger and older ET females (205), compared to age and gender-matched NA individuals. During maximal exercise, oxygen is consumed primarily by the mitochondria of the active skeletal muscle fibres. Mechanisms responsible for the larger a- $\overline{\mathrm{vDO}}{ }_{2} \mathrm{max}$ in ET compared to NA individuals are: increases in mitochondrial density, increases in oxidative capacity of the active skeletal muscles via increases in oxidative enzyme activity and capillary density, which in turn result in increases in muscle oxygen extraction capacity $(30,31,225,234)$. 


\section{Age-Related Changes in a-vidO $\max$}

Ogawa et al (205) showed that $\mathrm{a}$ - $\mathrm{iDO} \mathrm{D}_{2} \max$ does not differ between older $(63 \pm 4 \mathrm{yr})$ and younger ET males ( $28 \pm 3 \mathrm{yr})$, or older $(57 \pm 3 \mathrm{yr})$ and younger ET females $(26 \pm 3 \mathrm{yr})$.

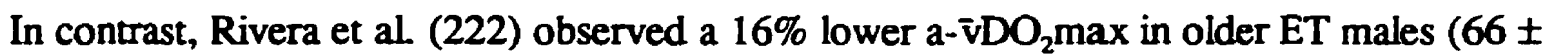
$8 \mathrm{yr}$ ) compared to younger ET males ( $32 \pm 5 \mathrm{yr}$ ). In agreement with Ogawa et al. (205), no age-related differences existed in the a- $\mathrm{vDO}_{2} \max$ of the ET females (refer to Figures 10 and 11 and Table 2). It was postulated that ET individuals maintain $a-\bar{v} \mathrm{vO}_{2} \max$ with age through a maintenance in skeletal muscle oxidative capacity and capillary density $(30,225)$. Additionally, it is interesting to note that investigators examining older sedentary males (63 $\pm 3 \mathrm{yr}$ ) and females (63 $\pm 4 \mathrm{yr}$ ) who became endurance trained over a period of 9-12 mo (4-5 $\mathrm{d} \cdot \mathrm{wk}^{-1}, 45 \min 1 \mathrm{hr} \cdot \mathrm{d}^{1}, 60-85 \%$ of $\left.\mathrm{VO}_{2} \max \right)(257,258)$, revealed that the entire traininginduced elevation in $\mathrm{VO}_{2} \max$ was due to increases in a- $-\mathrm{vO}_{2} \max$ in the females, while cnly $34 \%$ of the elevation in $\mathrm{VO}_{2} \max$ in the males was due to increases in $\mathrm{a}-\overline{\mathrm{vDO}} \mathrm{D}_{2} \max (257,258)$. Thus, it appears that ET females may be able to maintain their $\mathrm{a}-\mathrm{v} \mathrm{vDO}_{2} \max$ with age and that older sedentary females may possess a greater capacity for augmenting a- $\overline{\mathrm{v}} \mathrm{DO}_{2} \max$ in response to endurance training, compared to older sedentary males. This is an area which requires further investigation in females. In summary, the age-related decrease in $\mathrm{VO}_{2} \max$ in ET females was due mainly to a decline in HRmax, with a trend towards a decline in SVmax, and no change in a- $\overline{\mathrm{vDO}} \mathrm{O}_{2} \max$.

\section{Gender Differences in a-ī $\mathrm{DO}_{2} \max$}

Investigators have observed similar values for $\mathrm{a}-\mathrm{wDO}_{2} \max \left(\mathrm{ml} \mathrm{O}_{2} \cdot 100 \mathrm{ml}^{-1}\right)$ in younger 
ET males and females $(194,205,321)$. In agreement, no differences were observed in the a$\overline{\mathrm{vDO}}{ }_{2} \max$ of group 20 compared to the age-matched ET males from the investigation by Warburton et al. (289) $\left(16.0 \pm 0.5 \mathrm{vs} 15.6 \pm 0.5 \mathrm{ml} \mathrm{O}_{2} \cdot 100 \mathrm{ml}^{-1}\right)$. However it is of interest to note that, when the differences in a- $\bar{v} \mathrm{OO}_{2} \max$ are expressed relative to body mass, group 20 had a $19 \%$ higher $a-\bar{v} \mathrm{OO}_{2} \max$ than the ET males $\left(2.66 \pm 0.09\right.$ vs $2.24 \pm 0.06 \mathrm{ml} \mathrm{O}{ }_{2} \cdot \mathrm{kg}^{-1}$. $\mathrm{dl}^{-1}$ ). In agreement, Ogawa et al. (205) who also used the acetylene rebreathe method, observed a $19 \%$ larger relative $\mathrm{a}-\mathrm{ZDO}_{2}$ max in younger ET females compared to age-matched ET males. This difference seemed to be maintained with aging, such that older ET females, from the same investigation, had a $9 \%$ higher relative a- $\mathrm{vDO}_{2}$ max than age-matched ET males, although no gender differences existed for absolute $a-\bar{v} D O_{2} \max (205)$. Similarly Spina et al. (257), observed a $12 \%$ larger relative $a-\bar{v} D_{2} \max$ in older sedentary females compared to age-matched sedentary males, with this difference increasing to a $24 \%$ greater-relative a$\mathrm{vDO}_{2} \mathrm{max}$ in the older females compared to the males after an endurance training program. Since neither Ogawa et al. (205) nor Spina et al. (257) analyzed or discussed this data, no reasons were given for this gender-related difference.

Although, it has been postulated that the potential for oxidative metabolism is higher in females than in males. For instance, females show greater increases in the activity of phosphofructokinase, while males show greater increases in the activity of succinate dehydrogenase and citrate synthase in response to endurance training $(30,204,253)$. The ratio between the activity of the two enzymes shows that the potential for oxidative metabolism is greater in females than in males (151). Additionally, it has been postulated that 
since estrogen enhances the concentration of growth hormone (90), which in turn increases lypolysis and reduces glycogenolysis via its supressing action on epinephrine, estrogen may contribute to the greater potential for oxidative metabolism in females. Further, although no investigators to date have examined this (to the authors knowledge), it is conceivable that in compensation for their lower $Q$, ET females may be able to enhance their skeletal muscle capillarization (via increases in capillary density) to a greater extent than ET males, thus maintaining capillary mean transit time, and making possible a larger $\mathrm{O}_{2}$ extraction, and a$\overline{\mathrm{vDO}}{ }_{2} \max \left(\mathrm{ml} \mathrm{O}_{2} \cdot \mathrm{kg}^{-1} \cdot \mathrm{dI}^{-1}\right)$.

In summary, the lower $\mathrm{VO}_{2}$ max observed in group 20 , compared to the ET males from the Warburton et al. (289) investigation, was due to a lower Qmax, which resulted from a lower SVmax. No gender-related differences were observed for $\mathrm{HRmax}$ or for a- $\mathrm{vDO} \mathrm{O}_{2} \max$. Although group 20 showed a similar progressive increase in SV throughout incremental exercise to maximum, the lower SVmax of group 20 was due to a lower BV in this group, compared to the ET males from the Warburton et al. (289) investigation. However, when expressed relative to body mass, although $\mathrm{VO}_{2} \max$ was still larger in the ET males, no gender-related differences were evident for $\mathrm{Qmax}$ or for $\mathrm{SV \operatorname {max }}$, while a- $\mathrm{v} \mathrm{DO}_{2} \max$ was larger in the ET females. Further research is needed to examine the mechanism responsible for the gender-related difference in relative $\mathrm{a}-\mathrm{vDO}_{2} \mathrm{max}$, in both NA and ET individuals.

\section{Cardiac Time Intervals in ET Individuals and the Relationship to Age and Gender}

The CTI of interest are LVET, PEP and DT. The LVET and PEP are inversely and linearly related to HR (293). Thus, to compare differences in CTI in individuals at rest, 
correction must be made for variations in HR (292). The LVET varies directly with an enhanced ventricular preload, SV and afterload and inversely with inotropy $(121,177,292)$. The LVET displayed the typical pattern observed previously in males during incremental exercise to maximum $(44,152,289)$, such that it decreased progressively with increasing HR (refer to Figure 12). The PEP varies inversely with ventricular filling, SV and inotropy (177, 195, 320). The PEP also displayed the typical pattern observed previously in ET males (44, 152,289 ). For instance while PEP reached a plateau at $130 \mathrm{bpm}$ in the ET females (Figure 13), it reached a plateau at $150 \mathrm{bpm}$ in the ET males from the Warburton et al. (289) study. The DT is also inversely related to HR, although non-linearly, and is primarily influenced by changes in LVET and PEP. For instance, a prolonged LVET or PEP will result in a shortened DT, while a shortened LVET or PEP will result in a prolonged DT. The DT (refer to Figure 14) also displayed the typical pattern observed in previcus investigations of males $(44,152,289)$.

Endurance trained compared to NA individuals generally have a prolonged LVET at rest and during exercise $(75,76,105,152,154,195,311)$, due to their larger $B V$, and thus, SV (177). It has been postulated that a longer time is required for the ejection of a larger volume of blood, per beat. However, training related changes in PEP are controversial, with some investigators observing no differences in PEP (44), and with others observing a prolonged PEP in ET compared to NA males $(152,320)$. Since PEP is primarily affected by inotropic stimulation (291) a prolonged PEP may result from a decrease in sympathetic activation. Cox et al. (44) postulated that since their ET and NA males were matched for HR 
at all levels of exercise, no difference in adrenergic status would be expected to occur between groups, and thus similar PEP values should have resulted.

As a result of a lengthened LVET in ET individuals, the period of systole constitutes a larger portion of the cardiac cycle, thus, shortening DT $(76,105,152,154)$. Although a decreased DT in ET individuals seems to be counterproductive for increasing LVEDV and thus, SV during exercise, investigators have observed enhanced DFR in ET compared to NA males $(105,152)$ which would thus compensate for the shortened DT. In contrast, other investigators observed a prolonged resting DT in ET compared to NA males $(75,76,152$, 167) while some others have seen no difference in resting DT (79). Levy et al. (167) postulated that the prolonged resting DT observed in response to 6 mo of endurance training in younger ( $\sim 28 \mathrm{yr})$ and older males $(\sim 68 \mathrm{yr})$ was due to the HR lowering effect of endurance training, which resulted in a lengthenirg of the entire cardiac cycle and thus, DT. Hence, some of the variability in results may partly be due to a failure to control for the effects of resting HR on DT, since unlike the other resting CTI (LVET, PEP) no correction factor has been developed for DT.

\section{Age-Related Changes in CTI}

To date there have been no investigations of CTI which compare ET females across age groups during incremental exercise to maximum. Thus, there are no population-specific data available with which to compare the present results. As a consequence of aging, at rest and even more so during exercise, NA individuals have a prolonged LVET and PEP, at the expense of DT $(251,286,301)$. 


\section{i) LVET}

Willems et al. examined a combined group of younger individuals (302) and elderly individuals (301) and observed a small but significant influence of age on resting LVET, independent of HR and BP. Similarly, in an examination of children, adolescents and adults, Vavra et al (286) observed a slight lengthening of LVET (20-30 msec), especially with exercise in older males (45-55 yr) compared to both boys (11-14 yr) and younger males and females (20-30 yr). Similarly, after correction was made for resting HR (292), although not statistically significant, resting LVET was slightly prolonged as a function of age in the ET females (refer to Figure 12). Possible reasons for the age-related lengthening of resting LVET are: a decline in myocardial contractility, due to a decrease in $\beta$-AR responsiveness, and/or an increase in afterload to the LV due to an increase in aortic impedance or an increase in peripheral resistance. Since PEP was not related to age in the ET females at any of the target HR, this suggests that myocardial contractility may have been maintained with aging. In addition, although resting TPR was not significantly different between groups, it appeared to increase slightly as a function of age (refer to Figures 23 and 24), suggesting an increased afterload. However, no significant age-related differences existed for LVET at any of the matched HR during exercise. This is in agreement with the direct relationship which exists between LVET and preload (BV) $(75,76,169,195)$. Since BV was maintained as a function of age in the ET females, LVET should have also been maintained (refer to Table 2 and Figure 3). Further, there were no group differences in the percentage of the cardiac cycle represented by LVET at $150 \mathrm{bpm}$ (group $20=51 \%$, group $40=52 \%$, group $50=55 \%$ and 
group $60=55 \%$ ).

\section{ii) $\quad$ PEP}

Wanderman et al. (288), observed a distinct tendency towards lengthening of PEP with increasing age, independent of HR. Similarly, Shaw et al. (251) examined males (20-89 yr) and observed a prolonged PEP, of $4 \mathrm{msec} \cdot$ decade $^{-1}$ from 30 to $60 \mathrm{yr}$, and a shortening, thereafter. In agreement, Montoye et al (197) observed a prolonged PEP of $4 \mathrm{msec} \cdot$ decade $^{-1}$ from 30 to $70 \mathrm{yr}$, with a shortening thereafter. In contrast to these investigations, the ET females in the present investigation did not display any significant age-related changes in PEP at rest or at any of the matched HR during exercise (refer to Figure 13 and Table 2). This suggests that myocardial contractility may be maintained as a function of age in ET females. iii) DT

A prolonged PEP and LVET at the same HR, correspondingly reduce DT. For instance, Vavra et al (286) observed a shortening of DT, such that the boys' DT represented $35 \%$ of the cardiac cycle, while the older males' DT represented only $25 \%$ of the cardiac cycle. In contrast, there were no age-related differences in DT at rest or at any of the matched HR during exercise (refer to Figure 14). Further, no group differences existed in the percentage of the cardiac cycle represented by DT at $150 \mathrm{bpm}$ (group $20=37 \%$, group 40 $=40 \%$, group $50=38 \%$ and group $60=38 \%$ ) .

Additionally, as the cardiac cycles shorten with increasing HR, DT shortens much faster than either LVET or PEP. Thus, with progressively increasing HR, diastolic filling requires progressively less time to accomplish the same SV as does systolic emptying. Hence, 
at HRmax, in group 20; LVET required 55\% of the cardiac cycle, while DT occupied only $35 \%$, in group 40; LVET required $56 \%$ of the cardiac cycle, while DT occupied only $38 \%$, in group 50; LVET required 55\% of the cardiac cycle, while DT occupied only $37 \%$, and in group 60; LVET required 53\% of the cardiac cycle, while DT occupied only 39\% (Figures 12 and 14). Therefore, this provides support for the finding that diastolic function (rather than systolic function) is the differentiating factor of an enhanced cardiac function $(70,105$, $152,186,289)$.

In summary, in contrast to other investigations of NA individuals, no significant agerelated differences existed for the CTI of the ET females. However, since the individuals in the other investigations were NA, direct comparisons to the present ET population of females may not be appropriate.

\section{Gender Differences in-CTI}

\section{i) LVET}

Independent of HR, LVET is significantly longer in females compared to males (74, 171, 294, 301). For instance, Falsetti et al. (74) examined female and male competitive cyclists (19-31 yr), with $\mathrm{VO}_{2} \max$ values of 42 and $61 \mathrm{ml} \cdot \mathrm{kg}^{-1} \cdot \mathrm{min}^{-1}$, respectively. After correction was made for resting HR, they observed LVET values of 410 and $396 \mathrm{msec}$, in the females and males respectively. Lombard et al. (171) suggested that the mechanism responsible for the lengthened LVET in females, is their smaller heart size in proportion to their body size. They hypothesized that if similar amounts of blood were sent to the heart, a females smaller heart would be enlarged more than a males heart by the entering blood, and 
thus would need more time to eject the blood (171). However, since the BV of the ET females was smaller than the BV the ET males, this hypothesis may not be applicable to the present investigation.

After correction for differences in HR, the ET females required less time for LVET at rest than the ET males from the Warburton et al. (289) investigation (359 \pm 2 vs $375 \pm 4$ msec). Furthermore, the percentage of the cardiac cycle represented by the resting LVET was slightly smaller in the ET females compared to the ET males (33\% vs $39 \%$, respectively). Thus, in agreement with the direct relationship which exists between LVET and preload (BV) $(75,76,169,195)$, the larger BV observed in the ET males compared to the ET females (6648 \pm 310 vs $5039 \pm 164 \mathrm{ml})$, resulted in a prolonged resting LVET in the ET males. Further, it is possible that the phenomena "crossing of the curves" (171), may be occuring here, such that at very slow HR the LVET of a female becomes shorter than that of a male. Although the gender difference in LVET disappeared throughout submaximal exercise, LVETmax was prolonged in the ET females compared to the ET males (175 \pm 5 vs $163 \pm 2$ msec). Further, the percentage of the cardiac cycle represented by LVETmax was slightly longer in the ET females compared to the ET males (55\% vs 53\%, respectively). However, the primary mechanisms responsible for this difference was the slightly lower HRmax of the ET females compared to the ET males (192 \pm 1 vs $194 \pm 1 \mathrm{bpm}$ ). If the ET males and females had been compared at matched HR, LVETmax would not have differed between genders. 


\section{ii) $\quad$ PEP}

Falsetti et al. (74), who examined female and male competitive cyclists (19-31 yr), observed no differences in the resting PEP after correction was made for resting HR (118 and $122 \mathrm{msec}$, respectively). In contrast, group 20 had a longer resting PEP than the ET males from the Warburton et al. (289) investigation after correction for differences in resting HR (212 \pm 12 vs $168 \pm 5 \mathrm{msec}$ ), as well, the percentage of the cardiac cycle represented by resting PEP was slightly longer in the ET females compared to the ET males (20\% vs $18 \%$, respectively). Since PEP varies inversely with ventricular filling, SV and inotropy $(177,195$, 291, 320) a prolonged PEP may result from the lower BV and thus ventricular filling in the ET females compared to the ET males, or from lower sympathetic activity and thus, inotropy in the ET females compared to the ET males. However, there were no other gender differences in PEP throughout incremental exercise to maximum.

iii) DT

Although DT has not previously been measured in females, a prolonged LVET in NA females compared to NA males, would be expected to result in a subsequent shortening of DT in the females compared to the males. In contrast, since group 20 required less time for resting LVET, they in turn, required more time for resting DT than the ET males (503 \pm 33 vs $411 \pm 25 \mathrm{msec}$, respectively). Further, the percentage of the cardiac cycle represented by the resting DT was slightly greater in the ET females compared to the ET males (47\% vs $43 \%$, respectively). However, some of the gender difference in resting DT may be due to a failure to control for the effects of resting HR on DT, since unlike the other resting CTI 
(LVET, PEP) no correction factor has been developed for DT. Although this difference disappeared throughout submaximal exercise, the ET females also required more time for DTmax than the ET males $(111 \pm 2$ vs $104 \pm 1 \mathrm{msec}$, respectively). Further, the percentage of the cardiac cycle represented by DTmax was slightly longer in the ET females compared to the ET males ( $35 \%$ vs $34 \%$, respectively). However, the primary mechanism responsible for this difference was the slightly slower HRmax of the ET females compared to the ET males (192 \pm 1 vs $194 \pm 1 \mathrm{bpm}$ ). If the ET males and females had been compared at matched HR, DTmax would not have differed between genders.

Thus, it appears that due to the smaller BV of an ET female compared to an ET male, the loading conditions on the heart are different, such that an ET female requires less time for LVET, and more time for both PEP and DT at rest. However, at matched HR throughout submaximal exercise, ET females display similar endurance training-induced adaptations in CTI as observed in ET males.

\section{Blood Volume in ET Individuals and the Relationship to Age and Gender}

Both cross-sectional $(15,59,149,265)$ and longitudinal investigations $(23,37,103$, $113,192,206)$ consistently show an augmented BV in ET compared to NA individuals. Blood volumes in the range of 90 to $104 \mathrm{ml} \cdot \mathrm{kg}^{-1}$ have been reported in younger ET males compared to $\sim 75 \mathrm{ml} \cdot \mathrm{kg}^{-1}$ in age-matched NA males $(15,59,149)$, while BV of $89 \mathrm{ml} \cdot \mathrm{kg}^{-1}$ have been reported for younger and older ET females, compared to $62 \mathrm{ml} \cdot \mathrm{kg}^{-1}$ for age-matched NA females $(23,127,136,149,192,265)$. Thus, cross-sectional investigations reveal that ET individuals have a BV that is $\sim 20-25 \%$ larger than their age-matched NA counterparts ( 15 , 
$59,149)$, while participants in longitudinal investigations experience an increase in BV of only $\sim 7 \%$ (35). This suggests that other factors than endurance exercise may be responsible for the increased BV, such as: initial fitness level, duration of previous exposure to endurance training, or genetics. The later possibility has gained interest lately. It is possible that ET individuals are genetically endowed with an elevated $\mathrm{BV}$, enabling them to be naturally selected into endurance events (152). Endurance trained individuals have a larger BV, primarily due to an expanded PV $(127,149)$ and red cell volume $(\mathrm{RCV})(2,24,192)$.

\section{Age-Related Changes in BV}

Although BV has previously been observed to decrease as a function of age in NA individuals (53), the capacity to increase PV, and thus BV with endurance training is maintained as a function of age (24). For instance, Jones et al. (136) observed similar high BV in ET premenopausal (29 yr) and postmenopausal (62 yr) females ( 85.2 and $84.7 \mathrm{ml} \cdot \mathrm{kg}^{-1}$, respectively). The PV and RCV were also similar between groups. These authors concluded that $\mathrm{BV}$ is maintained with age in ET females. In agreement with the literature, no agerelated differences existed for $\mathrm{BV}$ in $\mathrm{ml}$ or $\mathrm{ml} \cdot \mathrm{kg}^{-1}$ (refer to Figure 3 or Table 2). Similarly, when the eight postmenopausal females (not using HRT) were compared to the eleven premenopausal females (not using OCA) no difference was observed for BV in $\mathrm{ml}$ or $\mathrm{ml} \cdot \mathrm{kg}^{\prime}$.

Stevenson et al. (265) reported no differences in BV, PV or RCV in ET females who were using HRT compared to those who were not. In agreement, Bush et al. (21) examined active female HRT users and non-users $(48-60 \mathrm{yr})$ and concluded that HRT does not influence exercise-induced BV shifts in post-menopausal females. Since there was only one 
HRT user in the twenty-three ET females examined in the present investigation, a comparison could not be made.

However, controversy exists regarding the influence of OCA on PV, with some investigators observing an increase in BV (164), while others showed no change (163). It has been postulated that when haematology examination is conducted during the same phase of the menstrual cycle, (ie. follicular phase), hormonal levels are not significantly different between OCA users and non-users, and thus [Hb], Hct, PV and BV should also not differ. When the three OCA users were compared to the eleven premenopausal females not using OCA, no difference was observed for $\mathrm{BV}$ in $\mathrm{ml}$ or $\mathrm{ml} \cdot \mathrm{kg}^{-1}$. Thus, in agreement with the literature it appears that $\mathrm{BV}$ is maintained as a function of age in ET females.

\section{Gender Differences in BV}

As previously discussed, ET males and females display similar training-induced augmentations in BV, expressed relative to body mass (149). In a more recent study, Mier et al (192), observed that $10 \mathrm{~d}$ of cycle ergometer training in sedentary males $(29 \pm 2 \mathrm{yr})$ and females $(25 \pm 2 \mathrm{yr})$ resulted in a significant increase in BV and SV in both genders, and, although males had a larger absolute BV, when expressed relative to body mass, no gender differences existed. It was postulated that males and females posses similar cardiac reserve capacities for increasing BV as a result of endurance training (192). In the present investigation, group 20 had a $24 \%$ lower absolute BV than the ET males from the Warburton et al. (289) investigation ( $5039 \pm 164$ vs $6648 \pm 310 \mathrm{ml}$ ), and when expressed relative to body mass the ET females had a $12 \%$ smaller BV than the ET mals $(83.6 \pm 1.5$ vs $95.4 \pm 3.4$ 
$\left.\mathrm{ml} \cdot \mathrm{kg}^{-1}\right)$. Thus, although both ET males and females display training-induced elevations in BV, ET females have lower BV values.

\section{Diastolic Filling Rate and LVER in ET Individuals and the Relationship to Age and}

\section{Gender}

Recent research has shown that in contrast to the classical explanation of an enhanced systolic function being the major determinant of an augmented cardiac function, an enhanced diastolic function plays a greater role $(105,186)$. Furthermore, in ET males, an elevated DFR is the primary determinant of an enhanced cardiac function $(70,105,152,186,289)$, while an elevated LVER plays little or no role $(11,71,79,166,279)$. In the present investigation, DFR was calculated as SV/DT and LVER was calculated as SV/LVET. It is important to note that the DT used to calculate DFR includes the isovolumic relaxation period. Since "true" diastolic filling occurs after the opening of the mitral valve, DT may be substantially shorter than that reported in this investigation, and thus the "true" DFR may be even greater than that reported.

\section{Age-Related Changes in DFR and LVER}

Controversy exists regarding the age-related changes in DFR in ET individuals. While some investigators observed no difference in the DFR of older ET males compared to sedentary age-matched males $(82,243)$, others showed an attenuation or reversal of this phenomenon with endurance training $(62,85,167,256)$. For instance, Fleg et al. (82) observed that older ET males (52-76 yr) demonstrated impaired early LV filling with compensatory increases in late atrial filling, compared to younger individuals $(<40 \mathrm{yr})$, and 
reported that this attenuation was similar in magnitude to that observed in sedentary agematched males. Similarly, Schulman et al. (243) examined healthy sedentary and ET males (22-82 yr). The decline in resting and exercise DFR were similar in magnitude in the ET older males and the sedentary older males. Thus, these two investigations suggest that the impairment in early diastolic filling is not secondary to the reduction in aerobic capacity that occurs with aging.

In contrast, Takemoto et al. (270) examined male distance runners $(60 \pm 1 \mathrm{yr})$ and sedentary age-matched males. In comparison to the sedentary males, the ET males had a decreased late peak filling velocity, and an increased E/A peak filling velocity. Forman et al. (85) observed exactly the same results when they examined younger sedentary males (27-32 yr), and older sedentary males (58-68 yr) and masters males (52-66 yr). Furthermore, in response tc 6 mo of endurance training, Levy et al. (167) observed a $14 \%$ increase in the absolute peak DFR at rest and during exercise in previously sedentary younger males (28 yr) and older males ( $68 \mathrm{yr})$. Thus, these investigations suggest that endurance training may slow or even reverse the age-related changes in LV filling dynamics, thereby preserving filling patterns more similar to younger adults.

In agreement, there were no age-related differences in DFR at rest or at any of the matched HR during exercise (refer to Figure 15 and Table 2). However, this result was expected since there were no age-related differences in either SV or DT (which were used to calculate DFR). Additionally, although DFRmax was faster in group 20 compared to groups 50 and 60 , this was strictly due to the fact that group 20 was at a higher HRmax than groups 
50 and 60. Further, due to a limitation of the technique used to measure DFR in the present investigation, the separate phases which comprise DFR (isovolumic relaxation, early filling, diastasis and late atrial filling) as well as peak DFR could not be analyzed.

Similarly, there were no age-related differences in LVER at rest or at any of the matched HR during exercise (refer to Figure 16 and Table 2). However, this result was also expected since there were no age-related differences in either SV or LVET (which were used to calculate LVER). Additionally, although LVERmax was faster in group 20 compared to groups 50 and 60 , this was strictly due to the fact that group 20 was at a higher HRmax than groups 50 and 60.

Since no data to date are available regarding the relative contributions of DFR and LVER during incremental exercise to maximum as a function of age, direct comparisons could not be made to the literature. In the present investigation, when comparing the relative contributions of DFR and LVER at matched HR during exercise, DFR was faster than LVER in all four groups. For instance, at $150 \mathrm{bpm}$, in group 20; DFR was $40 \%$ faster than LVER, in group 40; DFR was 26\% faster than LVER, in group 50; DFR was $43 \%$ faster than LVER, and in group 60 DFR was $40 \%$ faster than LVER (refer to Figures 15, 16 and Table 2). Further, at maximum, in group 20; DFRmax was 57\% faster than LVERmax, in group 40; DFRmax was $49 \%$ faster than LVERmax, in group 50; DFRmax was $49 \%$ faster than LVERmax, and in group 60 DFRmax was $38 \%$ faster than LVERmax (Figures 15 and 16). This suggests that ET females rely to a greater extent on DFR than on LVER to augment their SV throught incremental exercise to maximum, regardless of age. Therefore, this 
provides further support for diastolic function, rather than systolic function as being the major determinant of an augmented cardiac function.

\section{Gender Differences in DFR and LVER}

Since limited data are available regarding the relative contributions of DFR and LVER during incremental exercise to maximum, comparisons of the findings in this investigation with published findings are limited. Fagard et al. (72) used imaging and Doppler ECG and observed that the early DFR (estimated from the velocity of LV relaxation and LV inflow pattern) in ET females was similar to that of age-matched control females. Similarly, when Spina et al. (256) examined sedentary males $(66 \pm 1 \mathrm{yr})$ and females $(64 \pm 1 \mathrm{yr})$ who underwent an endurance training program ( $3 \mathrm{mo}, 50 \mathrm{~min} \cdot \mathrm{d}^{-1}, 3-4 \mathrm{~d} \cdot \mathrm{wk}^{-1}$ at $80 \%$ of $\mathrm{HRmax}$ ), the older females did not display an increase in resting or in peak LV filling rates (counts $\cdot \mathrm{s}^{-1}$ ), or a decrease in TPFR, while the older males displayed these training-induced adaptations. These investigations suggest that females may not display the endurance training-induced augmentations in DFR which are observed in males.

The DFRmax of group 20 was $40 \%$ slower than the rate observed in a group of ET male cyclists (1132 vs $\left.1880 \mathrm{ml} \cdot \mathrm{sec}^{-1}\right)$, with no difference in maximal HR (190 bpm for males vs $192 \mathrm{bpm}$ for females) (105). The gender difference in DFRmax is explained by the larger SVmax (188 vs $125 \mathrm{ml}$ ) as well the slightly shorter DTmax (99 vs $111 \mathrm{msec}$ ) in the ET male cyclists compared to group 20. A larger SV would lead to a greater atrio-ventricular pressure gradient and thus, would help augment diastolic filling. Furthermore, while the DFRmax was $86 \%$ greater than the LVERmax in the ET male cyclists ( $\left.1880 \mathrm{vs} 1010 \mathrm{ml} \cdot \mathrm{sec}^{-1}\right)$, the DRFmax 
was only $57 \%$ greater than the LVERmax in the ET females $\left(1132\right.$ vs $\left.720 \mathrm{ml} \cdot \mathrm{sec}^{-1}\right)$. Thus, the greater SVmax as well as the progressive increase in SV up to maximum, would appear to be more reliant on the DFR which greatly exceeds the LVER in the ET males, with the ET females relying to a lesser extent on the DFR (105).

Warburton et al. (289), also examined the relative contributions of DFR and LVER during incremental exercise to maximum in younger ET males. Although the DFRmax of group 20 was $26 \%$ slower than the ET males from the Warburton et al. investigation (1132 \pm 40 vs $1532 \pm 36 \mathrm{ml} \cdot \mathrm{sec}^{-1}$ ), this gender difference was less than that observed in the Gledhill et al. (105) investigation, and when expressed relative to body mass, was only $14 \%$ slower in the ET females $\left(18.85 \pm 0.87\right.$ vs $\left.21.87 \pm 0.53 \mathrm{ml} \cdot \mathrm{kg}^{-1} \cdot \mathrm{sec}^{-1}\right)$. Further, the LVERmax of group 20 was $27 \%$ slower than the ET males from the Warburton et al. investigation ( $720 \pm$ 37 vs $\left.984 \pm 28 \mathrm{ml} \cdot \mathrm{sec}^{-1}\right)$, although when expressed relative to body mass was only $15 \%$ slower in the ET females $\left(11.97 \pm 0.60 \mathrm{vs} 14.05 \pm 0.48 \mathrm{ml} \cdot \mathrm{kg}^{-1} \cdot \mathrm{sec}^{-1}\right)$. The mechanisms responsible for the gender difference in DFRmax and LVERmax were the smaller SVmax observed in the ET females compared to the ET males (125.4 \pm 4.7 vs $159.6 \pm 4.0 \mathrm{ml}$ ), in addition to the ET females prolonged DTmax and LVETmax. The DFRmax was $56 \%$ greater than the LVERmax in the ET males ( $\left.1532 \pm 36 \mathrm{vs} 984 \pm 28 \mathrm{ml} \cdot \mathrm{sec}^{-1}\right)$, and $57 \%$ greater than the LVERmax in the ET females (1132 \pm 40 vs $\left.720 \pm 37 \mathrm{ml} \cdot \mathrm{sec}^{-1}\right)$. Thus, it appears that although ET males have a larger SVmax than ET females, which is due to the lower DFRmax and LVERmax in ET females, both males and females rely to a greater extent on diastolic function than on systolic function to augment their SVmax. Furthermore, it is possible that 
similar to previous observations of males (152), the mechanism responsible for the enhanced diastolic function in ET females is their elevated BV.

Blood Pressure, MAP and TPR in ET Individuals and the Relationship to

\section{Age and Gender}

Investigators have observed lower SBP and DBP at all levels of exercise in ET compared to NA males and females $(145,181,227,234)$. Further, a decreased TPR has been observed in ET males and females $(82,105,128,152,181,309)$, which results in a decreased afterload, and in turn, an enhanced blood flow and SV (11). For instance, Fleg et al. (82) found greater decreases in systemic vascular resistance from rest to peak exercise, as well as at peak exercise in older ET males compared to the age-matched NA males.

In the present investigation, SBP, and DBP were measured, while MAP (MAP = DBP $+1 / 3 P P)($ where $P P=S B P-D B P)$ and $T P R$ were calculated $(T P R=M A P / Q)$. These measures displayed the typical pattern observed during incremental exercise. The SBP and MAP increased linearly throughout incremental exercise as a result of an increasing $Q$ (refer to Figures $17,18,21$, and 22). The DBP decreased from rest to $110 \mathrm{bpm}$ and then plateaued (refer to Figures 19 and 20). The TPR decreased linearly as a result of vasodilation in the active skeletal muscles (refer to Figures 23 and 24).

\section{Age-Related Changes in BP, MAP and TPR}

A larger age-related increase in SBP has been observed in females, which is mediated by a larger age-related increase in systemic vascular resistance compared to males $(51,102$, $107,181,190,191)$. For instance, an investigation on peripheral vascular function in younger 
and older males and females, indicated a greater age-related reduction in maximal calf vasodilatory capacity, which was associated with a greater age-related increase in BP in both ET and NA females compared to similarly trained males, although not in those females treated with HRT (181).

No significant group differences were evident for SBPmax (refer to Figures 17, 18 and Table 2). The DBPmax was $24 \%$ higher in group 60 compared to group 20 (refer to Table 2). The higher DBPmax in group 60 was reflected in a 13\% higher MAPmax in this group compared to group 20. Further, MAPmax was $13 \%$ higher in group 60 compared to group 40 and 10\% higher in group 60 compared to group 40 (refer to Figure 21). One mechanism responsible for the higher MAPmax in these groups is the higher TPRmax. The TPRmax was $71 \%$ higher in group 60 compared to group $20,41 \%$ higher in group 50 compared to group 20 and 39\% higher in group 60 compared to group 40 (refer to Table 2). Thus, in agreement with the literature, it appears that ET females (not using HRT) display age-related increases in DBPmax, MAPmax and TPRmax.

\section{Gender Differences in BP, MAP and TPR}

Investigators have observed lower SBPmax in younger females compared to agematched males (130, 181). Similarly, group 20 had a lower SBPmax than the ET males from the Warburton et al. (289) investigation (182 \pm 5 vs $194 \pm 3 \mathrm{mmHg}$ ). Although there were no differences in DBPmax for the ET females and ET males ( $66 \pm 2$ vs $67 \pm 2 \mathrm{mmHg}$ ), the lower SBPmax observed in the ET females, resulted in a lower MAPmax, compared to the ET males ( $104 \pm 3$ vs $110 \pm 1 \mathrm{mmHg}$ ). Furthermore, the ET females had a higher TPRmax 


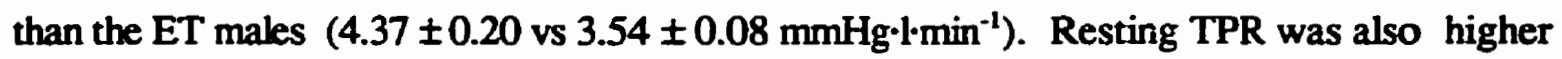

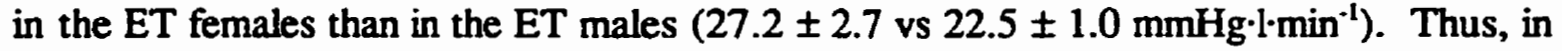
agreement with the literature, in comparison to ET males, ET females have a lower SBPmax and MAPmax, and a higher TPRmax. 


\section{CHAPTER VI}

\section{CONCLUSIONS, LIMUTATIONS AND RECOMMENDATIONS}

\section{Conclusions}

\section{Based on the findings from the present investigation, it may be concluded that:}

1. Cardiac function at rest and during submaximal exercise is maintained as a function of age in ET females.

2. $\mathrm{VO}_{2}$ max declines progressively at an approximate rate of $0.51 \mathrm{ml} \cdot \mathrm{kg}^{-1} \cdot \mathrm{min}^{-1} \cdot \mathrm{yr}^{-1}$ in $\mathrm{ET}$ females.

3. The decline in $\mathrm{VO}_{2} \max$ is due to a decrease in $\mathrm{Qmax}$, while $\mathrm{a}-\overline{\mathrm{v}} \mathrm{DO}_{2} \max$ is maintained as a function of age in ET females.

4. The decrease in $Q \max$ is due to a decline in HRmax, while SVmax is maintained as a function of age in ET females.

5. The maintenance of $\mathrm{SV} \max$ is due to a maintenance in $\mathrm{BV}$ as a function of age in ET females.

6. SV increases progressively throughout incremental work rates to maximum in ET females 20-29 yr, but reached a plateau at submaximal HR in ET females 40-63 yr.

7. At rest and during exercise at matched HR, LVET, PEP and DT are maintained with age in ET females.

8. At matched HR during exercise, the duration of LVET is longer than the duration of DT in ET females. 
9. At rest and during exercise at matched HR, DFR and LVER are maintained with age in ET fermales.

10. At matched HR during exercise, DFR is faster than LVER in ET females.

11. SBPmax is maintained as a function of age in ET females.

12. DBPmax increases progressively as a function of age in ET females.

13. The increase in DBPmax is reflected in a progressive increase in MAPmax as a function of age in ET females.

14. The increase in MAPmax is reflected in a progressive increase in TPRmax as a function of age in ET females.

Based on the findings from the present investigation of younger ET females compared to age-matched ET males from the investigation by Warburton et al. (289), it may also be concluded that:

1. ET males have a higher [Hb], oxygen carrying capacity and oxygen transport than ET females.

2. ET males have a greater $\mathrm{VO}_{2} \max$ than $\mathrm{ET}$ females.

3. The greater $\mathrm{VO}_{2} \max$ of ET males is due to their larger $\left(\max\right.$, while $\mathrm{a}-\mathrm{v} \mathrm{vO}_{2} \max$ is not gender related.

4. The larger Qmax of ET males is due to their elevated SVmax, while HRmax is not gender related.

5. The elevated SVmax of ET males is due to their larger BV.

6. At rest, ET males require more time for LVET, and less time for both DT and PEP 
than ET females.

7. The prolonged LVET and subsequent shortened DT at rest in ET males compared to ET females is due to the larger BV of ET males.

8. ET males have a faster DFRmax than ET females.

9. The faster DFRmax of ET males is due to their elevated BV through SVmax.

10. ET males have a faster LVERmax than ET females.

11. The faster LVERmax of ET males is due to their elevated BV through SVmax.

12. The DFRmax of ET males and females is greater than the LVERmax of ET males and females.

13. When expressed relative to body mass, although $\mathrm{VO}_{2} \max ,[\mathrm{Hb}], \mathrm{BV}, \mathrm{DFRmax}$ and LVERmax are still significantly greater in ET males, the gender differences in Qmax and SVmax are no longer significant, and ET females have a larger a-vDO $\mathrm{O}_{2} \max$ than ET males.

14. ET males have a higher SBPmax than ET females.

15. DBPmax is not gender related.

16. ET males have a higher MAPmax than ET females.

17. ET females have a higher TPRmax than ET males. 


\section{Limitations}

The conclusions were made with the following limitations in mind:

1. The participants in the present investigation were selected from a specific subgroup of the population. They represent endurance trained females ranging in age from $19-63 \mathrm{yr}$, with a high maximal oxygen uptake based on their age group. They had all trained vigorously for at least $3 \mathrm{yr}$ and had competed at a provincial, national or international level. Therefore, generalizations beyond this population may not be appropriate.

2. The small number of participants involved in this investigation may not allow for generalizations to be made to the ET population as a whole.

3. Cross-sectional investigations identify only potential age effects. For instance, as pointed out by Epstein (68a), the inherent error introduced into all cross-sectional aging studies is that of "selection mortality", such that, "Subject's over the age of 75 represent biologically superior survivors from a cohort that has experienced at least $75 \%$ mortality". Although participants represented in the present investigation were not greater than $63 \mathrm{yr}$, it is possible that some of the older individuals were genetically endowed with superior health or cardiac function which allowed them to compete successfully in endurance events, which would thus, tend to minimize any observed effects of aging. 


\section{Recommendations}

Future investigations in this area may consider the following:

1. Conduct a similar investigation with individuals $80 \mathrm{yr}$ and older of both genders, who are healthy and physically active.

2. Conduct a similar investigation with ET males to characterize the responses and agerelated differences to ET females.

3. Conduct a similar study with children and adolescents (pre- and post-pubertal) of both genders to characterize the responses and differences to older individuals.

4. Conduct a similar study with age-matched females and compare OCA users with nonusers.

5. Conduct a similar study with age-matched postmenoupausal females and compare those who are taking HRT with those who are taking only estrogen and those who are not taking any exogenous hormones.

6. Conduct a study to examine a- $\mathrm{VDO}_{2} \max$ in both $\mathrm{ET}$ and NA males and females.

7. Determine whether a BV gene exists. 


\section{REEERENCES}

1. Adams, K.F., L.M.Vincen, S.M. McAllister, H. El-Ashmawy, and D.S. Sheps. The influence of age and gender on left ventricular response to supine exercise in asymtomatic normal subjects. Am. Heart J. 113:732-742, 1987.

2. Akgun N., N. Tartaroglu, F. Durusoy and E. Kocaturk. The relationship between the cahnges in physical fitness and in total blood volume in subjects having regular and measured training. J. Sports Med. 14:73-77, 1974.

3. Anholm, J.D., C. Foster, J. Carpenter, M.L. Pollock, C. Hellman and D.H. Schmidt. Effect of habitual exercise on left ventricular response to exercise, J. Appl. Physiol. 52: $1648-1651,1982$.

4. Arora R.R., J. Machael, M.E. Goldman, R.N. Butler, R. Gorlin, and S.F. Horowitz. Atrial kinetics and left ventricular diastolic filling in healthy elderly. J. Am. Coll. Cardiol. 9(6) 1255-1260, 1987.

5. Astrand, P.O. and K. Rodahl. Textbook of Work Physiology, 3rd Ed. New York: McGraw-Hill, 1986, pp. 237-238.

6. Astrand, I., P.O. Astrand, I. Hallback and A. Kilbom. Reduction in maximum oxygen uptake wih age. J. Appl. Physiol. 35:649-654, 1973.

7. Astrand, P.O., T.E. Cuddy, B. Saltin, and J. Stenberg. Cardiac output during submaximal and maximal work. J. Appl. Physiol. 19:268-274, 1964.

8. Becklake, M.R., H. Frank, G.R. Dagenails, G.L. Ostiguy and C.A. Guzman. Influence of age and sex on exercise cardiac output. J Appl. Physiol. 20(5)938-947, 1965.

9. Bevegard, S., A. Holmgren, and B. Jonsson. Circulatory studies in well trained athletes at rest and during heavy exercise, with special reference to stroke volume and the influence of body position. Acta. Physiol. Scand. 57:26-50, 1963.

10. Bezucha, G.R., M.C. Lenser, P.G. Hanson and F.J. Nagle. Comparison of hemodynamic responses to static and dynamic exercise. J. Appl. Physiol. 53, 1589-1593, 1982.

11. Blomquist, CG. Cardiovascular adaptations to physical training. Annu. Rev. Physiol. 45:169-189, 1983. 
12. Bonow R.O., D.F. Vitale, S.L. Bacharasch, B.J. Maron, and M.U. Green. Effects of aging on asynchronous left ventricular regional function and global ventricular filling in normal human subjects. J. Am. Coll. Cardiol. 11(1):50-58, 1988.

13. Boudoulas, H. Systolic time intervals. Eur. Heart J. 11:93-104, 1990.

14. Brecher, G.A. and A.T. Kissen. Relation of negative ventricular pressure to intraventricular volume. Circ. Res. 5:157-162, 1957.

15. Brotherhood, J.B. Brozovic, and L.G.C. Pugh. Hematological status of middle- and long-distance runners. Clin. Sci. Mol. Med. 48:139-145, 1975.

16. Brozek, J., F. Grande, J.T. Anderson, and A. Keys. Densitometric analysis of body composition: revision of some quantitative assumptions. Ann. N.Y. Acad. Sci., 110:113$140,1963$.

17. Bryner, R.W., R.C. Toffle, I.H. Ullich, and R.A. Yeater. Effect of low dose oral contraceptive on exercise performance. Br.J. Sports Med. 30:36-40, 1996.

18. Bryg R.J., G.A. Williams, and A.J. Labowitz. Effect of aging on left ventricular diastolic filling in normal subjects. Am. J. Cardiol. 59:971-974, 1987.

19. Buick, F., N. Gledhill, A.B. Froese, L. Spriet and E.C. Meyers. Effect of induced erythrocythemia on aerobic work capacity. J. Appl. Physioi. 48:636-642, 1980.

20. Burk E.J. Physiological effects of similar training programs in males and females. Res. Quart. Exerc. Sport 48:510-517, 1977.

21. Bush, J.M., J.S. Green, and S.F. Crouse. Estrogen replacement does not influence exercise-induced changes in total blood volume in postmenopausal women. Med. Sci. Sports Exerc. 28(5):S174, 1996 (abstract).

22. Buskirk, E.R. and J.L. Hodgson. Age \& aerobic power: The rate of change in men and women. Fed. Proc. 46:1284-1289, 1987.

23. Carrol, J.F., V.A. Convertino, C.E. Wood, J.E. Graves, D.T. Lowenthal and M.L. Pollock. Effect of training on blood volume and plasma hormone concentration in the elderly. Med. Sci. Sports Exerc. 27(1):79-84, 1995.

24. Carrol, J.F., V.A. Convertino, C.E. Wood, J.E. Graves, and M.L. Pollock. The influence of six months of exercise training on resting plasma volume and hormone levels 
in older men and women. Med. Sci. Sports Exerc. 24:S158, 1992.

25. Cheng, C.P., Y. Igarashi, and W.C. Little. Mechanism of augmented rate of left ventricular filling during exercise. Circ. Res. 7:9-19, 1992.

26. Chien, S., U. Shunichi, R.L. Simmons, F.F. McAllister, and M.I. Gregersen. Blood volume and age: repeated measures on normal men after 17 years. J. Appl. Physiol. 21:583-588, 1966.

27. Chrifie, R and D.H. Spodick. Densiotography: a new method for evaluation of cardiac performance at rest and during exercise. Am. Heart J. 83(4): 493-503, 1972.

28. Christensen C.L. and R.O. Ruhling. Physical characteristics of novice and experienced women marathon runners. Br. J. of Sports Med. 17:166, 1983.

29. Choong, C.Y., H.C. Herrmann, A.E. Weyman, and M.A. Fifer. Preload dependence of Doppler- derived indexes of left ventricular diastolic function. J. Am. Coll. Cardiol. 10:800-808, 1987.

30. Coggan, A.R., J. Spina, D.S. King, M.A. Rogers, M. Brown and P.M. Nemeth. Skeletal muscle adaptations to endurance training in 60-to 70-year old men and women. J. Appl. Physiol. 72:1780-1786, 1992.

31. Coggan, A.R., R.J. Spina, D.S. King; M.A. Rogers, -M. Brown, P.M. Nemeth, and J.O. Holloszy. Histochemical and enzymatic characteristics of skeletal muscle in masters athletes. J. Appl. Physiol. 68:1896-1901, 1990.

32. Cohen J.L., P.K. Gupta, E. Lichstein, and K.D. Chadda. The heart of a dancer. Am. J. Cardiol. 45:959-965, 1980.

33. Colan S.D., S.P. Sanders, D. Macpherson, and K.M. Borrow. Left ventricular diastolic function in athletes with physiological cardiac hypertrophy. J. Am. Coll. Cardiol. 6:545-549, 1985.

34. Constantini N.W. Clinical consequences of athletic amenorrhoea. Sports Med. 17:213-223, 1994.

35. Convertino, V.A. Blood volume: Its adaptations to endurance training. Med. Sci. Sports Exerc. 23:1338-1348, 1991a. 
36. Convertino, V.A., G.W. Mack, and E.R. Nadel. Elevated central venous pressure: a consequence of exercise training induced hypervolemia? Am. J. Physiol. 29:R273-R277, $1991 b$.

37. Convertino, V.A. Heart rate and sweat rate responses associated with exercsiseinduced hypervolemia. Med. Sci. Sports Exerc. 15:77-82, 1983.

38. Convertino, V.A., J. Hung, D.J. Goldwater, and R.F. Debusk. Cardiovascular responses to exercise in middle-aged men following ten days of bed rest. Circul. 65:134$140,1982$.

39. Conway, J., R. Wheeler, and R. Sannerstedt. Sympathetic nervous system activity during exercise in relation to age. Cardiovasc. Res. 5:577-581, 1971.

40. Costill D.L., W.J. Fink, L.H. Getchell, J.L. Ivy and F.A. Witzmann. Lipid metabolism in skeletal muscle of endurance trained males and females. J. Appl. Physiol. 47:787-791, 1979.

41. Costill D.L., J. Daniels, W. Evans, W.J. Fink, C. Krahenbuhl and B. Saltin. Skeletal muscle enzymes and fibre composition in male and female track athletes. J. Appl. Physiol. 40:149-154, 1976.

42. Costill D.L., and E. Winrow. Maximal oxygen consumption among marathon runners. Arch. Phys. Med. 51:317-320, 1970.

43. Cowan, M.M. and L.W. Gregory. Responses of pre and post-menopausal femaies to aerobic conditioning. Med Sci. Sports Exerc. 17(1):138-143, 1985.

44. Cox, D. "Differences in cardiac time intervals and stroke volume between trainind and untrained subjects during moderate to maximal exercise: a heart rate controlled study." (Thesis) North York: Graduate Program in Exercise and Sports Science, Sept. 1991.

45. Coyle, E.F., M.K. Hemmert, and A. K. Coggan. Maximal oxygen uptake relative to plasma volume expansion. Int. J. Sports Med. 11(2):116-119, 1990.

46. Crawford, M.H., M.A. Petru, and C. Rabinowitz. Effect of isotonic exercise training on left ventricular volume during upright exercise. Circul. 72:1237-1242, 1985.

47. Cunningham D.A., and J.S. Hill. Effect of training on cardiovascular responses to exercise in women. J. Appl. Physiol. 39:891-895, 1975. 
48. Cureton K., P. Bishop, P. Hutchinson, H. Newland, S. Viskery, and L. Zwiren. Sex differences in maximal oxygen uptake. Effect of equating hemoglobin concentration. Eur. J. Appl. Physiol. 54:656-660, 1986.

49. Cureton K.J. and P.B. Sparling. Distance running performance and metabolic responses to running in men and women with excess weight experimentally equated. Med Sci. Sports Exerc. 12(4):288-294, 1980.

50. Daggett, A., B. Davies, and L. Boobis. Physiological and biochemical responses to exercise following oral contraceptive use. Med. Sci. Sports Exerc. 15:174, 1983.

51. Daida H., T.G. Allison, R.W. Squires, T.D. Miller and G.T. Gau. Peak exercise blood pressure statified by age and gender in apparently healthy subjects. Mayo Clin. Proc. 71:445-452, 1996.

52. Daniels, J., G. Krahenbuhl, C. Foster, J. Gilbert, and S. Danials. Aerobic responses of female distance runners to submaximal and maximal exercise. Ann. NY Acad. Sci. (301): 726-733, 1977.

53. Davy, K.P. and D.R. Seals. Total blood volume in healthy young and older men. $J$. Appl. Physiol. 76(5):2059-2062, 1994.

54. Dehn, M.M. and R.A. Bruce. Longitudinal variation in maximal oxygen intake with age and activity. J. Appl. Fhysiol. 33(6):805-807, 1972.

55. DeSouza, MJ., M.S. Maguire, K.S. Rubin, and C.M. Maresh. Effects of menstrual phase and amenorrhea on exercise responses in runners. Med. Sci. Sports Exerc. 22, 575$580,1990$.

56. DeSouza, M.J., C.M. Maresh, M.S. Maguire, W.J. Kraemer, G. Flora-Ginter, and K.L. Goetz. Menstrual status and plasma vasopresin, renin activity and aldosterone exercise responses. J. Appl. Physiol. 67(2):736-743, 1989

57. Devereux, R.B., E.M. Lutas, P.N. Casale, P. Klingtield, R.R. Eisenberg, I.W. Hammond, D.H. Miller, G. Reis, M.H. Alderman and J.H. Laragh. Standaridization of Mmode echocardiography in left ventricular anatomic measurement. J. Am. Coll. Cardiol. 4:1222-1230, 1984.

58. Dill, D.B. and D.B. Costill. Calculation of percentage changes in the volumes of blood, plasma and red cells in dehydration. J. Appl. Physiol. 37(2):247-248, 1974a. 
59. Dill, D.B., K. Braithwaite, W.C. Adams, and E.M. Bernauer. Blood volume of middle-distance runners: effect of 2,300-m altitude and comparison with non- athletes. Med. Sci. Sports Exerc. 6:1-7, 1974b.

60. Dill, D.B., S. Robinson and J.C. Ross. A longitudinal study of 16 champion runners. J. Sports Med. Phys. Fit. 7:4-27, 1967.

61. Dombovy, M.L., H.W. Bonekat, T.J. Williams and B.A. Staats. Exercise performance and ventilatory response in the menstrual cycle. Med. Sci. Sports Exerc. 19:111-117, 1987.

62. Douglas, P.S., and M. O'Toole. Aging and physical activity determine cardiac structure and function in the older athlete. J. Appl. Physiol. 72(5):1969-1973, 1992.

63. Douglas, P.S., M.L. O'Toole, W.D.B. Hiller and N. Reichek. Left ventricular structure and function by echocardiography in ultra endurance athletes. Am. J. Cardiol. (58): 805-809, 1986.

64. Drinkwater, B.L. Athletic amenorrhea: a review. In: Exercise and Health, Human Kinetics Pub.: Champaign, ILL, 1984, pp. 120-131.

65. Drinkwater, B.L., S.M. Horvath and C.L. Wells. Aerobic power of women age 1068. J. Gerontol. 30:385-394, 1975.

66. Ehsani, A.A., T. Ogawa, T.R. Miller, R.J. Spina, and S.M. Jilka. Exercise training improves left ventricular systolic function in older men. Circul. 83:96-103, 1991.

67. Ekblom, B., and L. Hermansen. Cardiac output in athletes. J. Appl. Physiol. 25(5):619-625, 1968a.

68. Ekblom, B., P.O. Astrand, B. Saltin, J. Stenberg, B. Wallstrom. Effect of training on circulatory response to exercise. J. Appl. Physiol. 24(4):518-528, $1968 \mathrm{~b}$.

68a. Epstein, M. Effects of aging on the kidney. Fed. Proc. 38:168-172, 1979.

69. Evans, S.L., K.P. Davy, E.T. Stevenson, and D.R. Seals. Physiological determinants of a 10-km performance in highly trained female runners of different ages. J. Appl. Physiol. 78(5):1931-1941, 1995.

70. Fagard, R.H. Impact of different sports and training on cardiac structure and function. Cardiology Clinics. 10(2) :241-256, 1992. 
71. Fagard, R., C. Van Den Broeke, and A. Amery. Left ventricular dynamics during exercise in elite marathon runners. J. Am. Coll. Cardiol. 14:112, 1989.

72. Fagard, R.H., C. Van Den Broeke, L. Vanhees, J. Straessen and A. Amery. Noninvasive assessment of systolic and diastolic left ventricular function in female runners. Eur. Heart J. 8(12) :1305-1311, 1987.

73. Fagard, R., A Aubert, J. Staessen, E.V. Eynde, L. Vanhees and A. Amery. Cardiac structure and function in cyclists and runners: Comparitive echocardiographic study. $\mathrm{Br}$. Heart J. 52:124, 1984.

74. Falsetti H., C. Gisolfi, D. Lemon, J. Cohen, and B. Claxton. Non-invasive evaluation of left ventricular function in trained bicyclists. J. Sports Med. 22:199-206, 1982.

75. Fardy, P.S., C.M. Maresh, R. Abbott and T. Kristiansen. A comparison of habitual lifestyle, aerobic power and systolic time intervals in former and non-athletes. J. Sports Med. 18:287-299, 1978.

76. Fardy, P.S. The influence of physical activity on selected cardiac time components. J. Sports Med. Phys. Fitness 11:227-233, 1971.

77. Faulkner, J.A., G.F. Heigenhauser and M.A. Schork. The cardiac output - oxygen uptake relationship of men during graded bicycle exercise. Med. Sci. Sports Exerc. 9(3):148-154, 1977.

78. Fay L., Londeree B.R., Lafontaine T.P., and M.R. Volek. Physiological parameters related to distance running performance in female athletes. Med. Sci. Sports Exerc. $21: 319,1989$.

79. Finkelhor, R.S., L.J. Hanak, and R.C. Bahler. Left ventricular filling in endurance trained subjects. J. Am. Coll. Cardiol. 8:289-293, 1986.

80. Fleck S.J. Body composition of elite american athletes. Am. J. Sports Med. 11: 398403, 1983.

81. Fleg, J.L., F. O'Connor, G. Gerstenblith, L.C. Becker, J. Clulow, S.P. Schulman, E.G. Lakatta. Impact of age on the cardiovascular response to dynamic upright exercise in healthy men and women. J. Appl. Physiol. 78:3890-3900, 1995.

82. Fleg, J.L., S.P. Schulman, F.C. O'Connor, G. Gerstenblith, L.C. Becker, S. Fortney, A.P. Goldberg and E.G. Lakatta. Cardiovascular responses to exhaustive upright cycle 
exercise in highly trained older men. J. Appl. Physiol. 77(3): 1500-1506, 1994.

83. Fleg, J.L. and E.G. Lakatta. Role of muscle loss in the age-associated reduction in $\mathrm{VO}_{2}$ max. J. Appl. Physiol. 65:1147-1151, 1988.

84. Fleg, J. Alterations in cardiovascular structure and function with advancing age. Am. J. Cardiol. 57:33C-44C, 1986.

85. Forman, D.E., W.J. Manning, R. Hauser, E.V. Gervino, W.J. Evans, J.Y. Wei Enhanced left ventricular diastolic function associated with long-term endurance training. J. Gerontol. 47:M56-58, 1992.

86. Fortney, S.M., C. Turner, L. Steinman, T. Driscoll and C. Alfrey. Blood volume responses of men and women to bed rest. J. Clin. Pharmacol. 34:434-439, 1994.

87. Fortney, S.M., W.S. Beckett, A.J. Carpenter, J. Davis, H. Drew, N.D. LaFrance, J.A. Rock, C.G. Tankersley and N.B. Vroman. Changes in plasma volume during bed rest: effects of the menstrual cycle and estrogen administration. J. Appl. Physiol. 65(2):525$533,1988$.

88. Fortney, S.M., E.R. Nadel, C.B. Wenger and J.R. Bove. Effect of acute alterations of blood volume on circulatory performance in humans. J. Appl. Physiol. 50:292-298, 1981.

89. Fortney, S.M. and L.C. Senay. Ex̂ect of training and heat acclimatation on exercise responses of sedentary females. J. Appl. Physiol. 46(5):978-984, 1979.

90. Frantz, A.G. and M.T. Rabkin. Effects of estrogen and sex differences on secretion of human growth hormone. J. Clin. Endocr. 25: 1470-1480, 1965.

91. Freedson, P.S. The influence of hemoglobin on exercise cardiac output. Int. J. Sports Med. 2:81-86, 1981.

92. Freedson, P.S., V.L. Katch, S. Sady, and A. Weltman. Cardiac output differences in males and females during mild cycle ergometer exercise. Med. Sci. Sports Exerc. 11(1):16-19, 1979.

93. Freund, B.J., J.R. Claybaugh, M.S. Dice, and G.M. Hashiro. Hormonal and vascular fluid responses to maximal exercise in trained and untrained males. J. Appl. Physiol. 63(2):669-675, 1987. 
94. Frick, M.H., R.O. Elovainio, and T. Somer. The mechanism of bradycardia evoked by physical training. Cardiologica 51: 46-54, 1967.

95. Fuchi, T., K. Iwaoka, M. Higuchi, and S. Kobayashi. Cardiovascular changes associated with decreased aerobic capacity and aging in long-distance runners. Eur. $J$. Appl. Physiol. 58:884-889, 1989.

96. Gaebelein, C.J. and L.C. Senay, Jr. Vascular volume dynamics during ergometry exercise at different menstrual phases. Eur. J. Appl. Physiol. 50:1-11, 1982.

97. Gardin, J.M., W.L. Henry, D.D. Savage, J.H. Ware, C. Burn, J.S. Borer. Echocardiographic measurement in normal subjects: evaluation of an adult population without clinically apparent heart disease. J. Clin. Ultrasound 7:439-447, 1979.

98. George, K.P., Wolfe, L.A., Burggraf, G.W., and R. Norman. Electrocardiographic and echocardio-graphic characteristics of female athletes. Med. Sci. Sports Exerc. 27(10):1362-1370, 1995.

99. George K.P., L.A. Wolfe, and G.W. Burggraf. The athletic heart syndrome: A critical review. Sports Med. 11:330-331, 1991.

100. Gerstenblith, G., D.G. Renlund, and E.G. Lakatta. The cardiovascular response to exercise in younger and older men. Fed. Proc. 46:1834-1839, 1987.

101. Gerstenblith, G., J. Frederiksen, F.C.P. Yin, N.J. Fortuin, E.G. Lakatta, and M.D. Weisfeldt. Echocardiographic assessment of a normal adult aging population. Circul. 56(2):273-277, 1977.

102. Gerstenblith, G., E.G. Lakatta, and M.L. Weisfeldt. Age changes in myocardial function and exercise response. Prog. Cardiovasc. Dis. 19:1-21, 1976.

103. Gillen, C.M., R. Lee, G.W. Mack, C.M. Tomarelli, T. Nishiyasu and E.R. Nadel. Plasma volume expansion in humans after a single intense exercise protocal. $J$. Appl. Physiol. 71:1914-1920, 1991.

104. Ginzton, L.E., R. Conant, M. Brizendine, and M.M. Laks. Effects of long-term high intensity aerobic training on left ventricular volume during maximal upright exercise. $J$. Am. Coll. Cardiol. 14:364-361, 1989.

105. Gledhill, N., D. Cox, and V. Jamnik. Endurance athletes' stroke volume does not plateau: major advantage is diastolic function. Med. Sci. Sports Exerc. 26(9):1116-1121, 
1994.

106. Gledhill, $\mathbf{N}$. The influence of altered blood volume and oxygen transport capacity on aerobic performance. In: Exercise and Sports Sciences Review, R. Terjung and P. Gollinick (eds.), vol. 13. Macmillan, New York, pp 75-94, 1985.

107. Gleim, G.W., N.S. Stachenfeld, N.L. Coplan, and J.A. Nichols. Gender differences in the systolic blood presure response to exercise. Am. Heart J. 121:524-530, 1991.

108. Goodman, J.M., M.J. Plyley, C.A. Lefkowitz, P.P. Liu, and R.R. McLaughlin. Left ventricular functional response to moderate and intense exercise. Can J. Appl. Sport Sci. 16(3):204-209, 1991.

109. Graettinger, W.F. The cardiovascular response to chronic physical exertion and exercise training: an echocardiographic review. Am. Heart J.108:1014-1018, 1985.

110. Granath, A., and T. Strandell. Relationships between cardiac output, stroke volume and intracardiac pressures at rest and during exercise in supine position and some anthropometric data in healthy old men. Acta. Med. Scand. 176:447-466, 1964.

111. Granger, C.B., M.K. Karimeddini, V.E. Smith, H.R. Shapiro, A.M. Katz, and A.L. Riba. Rapid ventricullar filling in left ventricular hypertrophy: I. Physiologic hypertrophy. J. Am. Coll. Cardiol. 5:862-868, 1985.

112. Gray, M.J., K.S. Strausfeldt, M. Watanbe, E.A.H. Simms and S. Solomon. Aldosterone secretory rates in the normal menstrual cycle. J. Clin. Endocrinol. Metab. 28:1269-1275, 1968.

113. Green, H.J., J.A. Thomson, M.E. Ball, R.L. Hughson, M.E. Houston and M.T. Sharratt. Alterations in blood volume following short-term supramaximal exercise. $J$. Appl. Physiol. 56:145-149, 1984.

114. Green, J.S., F.S. Crouse, and M. Matte. Estrogen replacement influences peak cardiac output in exercise trainined postmenopausal women. Med. Sci. Sports Exerc. 28(5):S169, 1996 (abstract).

115. Grimby, G. and B. Saltin. Cardiac output during submaximal and maximal exercise in active middle-aged athletes. J. Appl. Physiol. 21(4):1150-1156, 1966.

116. Grollman, A. The determination of the cardiac output of man by the use of acetylene. Am. J. Physiol. 88:432-445, 1929. 
117. Hagberg, J.M., W.K. Allen, D.R. Seals, B.F. Hurley, A.A. Ehsani, and J.O. Holloszy. A hemo-dynamic comparisons of young and older endurance athletes during exercise. J. Appl. Physiol. 58(6): 2041-2046, 1985.

118. Hanley, P.C., A.R. Zinsmeister, I.P. Clements, A.A. Bove, M.L. Brown, and R.J. Gibbons. Gender related differences in cardiac responses to supine exercise assessed by radionucleotide angiography. J. Am. Coll. Cardiol. 13:624-629, 1989.

119. Harrison, M.H. Effects of thermal stress and exercise on blood volume in humans. Physiol. Rev. 65:149-209, 1985.

120. Harrison T.R., K. Dixon, P.O. Russell, Jr., P.S. Bidwai and H.N. Coleman. Relation of age to the duration of contraction, ejection, relative of normal human heart. Am. Heart $J .67(2): 189-199,1964$.

121. Hartley, L., G. Grimby, A. Kilbom, N. Nilson, I. Astrand, B. Ekblom, and B. Saltin. Physical training in sedentary middle-aged and older men III: Cardiac output and gas exchange at submaximal and maximal exercise. Scand. J. Clin. Lab. Investig. 24:335$344,1969$.

122. Heath, G.W., J.M. Hagberg, A.A. Ehsani, and J.O. Holloszy. A physiological comparison of young and older endurance athletes. J. Appl. Physiol. 51(3):634-640, 1981.

123. Higginbothman, M.B., K.G. Morris, R.S. Williams, P.A. McHale, R.E. Coleman, F.R. Cobb. Regulation of stroke volume during submaximal and maximal upright exercise in normal man. Circ. Res. 58:281-291, 1986a.

124. Higginbothman, M.B., K.G. Morris, R.S. Williams, and F.R. Cobb. Physiological basis for the age-related decline in aerobic work capacity. Am. J. Cardiol. 51:1374-1379, 1986b.

125. Higginbothman, M.B., K.G. Morris. R.E. Coleman and F.R. Cobb. . Sex-related differences in the normal cardiac response to upright exercise. Circul. 70:357-366, 1984.

126. Hirota, Y. A clinical study of left ventricular relaxation. Circul. 62:756-763, 1980.

127. Holmgren, A.F., Mossfeldt, T. Sjostrand, and G. Strom. Effect of training on work capacity, total hemoglobin, blood volume, heart volume and pulse rate in recumbent and upright position. Acta. Physiol. Scand. $50: 72-83,1960$. 
128. Hopper, M.K., A.R. Coggan, and E.F. Coyle. Exercise stroke volume relative to plasma-volume expansion. J. Appl. Physiol. 64:404-408, 1988.

129. Hopkins, M.G., R.J. Spina and A.A. Ehsani. Enhanced $\beta$-adrenergic mediated cardiovascular response in endurnace trained athletes. J. Appl. Physiol. 80(2):516-521, 1996.

130. Hossack, K.F. and R.A. Bruce. Maximal cardiac function in sedentary normal men and women: comparison of age related changes. J. Appl. Physiol. 53:799-804, 1982.

131. Ikaheimo, M.J., I.J. Palatsi, and J.T. Takkunen. Noninvasive evaluation of the athletic heart: Sprinters vs. endurance runners. Am. J. Cardiol. 44:24-30, 1979.

132. Jackson, A.S, L.T. Wier, G.W. Ayers, E.F. Beard, J.E. Stuteville, and S.N. Blair. Changes in aerobic power of women, ages 20-64 yr. Med. Sci. Sports Exerc. 28(7):884$891,1996$.

133. Jackson, N.L., J.S. Green, and S.F. Crouse. Left ventricular structural adaptations to endurance exercise in postmenopausal women are not influenced by estrogen replacement. Med. Sci. Sports Exerc. 28(5):S169, 1996 (abstract).

134. Jackson, A.M., M.L. Pollock, and A. Ward. Generalized eqautions for predicting body density of women. Med. Sci. Sports Exerc. 12:175-182, 1980.

135. Jiang, C., P.M. Sarrel, D.C. Lindsay, P.A. Poole-Wilson and P. Collins. 17- $\beta$ Estradiol inhibits contraction in isolated rat coronary arteries by an EDRF-independent mechanism. Circul. 82 (Suppl):III-489, 1990 (abstract).

136. Jones P.P. , K.P. Davy, M.J. Reiling and D.R. Seals. Maintenance of total blood volume with age in female endurance athletes. Med. Sci. Sports Exerc. 28(5):S34, 1996 (abstract).

137. Jones, W.B. and G.L. Foster. Determination of duration of left ventricular ejection in normal young men. J. Appl. Physiol. 19(2):279-283, 1964.

138. Joyner, M.J. Physiological limiting factors and distance running: influence of gender and age on record performances. In: J. Holloszy (Ed.) Exercise and Sport Sciences Reviews, Baltimore, MD.: Williams and Wilkins, vol. 21, 1993, pp. 103-135.

139. Julius, S., A. Amery, L.S. Whitlock and J. Conway. Influence of age on the hemodynamic response to exercise. Circul. 36:222-230, 1967. 
140. Kanstrup I.L., B. Ekblom, J. Marving, and P.F. Hoilund-Carlson. Acute plasma volume expansion: Left ventricular hemodynamics and endocrine function during exercise. J. Appl. Physiol. 73:1791-1796, 1992.

141. Kanstrup, I.L., and B. Ekblom. Blood volume and hemoglobin concentration as determinants of maximal aerobic power. Med. Sci. Sports Exerc. 16(3):256-262, 1984.

142. Kanstrup, I.L and B. Ekblom. Acute hypervolemia, cardiac performance and aerobic power during exercise. J. Appl. Physiol. 52(5) 1186-1191, 1982.

143. Kasch, F.W., J.L. Boyer, S.V. Camp, F. Netth, L.S. Verity, and J.P. Wallace. Cardiovascular changes with age and exercise. A 28-year longitudinal study. Scand. J. Med. Sci. in Sport 5(3):147-51, 1995.

144. Katch, F. and W.D. McArdle. Nutrition, weight Control and Exercise, 2nd Ed. Philadelphia: Lea and Febinger, 1983.

145. Keul J., H.H. Dickhuth, M. Lehman and J. Staiger. The athlete's heart: Hemodynamica and structure. Int. J. Sports Med. 3(suppl 1):33-43, 1982.

146. Kiezer, H.A. and A.D. Rogol. Physical exercise and menstrual cycle alterations: What are the mechanisms? Sports Med. 10: 218-235, 1990.

147. Kilblom, A. and I. Astrand. Physical training with submaximal intensities in women. II. Effect on cardiac output. Scand. J. Clin. Lab. Invest. 28:163-175, 1971.

148. Kitzman D.W., K.H. Sheikh, P.A. Beere, J.L. Philips, and M.B. Higginbotham. Age-related alterations in Doppler left ventricular filling indexes in normal subjects are independent of left ventricular mass, heart rate, contractility and loading conditions. $J$. Am. Coll. Cardiol. 18(5):1243-1250, 1991.

149. Kjellberg, S.R., U. Rudhe, and T. Sjostrand. Increase of the amount of hemoglobin and blood volume in connection with physical training. Acta. Physiol. Scand. 19:146-151, 1949.

150. Kollias, J., H.L. Barlett, J. Mendez, B. Franklin. Hemodynamic responses of well trained women athletes to graded treadmill exercise. J. Sports Med. 18(4): 365-372, 1978.

151. Komi P.V. and J. Karlsson. Skeletal muscle fibre types, enzyme activities and physical performance in young males and females. Acta. Physiol. Scand. 103:210-218, 
1978.

152. Krip, B., N. Gledhill, V. Jamnik, N. Card, and D. Warburton. Enhanced diastolic filling of endurance trained athletes is due primarily to their higher blood volume. Med. Sci. Sports Exerc. 29(11):1469-1476, 1997.

153. Krzeminski, K, W. Niewiadomski, K. and Nazar. Dynamics of changes in the cardiovascular response to submaximal exercise during low intensity endurance training with particular reference to systolic time intervals. Eur. J. Appl. Physiol. 377-384. 1989.

154. Lamont, L. Effects of training on echocardiographic dimensions and systolic time intervals in women swimmers. J. Sports Med. 20:397-404, 1980.

155. Lakatta, E.G., F. O'Connor, S. Sculman, G. Gerstenblith, L. Becker, and J.L. Fleg. Cardiac volumes at rest and during cycle exercise in healthy men of a broad age range: effect of fitness matching. Fed. Proc. 5:A766, 1991 (abstract).

156. Lakatta, E.G., J.H. Mitchell, A. Pomerance, and G.G. Rowe. Human aging: changes in structure and function. J. Am. Coll. Cardiol. 10(2):42A-47A, 1987.

157. Lakatta, E.G. Age-related alterations in the cardiovascular response to adrenergic mediated stress. Fed. Proc. 39: 3173-3177, 1980.

158. Lakatta, E.G. Alterations in the cardiovascular system that occur in advanced age. Fed. Proc. 38:163-167, 1979.

159. Lakatta, E.G., G. Gerstenblith, C.S. Angell, N.W. Shock, and M.L. Weisfeldt. Prolonged contraction duration in aged myocardium. J. Clin. Investig. 55:61-68, 1975a.

160. Lakatta, E.G., G. Gerstenblith, C.S. Angell, N.W. Shock and M.L. Weisfeldt. Diminished inotropic response of aged myocardium to catecolamines. Circ. Res. 36:262$269,1975 b$.

161. Lance, V.Q. and D.H. Spodick. Systolic time intervals utilizing ear densitography: advance and reliability for stress testing. Am. Heart J. 94(1):62-66, 1977.

162. Lebrun, CM. Effects of the menstrual cycle phase on athletic performance. Med. Sci. Sports Exerc. 27(3): 437-444, 1995.

163. Lebrun, C.M. The effect of the phase of the menstrual cycle and the birth control pill on athletic performance. Clin. In Sports Med. 13(2):419-441, 1994. 
164. Lehtovirta, P., J. Kuikka, and T. Pyorala. Hemodynamic effects of oral contraceptives during exercise. Int. J. Gynaecol. Obstet. 15:35-37, 1977.

165. Levine, B.D. Regulation of central blood volume and cardiac filling in endurance athletes: the Frank-Starling mechanism as a determinanat of orthostatic intolerance. Med. Sci. Sports Exerc. 25(6):727-732, 1993.

166. Levine, B.D., L.D. Lane, J.C. Buckey, D.B. Friedman and C.G. Blomquist. Left ventricular pressure-volume and Frank-Starling relations in endurance athletes: implications of orthostatic tolerance and exercise performance. Circul. 84:1016-1023, 1991.

167. Levy, W.C., M.D. Cerqueira, I.B. Abrass, R.S. Schwartz, and J.R. Stratton. Endurance exercise training augments diastolic filling at rest and during exercise in healthy young and older men. Circul. 88:116-126, 1993.

168. Lewis R.P. Systolic time intervals and other graphic methods. In: Diagnostic Procedures in Cardiology: A Clinician's Guide. Chicago, 1985, pp. 91-103.

169. Lewis, R.P., S.E. Rittgers, W.F. Forester, and H. Boudoulas. A critical review of systolic time intervals. Circul. 56(2):146-157, 1977.

170. Longhurst J.C, A.R. Kelly, W.J. Gonyea, and J.H. Mitchell. Echocardiographic left ventricular-masses in distance runners and weight lifters. J. Appl. Physiol. 48:154-162, 1980.

171. Lombard W.P., and O.M. Cope. Sex-differences in heart action: I. Duration of systole. Am. J. Physiol. 37-41, 1927a.

172. Lombard W.P., and O.M. Cope. Sex differences in heart action: II. Heart rate. Am. J. Physiol. 42-46, $1927 \mathrm{~b}$.

173. Lombard W.P., and O.M. Cope. The duration of systole of the left ventricle of man. Am. J. Physiol. 263-268, 1926.

174. Luetkemeier, M.J., K.M. Flowers, D.R. Lamb. Spirnolactone administration and training induced hypervolemia. Int. J. Sports Med. 295-300, 1994.

175. Luotola $\mathrm{H}$. Blood pressure and hemodynamics in post-menopausal women during estradiol-17ß substitution. Ann. Clin. Res. 15(Suppl38)1-121, 1983. 
176. Magorien, D.J., P. Shaffer, C. Bush, R.D. Magorien, A.J. Kolibash, D.V. Underferth, T.M. Bashore. Hemodynamic correlation for timing intervals, ejection rate and filling rate derived from radionucleotide angiographic volume curve. Am. J. Cardiol. 53:567-571, 1984.

177. Maher, J.T., G.A. Beller, B.J. Ransil, and L.H. Hartley. Systolic time intervals during submaximal and maximal exercise in man. Am. Heart J. 87(3):334-342, 1974.

178. Makarides, L., G.L. Hiegenhausser, and N.L. Jones. High intesity endurance training in 20- to 30- and 60- to 70-yr old healthy men. J. Appl. Physiol. 69(6):1792$1798,1990$.

179. Mann, D.L., B.S. Denenberg, A.K. Gash, P.T. Makler and A.A. Bove. Effects of age on ventricular performance during graded supine exercise. Am. Heart J. 111:108-115, 1986.

180. Marti, B., and H. Howald. Long-term effects of physical training on aerobic capacity: controlled study of former elite athlete. J. Appl. Physiol. 69:1451-1459, 1990.

181. Martin III, W.H., T. Ogawa, W.M. Kohrt, M.T. Malley, E. Korte, P.S. Keiffer, and K.B. Schechtman. Effects of aging, gender, and physical training on peripheral vascular function. Circul. 84:654-664, 1991.

182. Martin D.E. and D.F. May. Pulmonary function characteristics in elite women distance runners. Int. J. Sports Med. 8(Suppl):84-90, 1987

183. Martin D.E., D.F. May and S.P. Pilbeam. Ventilation limitations to performance among elite male distance runners. In: D.M. Landers (Ed.) Sport and Elite Performers, Champaign, IL: Human Kinetics, 1984, pp. 121-131.

184. Martin B.J., K.E. Sparks, C.W. Zwillich, and J.V. Weil. Low exercise ventilation in endurance athletes. Med. Sci. Sports Exerc. 11:181-185, 1979.

185. Martin, C.E., M.D. James, J.A. Shaver, M.E. Thompson, P.S. Reddy, and J.J. Leonard. Direct correlation of external systolic time intervals with internal indicies of left ventricular function in man. Circul. 43:419-431, 1971.

186. Matsuda, M., Y. Sugishita, S. Koeseki, I. Ito, T. Akastsuka, and K. Takamatsu. Effects of exercise on left ventricular diastolic filling in athletes and non-athletes. J. Appl. Physiol. 55:323-328, 1983. 
187. McCole, S.D., M.D. Brown, G.E. Moore, J.M. Zmuda, J. Cwynar, and J.M. Hagberg. Maximal exercise cardiovascular hemodynamics in postmenopausal women are independent of hormone replacement therapy. Med. Sci. Sports Exerc. 29(5): S11, 1997 (abstract).

188. McElvaney, G.N., S.P. Blackie, N.J. Morrison, M.S. Fairban, P.G. Wilcox, and R.L. Pardy. Cardiac output at rest and during exercise in elderly subjects. Med. Sci. Sports Exerc. 21(3):293-298, 1989.

189. McGrath M.W. and M.L. Thompson. The effects of age, body size and lung volume changes on alveolar-capillary permeability and diffusing capacity in man. J. Physiol. 16:572-582, 1959.

190. Michelsen S. and J.E. Otterstad. Blood pressure responses during maximal exercise in apparently healthy men and women. J. Internal Med. 227:157-163, 1990.

191. Michelsen S., M. Hurlen, M. Stugaard and J.E. Otterstad. Influence of age on physical performance, heart rate and systolic blood pressure during exercise in apparently healthy women. Scand. J. Clin. Lab. Invest. 49:97-102, 1989.

192. Mier C.M., M.A. Domenick, N.S. Turner and J.H. Wilmore. Changes in stroke volume and maximal aerobic capacity with increased blood volume in males and females. J. Appl. Physiol. 80(4):1180-1186, 1996.

193. Miller, T.R., C.J. Stray-Gundersen, R.M. Peshock, J. Katz, and J.H. Mitchell. Left ventricular diastolic filling and its association with age. Am. J. Cardiol. 58:531-535, 1986.

194. Mitchell, J.H., C. Tate, P. Raven, F.Cobb, W. Kraus, R. Moreadith, M. O'Toole, B. Saltin, and N. Wenger. Acute response and chronic adaptation to exercise in women. Med. Sci. Sports Exerc. 24(suppl):S258-S265, 1992.

195. Miyamoto, Y., J. Higuchi, Y. Abe, Y. Huira, Y. Nakazono, and T. Mikami. Dynamics of cardiac output and systolic time intervals in supine and upright exercise. $J$. Appl. Physiol. 55(6)1674-1681, 1983.

196. Miyatake, K., M. Okamoto, N. Kintosh, M. Owa, I. Nakasone, H. Sakakibara, and Y. Nimura. Augmentation of atrial contribution to left ventricular inflow with aging as assesd by intracardiac Doppler flowmetry. Am. J. Cardiol. 53:586-589, 1984. 
197. Montoye, H.J., P.W. Willis, G.E. Howard, J.B. Keller. Cardiac pre-ejection period: Age and sex comparisons. J. Gerontol. 26: 208-216, 1971.

198. Morganroth J., B.J. Maron, W.L. Henry, and S.E. Epstein. Comparitive left ventricular dimensions in trained athletes. Ann. Internal Med. 82:521, 1975.

199. Morrison D.A., T.W. Boydon, R.W. Pamenter, B.J. Freund, W.A. Stini, R. Harrington, and J.H. Wilmore. Effects of aerobic training on exercise tolerance and echocardiographic dimensions in untrained post-menopausal women. Am. Heart J. 112:561, 1986.

200. Mumford, M. and R. Prakash. Electrocardiographic and echocardiographic characteristics of long-distance runners. Comparison of left ventricular function with age and sex matched controls. Am. J. Sports Med. 9:23-28, 1981.

201. Nixon, J.V., H. Hallmark, K. Page, P.R. Raven, and J.H. Mitchell. Ventricular performance in human hearts aged 61-73 years. Am. J. Cardiol. 56:932-937, 1985.

202. Notolovitz, M., C. Zauner, L. Mckenzie, Y. Suggs, C. Fields and C. Kitchens. The effect of low dose contraceptives on cardiorespiratory function, coagulation, and lipids in exercising young women: A preliminary report. Am. J. Obstet. Gynecol. 156:591-598, 1987.

203. Notolovitz, M., C. Fields, K. Caramelli, M. Dougherty, and A.L. Schwartz. Cardiorespiratory fitness evaluation in climacteric women: Comparison of two methods. Am. J. Obstet. Gynecol. 154:1009-1013, 1986.

204. Nygaard E. Skeletal muscle fibre characteristics in young women. Acta. Physiol. Scand. 112:299-304, 1981.

205. Ogawa, T., R.J. Spina, W.H. Martin, W.M. Kohrt, K.B. Schechtman, J.O. Holloszy, and A.A. Ehsani. Effects of aging, sex and physical training on cardiovascular responses to exercise. Circul. 86:494-503, 1992.

206. Oscai, L.B., B.T. Williams, and B.A. Hertig. Effect of exercise on blood volume. $J$. Appl. Physiol. 24:622-624, 1968.

207. Pate, R.R., P.B. Sparling, G.E. Wilson, K.J. Cureton, and B.J. Miller. Cardiorespiratory and metabolic responses to submaximal and maximal exercise in elite women distance runners. Inter. J. Sports Med. 8:S91-S95, 1987. 
208. Pate, R.R. and A. Kriska. Physiological basis of sex difference in cardiorespiratory endurance. Sports Med. 1:87-98, 1984.

209. Peronett F., R.J. Ferguson, H. Perrault, G. Ricci, and D. Lajoie. Echocardiography and the athlete's heart. Phys. and Sports Med. 9(5):103-112, 1981.

210. Pines A., E.Z. Fisman, Y. Levo, M. Averbuch, A. Lidor, Y. Drory, A. Finkelstein, M. Hetman-Peri, M. Moshlowitz, E. Ben-Ari, and D. Ayalon. The effects of hormone replacement therapy in normal post-menoupausal women: Measurement od Dopplerderived parameters of aortic flow. Am. J. Obstet. Gynecol. 164:806-812, 1991.

211. Plotnick, G.D., L.C. Becker, M.L. Fisher, G. Gerstenblith, D.G. Renlund, J.L. Fleg, M.L. Weisfeldt, And E.G. Lakatta. Use of the Frank-Starling mechanism during submaximal versus maximal upright exercise. Am. J. Physiol. 25:H1101-H1 105, 1986.

212. Poliner, L.R., G.J. Dehmer, S.E. Lewis, R.W. Parkey, C.G. Blomqvist, and J.T. Willerson. Left ventricular performance in normal subjects: A comparison of responses to exercise in the upright and supine positions. Circul. 62(3): 528-534, 1980.

213. Pollak, S.J., S.T. McMillan, E. Mumpower, R. Wharff, W. Knopf, J.M. Felner, and A.P. Yoganathan. Echocardiographic analysis of elite female distance runners. Inter. J. Sports Med. 2:81-83, 1987.

214. Pollock, M.L., C. Foster, D. Knapp, J.L. Rod, and D.H. Schmidt. Effect of age and training on aerobic capacity and body composition of master athletes. J. Appl. Physiol. 62:725-731, 1987.

215. Port, S., F.R. Cobb, R.E. Coleman, and R.H. Jones. Effect of age on the response of the response of the left ventriclar ejection fraction to exercise. N. Engl. J. Med. 303:1133-1137, 1980

216. Powers S.K., D. Martin, and S. Dodd. Exercise-induced hypoxemia in elite endurance athletes: incidence, causes and impact on $\mathrm{VO}_{2} \max$. Sports Med. 16(1):14-22, 1993.

217. Powers S.K., and J. Williams. Exercise-induced hypoxemia in highly trained athletes: a review. Sports Med. 4:46-53,1987.

218. Proctor, D.N., J.R. Halliwill, D.P. Seamans and M.J. Joyner. Active muscle mass and the age-related decline in $\mathrm{VO}_{2}$ max. Med. Sci. Sports Exerc. 28(5):S34, 1996 (abstract). 
219. Renlund, D.G., G. Gerstenblith, R.J. Rodeheffer, J.L. Fleg and E.G. Lakatta. Potency of the Frank-Starling reserve in normal man. J. Am. Coll. Cardiol. 5:514, 1985 (abstract).

220. Rerych, S.K., P.M. Scholz, D.C. Sabiston, and R.H. Jones. Effects of exercise training on left ventricular function in normal subjects: A longitudinal study of radionucleotide angiography. Am. J. Cardiol. 45:224-274, 1980.

221. Riley-Hagen, M.R., R.M. Peshock, J. Sray-Gundersen, J. Katz, R.W. Ryschon, and J.H. Mitchell. Left ventricular dimensions and mass using magnetic resonance imaging in female endurance athletes. Am. J. Cardiol. 69:1067-1074, 1992.

222. Rivera, A.M., A.E. Pels III, S.P. Sady, M.A. Sady, E.M. Cullinane and P.D. Thompson. Physiological factors associated with the lower maximal oxygen consumption in masters runners. J. Appl. Physiol. 66(2):949-954, 1989.

223. Robertson R.J., G. Ronald, K.F. Metz, C.J. Caspersen, T.G. Allison, R.A. Abbott, G.S. Skirnar, J.R. Krause, and P.A. Nixon. Hemoglobin concentration and aerobic work capacity in women following induced erythrocythemia. J. Appl. Physiol. 57(2):568-575, 1984.

224. Rodeheffer, R.J., G. Gerstenblith, L.C. Becker, J.L. Fleg, M.L. Weisfeldt, and E.G. Lakatta. Exercise cardiac output is maintained with advancing age in healthy human subjects: cardiac dilation and increased stroke volume compensate for a diminished heart rate. Circul. 69, 203-213, 1984.

225. Rogers, M.A., and W.J. Evans. Changes in skeletal muscle with aging: Effects of exercise training. In: J.O. Holloszy (Ed.) Exercise and Sport Sciences Reviews, Vol. 21, Baltimore: Williams \& Willkins, 1993, pp. 65-102.

226. Rogers, M.A., J.M. Hagberg, W.H. Martin III, A.A. Ehsani, and J.O. Holloszy. Decline in $\mathrm{VO}_{2} \mathrm{max}$ with aging in master athletes and sedentary men. J. Appl. Physiol. 68(5):2195-2199, 1990.

227. Rost, R. and W. Hollman. Athlete's heart: A review of its historical assessment and new aspects. Int. J. Sports Med. 4:147-164, 1983.

228. Rowell, L.B., Cardiovascular adaptaions to chronic physical activity and inactivity. In: Human Circulation Regulation During Physical Stress. Oxford University Press, 1986, pp. 257-286. 
229. Rubal, B.J., A.R. Al-Muhailani, J. Rosentsweig. Effects of physical conditioning on the heart size and wall thickness of college women. Med. Sci. Sports Exerc. 19:423-429, 1987.

230. Rubal, B.J., J.M. Moody, S. Damore, S.R. Bunker, and N.M. Diaz. Left ventricular performance of the athletic heart during upright exercise: A heart rate controlled study. Med. Sci. Sports Exerc. 18(1):134-140, 1986.

231. Rubal, B.J., J. Rosentsweig, and B. Hammerly. Echocardiographic examination of women collegiate softball players. Med. Sci. Sports Exerc. 13:176-179, 1981.

232. Sabbah. H.N., and P.P. Stein. Pressure-diameter relations dur early diastole in dogs: Incompatibility with the concept of passive left ventricular filling. Circul. Res. 45:357$365,1981$.

233. Saltin, B. and S. Strange. Maximum oxygen uptake: "old" and "new" arguments for a cardiovascular limitation. Med. Sci. Sports Exerc. 24:30-37, 1992.

234. Saltin B. The aging endurance athlete. In: J.R. Sutton, and R.M. Brock (Eds.) Sports Medicine for the Mature Athlete, Indianapolis, Indiana: Benchmark Press, 1986, pp. 59-79.

235. Saltin B., J. Henrickson, E. Nygaard, P. Andersen and E. Jansson. Fibre types and metabolic potentials of skeletal muscle in sedentary man and endurance runners. $A n n$. $N Y$ Acad. Sci. 301:3-29, 1977.

236. Sanghvi V.R., F. Khaja, A.L. Mack, and J.O. Parker. Effects of blood volume expansion on left ventricular hemodynamics in man. Circul. 46:780-787, 1972.

237. Sartori M.P., M.A. Quinones, and L.C. Kuo. Relation of doppler derived left ventricular filling parameters to age and radius/ thickness ratio in normal and pathalogic states. Am. J Cardiol. 59:1179-1182, 1987.

238. Schaible, T.F., S. Penpargkul and J. Scheuer. Cardiac responses to exercise training in male and female rats. J Appl. Physiol. 50:112-117, 1981.

239. Scheuer J., A. Malhotra, T.F. Schaible, and J. Capasso. Effects of gonadectomy and hormone replacement on rat hearts. Circul. Res. 61:12-19, 1987.

240. Scheuer J., and C.M. Tipton. Cardiovascular adaptations to physical training. Ann. Rev. Physiol. 39:221, 1977. 
241. Schocken, D.D., J.A. Blumenthal, S. Port, P. Hindle, and R.E. Coleman. Physical conditioning and left ventricular performance in the elderly: Assessment by radionucleotide angiocardiography. Am. J. Cardiol. 52:359-364, 1983.

242. Schoene R.B., H.T. Robertson, D.J. Pierson and A.P. Peterson. Respiratory drives and exercise in menstrual cycles of athletic and nonathletic women. J. Appl. Physiol. 50:1300-1305, 1981.

243. Schulman, SP., E.G. Lakatta, J.L. Fleg, L.C. Becker, and G. Gerstenblith. Agerelated decline in left ventricular filling at rest and exercise: another manifestation of decreased beta-adrenergic responsiveness in the elderly. Am. J. Physiol. 263:H1932H1938, 1992.

244. Schunkert H., A.H. Jan Dancer, H.W. Hense, H.M. Fans, S. Kurzinger and G.A.J. Riegger. Effect of estrogen replacement therapy on the renin-angiotensin system in postmenopausal women. Cicul. 95:39-45, 1997.

245. Seals, DR., J.M. Hagberg, R.J. Spina, M.A. Rogers, K.B. Schectman, and A.A. Ehsani. Enhanced left ventricular performance in endurance trained older men. Circul. 89:198-205, 1994.

246. Seals, DR., J.M. Hagberg, B.F. Hurley, A.A. Ehsani and J.O. Holloszy. Endurance training in older men and women:I. Cardiovascular responses to exercise. J. Appl. Physiol. 57(4):1024-1029, 1984.

247. Senay, L.C., D. Mitchell, and C.H. Wyhdham. Acclimatization in a hot humid environment: body fluid adjustments. J. Appl. Physiol. 40:786-796, 1976.

248. Shangold M.M., R.W. Rebar, A. Colston, A.C. Wentz, and I. Schiff. Evaluation and management of menstrual dysfunction in athletes. J. Am. Med. Assoc. 263:1665$1669,1990$.

249. Shangold M.M., R. Freeman, B. Thysen and M. Gatz. The relation between longdistance running, plasma progesterone and luteal phase length. Fertil. Steril. 31:130, 1979.

250. Shapiro, L.M. and R.G. Smith. Effects of training on left ventricular structure and function: An echocardiographic study. Br. Heart J. 50:534-539, 1983.

251. Shaw, D.J., D.A. Rithman, C.S. Angell, and N.W. Shock. The effects of age and blood pressure upon the systolic time intervals in males aged 20-89 years. J. Gerontol. 
28:133-139, 1973.

252. Shephard, RJ. and K.H. Sidney. Exercise and aging. Exerc. Sports Sci. Rev. 6:1-57, 1978.

253. Simineau, J.A., G. Lortie, M.R. Boulay, M.C. Thibault, G. Theriault and C. Bouchard. Skeletal muscle histochemical characteristics in sedentary males and female subjects. J. Clin. Pharmacol. 63:30-35, 1985.

254. Smyth, R.J., N. Gledhill, A.B. Froese, and V. Jamnik. Validation of non-invasive maximal cardiac output measurement. Med. Sci. Sports Exerc. 16:512-515, 1984.

255. Sparling, P.B. A meta-analysis of studies comparing maximum oxygen uptake in men and women. Res. Quart. Exerc. Sport (51):542-552, 1980.

256. Spina, R.J., T.R. Miller, W.H. Bogenhagen, K.B. Schechtman and A.A. Ehsani. Gender-related differences in left ventricular filling dynamics in older subjects after endurance trianing. J. Gerontol. 51A(3):B232-237, 1996.

257. Spina, R.J., T. Ogawa, W.H. Martin III, A.R. Coggan, J.O. Holloszy, and A.A. Ehsani. Differences in cardiovascular adaptations to endurance exercise training between older men and women. J. Appl. Physiol. 75(2):849-855, 1993a.

258. Spina, R.J., T. Ogawa, T.R. Miller, W.M. Kohrt, and A.A. Ehsani. Effects of exercise training on left ventricular performance in older females free of cardiopulmonary disease. Am. J. Cardiol. 71:99-104, 1993b.

259. Spina, R.J., T. Ogawa, W.H. Martin III, A.A. Ehsani. Exercise training prevents decline in stroke volume during exercise in young healthy subjects. J. Appl. Physiol. 72(6):2458-2462, $1992 \mathrm{a}$.

260. Spina, R.J., T. Ogawa, A.R. Coggan. J.O. Holloszy and A.A. Ehsani. Exercise training improves left ventricular contractile response to $\beta$-adrenergic agonist. J. Appl. Physiol. 72(6):307-311, 1992b.

261. Spirito P., and B.J. Maron. Influence of aging on Doppler echocardiographic indicies of left ventricular diastolic function. Br. Heart J. 59:672-679, 1988.

262. Spurgeon, HA., M.F. Steinbach, and E.G. Lakatta. Chronic exercise prevents characteristic age-related changes in rat cardiac contraction. Am. J. Phsiol. 
244(13):H513-H518, 1983.

263. Stephensen L.A., M.A. Kolka, R. Francesconi, and R.R. Gondalez. Circadian variation in plasma renin activity, catecholamines and aldosterone during exercise in women. Eur. J. Appl. Physiol. 58:756-764, 1989.

264. Stephensen L.A., and M.A. Kolka. Plasma volume during heat stress and exercise in females. Eur. J. Appl. Physiol. 57:373-381, 1988.

265. Stevenson, E.T., K.P. Davy, M.J. Reiling, and and D.R. Seals. Maximal aerobic capacity and total blood volume in highly trained middle-aged and older female endurance athletes. J. Appl. Physiol. 77(4):1691-1696, 1994.

266. Stratton, J.R., M.D. Cerqueira, R.S. Schwartz, W.C. Levy, R.C. Veith, S.E. Kahn and I.B. Arass. Differences in cardiovascular response to isoproterenol in relation to age and exercise training in healthy males. Circul. 86:504-512, 1992.

267. Strandell, T. Circulatory studies in healthy old men. Acta. Med. Scand. 175:1-43, 1964.

268. Sullivan, M.J., F.R. Cobb, and M.B. Higginbotham. Stroke volume increases by similar mechanisms during upright exercise in normal men and women. Am. J. Cardiol. 67:1405-1412, 1991.

269. Taffet G.E. and C.A. Tate. CaATPase content is lower in cardiac sarcoplasmic reticulum isolated from old rats. Am. J. Physiol. 264(33):H1609-H1614, 1993.

270. Takemoto, K.A., L. Bernstein, J.F. Lopez, D. Marshak, S.H. Rahimtoola, and P.A.N. Chandraratna. Abnormalities of diastolic filling of the left ventricle associated with aging are less pronounced in exercise-trained individuals. Am. Heart J. 124:143-148, 1992.

271. Tankersley, C.G., W.C. Nichols, D.R. Deaver, D. Mitka and W.L. Kenney. Estrogen replacement in middle-aged women: Thermoregulatory, cardiovascular and body fluid resposnes to exercise in the heat. J. Appl. Physiol. 73:1238-1245, 1992.

272. Tate, C.A., M.F. Hyek, and G.E. Taffet. Mechanisms for the responses of cardiac muscle to physical activity in old age. Med. Sci. Sports Exerc. 26(5):561-567, 1994.

273. Tate, C.A., G.E. Taffet, E.K. Hudson, S.L. Blaylock, R.P. McBride and L.H. Michael. Enhanced calcium uptake of cardiac sarcoplasmic reticulum in exercise trained 
old rats. Am. J. Physiol. 258:H431-H435, 1990.

274. Thoden, J.S. Testing aerobic power. In: D.MacDougall, et al. (Eds.) Physiological Testing of the High Performenace Athlete, Champaign, Il: Human Kinetics, 1990, pp. 107-173.

275. Thomas, S.G., D.H. Paterson, D.A. Cunningham, D.G. McLellan, and W.J. Kostuk. Cardiac output and left ventricular function in response to exercise in older males. Can.J. Clin. Pharmacol. 71:136-144, 1993.

276. Trappe, S.W., D.L. Costill, M.D. Vukovick, J.Jones, T. Melham. Aging among elite endurance distance runners: a 22 yr longitudinal study. J. Appl. Physiol. 80(1):285-290, 1996.

277. Turner, C., and S. Fortney. Daily plasma volume changes during the menstrual cycle. Fed. Proc. 43:718, 1984 (abstract).

278. Udelson J.E., S.L. Bacharach, R.O. Cannon III, and R.O. Bonow. Minimal left ventricular pressure during $\beta$-adrenergic stimulation in human subjects. Evidence for elastic recoil and diastolic "suction" in the normal heart. Circul. 82:1174-1182, 1990.

279. Underwood, R.H., and J.L. Schwade. Noninvasive analysis of cardiac function of elite endurance runners-echocardiography, vectorography and cardiac intervals. Ann. N. Y. Acad. Sci. 301:297-309, 1977.

280. Upton, S.J., R.D. Hagen, B. Lease, J. Rosentsweig, L.R. Gettman, and J.L. Duncan. Comparitive physiological profiles among young and middle-aged female distance runners. Med. Sci. Sports Exerc. 16:67-71, 1984.

281. Upton, S.J., R.D. Hagan, J. Rosentsweig, and L.R. Gettman. Comparison of the physiological profiles of middle-aged women distance runners and sedentary women. Res. Quart. Exerc. Sport 54:83-87, 1983.

282. Vaccaro, P. Body composition and physiological responses of masters female swimmers, 20- to 70 years of age. Res. Quart. Exerc. Sport. 55:278-284, 1984.

283. Vaccaro, P., G.M. Dummer, and D.H. Clarke. Physiological characteristics of female masters swimmers. Phys. Sports Med. 9(12):75-78, 1981.

284. Vaitkevicius, P.V., J.L. Fleg, J.H. Engel, F.C. O'Connor, J.G. Wright, L.E. Lakatta, F.C.P. Yin and E.G. Lakatta. Effect of age on aerobic capacity on arterial stiffness in 
healthy adults. Circul. 88:1456-1462, 1993.

285. Vanfraechem, J.H.P. Stroke volume and systolic time interval adjustments during bicycle exercise. J. Appl. Physiol. 46:588-592, 1979.

286. Vavra, J., J. Sova, and M. Macek. Effect of age on systolic time intervals at rest and during exercise on a bicycle ergometer. Eur. J. Appl. Physiol. 50:71-78, 1982.

287. Vellar, O.D. Changes in haemoglobin and haematocrit during the menstrual cycle. Acta. Obstet. Gynecol. Scand. 53: 243-246, 1974.

288. Wanderman, K.L., Z. Hayek, I. Ovsyscher, G. Loutaty, A. Cantor, Y. Gussarsky, and $M$. Gueron. Systolic time intervals in adolescents: normal standards for clinical use and comparison with children and adults. Circul. 63(1):204-209, 1981.

289. Warburton, D.E.R. "Effect of acute plasma volume expansion on the endurance performance, $\mathrm{VO}_{2} \mathrm{max}$, and cardiac function in endurance trained athletes." (Thesis) North York: Graduate Program in Exercise and Health Science, Sept. 1996.

290. Warren, BJ., D.C. Nieman, R.G. Dotson, C.H. Adkins, K.A. O'Donnell, B.L. Haddock, and D.E. Butterworth. Cardiorespiratory responses to exercise training in Septuagenarian women. Int. J. Sports Med. 14(2):60-65, 1993.

291. Weissler, A.M. Current concepts in cardiology: systolic time intervals. New. Engl. J. Med. 296(6): 321-324, 1977.

292. Weissler, A.M., W.S. Harris, and C.D. Schoenfeld. Systolic time intervals in heart failure in man. Circul. 37:149-159, 1968.

293. Weissler, A.M., R.G. Peeler, and W.H. Roehll, Jr. Relationships between left ventricular ejection time, stroke volume and heart rate in normal individuals and patients with cardiovascular disease. Am. Heart J. 62:367-378, 1961 a.

294. Weissler, A.M., B.K. Kolmen, W.H. Roehll, and R.G. Peeler. Influence of age and sex on ventricular ejection dynamics in man. Circul. 24:1068, 1961b (abstract).

295. Wells, CL., M.A. Boorman and D.M. Riggs. Effect of age and menopausal status on cardio-respiratory fitness in masters women runners. Med. Sci. Sports Exerc. 24(10):1147-1154, 1992. 
296. Wells, C.L. and S.A. Plowman. Sexual differences in athletic performance: Biological or behavioural? Phys. and Sports Med., 11(8): 52-63, 1983.

297. Wells, C.L., J. Stern and L. Hecht. Haematological changes following a marathon race in male and females runners. Eur. J. Appl. Physiol. 48:41-49, 1981.

298. Wells, C.L. and S.M. Horvath. Heat stress responses relative to the menstrual cycle. J. Appl. Physiol. 35(1):1-5, 1973.

299. West, J.B. Respiratory Physiology - The Essentials (5th Ed.) Baltimore, U.S.A.: Willaims and Williams, 1995, pp. 51-70.

300. Wilkerson, J.E., E.M. Leeds and G.D. Brown. Hematological differences in regularly cycling females with normal or low luteal progesterone. Fed. Proc. 44:486, 1985 (abstract).

301. Willems, Л., J. Roelandt, H. De Geest, H. Kesteloot and J. V. Jossens. The left ventricular ejection time in elderly subjects. Circul. 42:37-42, 1970.

302. Willems, J. and H. Kesteloot. The left venricular ejection time: It's relationship to heart rate, mechanical systole and some anthropometric data. Acta. Cardiol. 22:401, 1967.

303. Williams, J.K., M.R. Adams, H.S. Klopfenstein. Estrogen modulates responses of athlerosclerotic coronary arteries. Circul. 81:1680-1687, 1990.

304. Wilmore, J.H., and D.L. Costill. Physiology of Sport and Exercise, Champaign, Ill: Human Kinetics, 1994, pp. 162-189.

305. Wilmore J.H. The application of science to sports: Physiological profiles of males and females athletes. Can. J. Applied Sports Sci., 4:103-115, 1979.

306. Wilmore, J.H. and C.H. Brown. Physiological profiles of women distance runners. Med. Sci. Sports Exerc. 6:178-181, 1974.

307. Wolfe, L.A., A. Laprade, G.W. Burggraf, and R. Norman. Cardiac responses of young women to conditioning for a 10 kilometer race. Int. J. Sports Med. 13:384-389, 1992.

308. Wolfe, L.A., R.P. Martin, D.D. Watson, R.D. Lasley, and D.E. Burns. Chronic exercise and left ventricular structure and function in healthy human subjects. J. Appl. 
Physiol. 58(2):409-415, 1985.

309. Wolfe, L.A. and D.A. Cunningham. Effects of chronic exercise on cardiac output and its determinants. Can. J. Physiol. Pharmacol. 60: 1089-1097, 1982.

310. Wolfe, L.A., D.A. Cunningham, P.A. Bechnitzer, and P.H. Nichol Effects of endurance training on left ventricular dimensions in healthy men. J. Appl. Physiol. 47(1):207-212, 1979.

311. Wolfe, L.A., D.A. Cunningham, G.M. Davis, and H. Rosenfeld. Relationship between maximum oxygen uptake and left ventricular function in exercise. J. Appl. Physiol. 44:44-49, 1978.

312. Woodiwiss, A.J. and G.R. Norton. Exercise-induced cardiac hypertrophy is associated with an increased myocardial compliance. J. Appl. Physiol. 78(4):1303-1311, 1995.

313. Wyndham, C. H., A.J.A. Benade, C.G. Williams, N.I. Strydom, A. Goldin and A.J.A. Heynes. Changes in central circulation and body fluid spaces during acclimatization to heat. J. Appl. Physiol. 25:586-593, 1968.

314. Wyndham, C.H., J.F. Morrison, and C.G. Williams. Heat reactions of male and female Caucasions. J. Appl. Physiol. 20(3):357-364, 1965.

315. Yellin, E.L., S. Nikolic, and R.W.M. Frater. Left ventricular filling dynamics and diastolic function. Prog. Cardiovasc. Dis. 32(4):247-271, 1990.

316. Yin, F.C.P., M.L. Weisfeldt, W.R. Milnor. Role of aortic input impedence in the decerased cardiovascular response to exercise with aging in dogs. J. Clin. Invest. 68:28$38,1981$.

317. Yuhasz, M.S. Physical Fitness and Sports Appraosal Laboratory Manual, London, The University of Western Ontario, 1966.

318. Younis, L.T., J.A. Melin, A.R. Roberts, and M.R. Detry. Influence of age and sex on left ventricular volumes and ejection fraction during upright exercise in normal subjects. Eur. Heart J. 11:916-924, 1990.

319. Zeldis, S.M., J. Morganroth, and S. Rubler. Cardiac hypertrophy in response to dynamic conditioning in female athletes. J. Appl. Physiol. 44:849-852, 1978. 
320. Zoneraich, S., O. Zoneraich, J.J. Rhee, D. Jordan, and J. Appel. Evaluating the endurance athlete's heart: a non-invasive graphic study. Circul. 52:455-458, 1975.

321. Zwiren, L.D., K.J. Cureton and P. Hutchinson. Comparison of circulatory responses to submaximal exercise in equally trained men and women. Int. J. Sports Med. 4:255-259, 1983. 


\section{APPENDIX A}

RAW DATA

\section{LIST OF TABLES}

TABLE

PAGE

$1 \quad$ Participants' Characteristics $\ldots \ldots \ldots \ldots \ldots \ldots \ldots \ldots \ldots \ldots \ldots \ldots \ldots$

2 Participants' Anthropometric Measurements .............. 218

3 Hematology and Blood Volume ................... 219

$4 \quad$ Oxygen Consumption $\left(\mathrm{ml} \cdot \mathrm{kg}^{-1} \cdot \min ^{-1}\right) \ldots \ldots \ldots \ldots \ldots \ldots \ldots$

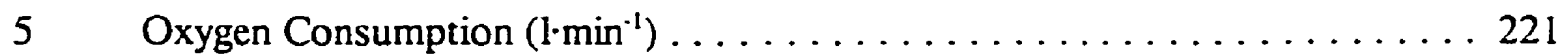

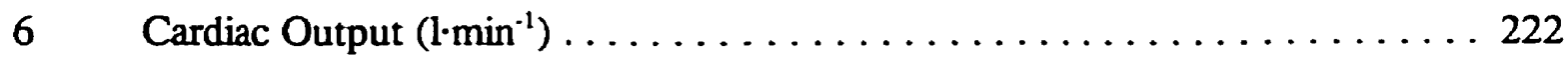

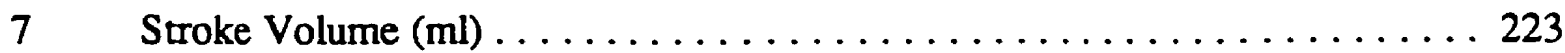

8 Arterial Mixed - Venous Oxygen Difference $\left(\mathrm{ml} \mathrm{O}_{2} \cdot \mathrm{dl}\right.$ of bood $\left.{ }^{-1}\right) \ldots \ldots 24$

$9 \quad$ Left Ventricular Ejection Time $(\mathrm{msec}) \ldots \ldots \ldots \ldots \ldots \ldots \ldots \ldots$

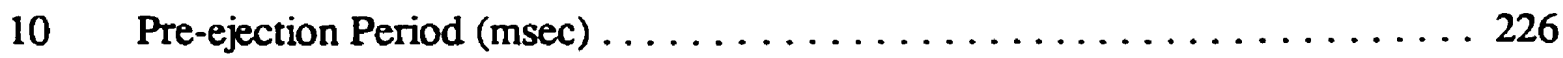

11 Diastolic Filling Time $(\mathrm{msec}) \ldots \ldots \ldots \ldots \ldots \ldots \ldots \ldots \ldots \ldots \ldots \ldots$

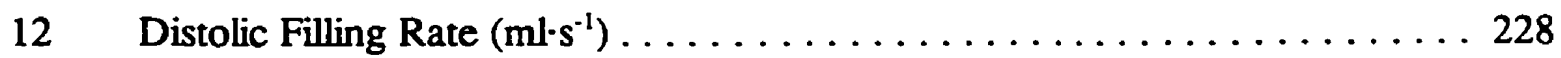

13 Left Ventricular Ejection Rate $\left(\mathrm{ml}^{-1} \mathrm{~s}^{-1}\right) \ldots \ldots \ldots \ldots \ldots \ldots \ldots$

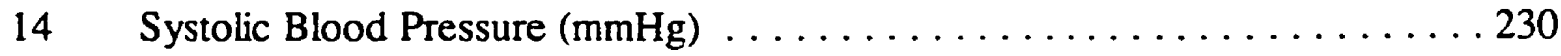

15 Diastolic Blood Pressure $(\mathrm{mmHg}) \ldots \ldots \ldots \ldots \ldots \ldots \ldots \ldots \ldots \ldots$

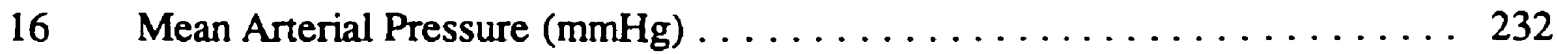

17 Total Peripheral Resistance $\left(m \operatorname{mHg}^{-1} \cdot \min ^{-1}\right) \ldots \ldots \ldots \ldots \ldots \ldots$ 
TABLE 1. Participants' Characteristics

\begin{tabular}{|c|c|c|c|c|c|c|}
\hline Participant & Group & $\begin{array}{l}\text { Age } \\
\text { (yr) }\end{array}$ & $\begin{array}{c}\text { HRmax } \\
\text { (bpm) }\end{array}$ & $\begin{array}{l}\text { HRrest } \\
\text { (bpm) }\end{array}$ & $\begin{array}{l}\text { VC } \\
\text { (l) }\end{array}$ & $\begin{array}{c}\mathrm{VO}_{2} \max \\
\left(\mathrm{ml} \cdot \mathrm{kg}^{-1} \cdot \mathrm{min}^{-1}\right)\end{array}$ \\
\hline $\begin{array}{l}1 \\
2 \\
3 \\
4 \\
5 \\
6 \\
\end{array}$ & $\begin{array}{l}20 \\
20 \\
20 \\
20 \\
20 \\
20 \\
\end{array}$ & $\begin{array}{l}20.5 \\
20.7 \\
21.9 \\
19.7 \\
29.0 \\
22.5 \\
\end{array}$ & $\begin{array}{l}185 \\
192 \\
192 \\
195 \\
194 \\
191 \\
\end{array}$ & $\begin{array}{l}52 \\
58 \\
59 \\
65 \\
77 \\
48 \\
\end{array}$ & $\begin{array}{l}4.64 \\
4.60 \\
4.19 \\
4.38 \\
2.93 \\
3.35 \\
\end{array}$ & $\begin{array}{l}55.0 \\
61.1 \\
61.2 \\
70.0 \\
67.4 \\
67.6 \\
\end{array}$ \\
\hline & $\begin{array}{c}\overline{\mathbf{x}} \\
\pm \text { tsem }\end{array}$ & $\begin{array}{c}22.4 \\
1.4\end{array}$ & $\begin{array}{c}192 \\
1 \\
\end{array}$ & $\begin{array}{c}60 \\
4 \\
\end{array}$ & $\begin{array}{l}4.02 \\
0.29 \\
\end{array}$ & $\begin{array}{r}63.7 \\
2.3 \\
\end{array}$ \\
\hline $\begin{array}{c}7 \\
8 \\
9 \\
10 \\
11 \\
12\end{array}$ & $\begin{array}{l}40 \\
40 \\
40 \\
40 \\
40 \\
40\end{array}$ & $\begin{array}{l}45.0 \\
43.0 \\
40.9 \\
40.8 \\
43.5 \\
43.0\end{array}$ & $\begin{array}{l}183 \\
182 \\
180 \\
180 \\
188 \\
180\end{array}$ & $\begin{array}{l}55 \\
62 \\
59 \\
60 \\
63 \\
62\end{array}$ & $\begin{array}{l}4.35 \\
3.49 \\
3.66 \\
4.74 \\
4.44 \\
3.46\end{array}$ & $\begin{array}{l}44.6 \\
58.9 \\
51.2 \\
44.8 \\
63.2 \\
56.5\end{array}$ \\
\hline & $\begin{array}{c}\overline{\mathbf{x}} \\
\text { 土sem }\end{array}$ & $\begin{array}{c}42.7 \\
0.6 \\
\end{array}$ & $\begin{array}{c}182 \\
1 \\
\end{array}$ & $\begin{array}{c}60 \\
1 \\
\end{array}$ & $\begin{array}{l}4.02 \\
0.22 \\
\end{array}$ & $\begin{array}{c}51.0 \\
3.1 \\
\end{array}$ \\
\hline $\begin{array}{l}13 \\
14 \\
15 \\
16 \\
17 \\
18\end{array}$ & $\begin{array}{l}50 \\
50 \\
50 \\
50 \\
50 \\
50\end{array}$ & $\begin{array}{l}50.8 \\
48.5 \\
49.3 \\
53.8 \\
50.2 \\
52.1\end{array}$ & $\begin{array}{l}173 \\
176 \\
167 \\
163 \\
173 \\
150\end{array}$ & $\begin{array}{l}57 \\
53 \\
57 \\
53 \\
53 \\
53\end{array}$ & $\begin{array}{l}4.08 \\
3.66 \\
3.31 \\
3.92 \\
3.18 \\
3.16\end{array}$ & $\begin{array}{l}44.7 \\
51.6 \\
46.6 \\
57.0 \\
41.2 \\
41.1\end{array}$ \\
\hline & $\begin{array}{c}\overline{\mathbf{x}} \\
\text { 土sem }\end{array}$ & $\begin{array}{c}50.8 \\
0.8\end{array}$ & $\begin{array}{c}167 \\
4\end{array}$ & $\begin{array}{c}54 \\
1\end{array}$ & $\begin{array}{l}3.55 \\
0.16\end{array}$ & $\begin{array}{c}47.0 \\
2.5\end{array}$ \\
\hline $\begin{array}{l}19 \\
20 \\
21 \\
22 \\
23\end{array}$ & $\begin{array}{l}60 \\
60 \\
60 \\
60 \\
60\end{array}$ & $\begin{array}{l}57.7 \\
62.7 \\
62.5 \\
57.3 \\
61.2\end{array}$ & $\begin{array}{l}169 \\
161 \\
151 \\
151 \\
152\end{array}$ & $\begin{array}{l}64 \\
69 \\
51 \\
67 \\
62\end{array}$ & $\begin{array}{l}2.50 \\
2.99 \\
3.28 \\
3.99 \\
3.12\end{array}$ & $\begin{array}{l}40.1 \\
40.7 \\
44.8 \\
45.8 \\
49.4\end{array}$ \\
\hline & $\begin{array}{c}\bar{x} \\
\pm \operatorname{sem}\end{array}$ & $\begin{array}{c}60.3 \\
1.2\end{array}$ & $\begin{array}{c}157 \\
4\end{array}$ & $\begin{array}{c}63 \\
3\end{array}$ & $\begin{array}{l}3.18 \\
0.24\end{array}$ & $\begin{array}{c}44.2 \\
1.7\end{array}$ \\
\hline
\end{tabular}


TABLE 2. Participants' Anthropometric Measurements

\begin{tabular}{|c|c|c|c|c|c|c|}
\hline Participant & Group & $\begin{array}{c}\text { Sum of } 9 \\
\text { Skinfolds } \\
\quad(\mathrm{mm})\end{array}$ & $\begin{array}{c}\text { Body Fat } \\
(\%)^{*}\end{array}$ & $\begin{array}{c}\text { Body } \\
\text { Mass } \\
\text { (kg) }\end{array}$ & $\begin{array}{c}\text { LBM } \\
(\mathrm{kg})\end{array}$ & $\begin{array}{c}\text { Height } \\
\text { (cm) }\end{array}$ \\
\hline $\begin{array}{l}1 \\
2 \\
3 \\
4 \\
5 \\
6\end{array}$ & $\begin{array}{l}20 \\
20 \\
20 \\
20 \\
20 \\
20\end{array}$ & $\begin{array}{l}84.8 \\
58.6 \\
70.6 \\
55.0 \\
71.0 \\
76.7\end{array}$ & $\begin{array}{l}17.5 \\
13.0 \\
15.3 \\
12.5 \\
15.8 \\
16.4\end{array}$ & $\begin{array}{l}65.2 \\
65.0 \\
53.0 \\
58.0 \\
61.8 \\
59.0\end{array}$ & $\begin{array}{l}53.8 \\
56.6 \\
44.9 \\
50.8 \\
52.0 \\
49.3\end{array}$ & $\begin{array}{l}162.5 \\
172.0 \\
165.0 \\
175.0 \\
165.0 \\
159.4\end{array}$ \\
\hline & $\begin{array}{c}\overline{\mathbf{x}} \\
\pm \operatorname{sem}\end{array}$ & $\begin{array}{c}69.4 \\
4.5 \\
\end{array}$ & $\begin{array}{l}15.1 \\
0.8 \\
\end{array}$ & $\begin{array}{c}60.3 \\
1.9\end{array}$ & $\begin{array}{c}51.2 \\
1.9\end{array}$ & $\begin{array}{c}166.5 \\
2.4\end{array}$ \\
\hline $\begin{array}{c}7 \\
8 \\
9 \\
10 \\
11 \\
12\end{array}$ & $\begin{array}{l}40 \\
40 \\
40 \\
40 \\
40 \\
40\end{array}$ & $\begin{array}{c}98.2 \\
68.6 \\
76.0 \\
65.7 \\
69.3 \\
105.9\end{array}$ & $\begin{array}{l}20.8 \\
16.2 \\
17.2 \\
15.7 \\
16.1 \\
21.9\end{array}$ & $\begin{array}{l}63.8 \\
58.9 \\
66.4 \\
63.3 \\
64.6 \\
58.5\end{array}$ & $\begin{array}{l}50.5 \\
49.4 \\
55.0 \\
53.4 \\
54.2 \\
45.7\end{array}$ & $\begin{array}{l}157.5 \\
157.5 \\
171.0 \\
166.0 \\
172.0 \\
164.0\end{array}$ \\
\hline & $\begin{array}{c}\overline{\mathbf{x}} \\
\pm \text { sem }\end{array}$ & $\begin{array}{c}80.6 \\
7.0\end{array}$ & $\begin{array}{c}18.0 \\
1.1\end{array}$ & $\begin{array}{c}62.6 \\
1.3\end{array}$ & $\begin{array}{c}51.4 \\
1.4\end{array}$ & $\begin{array}{c}164.7 \\
2.6\end{array}$ \\
\hline $\begin{array}{l}13 \\
14 \\
15 \\
16 \\
17 \\
18\end{array}$ & $\begin{array}{l}50 \\
50 \\
50 \\
50 \\
50 \\
50\end{array}$ & $\begin{array}{c}76.7 \\
90.6 \\
59.2 \\
60.8 \\
126.8 \\
75.8\end{array}$ & $\begin{array}{l}18.0 \\
19.8 \\
14.9 \\
15.4 \\
25.2 \\
17.7\end{array}$ & $\begin{array}{l}60.0 \\
55.8 \\
67.0 \\
58.1 \\
67.8 \\
57.0\end{array}$ & $\begin{array}{l}49.2 \\
44.8 \\
57.0 \\
49.2 \\
50.7 \\
46.9\end{array}$ & $\begin{array}{l}170.0 \\
158.1 \\
185.0 \\
165.0 \\
168.0 \\
162.0\end{array}$ \\
\hline & $\begin{array}{c}\overline{\mathbf{x}} \\
\pm \text { sem }\end{array}$ & $\begin{array}{l}81.6 \\
10.2 \\
\end{array}$ & $\begin{array}{c}18.5 \\
1.5 \\
\end{array}$ & $\begin{array}{c}61.0 \\
2.1 \\
\end{array}$ & $\begin{array}{c}49.6 \\
1.7 \\
\end{array}$ & $\begin{array}{c}168.0 \\
3.8 \\
\end{array}$ \\
\hline $\begin{array}{l}19 \\
20 \\
21 \\
22 \\
23\end{array}$ & $\begin{array}{l}60 \\
60 \\
60 \\
60 \\
60\end{array}$ & $\begin{array}{l}80.6 \\
93.9 \\
71.4 \\
79.6 \\
64.8\end{array}$ & $\begin{array}{l}18.8 \\
21.1 \\
17.7 \\
18.6 \\
16.5\end{array}$ & $\begin{array}{l}58.6 \\
54.3 \\
51.4 \\
67.1 \\
53.2\end{array}$ & $\begin{array}{l}47.6 \\
42.8 \\
42.3 \\
54.6 \\
44.4\end{array}$ & $\begin{array}{l}165.1 \\
158.8 \\
158.0 \\
168.9 \\
168.9\end{array}$ \\
\hline & $\begin{array}{c}\overline{\mathbf{x}} \\
\pm \text { sem }\end{array}$ & $\begin{array}{c}78.1 \\
4.9\end{array}$ & $\begin{array}{c}18.5 \\
0.8\end{array}$ & $\begin{array}{c}56.9 \\
2.8\end{array}$ & $\begin{array}{c}46.3 \\
2.3\end{array}$ & $\begin{array}{c}163.9 \\
2.4\end{array}$ \\
\hline
\end{tabular}

* Jackson \& Pollock sum of 7 skinfolds (Appendix E and refererence 134) 
TABLE 3. Haematology and Blood Volume

\begin{tabular}{|c|c|c|c|c|c|c|}
\hline Participant & Group & $\begin{array}{l}\text { BV } \\
\text { (ml) }\end{array}$ & $\begin{array}{c}\text { BV } \\
\left(\mathrm{ml} \cdot \mathrm{kg}^{-1}\right)\end{array}$ & $\begin{array}{c}\text { BV } \\
(\mathrm{ml} \cdot \mathbf{k g} \\
\left.\mathrm{lbm}^{-1}\right)\end{array}$ & $\begin{array}{l}\text { [Hb] } \\
(\mathrm{g} \%)\end{array}$ & $\begin{array}{l}\text { Hct } \\
(\%)\end{array}$ \\
\hline $\begin{array}{l}1 \\
2 \\
3 \\
4 \\
5 \\
6\end{array}$ & $\begin{array}{l}20 \\
20 \\
20 \\
20 \\
20 \\
20\end{array}$ & $\begin{array}{l}5485 \\
5270 \\
4650 \\
4733 \\
5439 \\
4656\end{array}$ & $\begin{array}{l}84.1 \\
81.1 \\
87.7 \\
81.6 \\
88.0 \\
78.9\end{array}$ & $\begin{array}{c}102.0 \\
93.1 \\
103.6 \\
93.2 \\
104.6 \\
94.4\end{array}$ & $\begin{array}{l}14.1 \\
14.8 \\
14.1 \\
13.8 \\
15.6 \\
13.8\end{array}$ & $\begin{array}{l}39.5 \\
42.8 \\
39.5 \\
41.6 \\
48.0 \\
37.5\end{array}$ \\
\hline & $\begin{array}{c}\bar{x} \\
\pm \text { sem }\end{array}$ & $\begin{array}{c}5039 \\
164\end{array}$ & $\begin{array}{c}83.6 \\
1.5\end{array}$ & $\begin{array}{c}98.5 \\
2.2\end{array}$ & $\begin{array}{l}14.4 \\
0.3\end{array}$ & $\begin{array}{r}41.5 \\
1.5\end{array}$ \\
\hline $\begin{array}{c}7 \\
8 \\
9 \\
10 \\
11 \\
12\end{array}$ & $\begin{array}{l}40 \\
40 \\
40 \\
40 \\
40 \\
40\end{array}$ & $\begin{array}{l}4373 \\
4157 \\
5018 \\
5580 \\
5586 \\
4160\end{array}$ & $\begin{array}{l}68.5 \\
70.6 \\
75.6 \\
88.2 \\
86.5 \\
71.1\end{array}$ & $\begin{array}{c}86.6 \\
84.2 \\
91.2 \\
104.5 \\
103.1 \\
91.0\end{array}$ & $\begin{array}{l}13.0 \\
14.5 \\
13.5 \\
13.6 \\
14.9 \\
14.2\end{array}$ & $\begin{array}{l}36.5 \\
43.0 \\
39.2 \\
39.5 \\
41.8 \\
41.4\end{array}$ \\
\hline & $\begin{array}{c}\bar{x} \\
\pm \text { sem } \\
\end{array}$ & $\begin{array}{c}4812 \\
276\end{array}$ & $\begin{array}{c}76.8 \\
3.5 \\
\end{array}$ & $\begin{array}{c}93.4 \\
3.4\end{array}$ & $\begin{array}{c}14.0 \\
0.3\end{array}$ & $\begin{array}{c}40.2 \\
0.9\end{array}$ \\
\hline $\begin{array}{l}13 \\
14 \\
15 \\
16 \\
17 \\
18\end{array}$ & $\begin{array}{l}50 \\
50 \\
50 \\
50 \\
50 \\
50\end{array}$ & $\begin{array}{l}4639 \\
4070 \\
6265 \\
6244 \\
4791 \\
5786\end{array}$ & $\begin{array}{c}77.3 \\
72.9 \\
93.5 \\
107.5 \\
70.7 \\
101.5\end{array}$ & $\begin{array}{c}94.3 \\
90.8 \\
109.9 \\
126.9 \\
94.5 \\
123.4\end{array}$ & $\begin{array}{l}15.0 \\
15.5 \\
16.2 \\
13.6 \\
14.1 \\
13.7\end{array}$ & $\begin{array}{l}42.8 \\
44.7 \\
42.4 \\
42.6 \\
42.2 \\
39.7\end{array}$ \\
\hline & $\begin{array}{c}\overline{\mathbf{x}} \\
\pm \text { sem }\end{array}$ & $\begin{array}{c}5299 \\
377\end{array}$ & $\begin{array}{c}87.2 \\
6.4\end{array}$ & $\begin{array}{c}106.6 \\
6.5\end{array}$ & $\begin{array}{c}14.7 \\
0.4\end{array}$ & $\begin{array}{r}42.4 \\
0.6 \\
\end{array}$ \\
\hline $\begin{array}{l}19 \\
20 \\
21 \\
22 \\
23\end{array}$ & $\begin{array}{l}60 \\
60 \\
60 \\
60 \\
60\end{array}$ & $\begin{array}{l}5712 \\
3870 \\
4090 \\
5282 \\
3805\end{array}$ & $\begin{array}{l}97.5 \\
71.3 \\
79.6 \\
78.7 \\
71.5\end{array}$ & $\begin{array}{c}120.0 \\
90.4 \\
96.7 \\
96.7 \\
85.7\end{array}$ & $\begin{array}{l}14.5 \\
14.9 \\
14.3 \\
13.6 \\
14.7\end{array}$ & $\begin{array}{l}41.0 \\
41.6 \\
40.9 \\
41.0 \\
44.0\end{array}$ \\
\hline & $\begin{array}{c}\bar{x} \\
\text { tsem }\end{array}$ & $\begin{array}{c}4552 \\
395\end{array}$ & $\begin{array}{c}79.7 \\
4.8\end{array}$ & $\begin{array}{c}97.9 \\
5.9\end{array}$ & $\begin{array}{c}14.4 \\
0.2\end{array}$ & $\begin{array}{c}41.7 \\
0.6\end{array}$ \\
\hline
\end{tabular}


TABLE 4. Oxygen Consumption $\left(\mathrm{ml} \cdot \mathrm{kg}^{-1} \cdot \mathrm{min}^{-1}\right)$

\begin{tabular}{|c|c|c|c|c|c|c|c|}
\hline \multirow[t]{2}{*}{ Participant } & \multirow[t]{2}{*}{ Group } & \multicolumn{6}{|c|}{ HR } \\
\hline & & Rest & 110 & 130 & 150 & 170 & $\operatorname{Max}$ \\
\hline 1 & 20 & 5.78 & 18.6 & 26.0 & 31.7 & 46.2 & 55.0 \\
\hline 2 & 20 & 5.07 & 26.2 & 29.8 & 37.0 & 44.7 & 61.1 \\
\hline 3 & 20 & 4.50 & 20.6 & 28.7 & 38.7 & 47.2 & 61.2 \\
\hline 4 & 20 & 2.74 & 20.8 & 30.1 & 46.3 & 54.6 & 70.0 \\
\hline 5 & 20 & 3.25 & 19.0 & 24.6 & 33.4 & 39.4 & 67.4 \\
\hline \multirow[t]{2}{*}{6} & 20 & 4.56 & 28.7 & 30.2 & 40.6 & 47.7 & 67.6 \\
\hline & $\begin{array}{c}\overline{\mathbf{x}} \\
\pm \text { sem }\end{array}$ & $\begin{array}{l}4.32 \\
0.46\end{array}$ & $\begin{array}{c}22.3 \\
1.7\end{array}$ & $\begin{array}{c}28.2 \\
1.0\end{array}$ & $\begin{array}{c}38.0 \\
2.1\end{array}$ & $\begin{array}{c}46.6 \\
2.0\end{array}$ & $\begin{array}{c}63.7 \\
2.3\end{array}$ \\
\hline 7 & 40 & 4.68 & 18.1 & 25.9 & 32.4 & 41.7 & 44.6 \\
\hline 8 & 40 & 4.76 & 22.5 & 32.5 & 38.3 & 48.1 & 51.2 \\
\hline 9 & 40 & 4.10 & 24.3 & 31.4 & 35.5 & 41.7 & 45.7 \\
\hline 10 & 40 & 3.65 & 24.8 & 33.8 & 40.4 & 43.5 & 44.8 \\
\hline 11 & 40 & 3.42 & 25.2 & 30.0 & 33.4 & 44.0 & 63.2 \\
\hline \multirow[t]{3}{*}{12} & 40 & 4.28 & 26.3 & 34.2 & 42.6 & 52.0 & 56.6 \\
\hline & $\overline{\mathbf{x}}$ & 4.15 & 23.5 & 31.3 & 37.1 & 45.2 & 51.0 \\
\hline & \pm sem & 0.2 & 1.2 & 1.2 & 1.6 & 1.7 & \\
\hline 13 & 50 & 4.58 & 22.7 & 26.5 & 33.2 & 44.7 & 44.7 \\
\hline 14 & 50 & 5.66 & 21.1 & 25.8 & 34.1 & 48.5 & 51.6 \\
\hline 15 & 50 & 4.76 & 26.5 & 32.4 & 37.0 & 46.6 & 46.6 \\
\hline 16 & 50 & 2.96 & 30.0 & 40.0 & 53.1 & & 57.0 \\
\hline 17 & 50 & 3.08 & 24.5 & 30.4 & 36.6 & 41.2 & 41.2 \\
\hline \multirow[t]{3}{*}{18} & 50 & 3.47 & 28.6 & 35.2 & 41.1 & & 41.1 \\
\hline & $\overline{\mathbf{x}}$ & 4.08 & 25.6 & 31.7 & 39.2 & 45.2 & 47.0 \\
\hline & tsem & 0.44 & 1.4 & 2.2 & 3.0 & 1.6 & 2.5 \\
\hline 19 & 60 & 3.82 & 23.0 & 32.2 & 35.9 & 40.1 & 40.1 \\
\hline 20 & 60 & 4.00 & 21.0 & 27.2 & 33.5 & & 40.7 \\
\hline 21 & 60 & 4.92 & 29.3 & 35.6 & 44.8 & & +4.8 \\
\hline 22 & 60 & 3.19 & 21.7 & 29.3 & 45.8 & & 45.8 \\
\hline \multirow[t]{2}{*}{23} & 60 & 3.62 & 25.8 & 39.1 & 49.4 & & 49.4 \\
\hline & $\begin{array}{c}\overline{\mathbf{x}} \\
\text { tsem }\end{array}$ & $\begin{array}{c}3.91 \\
03\end{array}$ & 24.2 & 32.7 & 41.9 & 40.1 & 44.2 \\
\hline
\end{tabular}


TABLE 5. Oxygen Consumption $\left(1 \cdot \mathrm{min}^{-1}\right)$

\begin{tabular}{|c|c|c|c|c|c|c|c|}
\hline \multirow[t]{2}{*}{ Participant } & \multirow[t]{2}{*}{ Group } & \multicolumn{6}{|c|}{ HR } \\
\hline & & Rest & 110 & 130 & 150 & 170 & $\operatorname{Max}$ \\
\hline 1 & 20 & 0.257 & 1.230 & 1.722 & 2.102 & 3.060 & 3.640 \\
\hline 2 & 20 & 0.329 & 1.702 & 1.945 & 2.400 & 2.901 & 3.965 \\
\hline 3 & 20 & 0.238 & 1.090 & 1.514 & 2.045 & 2.495 & 3.231 \\
\hline 4 & 20 & 0.159 & 1.206 & 1.746 & 2.708 & 3.165 & 4.068 \\
\hline 5 & 20 & 0.201 & 1.178 & 1.530 & 2.077 & 2.447 & 4.186 \\
\hline \multirow[t]{3}{*}{6} & 20 & 0.269 & 1.692 & 1.780 & 2.384 & 2.812 & 3.984 \\
\hline & $\overline{\mathbf{x}}$ & 0.242 & 1.350 & 1.706 & 2.286 & 2.813 & 3.846 \\
\hline & \pm sem & 0.024 & 0.112 & 0.066 & 0.106 & 0.119 & 0.144 \\
\hline 7 & 40 & 0.299 & 1.150 & 1.650 & 2.128 & 2.656 & 2.851 \\
\hline 8 & 40 & 0.288 & 1.363 & 1.962 & 2.314 & 2.906 & 3.092 \\
\hline 9 & 40 & 0.271 & 1.591 & 2.048 & 2.324 & 2.728 & 2.984 \\
\hline 10 & 40 & 0.231 & 1.564 & 2.133 & 2.546 & 2.744 & 2.831 \\
\hline 11 & 40 & 0.228 & 1.632 & 1.950 & 2.160 & 2.845 & 4.083 \\
\hline \multirow[t]{3}{*}{12} & 40 & 0.250 & 1.541 & 2.000 & 2.491 & 3.046 & 3.313 \\
\hline & $\bar{x}$ & 0.261 & 1.474 & 1.957 & 2.327 & 2.820 & 3.192 \\
\hline & 土sem & 0.012 & 0.075 & 0.067 & 0.069 & 0.058 & 0.192 \\
\hline 13 & 50 & 0.276 & 1.370 & 1.595 & 1.998 & 2.686 & 2.686 \\
\hline 14 & 50 & 0.316 & 1.182 & 1.449 & 1.911 & 2.719 & 2.877 \\
\hline 15 & 50 & 0.319 & 1.776 & 2.176 & 2.480 & 3.130 & 3.130 \\
\hline 16 & 50 & 0.172 & 1.745 & 2.338 & 3.106 & & 3.336 \\
\hline 17 & 50 & 0.209 & 1.666 & 2.056 & 2.472 & 2.785 & 2.785 \\
\hline \multirow[t]{3}{*}{18} & 50 & 0.197 & 1.626 & 2.003 & 2.344 & & 2.344 \\
\hline & $\overline{\mathbf{x}}$ & 0.248 & 1.561 & 1.936 & 2.385 & 2.830 & 2.860 \\
\hline & \pm sem & 0.026 & 0.096 & 0.140 & 0.174 & 0.102 & 0.142 \\
\hline 19 & 60 & 0.223 & 1.342 & 1.876 & 2.096 & 2.341 & 2.341 \\
\hline 20 & 60 & 0.216 & 1.140 & 1.475 & 1.815 & & 2.227 \\
\hline 21 & 60 & 0.252 & 1.496 & 1.818 & 2.288 & & 2.288 \\
\hline 22 & 60 & 0.215 & 1.466 & 1.978 & 3.090 & & 3.090 \\
\hline \multirow[t]{3}{*}{23} & 60 & 0.195 & 1.336 & 2.079 & 2.623 & & 2.623 \\
\hline & $\bar{x}$ & 0.220 & 1.356 & 1.845 & 2.382 & $2.34 \mathrm{l}$ & 2.514 \\
\hline & \pm sem & 0.009 & 0.063 & 0.103 & 0.220 & 0 & 0.159 \\
\hline
\end{tabular}


TABLE 6. Cardiac Output $\left(1 \cdot \mathrm{min}^{-1}\right)$

\begin{tabular}{|c|c|c|c|c|c|c|c|}
\hline \multirow[t]{2}{*}{ Participant } & \multirow[t]{2}{*}{ Group } & \multicolumn{6}{|c|}{ HR } \\
\hline & & Rest & 110 & 130 & 150 & 170 & $\operatorname{Max}$ \\
\hline 1 & 20 & 3.68 & 10.4 & 12.8 & 16.4 & 20.6 & 22.7 \\
\hline 2 & 20 & 3.83 & 11.2 & 14.1 & 16.5 & 18.4 & 23.2 \\
\hline 3 & 20 & 3.21 & 8.51 & 10.3 & 13.7 & 15.6 & 21.6 \\
\hline 4 & 20 & 1.95 & 8.10 & 11.3 & 17.1 & 18.9 & 23.3 \\
\hline 5 & 20 & 2.89 & 8.86 & 11.0 & 13.9 & 15.8 & 25.8 \\
\hline \multirow[t]{2}{*}{6} & 20 & 3.14 & 13.3 & 15.2 & 19.4 & 21.1 & 27.9 \\
\hline & $\begin{array}{c}\overline{\mathbf{x}} \\
\pm \text { sem }\end{array}$ & $\begin{array}{l}3.12 \\
0.27\end{array}$ & $\begin{array}{c}10.1 \\
0.8\end{array}$ & $\begin{array}{c}12.4 \\
0.8\end{array}$ & $\begin{array}{c}16.2 \\
0.9\end{array}$ & $\begin{array}{c}18.4 \\
0.9\end{array}$ & $\begin{array}{c}24.1 \\
0.9\end{array}$ \\
\hline 7 & 40 & 3.37 & 9.70 & 12.0 & 14.6 & 17.7 & 18.7 \\
\hline 8 & 40 & 2.73 & 9.53 & 14.2 & 15.9 & 18.1 & 19.3 \\
\hline 9 & 40 & 2.90 & 10.2 & 12.7 & 14.6 & 15.8 & 18.2 \\
\hline 10 & 40 & 2.40 & 10.5 & 12.9 & 15.3 & 16.9 & 17.5 \\
\hline 11 & 40 & 1.91 & 11.5 & 13.1 & 15.6 & 18.6 & 25.2 \\
\hline \multirow[t]{3}{*}{12} & 40 & 2.30 & 9.76 & 12.7 & 15.8 & 17.1 & 18.8 \\
\hline & $\overline{\mathbf{x}}$ & 2.60 & 10.2 & 12.9 & 15.3 & 17.4 & 19.6 \\
\hline & tsem & 0.2 & 0.3 & 0.3 & 0.2 & 0.4 & I.1 \\
\hline 13 & 50 & 3.45 & 9.98 & 11.7 & 12.3 & 16.2 & 16.2 \\
\hline 14 & 50 & 3.18 & 8.48 & 10.8 & 12.4 & 15.6 & 16.8 \\
\hline 15 & 50 & 3.22 & 10.9 & 13.3 & 15.3 & 18.8 & 18.8 \\
\hline 16 & 50 & 2.35 & 12.0 & 14.4 & 18.7 & & 21.1 \\
\hline 17 & 50 & 2.23 & 11.1 & 13.9 & 16.4 & 18.5 & 18.5 \\
\hline \multirow[t]{3}{*}{18} & 50 & 2.34 & 11.3 & 12.6 & 14.6 & & 14.6 \\
\hline & $\overline{\mathbf{x}}$ & 2.80 & 10.6 & 12.8 & 15.0 & 17.3 & 17.7 \\
\hline & tsem & 0.22 & 0.5 & 0.6 & 1.0 & 0.8 & 0.9 \\
\hline 19 & 60 & 2.22 & 8.72 & 11.9 & 13.6 & 15.2 & 15.2 \\
\hline 20 & 60 & $2.5 \mathrm{I}$ & 8.24 & 11.1 & 13.3 & & 14.8 \\
\hline 21 & 60 & 2.66 & 9.74 & 11.3 & 14.3 & & 14.3 \\
\hline 22 & 60 & 2.23 & 10.2 & 13.1 & 19.0 & & 19.0 \\
\hline \multirow[t]{2}{*}{23} & 60 & 1.72 & 9.25 & 13.8 & 16.3 & & 16.3 \\
\hline & $\begin{array}{c}\overline{\mathbf{x}} \\
\pm \text { sem }\end{array}$ & $\begin{array}{l}2.27 \\
0.16\end{array}$ & $\begin{array}{l}9.22 \\
0.34\end{array}$ & $\begin{array}{c}12.2 \\
0.5\end{array}$ & $\begin{array}{c}15.3 \\
1.1\end{array}$ & $\begin{array}{c}15.2 \\
0\end{array}$ & $\begin{array}{c}15.9 \\
0.8\end{array}$ \\
\hline
\end{tabular}


TABLE 7. Stroke Volume (ml)

\begin{tabular}{|c|c|c|c|c|c|c|c|}
\hline \multirow[t]{2}{*}{ Participant } & \multirow[t]{2}{*}{ Group } & \multicolumn{6}{|c|}{$\mathbf{H R}$} \\
\hline & & Rest & 110 & 130 & 150 & 170 & $\operatorname{Max}$ \\
\hline 1 & 20 & 52.9 & 94.8 & 98.2 & 109.3 & 121.6 & 123.5 \\
\hline 2 & 20 & 64.0 & 103.2 & 110.0 & 110.0 & 108.2 & 120.8 \\
\hline 3 & 20 & 44.6 & 76.3 & 80.8 & 90.1 & 91.9 & 114.3 \\
\hline 4 & 20 & 26.8 & 73.4 & 86.0 & 113.1 & 111.5 & 117.1 \\
\hline 5 & 20 & 35.3 & 79.2 & 84.2 & 92.4 & 92.1 & 131.2 \\
\hline \multirow[t]{2}{*}{6} & 20 & 44.7 & 119.9 & 115.5 & 128.7 & 123.5 & 145.5 \\
\hline & $\begin{array}{c}\overline{\mathbf{x}} \\
\pm \text { sem }\end{array}$ & $\begin{array}{c}44.7 \\
5.3\end{array}$ & $\begin{array}{c}91.1 \\
7.4\end{array}$ & $\begin{array}{c}95.8 \\
5.9\end{array}$ & $\begin{array}{c}107.3 \\
5.8\end{array}$ & $\begin{array}{c}108.1 \\
5.6\end{array}$ & $\begin{array}{c}125.4 \\
4.7\end{array}$ \\
\hline 7 & 40 & 51.5 & 87.8 & 93.6 & 97.9 & 104.0 & 106.0 \\
\hline 8 & 40 & 32.8 & 85.0 & 104.4 & 108.6 & 106.5 & 106.2 \\
\hline 9 & 40 & 46.1 & 92.9 & 98.0 & 95.5 & 93.4 & 102.7 \\
\hline 10 & 40 & 29.7 & 95.0 & 95.0 & 99.5 & 100.5 & 97.1 \\
\hline 11 & 40 & 27.5 & 103.8 & 101.1 & 104.2 & 108.6 & 136.3 \\
\hline \multirow[t]{3}{*}{12} & 40 & 31.7 & 88.3 & 97.9 & 105.4 & 103.2 & 108.0 \\
\hline & $\overline{\mathbf{x}}$ & 36.5 & 92.1 & 98.3 & 101.8 & 102.7 & 109.4 \\
\hline & & & & & & & \\
\hline 13 & 50 & 48.2 & 90.2 & 88.3 & 81.1 & 94.6 & 94.6 \\
\hline 14 & 50 & 41.3 & 77.1 & 82.5 & 81.2 & 91.7 & 96.5 \\
\hline 15 & 50 & 48.4 & 98.4 & 104.1 & 102.9 & 115.5 & 115.5 \\
\hline 16 & 50 & 33.7 & 108.3 & 110.5 & 124.6 & & 132.0 \\
\hline 17 & 50 & 36.7 & 100.6 & 107.8 & 109.3 & 108.1 & 108.1 \\
\hline \multirow[t]{2}{*}{18} & 50 & 38.5 & 102.9 & 97.2 & 95.2 & & 95.2 \\
\hline & $\begin{array}{c}\bar{x} \\
\pm \text { sem }\end{array}$ & $\begin{array}{c}41.1 \\
2.5\end{array}$ & $\begin{array}{c}96.2 \\
4.5\end{array}$ & $\begin{array}{c}98.4 \\
4.6\end{array}$ & $\begin{array}{c}99.0 \\
6.9\end{array}$ & $\begin{array}{c}102.5 \\
5.6\end{array}$ & $\begin{array}{c}107.0 \\
6.1\end{array}$ \\
\hline 19 & 60 & 36.7 & 79.7 & 90.9 & 90.5 & 90.5 & 90.5 \\
\hline 20 & 60 & 31.4 & 73.7 & 85.3 & 89.8 & & 93.9 \\
\hline 21 & 60 & 45.6 & 88.3 & 87.2 & 96.4 & & 96.4 \\
\hline 22 & 60 & 27.3 & 92.5 & 101.3 & 129.7 & & 129.7 \\
\hline \multirow[t]{2}{*}{23} & 60 & 19.0 & 82.0 & 105.1 & 109.6 & & 109.6 \\
\hline & $\begin{array}{c}\overline{\mathbf{x}} \\
\pm \text { sem }\end{array}$ & $\begin{array}{c}32.0 \\
4.5\end{array}$ & $\begin{array}{c}83.2 \\
3.3\end{array}$ & $\begin{array}{c}94.0 \\
3.9\end{array}$ & $\begin{array}{c}103.2 \\
7.5\end{array}$ & $\begin{array}{c}90.5 \\
0\end{array}$ & $\begin{array}{c}104.0 \\
7.2\end{array}$ \\
\hline
\end{tabular}


TABLE 8. Arterial Mixed - Venous Oxygen Difference $\left(\mathrm{ml} \mathrm{O}_{2} \cdot \mathrm{dl}^{\mathrm{ll}}\right.$ blood $\left.{ }^{-1}\right)$

\begin{tabular}{|c|c|c|c|c|c|c|c|}
\hline \multirow[t]{2}{*}{ Participant } & \multirow[t]{2}{*}{ Group } & \multicolumn{6}{|c|}{ HR } \\
\hline & & Rest & 110 & 130 & 150 & 170 & $\operatorname{Max}$ \\
\hline 1 & 20 & 69.84 & 118.3 & 134.5 & 128.2 & 148.5 & 160.6 \\
\hline 2 & 20 & 85.90 & 152.0 & 137.9 & 145.4 & 157.7 & 171.3 \\
\hline 3 & 20 & 74.14 & 128.1 & 147.0 & 149.3 & 159.9 & 149.7 \\
\hline 4 & 20 & 81.54 & 148.9 & 154.5 & 158.4 & 167.5 & 175.0 \\
\hline 5 & 20 & 69.55 & 133.0 & 139.1 & 149.4 & 154.9 & 162.0 \\
\hline \multirow[t]{2}{*}{6} & 20 & 85.67 & 127.2 & 117.1 & 122.9 & 133.3 & 142.0 \\
\hline & $\begin{array}{c}\overline{\mathbf{x}} \\
\pm \mathrm{sem}\end{array}$ & $\begin{array}{c}77.77 \\
3.09\end{array}$ & $\begin{array}{c}134.6 \\
5.4\end{array}$ & $\begin{array}{c}138.4 \\
5.2\end{array}$ & $\begin{array}{c}142.3 \\
5.6 \\
\end{array}$ & $\begin{array}{c}153.6 \\
4.8\end{array}$ & $\begin{array}{c}160.1 \\
5.1\end{array}$ \\
\hline 7 & 40 & 88.72 & 118.6 & 137.5 & 145.8 & 150.1 & 152.4 \\
\hline 8 & 40 & 105.2 & 143.1 & 140.1 & 145.9 & 160.6 & 160.0 \\
\hline 9 & 40 & 93.45 & 156.0 & 161.2 & 159.2 & 172.6 & 164.0 \\
\hline 10 & 40 & 96.25 & 149.0 & 165.3 & 138.5 & 162.4 & 161.8 \\
\hline 11 & 40 & 119.47 & 142.0 & 148.8 & 156.5 & 153.0 & 162.0 \\
\hline \multirow[t]{2}{*}{12} & 40 & 108.8 & 158.0 & 157.1 & 158.0 & 177.7 & 176.4 \\
\hline & $\begin{array}{c}\overline{\mathbf{x}} \\
\pm \text { sem } \\
\end{array}$ & $\begin{array}{c}102.0 \\
4.6\end{array}$ & $\begin{array}{c}144.4 \\
5.8 \\
\end{array}$ & $\begin{array}{c}151.7 \\
4.6\end{array}$ & $\begin{array}{c}150.7 \\
3.4\end{array}$ & $\begin{array}{c}162.7 \\
4.4 \\
\end{array}$ & $\begin{array}{c}162.8 \\
3.2 \\
\end{array}$ \\
\hline 13 & 50 & 80.0 & 137.3 & 136.3 & 162.4 & 165.8 & 165.8 \\
\hline 14 & 50 & 99.37 & 139.4 & 134.2 & 154.1 & 174.3 & 171.2 \\
\hline 15 & 50 & 99.07 & 162.9 & 151.1 & 162.1 & 166.5 & 166.5 \\
\hline 16 & 50 & 73.19 & 145.4 & 162.4 & 166.1 & & 158.1 \\
\hline 17 & 50 & 93.72 & 150.1 & 147.9 & 150.7 & 150.5 & 150.5 \\
\hline \multirow[t]{2}{*}{18} & 50 & 84.19 & 143.9 & 159.0 & 160.5 & & 160.5 \\
\hline & $\begin{array}{c}\overline{\mathbf{x}} \\
\pm \text { sem } \\
\end{array}$ & $\begin{array}{c}88.26 \\
4.40\end{array}$ & $\begin{array}{c}146.5 \\
3.8\end{array}$ & $\begin{array}{c}148.5 \\
4.7\end{array}$ & $\begin{array}{c}159.3 \\
2.4\end{array}$ & $\begin{array}{c}164.3 \\
5.0\end{array}$ & $\begin{array}{c}162.1 \\
3.0\end{array}$ \\
\hline 19 & 60 & 100.45 & 153.9 & 157.6 & 154.1 & 154.0 & 154.0 \\
\hline 20 & 60 & 86.06 & 138.3 & 132.9 & 136.5 & & 150.5 \\
\hline 21 & 60 & 94.74 & 153.6 & 160.9 & 160.0 & & 160.0 \\
\hline 22 & 60 & 97.05 & 144.3 & 151.8 & 162.7 & & 162.7 \\
\hline \multirow[t]{2}{*}{23} & 60 & 100.41 & 149.6 & 151.4 & 161.0 & & 161.0 \\
\hline & $\begin{array}{c}\overline{\mathbf{x}} \\
\pm \mathrm{sem}\end{array}$ & $\begin{array}{c}95.74 \\
2.6\end{array}$ & $\begin{array}{c}147.9 \\
3.0\end{array}$ & $\begin{array}{c}150.9 \\
4.8\end{array}$ & $\begin{array}{c}154.9 \\
4.8\end{array}$ & $\begin{array}{c}154.0 \\
0\end{array}$ & $\begin{array}{c}157.6 \\
2.3\end{array}$ \\
\hline
\end{tabular}


TABLE 9. Left Ventricular Ejection Time (msec)

\begin{tabular}{|c|c|c|c|c|c|c|c|}
\hline \multirow[t]{2}{*}{ Participant } & \multirow[t]{2}{*}{ Group } & \multicolumn{6}{|c|}{ HR } \\
\hline & & Rest & 110 & 130 & 150 & 170 & Max \\
\hline $\begin{array}{l}1 \\
2 \\
3 \\
4 \\
5 \\
6\end{array}$ & $\begin{array}{l}20 \\
20 \\
20 \\
20 \\
20 \\
20\end{array}$ & $\begin{array}{l}351 \\
356 \\
363 \\
362 \\
356 \\
365\end{array}$ & $\begin{array}{l}253 \\
260 \\
256 \\
242 \\
222 \\
261\end{array}$ & $\begin{array}{l}210 \\
231 \\
228 \\
220 \\
200 \\
234\end{array}$ & $\begin{array}{l}210 \\
221 \\
208 \\
192 \\
186 \\
210\end{array}$ & $\begin{array}{l}197 \\
202 \\
203 \\
186 \\
170 \\
204\end{array}$ & $\begin{array}{l}180 \\
179 \\
190 \\
166 \\
155 \\
180\end{array}$ \\
\hline & $\begin{array}{c}\overline{\mathbf{x}} \\
\pm \text { sem }\end{array}$ & $\begin{array}{c}359 \\
2\end{array}$ & $\begin{array}{c}249 \\
6\end{array}$ & $\begin{array}{c}220 \\
5\end{array}$ & $\begin{array}{c}204 \\
5\end{array}$ & $\begin{array}{c}194 \\
5\end{array}$ & $\begin{array}{c}175 \\
5\end{array}$ \\
\hline $\begin{array}{c}7 \\
8 \\
9 \\
10 \\
11 \\
12\end{array}$ & $\begin{array}{l}40 \\
40 \\
40 \\
40 \\
40 \\
40\end{array}$ & $\begin{array}{l}370 \\
367 \\
396 \\
362 \\
355 \\
365\end{array}$ & $\begin{array}{l}266 \\
254 \\
272 \\
266 \\
226 \\
262\end{array}$ & $\begin{array}{l}226 \\
243 \\
246 \\
238 \\
210 \\
224\end{array}$ & $\begin{array}{l}219 \\
228 \\
239 \\
219 \\
190 \\
185\end{array}$ & $\begin{array}{l}190 \\
208 \\
197 \\
205 \\
172 \\
194\end{array}$ & $\begin{array}{l}189 \\
190 \\
202 \\
176 \\
166 \\
187\end{array}$ \\
\hline & $\begin{array}{c}\overline{\mathrm{x}} \\
\pm \mathrm{sem}\end{array}$ & $\begin{array}{c}369 \\
6\end{array}$ & $\begin{array}{c}258 \\
7\end{array}$ & $\begin{array}{c}231 \\
6\end{array}$ & $\begin{array}{c}213 \\
9\end{array}$ & $\begin{array}{c}194 \\
5\end{array}$ & $\begin{array}{c}185 \\
5\end{array}$ \\
\hline $\begin{array}{l}13 \\
14 \\
15 \\
16 \\
17 \\
18\end{array}$ & $\begin{array}{l}50 \\
50 \\
50 \\
50 \\
50 \\
50\end{array}$ & $\begin{array}{l}374 \\
364 \\
363 \\
385 \\
358 \\
396\end{array}$ & $\begin{array}{l}242 \\
259 \\
261 \\
276 \\
263 \\
270\end{array}$ & $\begin{array}{l}220 \\
242 \\
252 \\
246 \\
238 \\
247\end{array}$ & $\begin{array}{l}198 \\
220 \\
230 \\
222 \\
213 \\
220\end{array}$ & $\begin{array}{r}178 \\
200 \\
220 \\
196\end{array}$ & $\begin{array}{l}178 \\
175 \\
220 \\
210 \\
196 \\
220\end{array}$ \\
\hline & $\begin{array}{c}\bar{x} \\
\text { tsem }\end{array}$ & $\begin{array}{c}373 \\
6 \\
\end{array}$ & $\begin{array}{c}262 \\
5\end{array}$ & $\begin{array}{c}241 \\
4\end{array}$ & $\begin{array}{c}217 \\
4\end{array}$ & $\begin{array}{c}198 \\
9 \\
\end{array}$ & $\begin{array}{c}200 \\
8 \\
\end{array}$ \\
\hline $\begin{array}{l}19 \\
20 \\
21 \\
22 \\
23\end{array}$ & $\begin{array}{l}60 \\
60 \\
60 \\
60 \\
60\end{array}$ & $\begin{array}{l}373 \\
360 \\
394 \\
403 \\
361\end{array}$ & $\begin{array}{l}258 \\
242 \\
257 \\
285 \\
236\end{array}$ & $\begin{array}{l}238 \\
227 \\
231 \\
260 \\
229\end{array}$ & $\begin{array}{l}226 \\
213 \\
204 \\
238 \\
205\end{array}$ & 211 & $\begin{array}{l}211 \\
190 \\
204 \\
238 \\
205\end{array}$ \\
\hline & $\begin{array}{c}\overline{\mathbf{x}} \\
\text { 土sem }\end{array}$ & $\begin{array}{c}378 \\
9\end{array}$ & $\begin{array}{c}256 \\
8\end{array}$ & $\begin{array}{c}237 \\
6\end{array}$ & $\begin{array}{c}217 \\
6\end{array}$ & $\begin{array}{c}211 \\
0\end{array}$ & $\begin{array}{c}210 \\
8\end{array}$ \\
\hline
\end{tabular}


TABLE 10. Pre-Ejection Period (msec)

\begin{tabular}{|c|c|c|c|c|c|c|c|}
\hline \multirow[t]{2}{*}{ Participant } & \multirow[t]{2}{*}{ Group } & \multicolumn{6}{|c|}{ HR } \\
\hline & & Rest & 110 & 130 & 150 & 170 & $\operatorname{Max}$ \\
\hline 1 & 20 & 212 & 93 & 65 & 56 & 35 & 47 \\
\hline 2 & 20 & 213 & 55 & 26 & 8 & 24 & 25 \\
\hline 3 & 20 & 199 & 87 & 42 & 34 & 11 & 22 \\
\hline 4 & 20 & 258 & 32 & 39 & 64 & 43 & 23 \\
\hline 5 & 20 & 223 & 72 & 65 & 79 & 69 & 47 \\
\hline & 20 & 165 & 59 & 66 & 47 & 30 & 24 \\
\hline & $\begin{array}{c}\overline{\mathbf{x}} \\
\pm \mathrm{sem}\end{array}$ & $\begin{array}{c}212 \\
12\end{array}$ & $\begin{array}{c}66 \\
9\end{array}$ & $\begin{array}{c}50 \\
7\end{array}$ & $\begin{array}{l}48 \\
10\end{array}$ & $\begin{array}{c}35 \\
8\end{array}$ & $\begin{array}{c}31 \\
5\end{array}$ \\
\hline 7 & 40 & 240 & 54 & 56 & 10 & 27 & 19 \\
\hline 8 & 40 & 178 & 36 & 27 & 10 & 12 & 13 \\
\hline 9 & 40 & 253 & 91 & 28 & 36 & 37 & 21 \\
\hline 10 & 40 & 227 & 92 & 35 & 23 & 17 & 40 \\
\hline 11 & 40 & 238 & 74 & 52 & 47 & 13 & 12 \\
\hline \multirow[t]{3}{*}{12} & 40 & 211 & 53 & 47 & 45 & 18 & 15 \\
\hline & $\overline{\mathbf{x}}$ & 224 & 67 & 41 & 28 & 21 & 20 \\
\hline & $\pm \mathrm{sem}$ & 11 & 9 & 5 & 7 & 4 & 4 \\
\hline 13 & 50 & 205 & 74 & 37 & 35 & 48 & 48 \\
\hline 14 & 50 & 169 & 93 & 50 & 26 & 24 & 43 \\
\hline 15 & 50 & 253 & 76 & 32 & 37 & 13 & 13 \\
\hline 16 & 50 & 149 & 6 & 33 & 41 & & 43 \\
\hline 17 & 50 & 200 & 39 & 38 & 23 & 16 & 16 \\
\hline \multirow[t]{3}{*}{18} & 50 & 230 & 22 & 27 & 14 & & 14 \\
\hline & $\overline{\mathbf{x}}$ & 201 & 52 & 36 & 29 & 25 & 30 \\
\hline & $\pm \mathrm{sem}$ & 16 & 14 & 3 & 4 & 8 & 7 \\
\hline 19 & 60 & 243 & 82 & 38 & 32 & 28 & 28 \\
\hline 20 & 60 & 224 & 92 & 44 & 25 & & 35 \\
\hline 21 & 60 & 253 & 77 & 51 & 49 & & 49 \\
\hline 22 & 60 & 176 & 32 & 25 & 10 & & 10 \\
\hline \multirow[t]{3}{*}{23} & 60 & 196 & 61 & 42 & 45 & & 45 \\
\hline & $\overline{\mathbf{x}}$ & 218 & 69 & 40 & 32 & 28 & 33 \\
\hline & $\pm \mathrm{sem}$ & 14 & 10 & 4 & 7 & 0 & 7 \\
\hline
\end{tabular}


TABLE 11. Diastolic Filling Time (msec)

\begin{tabular}{|c|c|c|c|c|c|c|c|}
\hline \multirow[t]{2}{*}{ Participant } & \multirow[t]{2}{*}{ Group } & \multicolumn{6}{|c|}{ HR } \\
\hline & & Rest & 110 & 130 & 150 & 170 & $\operatorname{Max}$ \\
\hline 1 & 20 & 505 & 200 & 160 & 139 & 122 & 110 \\
\hline 2 & 20 & 464 & 223 & 201 & 168 & 128 & 114 \\
\hline 3 & 20 & 524 & 200 & 182 & 152 & 137 & 104 \\
\hline 4 & 20 & 531 & 230 & 189 & 139 & 121 & 115 \\
\hline 5 & 20 & 375 & 254 & 188 & 129 & 109 & 108 \\
\hline \multirow[t]{2}{*}{6} & 20 & 618 & 242 & 180 & 154 & 139 & 114 \\
\hline & $\begin{array}{c}\overline{\mathbf{x}} \\
\pm \mathrm{sem}\end{array}$ & $\begin{array}{c}503 \\
33\end{array}$ & $\begin{array}{c}225 \\
9\end{array}$ & $\begin{array}{c}183 \\
6\end{array}$ & $\begin{array}{c}147 \\
6\end{array}$ & $\begin{array}{c}126 \\
4\end{array}$ & $\begin{array}{c}111 \\
2\end{array}$ \\
\hline 7 & 40 & 548 & 226 & 156 & 143 & 121 & 115 \\
\hline 8 & 40 & 427 & 268 & 210 & 189 & 145 & 135 \\
\hline 9 & 40 & 471 & 192 & 184 & 139 & 122 & 114 \\
\hline 10 & 40 & 318 & 200 & 180 & 164 & 129 & 118 \\
\hline 11 & 40 & 473 & 238 & 195 & 169 & 158 & 136 \\
\hline \multirow[t]{2}{*}{12} & 40 & 375 & 250 & 193 & 178 & 150 & 128 \\
\hline & $\begin{array}{c}\bar{x} \\
\pm \text { sem }\end{array}$ & $\begin{array}{c}435 \\
33\end{array}$ & $\begin{array}{c}229 \\
12\end{array}$ & $\begin{array}{c}186 \\
7\end{array}$ & $\begin{array}{c}164 \\
8\end{array}$ & $\begin{array}{c}138 \\
6\end{array}$ & $\begin{array}{c}124 \\
4\end{array}$ \\
\hline 13 & 50 & 492 & 227 & 214 & 170 & 128 & 128 \\
\hline 14 & 50 & 523 & 197 & 170 & 151 & 127 & 122 \\
\hline 15 & 50 & 492 & 226 & 199 & 131 & 123 & 123 \\
\hline 16 & 50 & 495 & 268 & 208 & 148 & & 134 \\
\hline 17 & 50 & 536 & 246 & 181 & 151 & 134 & 134 \\
\hline \multirow[t]{2}{*}{18} & 50 & 442 & 251 & 186 & 175 & & 175 \\
\hline & $\begin{array}{c}\overline{\mathbf{x}} \\
\pm \mathrm{sem}\end{array}$ & $\begin{array}{c}497 \\
13\end{array}$ & $\begin{array}{c}236 \\
10\end{array}$ & $\begin{array}{c}193 \\
7\end{array}$ & $\begin{array}{c}154 \\
6\end{array}$ & $\begin{array}{c}128 \\
2\end{array}$ & $\begin{array}{c}136 \\
8\end{array}$ \\
\hline 19 & 60 & 482 & 212 & 178 & 134 & 117 & 117 \\
\hline 20 & 60 & 353 & 235 & 187 & 162 & & 176 \\
\hline 21 & 60 & 603 & 207 & 178 & 141 & & $1+1$ \\
\hline 22 & 60 & 454 & 265 & 208 & 176 & & 176 \\
\hline \multirow[t]{3}{*}{23} & 60 & 550 & 260 & 202 & 160 & & 160 \\
\hline & $\overline{\mathbf{x}}$ & 488 & 236 & 191 & 155 & 117 & 154 \\
\hline & \pm sem & 43 & 12 & 6 & 8 & 0 & 11 \\
\hline
\end{tabular}


TABLE 12. Diastolic Filling Rate (ml- $\left.\mathrm{sec}^{-1}\right)$

\begin{tabular}{|c|c|c|c|c|c|c|c|}
\hline \multirow[t]{2}{*}{ Participant } & \multirow[t]{2}{*}{ Group } & \multicolumn{6}{|c|}{ HR } \\
\hline & & Rest & 110 & 130 & 150 & 170 & $\operatorname{Max}$ \\
\hline 1 & 20 & 105 & 474 & 614 & 786 & 997 & 1123 \\
\hline 2 & 20 & 138 & 463 & 547 & 655 & 845 & 1060 \\
\hline 3 & 20 & 85.0 & 382 & 444 & 593 & 671 & 1099 \\
\hline 4 & 20 & 50.5 & 319 & 455 & 814 & 921 & 1018 \\
\hline 5 & 20 & 94.0 & 312 & 448 & 716 & 845 & 1215 \\
\hline \multirow[t]{2}{*}{6} & 20 & 72.0 & 495 & 642 & 836 & 888 & 1276 \\
\hline & $\begin{array}{c}\overline{\mathbf{x}} \\
\text { tsem }\end{array}$ & $\begin{array}{l}90.4 \\
12.3\end{array}$ & $\begin{array}{c}358 \\
43\end{array}$ & $\begin{array}{c}525 \\
36\end{array}$ & $\begin{array}{c}733 \\
39\end{array}$ & $\begin{array}{c}861 \\
44\end{array}$ & $\begin{array}{c}1132 \\
40\end{array}$ \\
\hline 7 & 40 & 94.0 & 388 & 600 & 685 & 860 & 922 \\
\hline 8 & 40 & 76.7 & 318 & 497 & 575 & 734 & 786 \\
\hline 9 & 40 & 98.0 & 484 & 533 & 687 & 766 & 901 \\
\hline 10 & 40 & 93.0 & 475 & 528 & 607 & 779 & 823 \\
\hline 11 & 40 & 58.0 & 436 & 519 & 616 & 687 & 1002 \\
\hline \multirow[t]{3}{*}{12} & 40 & 84.5 & 353 & 507 & 592 & 688 & 843 \\
\hline & $\overline{\mathbf{x}}$ & 84.0 & 409 & 531 & 627 & 752 & 880 \\
\hline & \pm sem & 6 & 27 & 15 & 20 & 27 & 32 \\
\hline 13 & 50 & 98.0 & 397 & 413 & 477 & 739 & 739 \\
\hline 14 & 50 & 79.0 & 391 & 485 & 538 & 722 & 791 \\
\hline 15 & 50 & 98.4 & 435 & 523 & 785 & 939 & 939 \\
\hline 16 & 50 & 68.1 & 404 & 531 & 842 & & 985 \\
\hline 17 & 50 & 68.5 & 409 & 596 & 724 & 807 & 807 \\
\hline \multirow[t]{3}{*}{18} & 50 & 83.0 & 410 & 522 & 544 & & 544 \\
\hline & $\overline{\mathbf{x}}$ & 82.5 & 408 & 512 & 652 & 802 & 801 \\
\hline & \pm sem & 5.5 & 6 & 25 & 62 & 49 & 64 \\
\hline 19 & 60 & 76.0 & 376 & 511 & 675 & 774 & 774 \\
\hline 20 & 60 & 89.0 & 314 & 456 & 554 & & 534 \\
\hline 21 & 60 & 75.6 & 426 & 490 & 684 & & 684 \\
\hline 22 & 60 & 60.1 & 349 & 487 & 737 & & 737 \\
\hline \multirow[t]{2}{*}{23} & 60 & 34.0 & 315 & 520 & 685 & & 685 \\
\hline & $\begin{array}{c}\bar{x} \\
\pm \text { sem }\end{array}$ & $\begin{array}{c}66.9 \\
9.4\end{array}$ & $\begin{array}{c}356 \\
21\end{array}$ & $\begin{array}{c}493 \\
11\end{array}$ & $\begin{array}{c}667 \\
30\end{array}$ & $\begin{array}{c}774 \\
0\end{array}$ & $\begin{array}{c}683 \\
41\end{array}$ \\
\hline
\end{tabular}


TABLE 13. Left Ventricular Ejection Rate $\left(\mathrm{ml} \cdot \mathrm{sec}^{-1}\right)$

\begin{tabular}{|c|c|c|c|c|c|c|c|}
\hline \multirow[t]{2}{*}{ Participant } & \multirow[t]{2}{*}{ Group } & \multicolumn{6}{|c|}{ HR } \\
\hline & & Rest & 110 & 130 & 150 & 170 & $\operatorname{Max}$ \\
\hline 1 & 20 & 151 & 375 & 468 & 520 & 617 & 686 \\
\hline 2 & 20 & 180 & 397 & 476 & 498 & 536 & 675 \\
\hline 3 & 20 & 123 & 298 & 354 & 433 & 454 & 602 \\
\hline 4 & 20 & 74 & 303 & 391 & 589 & 599 & 705 \\
\hline 5 & 20 & 99 & 357 & 421 & 497 & 542 & 846 \\
\hline \multirow[t]{2}{*}{6} & 20 & 122 & 459 & 494 & 613 & 605 & 808 \\
\hline & $\begin{array}{c}\overline{\mathbf{x}} \\
\pm \mathrm{sem}\end{array}$ & $\begin{array}{c}124.8 \\
15.3\end{array}$ & $\begin{array}{c}365 \\
25\end{array}$ & $\begin{array}{c}434 \\
22\end{array}$ & $\begin{array}{l}525 \\
27\end{array}$ & $\begin{array}{l}559 \\
25\end{array}$ & $\begin{array}{c}720 \\
37\end{array}$ \\
\hline 7 & 40 & 139 & 330 & 414 & 447 & 547 & 561 \\
\hline 8 & 40 & 89 & 335 & 430 & 476 & 502 & 523 \\
\hline 9 & 40 & 116 & 342 & 398 & 400 & 474 & 508 \\
\hline 10 & 40 & 82 & 357 & 399 & 454 & 490 & 552 \\
\hline 11 & 40 & 77 & 459 & 481 & 548 & 631 & 821 \\
\hline \multirow[t]{3}{*}{12} & 40 & 87 & 337 & 437 & 570 & 532 & 577 \\
\hline & $\bar{x}$ & 98 & 360 & 426 & 482 & 529 & 590 \\
\hline & \pm sem & 10 & 20 & 13 & 26 & 23 & 47 \\
\hline 13 & 50 & 129 & 373 & 401 & 410 & 531 & 532 \\
\hline 14 & 50 & 113 & 298 & 341 & 369 & 458 & 551 \\
\hline 15 & 50 & 133 & 377 & 413 & 447 & 525 & 525 \\
\hline 16 & 50 & 88 & 392 & 449 & 561 & & 628 \\
\hline 17 & 50 & 102 & 382 & 453 & 513 & 552 & 552 \\
\hline \multirow[t]{2}{*}{18} & 50 & 97 & 381 & 394 & 433 & & 433 \\
\hline & $\begin{array}{c}\overline{\mathbf{x}} \\
\pm \mathrm{sem}\end{array}$ & $\begin{array}{c}110 \\
7\end{array}$ & $\begin{array}{c}367 \\
14\end{array}$ & $\begin{array}{c}392 \\
26\end{array}$ & $\begin{array}{c}456 \\
29\end{array}$ & $\begin{array}{c}516 \\
20\end{array}$ & $\begin{array}{r}537 \\
26\end{array}$ \\
\hline 19 & 60 & 98 & 309 & 382 & 400 & 429 & 429 \\
\hline 20 & 60 & 87 & 304 & 376 & 422 & & 494 \\
\hline 21 & 60 & 116 & 344 & 377 & 472 & & 472 \\
\hline 22 & 60 & 68 & 324 & 390 & 545 & & 545 \\
\hline \multirow[t]{3}{*}{23} & 60 & 53 & 347 & 459 & 535 & & 535 \\
\hline & $\overline{\mathbf{x}}$ & 84 & 326 & 397 & 475 & 429 & 495 \\
\hline & $\pm \mathrm{sem}$ & 11 & 9 & 16 & 29 & 0 & 21 \\
\hline
\end{tabular}


TABLE 14. Systolic Blood Pressure (mmHg)

\begin{tabular}{|c|c|c|c|c|c|c|c|}
\hline \multirow[t]{2}{*}{ Participant } & \multirow[t]{2}{*}{ Group } & \multicolumn{6}{|c|}{ HR } \\
\hline & & Rest & 110 & 130 & 150 & 170 & $\operatorname{Max}$ \\
\hline $\begin{array}{l}1 \\
2 \\
3 \\
4 \\
5 \\
6\end{array}$ & $\begin{array}{l}20 \\
20 \\
20 \\
20 \\
20 \\
20\end{array}$ & $\begin{array}{l}106 \\
102 \\
122 \\
109 \\
108 \\
121\end{array}$ & $\begin{array}{l}129 \\
122 \\
140 \\
129 \\
140 \\
134\end{array}$ & $\begin{array}{l}136 \\
158 \\
147 \\
135 \\
155 \\
152\end{array}$ & $\begin{array}{l}153 \\
174 \\
152 \\
151 \\
160 \\
156\end{array}$ & $\begin{array}{l}171 \\
196 \\
170 \\
166 \\
168 \\
177\end{array}$ & $\begin{array}{l}180 \\
207 \\
175 \\
175 \\
170 \\
186\end{array}$ \\
\hline & $\begin{array}{c}\overline{\mathbf{x}} \\
\pm \text { sem } \\
\end{array}$ & $\begin{array}{c}111 \\
3 \\
\end{array}$ & $\begin{array}{c}132 \\
3 \\
\end{array}$ & $\begin{array}{c}147 \\
4 \\
\end{array}$ & $\begin{array}{c}158 \\
4 \\
\end{array}$ & $\begin{array}{c}175 \\
4 \\
\end{array}$ & $\begin{array}{c}182 \\
5 \\
\end{array}$ \\
\hline $\begin{array}{c}7 \\
8 \\
9 \\
10 \\
11 \\
12\end{array}$ & $\begin{array}{l}40 \\
40 \\
40 \\
40 \\
40 \\
40\end{array}$ & $\begin{array}{c}120 \\
92 \\
112 \\
102 \\
130 \\
100\end{array}$ & $\begin{array}{l}141 \\
110 \\
135 \\
127 \\
156 \\
110\end{array}$ & $\begin{array}{l}170 \\
112 \\
154 \\
144 \\
168 \\
122\end{array}$ & $\begin{array}{l}174 \\
119 \\
174 \\
154 \\
167 \\
140\end{array}$ & $\begin{array}{l}178 \\
134 \\
189 \\
174 \\
176 \\
162\end{array}$ & $\begin{array}{l}180 \\
140 \\
198 \\
185 \\
185 \\
173\end{array}$ \\
\hline & $\begin{array}{c}\overline{\mathbf{x}} \\
\pm \text { sem }\end{array}$ & $\begin{array}{c}109 \\
6\end{array}$ & $\begin{array}{c}130 \\
7\end{array}$ & $\begin{array}{c}145 \\
10\end{array}$ & $\begin{array}{c}155 \\
9\end{array}$ & $\begin{array}{c}169 \\
8\end{array}$ & $\begin{array}{c}177 \\
8\end{array}$ \\
\hline $\begin{array}{l}13 \\
14 \\
15 \\
16 \\
17 \\
18\end{array}$ & $\begin{array}{l}50 \\
50 \\
50 \\
50 \\
50 \\
50\end{array}$ & $\begin{array}{l}114 \\
115 \\
108 \\
129 \\
105 \\
115\end{array}$ & $\begin{array}{l}128 \\
124 \\
125 \\
132 \\
140 \\
166\end{array}$ & $\begin{array}{l}143 \\
138 \\
149 \\
136 \\
178 \\
180\end{array}$ & $\begin{array}{l}158 \\
166 \\
165 \\
156 \\
179 \\
182\end{array}$ & $\begin{array}{c}166 \\
17 \\
179 \\
188\end{array}$ & $\begin{array}{l}166 \\
185 \\
179 \\
168 \\
188 \\
182\end{array}$ \\
\hline & $\begin{array}{c}\overline{\mathbf{x}} \\
\pm \text { sem } \\
\end{array}$ & $\begin{array}{c}114 \\
3 \\
\end{array}$ & $\begin{array}{c}136 \\
6 \\
\end{array}$ & $\begin{array}{c}154 \\
8 \\
\end{array}$ & $\begin{array}{c}168 \\
4 \\
\end{array}$ & $\begin{array}{c}177 \\
5 \\
\end{array}$ & $\begin{array}{c}178 \\
4 \\
\end{array}$ \\
\hline $\begin{array}{l}19 \\
20 \\
21 \\
22 \\
23\end{array}$ & $\begin{array}{l}60 \\
60 \\
60 \\
60 \\
60\end{array}$ & $\begin{array}{l}114 \\
116 \\
116 \\
112 \\
130\end{array}$ & $\begin{array}{l}130 \\
146 \\
157 \\
159 \\
164\end{array}$ & $\begin{array}{l}152 \\
167 \\
190 \\
181 \\
180\end{array}$ & $\begin{array}{l}170 \\
182 \\
211 \\
194 \\
201\end{array}$ & 172 & $\begin{array}{l}172 \\
181 \\
211 \\
194 \\
201\end{array}$ \\
\hline & $\begin{array}{c}\overline{\mathbf{x}} \\
\pm \text { sem }\end{array}$ & $\begin{array}{c}118 \\
3\end{array}$ & $\begin{array}{c}151 \\
6\end{array}$ & $\begin{array}{c}174 \\
7\end{array}$ & $\begin{array}{c}192 \\
7\end{array}$ & $\begin{array}{c}172 \\
0\end{array}$ & $\begin{array}{c}192 \\
7\end{array}$ \\
\hline
\end{tabular}


TABLE 15. Diastolic Blood Pressure (mmHg)

\begin{tabular}{|c|c|c|c|c|c|c|c|}
\hline \multirow[t]{2}{*}{ Participant } & \multirow[t]{2}{*}{ Group } & \multicolumn{6}{|c|}{ HR } \\
\hline & & Rest & 110 & 130 & 150 & 170 & $\operatorname{Max}$ \\
\hline 1 & 20 & 71 & 74 & 71 & 63 & 59 & 56 \\
\hline 2 & 20 & 69 & 65 & 60 & 69 & 67 & 71 \\
\hline 3 & 20 & 78 & 74 & 73 & 75 & 73 & 71 \\
\hline 4 & 20 & 61 & 60 & 59 & 63 & 60 & 63 \\
\hline 5 & 20 & 71 & 69 & 73 & 68 & 69 & 69 \\
\hline \multirow[t]{2}{*}{6} & 20 & 78 & 70 & 69 & 65 & 64 & 64 \\
\hline & $\begin{array}{c}\bar{x} \\
\pm \operatorname{sem}\end{array}$ & $\begin{array}{c}71 \\
2\end{array}$ & $\begin{array}{c}69 \\
2\end{array}$ & $\begin{array}{c}68 \\
3\end{array}$ & $\begin{array}{c}67 \\
2\end{array}$ & $\begin{array}{c}65 \\
2\end{array}$ & $\begin{array}{c}66 \\
2\end{array}$ \\
\hline 7 & 40 & 75 & 69 & 68 & 68 & 66 & 68 \\
\hline 8 & 40 & 70 & 66 & 66 & 66 & 64 & 64 \\
\hline 9 & 40 & 80 & 70 & 71 & 74 & 74 & 74 \\
\hline 10 & 40 & 70 & 66 & 64 & 67 & 62 & 63 \\
\hline 11 & 40 & 86 & 87 & 90 & 85 & 82 & 75 \\
\hline \multirow[t]{3}{*}{12} & 40 & 70 & 68 & 64 & 66 & 64 & 64 \\
\hline & $\overline{\mathbf{x}}$ & 75 & 71 & 70 & 71 & 69 & 68 \\
\hline & \pm sem & 3 & 3 & 4 & 3 & 3 & 2 \\
\hline 13 & 50 & 73 & 70 & 64 & 66 & 73 & 73 \\
\hline 14 & 50 & 76 & 78 & 74 & 74 & 70 & 78 \\
\hline 15 & 50 & 76 & 74 & 74 & 75 & 77 & 77 \\
\hline 16 & 50 & 77 & 76 & 74 & 76 & & 75 \\
\hline 17 & 50 & 76 & 73 & 66 & 69 & 64 & 64 \\
\hline \multirow[t]{3}{*}{18} & 50 & 76 & 75 & 76 & 68 & & 68 \\
\hline & $\overline{\mathbf{x}}$ & 76 & 74 & 71 & 71 & 71 & 72 \\
\hline & $\pm \mathrm{sem}$ & 1 & 1 & 2 & 2 & 3 & 2 \\
\hline 19 & 60 & 71 & 74 & 76 & 79 & 79 & 79 \\
\hline 20 & 60 & 81 & 73 & 68 & 71 & & 70 \\
\hline 21 & 60 & 72 & 76 & 79 & 78 & & 78 \\
\hline 22 & 60 & 73 & 70 & 81 & 79 & & 79 \\
\hline \multirow[t]{3}{*}{23} & 60 & 95 & 95 & 105 & 102 & & 102 \\
\hline & $\overline{\mathbf{x}}$ & 78 & 78 & 82 & 82 & 79 & 82 \\
\hline & $\pm \mathrm{sem}$ & 4 & 4 & 6 & 5 & 0 & 5 \\
\hline
\end{tabular}


TABLE 16. Mean Arterial Pressure ( $\mathrm{mmHg}$ )

\begin{tabular}{|c|c|c|c|c|c|c|c|}
\hline \multirow[t]{2}{*}{ Participant } & \multirow[t]{2}{*}{ Group } & \multicolumn{6}{|c|}{ HR } \\
\hline & & Rest & 110 & 130 & 150 & 170 & $\operatorname{Max}$ \\
\hline 1 & 20 & 82.7 & 92.3 & 92.7 & 93.0 & 96.3 & 97.5 \\
\hline 2 & 20 & 80.0 & 84.0 & 93.0 & 104.0 & 110.0 & 116.0 \\
\hline 3 & 20 & 92.7 & 96.0 & 97.7 & 101.0 & 105.0 & 106.0 \\
\hline 4 & 20 & 77.0 & 83.0 & 84.3 & 92.3 & 95.3 & 100.3 \\
\hline 5 & 20 & 83.3 & 92.7 & 100.3 & 98.7 & 102.0 & 102.7 \\
\hline \multirow[t]{2}{*}{6} & 20 & 92.3 & 91.3 & 96.7 & 95.3 & 101.7 & 104.7 \\
\hline & $\begin{array}{c}\overline{\mathbf{x}} \\
\pm \mathrm{sem}\end{array}$ & $\begin{array}{c}84.7 \\
2.6\end{array}$ & $\begin{array}{c}89.9 \\
2.1\end{array}$ & $\begin{array}{c}94.1 \\
2.3\end{array}$ & $\begin{array}{c}97.4 \\
1.9\end{array}$ & $\begin{array}{c}101.7 \\
2.2\end{array}$ & $\begin{array}{c}104.5 \\
2.6\end{array}$ \\
\hline 7 & 40 & 90.0 & 95.0 & 102.0 & 103.0 & 103.0 & 105.0 \\
\hline 8 & 40 & 77.3 & 80.7 & 81.3 & 83.7 & 87.3 & 89.3 \\
\hline 9 & 40 & 91.0 & 92.0 & 99.0 & 107.0 & 112.0 & 115.0 \\
\hline 10 & 40 & 81.0 & 86.0 & 91.0 & 96.0 & 99.0 & 104.0 \\
\hline 11 & 40 & 100.7 & 110.0 & 116.0 & 112.3 & 113.3 & 111.7 \\
\hline \multirow[t]{3}{*}{12} & 40 & 80.0 & 82.0 & 83.3 & 90.7 & 96.7 & 100.3 \\
\hline & $\overline{\mathbf{x}}$ & 86.7 & 91.0 & 95.4 & 98.8 & 101.9 & 104.2 \\
\hline & \pm sem & 3.6 & 4.4 & 5.3 & 4.4 & 4.0 & 3.7 \\
\hline 13 & 50 & 86.0 & 90.0 & 91.0 & 97.0 & 104.0 & 104.0 \\
\hline 14 & 50 & 89.0 & 93.0 & 95.0 & 103.0 & 106.0 & 112.0 \\
\hline 15 & 50 & 86.7 & 91.0 & 99.0 & 105.0 & 111.0 & 111.0 \\
\hline 16 & 50 & 94.3 & 94.7 & 94.7 & 103.0 & & 106.0 \\
\hline 17 & 50 & 86.0 & 95.0 & 103.0 & 106.0 & 105.0 & 105.0 \\
\hline \multirow[t]{3}{*}{18} & 50 & 89.0 & 105.0 & 111.0 & 106.0 & & 106.0 \\
\hline & $\overline{\mathbf{x}}$ & 88.5 & 94.8 & 99.0 & 103.0 & 106.0 & 107.0 \\
\hline & \pm sem & 1.3 & 2.2 & 2.9 & 1.0 & 2.0 & 1.0 \\
\hline 19 & 60 & 85.3 & 92.7 & 101.3 & 101.3 & 109.3 & 109.3 \\
\hline 20 & 60 & 92.7 & 97.3 & 101.0 & 108.0 & & 107.0 \\
\hline 21 & 60) & 86.7 & 103.0) & 116.0 & 122.0 & & 122.0 \\
\hline 22 & 60 & 86.0 & 100.0 & 114.0 & 117.0 & & 117.0 \\
\hline \multirow[t]{2}{*}{23} & 60 & 107.0 & 118.0 & 130.0 & 135.0 & & 135.0 \\
\hline & $\begin{array}{c}\bar{x} \\
\pm \text { sem }\end{array}$ & $\begin{array}{c}91.5 \\
4.1\end{array}$ & $\begin{array}{c}102.2 \\
4.3\end{array}$ & $\begin{array}{c}112.5 \\
5.4\end{array}$ & $\begin{array}{c}116.7 \\
5.8\end{array}$ & $\begin{array}{c}109.3 \\
0\end{array}$ & $\begin{array}{c}118.1 \\
5.0\end{array}$ \\
\hline
\end{tabular}


TABLE 17. Total Peripheral Resistance $\left(\mathrm{mmHg} \cdot \mathrm{l}^{-1} \cdot \mathrm{min}^{-1}\right)$

\begin{tabular}{|c|c|c|c|c|c|c|c|}
\hline \multirow[t]{2}{*}{ Participant } & \multirow[t]{2}{*}{ Group } & \multicolumn{6}{|c|}{ HR } \\
\hline & & Rest & 110 & 130 & 150 & 170 & $\operatorname{Max}$ \\
\hline 1 & 20 & 22.5 & 8.88 & 7.24 & 5.67 & 4.67 & 4.30 \\
\hline 2 & 20 & 20.9 & 7.50 & 6.60 & 6.30 & 5.98 & 5.00 \\
\hline 3 & 20 & 28.9 & 11.3 & 9.48 & 7.37 & 6.73 & 4.91 \\
\hline 4 & 20 & 39.5 & 10.2 & 7.46 & 5.40 & 5.04 & 4.30 \\
\hline 5 & 20 & 28.8 & 10.5 & 9.12 & 7.10 & 6.46 & 3.98 \\
\hline \multirow[t]{3}{*}{6} & 20 & 29.4 & 6.86 & 6.36 & 4.91 & 4.82 & 3.75 \\
\hline & $\overline{\mathbf{x}}$ & 28.3 & 9.21 & 7.71 & 6.12 & 5.62 & 4.37 \\
\hline & \pm sem & 2.7 & 0.72 & 0.53 & 0.40 & 0.36 & 0.20 \\
\hline 7 & 40 & 26.7 & 9.79 & 8.50 & 7.09 & 5.82 & 5.60 \\
\hline 8 & 40 & 28.3 & 8.47 & 5.74 & 5.28 & 4.82 & 4.62 \\
\hline 9 & 40 & 31.4 & 9.02 & 7.80 & 7.33 & 7.09 & 6.32 \\
\hline 10 & 40 & 33.8 & 8.19 & 7.05 & 6.27 & 5.86 & 5.94 \\
\hline 11 & 40 & 52.7 & 9.57 & 8.85 & 7.20 & 6.09 & 4.43 \\
\hline \multirow[t]{3}{*}{12} & 40 & 34.8 & 8.40 & 6.55 & 5.75 & 5.63 & 5.34 \\
\hline & $\overline{\mathbf{x}}$ & 34.6 & 8.91 & 7.42 & 6.48 & 5.88 & 5.38 \\
\hline & \pm sem & 3.8 & 0.27 & 0.48 & 0.34 & 0.30 & 0.30 \\
\hline 13 & 50 & 24.9 & 9.02 & 7.78 & 7.89 & 6.42 & 6.42 \\
\hline 14 & 50 & 28.0 & 11.0 & 8.80 & 8.31 & 6.79 & 6.67 \\
\hline 15 & 50 & 26.9 & 8.35 & 7.44 & 6.86 & 5.90 & 5.90 \\
\hline 16 & 50 & 40.1 & 7.89 & 6.58 & 5.51 & & 5.02 \\
\hline 17 & 50 & 38.6 & 8.56 & 7.41 & 6.46 & 5.68 & 5.68 \\
\hline \multirow[t]{3}{*}{18} & 50 & 38.0 & 9.29 & 8.81 & 7.26 & & 7.26 \\
\hline & $\overline{\mathbf{x}}$ & 32.8 & 9.02 & 7.80 & 7.05 & 6.20 & 6.16 \\
\hline & \pm sem & 2.8 & 0.44 & 0.36 & 0.41 & 0.25 & 0.32 \\
\hline 19 & 60 & 38.4 & 10.6 & 8.51 & 7.45 & 7.19 & 7.19 \\
\hline 20 & 60 & 36.9 & 11.8 & 9.10 & 8.12 & & 7.23 \\
\hline 21 & 60 & 32.6 & 10.6 & 10.3 & 8.53 & & 8.53 \\
\hline 22 & 60 & 38.6 & 9.83 & 8.70 & 6.16 & & 6.16 \\
\hline \multirow[t]{3}{*}{23} & 60 & 62.2 & 12.8 & 9.42 & 8.28 & & 8.28 \\
\hline & $\overline{\mathbf{x}}$ & 41.7 & 11.3 & 9.20 & 7.71 & 7.19 & 7.48 \\
\hline & $\pm \mathrm{sem}$ & 5.2 & 0.5 & 0.32 & 0.43 & 0 & 0.43 \\
\hline
\end{tabular}


234

APPENDIX B

DETERMINATION OF OXYGEN UPT AKE 


\section{Determination of Oxygen Untake}

The open circuit technique is a direct method used for measuring aerobic power since it requires the collection and analysis of an individual's expired air. Oxygen uptake expressed as (1. $\left.\mathrm{min}^{-1}\right)$ or $\left(\mathrm{ml} \cdot \mathrm{kg}^{-1} \cdot \mathrm{min}^{-1}\right.$ ) is equal to the difference between the total amount of inspired oxygen and the total amount of expired oxygen:

$$
\dot{V} O_{2}=(\dot{V})\left(F, O_{2}\right)-\left(\dot{V}_{E}\right)\left(F_{E} O_{2}\right)(\text { Equation } 1)
$$

where,

$V_{\mathrm{l}}=$ volume of inspired gas $\left(\mathrm{I} \cdot \mathrm{min}^{-1}\right.$ STPD)

$V_{E}=$ volume of inspired gas $\left(1 \cdot \mathrm{min}^{-1} \mathrm{STPD}\right)$

$\mathrm{F}_{1} \mathrm{O}_{2}=$ fraction of oxygen in inspired gas

$\mathrm{F}_{\mathrm{E}} \mathrm{O}_{2}=$ fraction of oxygen in expired gas

Since nitrogen is physiologically inert, and thus does not take an active part in respiratory gas exchange, the volume of inspired nitrogen $\left(V_{t} N_{2}\right)$ is equal to the volume of expired nitrogen $\left(V_{E} N_{2}\right)$, and therefore $V_{\mathrm{I}}$ can be calculated:

$$
\begin{gathered}
\left(\dot{V}_{l}\right)\left(F_{t} N_{2}\right)=\left(\dot{V}_{E}\right)\left(F_{E} N_{2}\right), \\
\dot{V}_{1}=\frac{\left(\dot{V}_{E}\right)\left(F_{E} N_{2}\right)}{F_{r} N_{2}}(\text { Equation } 2)
\end{gathered}
$$

where,

$V_{\varepsilon}$ is measured via the expired gas sample

$F_{E} N_{2}=$ fraction of nitrogen in expired gas

$\mathrm{F}_{\mathrm{I}} \mathrm{N}_{2}=$ fraction of nitrogen in inspired gas

Since the inspired air is composed of $\mathrm{N}_{2}(79.04 \%), \mathrm{O}_{2}(20.93 \%)$ and $\mathrm{CO}_{2}(.03 \%), \mathrm{F}_{\mathrm{I}} \mathrm{N}_{2}, \mathrm{~F}_{\mathrm{I}} \mathrm{O}_{2}$, and $\mathrm{F}_{1} \mathrm{CO}_{2}$ are assumed as constants:

$\mathrm{F}_{1} \mathrm{~N}_{2}=.7093$

$\mathrm{F}_{1} \mathrm{O}_{2}=.2093$

$\mathrm{F}_{1} \mathrm{CO}_{2}=.0003$

therefore:

$$
F_{E} N_{2}=1-\left(F_{E} O_{2}+F_{E} \mathrm{CO}_{2}\right)(\text { Equation } 3)
$$

where,

$\mathrm{F}_{\mathrm{E}} \mathrm{O}_{2}$ and $\mathrm{F}_{\mathrm{E}} \mathrm{CO}_{2}$ are measured via the rapid response electronic analyzers.

Returning to Equation $1, \mathrm{VO}_{2}\left(1 \cdot \mathrm{min}^{-1}\right)$ can now be determined. 
APPENDIX C

DETERMINATION OF a-v̄DO ${ }_{2}$ FROM THE ACETYLENE REBREATHE TECHNIQUE 


\section{Determination of $\mathrm{a}-\mathrm{yDO} \mathrm{O}_{2}$ from the Acetylene Rebreathe Technique}

The acetylene rebreathe technique is an effective method used for measuring cardiac output at rest and at all levels of exercise. The procedure involves rebreathing a mixture of nitrogen, oxygen and acetylene, from a latex bag. Once the gases equilibrate in the lung-bag system, an initial sample of each separate gas is analyzed. Approximately eight to ten seconds later, a final sample of each gas is analyzed. The volume of expired air is typically smaller than the volume of inspired air because a little less carbon dioxide is normally returned than oxygen is consumed. Since a change in volume of the lungbag system would influence the concentrations of expired oxygen and acetylene, and since nitrogen does not take an active part in respiratory gas exchange, a nitrogen correction factor is applied to account for any change in volume over time:

$$
V_{1}=\left(V_{2}\right)\left(\frac{\left(N_{2}\right)_{2}}{\left(N_{2}\right)_{1}}\right)(\text { EquationI })
$$

where,

$\mathrm{V}_{1}$ = initial volume in the lung-bag system

$\mathrm{V}_{2}=$ final volume in the lung-bag system

$\left(\mathrm{N}_{2}\right)_{1}=$ initial concentration of nitrogen

$\left(\mathrm{N}_{2}\right)_{2}=$ final concentration of nitrogen

The volume of acetylene absorbed by the lung during the sampling period is:

$$
\left.\left.\left(V_{2}\right)\left(\frac{\left(N_{2}\right)_{2}}{\left(N_{2}\right)_{1}}\right)\left(C_{2} H_{2}\right)_{1}-\left(V_{2}\right) C_{2} H_{2}\right)_{2} \text { (Equation } 2\right)
$$

where,

$\left(\mathrm{C}_{2} \mathrm{H}_{2}\right)_{1}=$ initial concentration of acetylene

$\left(\mathrm{C}_{2} \mathrm{H}_{2}\right)_{2}=$ final concentration of acetylene

The initial concentration of acetylene corrected for the change in volume of the lung-bag system alters Equation2:

$$
\left.\left(V_{2}\right)\left(C_{2} H_{2}\right)_{c}-\left(C_{2} H_{2}\right)_{2}\right](\text { Equation } 3)
$$

where.

$\left(\mathrm{C}_{2} \mathrm{H}_{2}\right)_{c}=$ initial concentration of acetylene corrected for the change in volume of the lung-bag system

Similarly, the volume of oxygen absorbed by the lung during the sampling period is:

$$
\left.\left(V_{2}\right)\left(O_{2}\right)_{c}-\left(O_{2}\right)_{2}\right](\text { Equation } 4)
$$

where,

$\left(\mathrm{O}_{2}\right)_{\mathrm{c}}=$ initial concentration of oxygen corrected for the change in volume of the lung-bag system 
The average concentration of acetylene during the sampling period $\left(\mathrm{x}_{2} \mathrm{H}_{2}\right)$ is:

$$
\text { IC } C_{2} H_{2}=\frac{\left(C_{2} H_{2}\right)_{c}\left(C_{2} H_{2}\right)_{2}}{2}(\text { Equation })
$$

At body temperature and at a barometric pressure $\left(\mathrm{P}_{\mathrm{b}}\right)$ of $760 \mathrm{mmHg}$, one litre of blood will dissolve $740 \mathrm{ml}$ of acetylene. Thus, the volume of acetylene absorbed per litre of blood during the sampling period is:

$$
\left(\frac{(740)\left(f C_{2} H_{2}\right)}{100}\right)\left(\frac{P_{2}-47}{760}\right) \text { (Equation6) }
$$

where,

47 = assumed water vapour pressure in the lungs (in $\mathrm{mmHg}$ )

The volume of blood perfusing the lungs during the sampling period is:

$$
\frac{\left(V_{2}\right)\left(C_{2} H_{2}\right)_{d}\left(C_{2} H_{2}\right)_{2}(760)(100)}{(740)\left(\bar{X} C_{2} H_{2}\right)\left(P_{b}-47\right)}(\text { Equation } 7)
$$

The volume of oxygen absorbed divided by the volume of blood passing through the lungs during the sampling period is equal to the arterial-mixed venous oxygen difference $\left(\mathrm{ml} \mathrm{O}_{2} \cdot 1\right.$ blood $\left.^{-1}\right)$ :

$$
a-\bar{v} D O_{2}=\frac{\left(O_{2}\right)_{d i j g}\left(\bar{x} C_{2} H_{2}\right)\left(P_{b}-47\right)(0.00974)}{\left(C_{2} H_{2}\right)_{d i d}}(\text { Equation } 8)
$$

where,

$0.00974=$ tissue solubility coefficient of acetylene gas

Via the Fick Equation, cardiac output and stroke volume can now be calculated:

$$
\dot{Q}=\frac{\dot{V} O_{2}}{a-\bar{v} D O_{2}}(\text { Equation9) }
$$

and,

$$
S V=\frac{\dot{Q}}{H R}(\text { Equation10 })
$$


APPENDIX D

DETERMINATION OF PLASMA VOLUME AND BLOOD VOLUME 


\section{Determination of Plasma Volume and Blood Volume}

The use of Evan's Blue Dye is an effective method used for measuring plasma volume (ml) since it is firmly binds to plasma albumin and leaves the vascular system slowly (6-10\% per hour). Before blood volume $(\mathrm{ml})$ or $\left(\mathrm{ml} \cdot \mathrm{kg}^{-1}\right)$ can be calculated, plasma volume must be determined:

$$
P V=\left(\frac{20 k}{D_{2}}\right) \cdot F(\text { Equation } 1)
$$

where,

$D_{2}=$ optical density of the "Dyed Plasma" sample at 610nm.

$F=$ correction factor based on the amount of dye injected per subject $(0.75=15 \mathrm{mg}$ or $3 \mathrm{ml}$ of dye injected)

$k=$ constant determined for each dye lot injected, and is calculated as:

$$
k=\frac{D_{1}}{C}(\text { Equation } 2)
$$

where,

$D_{1}=$ optical density of the standard containing the "Dye-Free" plasma sample at $610 \mathrm{~nm}$.

$\mathrm{C}=$ concentration of the dye lot following dilution (constant $0.004 \mathrm{mg} / \mathrm{ml}$ of plasma)

The amount of dye injected depends upon the subject's body mass (eg. $15 \mathrm{mg}$ of dye is required for subjects who weigh 100-180 lbs.)

Blood volume can be calculated from the following formula:

$$
B V=\left(\frac{P V}{100-H c t}\right) \cdot 100(\text { Equation } 3)
$$




\section{Determination of Body Density and Percent Body Fat}

Jackson \& Pollock (134) developed generalized equations, by testing 403 men 18 to 61 years (1978) and 331 women 18 to 55 years (1980) and correlating anthropometric measures to hydrostatic weighing. They seperated men and women in their statistical analyses, and recognized a curvilinear relationship between skinfold measurements and body density. The body density of fernales is calculated as follows:

\section{BodyDensity $=1.0970-0.00046971\left(\sum\right.$ of 7 skinfolds $)+0.00000056\left(\sum \text { of } 7 \text { skinfolds }\right)^{2}-0.00012828$ (age $)$}

Body density values are converted to percent body fat values by the Brozek et al. (16) equation:

$$
\text { BrozekEquation:\%Fat }=\left(\frac{4.57}{\text { BodyDensity }}-4.142\right) \cdot 100
$$

The normative data utilized for this technique recommend 22 to $28 \%$ for women for "adult fitness" (134). 
APPENDIX F

INFORMED CONSENT 


\section{CARDIOVASCULAR CHANGES WUTH AGE IN ENDURANCE TRAINGD FEMALES}

\section{INFORMED CONSENT DOCUMENT}

As a participant in a research study, it is important that you are well informed about the procedures used in the study and the potential risks involved. This document outlines the specific procedures involved in the study of Dr. N. Gledhill and Miss Colleen Wiebe (Department of Exercise and Health Sciences, 736-5794). This study has been approved by York University's Human Participants ethics committee (736-5055).

\section{Direct Assessment of Aerobic Power:}

You will exercise at progressive stages to maximum on the cycle ergometer while breathing through a mouthpiece into a collecting tank for 4 minutes at each workload. Heart rate will be monitored using electrodes attached to the chest and by a wireless heart monitor. Heart sounds will be measured using a heart sound microphone attached to the chest by adjustable straps. The pulse of blood through the ear will be monitored by a photocell attached to the left ear.

2. Gas Mixture Rebreathe:

Towards the end of each stage of the progressive cycle ergometer test, you will exhale completely and then inhale a gas mixture consisting of oxygen, nitrogen and a small percentage of acetylene. You will then breathe this gas mixture back and forth into a bag for approximately 8 - 12 breaths two times per workload.

\section{Blood Measurements:}

a) Determination of Blood Volume: You will receive an injection of dye through a vein in the forearm. After a 10 minute period, a $5 \mathrm{ml}$ sample of blood will be withdrawn from a vein in the lower arm.

b) Venous blood sample: Each test day you will have venous blood drawn from an indwelling catheter in a vein in the arm or back of the hand. Resting blood samples will be collected prior to each test.

c) NOTE: It will be necessary for you to refrain from consuming alcohol and caffeine and from engaging in exercise in the 24 hours prior to each test day. While undergoing intense exercise, you may experience episodes of nausea, dizziness or other discomforts. Although blood samples are taken and infusions given by an individual qualified in the technique, there is a slight chance of infection and/or bruising. Every 
precaution will be taken to minimize or prevent any ill-effects. Dr. Peter Rowan, a physician who has been working with the laboratory for a number of years, will provide medical assistance as required. You will be required to come in on 3 separate occasions during this study. This will require a total of approximately 6 hours of participation.

I, the undersigned, have read and understood the above statements. I understand that I may ask questions at any time regarding this document or the procedures involved in this study, and that I may withdraw from the testing at any time I desire. I also understand that signing this form does not constitute release or waiver of my legal rights concerning the study or the researchers involved.

I agree to participate in the study outlined in this document.

Name: Signature:

(please print)

Date:

Witness: Signature:

(please print) 

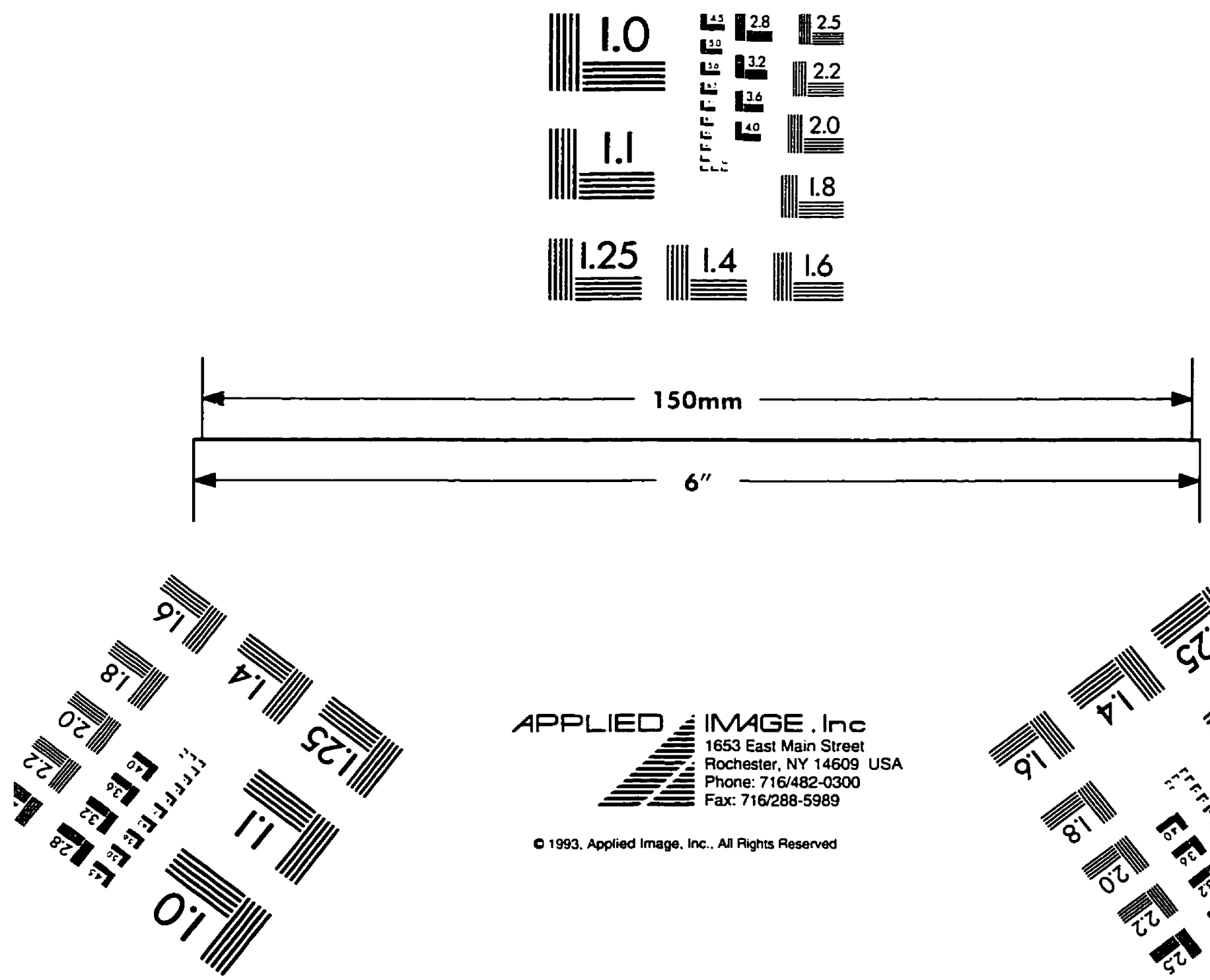

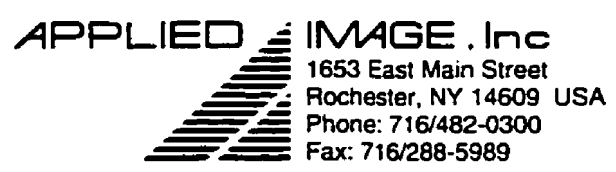

O 1993. Applied Image. Inc.. All Rights Reserved

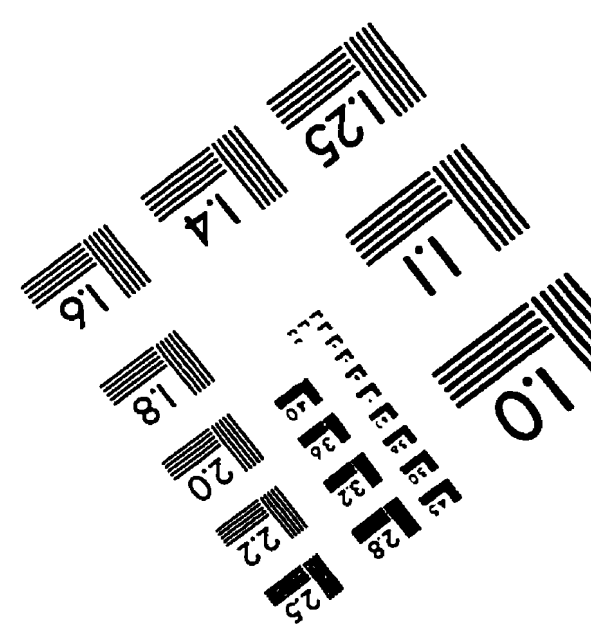

